\title{
THE WORLD BEYOND BLACK HOLES
}

Light contains the key to open the doors to Heaven. Unfortunately, the same key fits on the doors to Hell

Author:

Country:

Website:

Email:

\section{$\underline{\text { Wim Vegt }}$}

The Netherlands

https://wimvegt.topworld.center

j.w.vegt@topacademy.center 


\section{Books from Wim Vegt in the series: "The POWER OF LIGHT":}

11) 150 YEARS PHYSICS based on the WRONG EQUATION. (EBook) ISBN: 9789402192896 . Paperback ISBN: 9789402192735.

10) The Nikola Tesla Way of Energy Transport. (E-book) ISBN:

9789402191349. Paperback ISBN: 9789402190984.

9) The Rise of ELF Electromagnetic Attack Weapons and the Necessity of the Development of Corresponding ELF Defense Systems. (E-book) ISBN: 9789402189544 . Paperback ISBN: 9789402189117

8) Unified 4-Dimensional Hyperspace Equilibrium. (E-book) ISBN: 9789402181036. Paperback ISBN: 9789402180985

7) Beyond Superstrings. (E-book) ISBN: 9789402179668 . Paperback ISBN: 9789402179637

6) The Hidden World Behind Superstrings. (E-book) ISBN: 9789402180053

5) Light is the Bridge between God, Relativity and Quantum Physics (E-book) ISBN: 9789402178975

4) The Particle-Wave-Mass Unification. A New Theory in Quantum Physics.

(E-book) ISBN: 9789402178647. Paperback ISBN: 9789402178586

3) The Tri-Unity in Religion and in Science. (Paperback) ISBN: 9789402178531

2) The Power of the LIGHT rules over the SHADOWS of the DARKNESS (Paperback) ISBN: 9789402178326

1) The Bridge of Light (E-book) ISBN: 9789402177947. Paperback ISBN: 9789402177763 


\section{PREFACE}

This is a book about the world beyond Black Holes and has been divided into three parts.

The first part, Level 1, describes the theory about Black Holes at a fundamental level which is understandable for everyone with a few years of basic Physics education. The reader is familiar with fundamental equations like Newton's second Law of motion $(\mathrm{F}=\mathrm{m}$ a) and Einsteins's famous relationship between mass and energy $(E=m$ $\left.c^{2}\right)$.

The second part, Level 2, describes the theory about Black Holes at a university level which is understandable for everyone with a Physics university education. The reader is familiar with fundamental mathematics like tensor calculus.

The third part, Level 3, describes the complete New Theory and offers new insights in the mathematical approach for Black Holes and the relationship with quantum physics, electric charge, magnetic spin and infra-red shift. 


\section{Classical Theory New Theory Newton \\ NEWTON in 3 dimensions \\ Newton \\ NEWTON in 4 dimensions}

$$
\begin{array}{r}
\overline{\mathrm{F}}=\mathrm{m} \overline{\mathrm{a}} \\
\left(\begin{array}{l}
\mathrm{x}_{3} \\
\mathrm{x}_{2} \\
\mathrm{x}_{1}
\end{array}\right) \rightarrow\left(\begin{array}{l}
\mathrm{F}_{\mathrm{z}} \\
\mathrm{F}_{\mathrm{y}} \\
\mathrm{F}_{\mathrm{x}}
\end{array}\right)=\mathrm{m}\left(\begin{array}{l}
\mathrm{a}_{\mathrm{z}} \\
\mathrm{a}_{\mathrm{y}} \\
\mathrm{a}_{\mathrm{x}}
\end{array}\right)
\end{array}
$$

$$
\begin{array}{r}
\overline{\mathrm{F}}^{4}=\mathrm{m} \frac{-4}{\mathrm{a}^{4}} \\
\left(\begin{array}{l}
\mathrm{x}_{4} \\
\mathrm{x}_{3} \\
\mathrm{x}_{2} \\
\mathrm{x}_{1}
\end{array}\right) \rightarrow\left(\begin{array}{l}
\mathrm{F}_{\mathrm{t}} \\
\mathrm{F}_{\mathrm{z}} \\
\mathrm{F}_{\mathrm{y}} \\
\mathrm{F}_{\mathrm{x}}
\end{array}\right)=\mathrm{m}\left(\begin{array}{l}
\mathrm{a}_{\mathrm{t}} \\
\mathrm{a}_{\mathrm{z}} \\
\mathrm{a}_{\mathrm{y}} \\
\mathrm{a}_{\mathrm{x}}
\end{array}\right)
\end{array}
$$

Maxwell (James Clerk Maxwell)

$$
\begin{aligned}
& \nabla \cdot \overline{\mathrm{E}}=\frac{\rho}{\varepsilon} \\
& \nabla \times \overline{\mathrm{E}}=-\mu \frac{\partial \overline{\mathrm{H}}}{\partial \mathrm{t}} \\
& \nabla \cdot \overline{\mathrm{H}}=0 \\
& \nabla \times \overline{\mathrm{H}}=\varepsilon \frac{\partial \overline{\mathrm{E}}}{\partial \mathrm{t}}
\end{aligned}
$$

Dirac/ Schrôdinger (Newton $4^{\text {th }}$ Dimension)

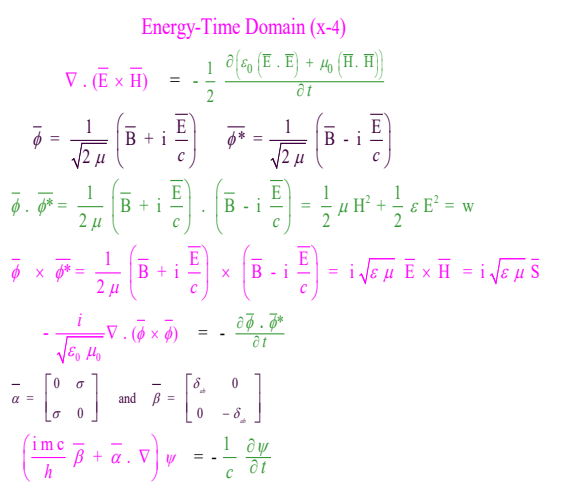

Quantum Gravity (Newton $4^{\text {th }}$ Dimension)

Energy-Time Domain (x-4)

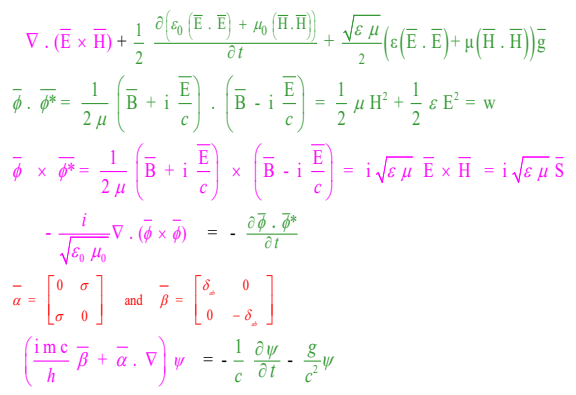

\section{Maxwell (Newton in 3 Dimensions)}

$$
\begin{aligned}
& \text { NEWTON: } \rightarrow \overline{\mathrm{F}}_{\text {TOOTLL }}=\mathrm{m} \overline{\mathrm{a}}[\mathrm{N}] \rightarrow \overline{\mathrm{f}}_{\text {TOTIL }}=\rho \overline{\mathrm{a}}\left[\mathrm{N} / \mathrm{m}^{3}\right] \\
& -\rho \overline{\mathrm{a}}+\quad \overline{\mathrm{f}}_{\text {rotul }} \quad=\overline{0}\left[\mathrm{~N} / \mathrm{m}^{3}\right] \\
& -\rho \overline{\mathrm{a}}+\overline{\mathrm{f}}_{\text {HeCtric }}+\overline{\mathrm{f}}_{\text {Mäentic }}=\overline{0}\left[\mathrm{~N} / \mathrm{m}^{3}\right]
\end{aligned}
$$

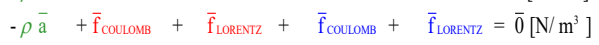

$$
\begin{aligned}
& -\frac{1}{c^{2}} \frac{\partial(\mathrm{E} \times \mathrm{H})}{\partial t}+\varepsilon_{0} \overline{\mathrm{E}}(\nabla \cdot \overline{\mathrm{E}})-\varepsilon_{0} \overline{\mathrm{E}} \times(\nabla \times \overline{\mathrm{E}})+\mu_{0} \overline{\mathrm{H}}(\nabla \cdot \overline{\mathrm{H}})-\mu_{0} \overline{\mathrm{H}} \times(\nabla \times \overline{\mathrm{H}})=0\left[\mathrm{~N} / \mathrm{m}^{3}\right]
\end{aligned}
$$

Dirac/ Schrődinger (Newton $4^{\text {th }}$ Dimension)

$$
\begin{aligned}
& \text { Energy-Time Domain }(\mathrm{x}-4) \\
& \text { B-6 B-7 } \\
& \nabla \cdot(\overline{\mathrm{E}} \times \overline{\mathrm{H}})=-\frac{1}{2} \frac{\partial\left(\varepsilon_{0}(\overline{\mathrm{E}} \cdot \overline{\mathrm{E}})+\mu_{0}(\overline{\mathrm{H}} \cdot \overline{\mathrm{H}})\right)}{\partial t} \\
& \text { 3-Dimensional Space Domain } \\
& \text { B-1 B-2 B-3 } \\
& \left(\begin{array}{c}
\mathrm{x}_{3} \\
\mathrm{x}_{2} \\
\mathrm{x}_{1}
\end{array}\right) \quad \begin{array}{cc}
-\frac{1}{2} \frac{\partial(\overline{\mathrm{E}} \times \overline{\mathrm{H}})}{\partial t}+\varepsilon_{0} \overline{\mathrm{E}}(\nabla \cdot \overline{\mathrm{E}})-\varepsilon_{0} \overline{\mathrm{E}} \times(\nabla \times \overline{\mathrm{E}})+ \\
\quad \mathrm{B}-4 \\
+\mu_{0} \overline{\mathrm{H}}(\nabla \cdot \overline{\mathrm{H}})-\mu_{0} \overline{\mathrm{H}} \times(\nabla \times \overline{\mathrm{H}})=\overline{0}
\end{array}
\end{aligned}
$$

Quantum Gravity (Newton $4^{\text {th }}$ Dimension)

$$
\text { Energy-Time Domain }
$$

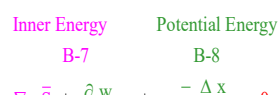

$\left(x_{4}\right)$

$$
\nabla \cdot \overline{\mathrm{S}}+\frac{\partial \mathrm{W}}{\partial t}+\rho \overline{\mathrm{g}} \frac{\Delta \mathrm{x}}{\Delta \mathrm{t}}=0
$$

3-Dimensional Space Domain

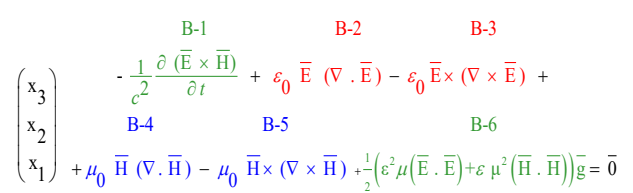


1.0 Black Holes Level 1

1.1 Introduction $\underline{8}$

1.2 Inertia, the Interaction between Confined $\underline{14}$ Electromagnetic Energy and Gravity

1.3 Inertia and the change in the relative radiation $\underline{16}$ pressure

$1.4 \quad$ Inertia and the relative change of the radiation $\quad \underline{20}$ pressures of confined water jets

1.5 Inertia and the relative change of the radiation 23 pressures of confined laser beams

1.6 The anisotropic effects of inertia 27

1.7 The equilibrium boundary between gravity and $\underline{29}$ the radiation pressure

1.8 The event Horizon

1.9 The Transversal Black Hole

1.10 The Fundamental Equation for the $\underline{38}$ Electromagnetic Field

1.10.1 The Term of Inertia (Term B-1) $\underline{39}$

1.10.2 The Electric Force Density (Coulomb's Law $\underline{40}$ Term B-2)

1.10.3 The Magnetic Force Density (Lorentz Force $4 \underline{41}$ Term B-5)

1.10.4 The Fundamental Equation for the $\underline{43}$ Electromagnetic field

1.11 The Fundamental Equation for the Black Hole, 44 describing Gravitational-Electromagnetic Interaction

1.12 Longitudinal Black Holes $\underline{46}$

1.13 Longitudinal Black Hole with an $\underline{48}$ electromagnetic mass of $10^{-4}[\mathrm{~kg}]$ and a radius $=$ $\left.2 \times 10^{-35}[\mathrm{~m}]\right)$ :

1.14 Longitudinal Black Hole with an electromagnetic mass of $10^{40}[\mathrm{~kg}]$, a radius $=$ $1.5 \times 10^{9}[\mathrm{~m}]$ at a frequency of $0.2[\mathrm{~Hz}]$ :

1.15 Quantum Gravity described by Newton's second $\underline{54}$ law of motion in the 4th dimension (time domain)

1.16 Data Availability 


\section{Black Holes (level 1)}

\subsection{Introduction}

To understand the physics of Black Holes, it is important to understand the first law in Physics which controls our entire universe. This is the law of "Perfect Equilibrium". Within the entire universe there is always a prefect equilibrium between all the physical forces like gravity, forces of inertia, radiation pressure and Electro-Magnetic Interaction forces at any time, in any direction and at any space coordinate. This is the fundamental law in physics on which also the existence of a Black Hole has been grounded.

This new theory will explain the forces within a beam of light interacting with gravity while the beam of light propagates within the gravitational field generated by a black hole.

When we look at modern Physics, we can only be impressed by an enormous amount of knowledge and a complete New World of technical applications. We now live in the century of the impressive victory of the new science and the new technology over the oldfashioned world and the old-fashioned way of thinking.

Great shifts in the way of thinking and the technological achievements are mostly characterized by an important scientific publication in a century that changes everything in that century. We can recognize the century of Isaac Newton who triggered in 1687 the seismic shift in thinking with his famous publication "Philisophiae Naturalis Principia Mathematica" (Mathematical Principles of Natural Philosophy).

We recognize the century of James Clerk Maxwell who triggered in 1865 the large shift in thinking with his famous publication "A Dynamical Theory of the Electromagnetic Field".

We recognize the century of Albert Einstein who triggered in 1905 the large changings in thinking with his famous theory of Special Relativity represented in his publication "On the Electrodynamics of Moving Bodies". Manifesting a "New Theory" and a "New Way of 
Thinking" with important contributions of Hendrik Lorentz, Henri Poincaré and Hermann Minkowski.

It is recognizable that with the sudden change in thinking in a new period, a new kind of mutual common sense and a general agreement by many scientists of the the new theory and the new way of thinking arises. The New Theory will be protected by common sense and mutual agreement. This new way of thinking settles down in the scientific society and become immovable. Other options disappear and simply do not exist anymore.

Different from the alpha and the gamma sciences, the beta sciences are being developed by a kind of a LEGO system. Building blocks built one after another and built on top of each other. Like we build with the LEGO system houses and castles using the same LEGO building block over and over again, we build in the beta sciences grand theories, using basic the same basic equations over and over again. A large shift in the beta sciences happens when a new mathematical building block has been developed. Like the equations of Newton or the equations of Maxwell or the Schrödinger and the Dirac equations. These fundamental equations form the mathematical LEGO system of our modern scientific world.

Because these mathematical building blocks are being used over and over and again in numerous applications over a period of of more than 100 years, a general scientific common sense rises around these mathematical building blocks. This scientific common sense protects these mathematical building blocks like a high wall around a medieval town.

A fundamental problem rises, when one of these building blocks is not correct or turns out not to be correct under certain conditions. Like the famous Law of Newton for the relationship between acceleration (a), mass (m) and force (F): " $\mathrm{F}=\mathrm{m}$ a" turns out not to be valid at velocities near the speed of light because at these velocities the mass is changing. It took a long time before Albert Einstein's theory of general relativity had been accepted, because his theory of general relativity was in contradiction with the famous well-known mathematical building blocks which had already been used and being protected for hundreds 
of years. But nowadays Einstein's famous theory of general relativity has been accepted world-wide.

This book describes a comparable conflict in the modern beta sciences and brings the well-known and generally accepted Modern Physics of the last 150 years in question. Because when a fundamental mathematical building block, which has been introduced 150 years ago and has been used to develop the Modern Physics during the last 150 years, turns out to be wrong (or not complete), a fundamental problems rises in Modern Physics, developed during the last 150 years.

This situation happens in relation with the well-known Maxwell Equations, presented 150 years ago in the famous publication: " $A$ Dynamical Theory of the Electromagnetic Field" in 1865. which has been used as a fundamental mathematical building block in many modern physical theories.

In Maxwell's time there were no optical LASERS (Light Amplification by Stimulated Emission of Radiation) and the outcome of his theory was in his time completely in correspondence with what could be measured at that time. The value for the speed of light, calculated from the Maxwell Equations, corresponded almost exactly with the value for the speed of light measured in 1862 by Léon Foucault by a system of rotating mirrors and measured in 1877 by Albert Michelson (300.140 $[\mathrm{km} / \mathrm{s}])$.

But nowadays there arises several problems with Maxwell's theory for the electromagnetic field. Since the existence of the LASERS it became clear that the speed of light is not always the same in every direction. When a beam of light, generated by a LASER, propagates with the well-known speed of light "c $=299.792[\mathrm{~km} / \mathrm{s}]$ " in the $\mathrm{z}$-direction, the speed of light equals zero in the $\mathrm{x}$-direction and the $\mathrm{y}$-direction (in a orthogonal $\mathrm{x}, \mathrm{y}, \mathrm{z}$ frame).

This new phenomenon cannot be explained by Maxwell's Theory. In Maxwell's Theory the speed of light has to be exactly the same in every direction. This is clearly not the fact for a LASER beam. And also for the projection of a slide on a screen, it is clearly that the speed of light within the plane of the screen equals zero. Because the slide we observe does not move. While the projection beam itself moves towards the 
screen with the speed of light "c", the beam clearly remains focused and does not move within the plane, perpendicular to the direction of propagation.

Another effect which cannot be explained by Maxwell's Theory about electromagnetism has been demonstrated within the IBM research group. A new, until 1995 unknown, experiment has been conducted by: O. Gunawan, Y Virgus and K. Fai Tai to demonstrate a subtle hidden feature in electromagnetism - a previously unknown field confinement effect that they named the "camelback effect" (Ref. 1) in a system of two lines of transverse dipoles.

In electromagnetism, the elementary source of electric field and magnetic field can be respectively modeled as a point charge - a hypothetical charge located at a single point in space - and a dipole, a pair of equal and oppositely charged or magnetized poles separated by a distance. Imagine we line up two rows of magnetic dipoles and we try to measure the strength of the magnetic field along the center axis. The magnetic field is certainly stronger at the center and diminishes away from it. However, if the length of the dipole line exceeds certain critical length, a surprising effect occurs: the field gets slightly stronger near the edges and produces a field confinement profile that looks like a camel's back - hence the name of the effect. The IBM team has reported this discovery with detailed experimental and theoretical studies in two recent publications and patents.

This surprising discovery is exciting for a few reasons. First, it represents a new elementary one-dimensional confinement potential in physics, joining the list of well-known potentials such as Coulomb, parabolic, and square well. Second, this effect becomes the key feature that enables this system to serve as a new class of natural magnetic trap (Ref. 2) called parallel dipole line (PDL) trap with many possible exciting applications. This camelback effect and the related PDL magnetic trap can be realized using special cylindrical magnets whose poles are on the curved side and a graphite rod as the trapped object.

This new, until 1995 unknown, effect can only be explained by electromagnetic interaction, described in the New Theory (Ref. 3). 
A recent experiment ${ }^{(5)}$ in 2019 at the Yale University in New Haven C.T. USA published in Nature with the title: "To catch and and reverse a quantum jump mid-flight" conflicts a fundamental aspect of the Copenhagen Interpretation related to "Fundamental Uncertainty" (Probability) represented within the "Standard Model" in Quantum Physics (Ref. 4).

In quantum physics, measurements can fundamentally yield discrete and random results. Emblematic of this feature is Bohr's 1913 proposal of quantum jumps between two discrete energy levels of an atom. Experimentally, quantum jumps were first observed in an atomic ion driven by a weak deterministic force while under strong continuous energy measurement

The times at which the discontinuous jump transitions occur are reputed to be fundamentally unpredictable. Despite the nondeterministic character of quantum physics, is it possible to know if a quantum jump is about to occur? Here we answer this question affirmatively: we experimentally demonstrate that the jump from the ground state to an excited state of a superconducting artificial threelevel atom can be tracked as it follows a predictable 'flight', by monitoring the population of an auxiliary energy level coupled to the ground state. The experimental results demonstrate that the evolution of each completed jump is continuous, coherent and deterministic.

The only explanation for this deterministic effect has been described within the new theory in equation $(5.7$, Page 152) which originated form the deterministic electromagnetic field.

There is no other conclusion than the conclusion that the Maxwell Equations are "wrong" or at least "not complete". The right equation(s) have to describe both possibilities. The possibility that the light moves in every direction with the exactly the same speed of light "c" like the light being emitted by the sun. And the possibility that the light moves only in one direction and equals zero in the directions perpendicular to the plane of propagation like the propagation of a LASER beam.

A second conclusion can only be that fundamental quantum mechanical relations like the Schrödinger wave equation and the relativistic Dirac equation both originate from a deterministic field like an 
electromagnetic field which has been demonstrated in the new theory in equation $(5.7$, Page 152$)$.

To find these new equation(s) we observe that the Maxwell equations are not in unification with Newton's theory of equilibrium of forces. The Maxwell Equations are not in unification with Newtons $3^{\text {rd }}$ law "action $=-$ reaction". Maxwell has not included the force densities with an electromagnetic field at all. To find this new equation, we have to introduce the force densities within an electromagnetic field. 


\subsection{Inertia, the Interaction between Confined Electromagnetic Energy and Gravity}

Black Holes are a challenge in modern Physics. The greatest challenge for the "Reader" of this book will be to "Let it Go", to "Let it Pass Away", to "Forget what you have learned". To become simple and humble again and most of all: be "Be Not Impressed" of what Modern Physics has achieved the last 300 years. Because in Modern Physics we did not get too far. We got far in technology and we got far in applications of technology, but in the understanding of Physics we did not get much further than Isaac Newton 300 years ago. Since Isaac Newton, not much has changed in our physical concept of the Universe. The knowledge of Isaac Newton already contains the "Philosopher's Stone", the "Key of Wisdom". The key to open the gates to the hidden world of knowledge.

The "Philosopher's Stone" refers to the hidden and deep understanding of. "Light". As it was written in the holy books. Like it has been written in the Jewish "Torah-Pentateuch-Bereishit-Genesis 1:1-5" and in the Christian Bible in Genesis 1:1-5.

In the beginning God created the heavens and the earth. ${ }^{2}$ Now the earth was formless and empty, darkness was over the surface of the deep, and the Spirit of God was hovering over the waters. ${ }^{3}$ And God said, "Let there be light," and there was light. ${ }^{4}$ God saw that the light was good, and he separated the light from the darkness. ${ }^{5}$ God called the light "day," and the darkness he called "night." And there was evening, and there was morning - the first day.

Described in a different version in the Muslim Religion in "The Verse of Light" in the $35^{\text {th }}$ verse of the $24^{\text {th }}$ Sura of the Quran.

Our first knowledge starts with the knowledge of Light. And the "Black Hole" is like the "Anti-God". The great "Absorber of the Holy Light". The unholiest place in the universe from which no Holy Light can escape. An "Eternal Prison for Light". A kind of Hell in the Spiritual world becomes a place of Eternal Darkness in this world in our universe. 
How would Isaac Newton have looked at the phenomenon of a "Black Hole"?

In Newton's time it was impossible to speak about a phenomenon like a Black Hole. A place in the Universe in which all the light disappears. Because in Newton's time Light was symbolic for "God". And a Black Hole eating and destroying Light would be like the Devil eating and destroying God. Newton immediately would been accused of evil witchcraft and worshipping the Devil. And Newton would have been burnt alive on the stakes because in his time it was still usual to burn witches and other heretics on the medieval stakes.

This fact makes it very interesting, because maybe Newton knew about the possibility of Black Holes in the Universe. Because it is wellknown that Newton kept many of his secret writings hidden for the scientific world. And the fact that Newtons famous equation of motion turns out to be a theoretical and mathematical foundation for the existence of Black Holes in the universe.

Newton published his famous second law of motion in 1705 in "Philosophiae Naturalis Principia Mathematica" which will turn out to be the foundation for the possible existence of Black Holes in the Universe, when Newton's second law of motion will be applied to a beam of light within a gravitational field. 


\subsection{Inertia and the change in the relative radiation pressure}

To understand this mathematical approach in physics, it is necessary to understand the concept of "inertia". The property of inertia for "matter" as well the property of inertia for "light".

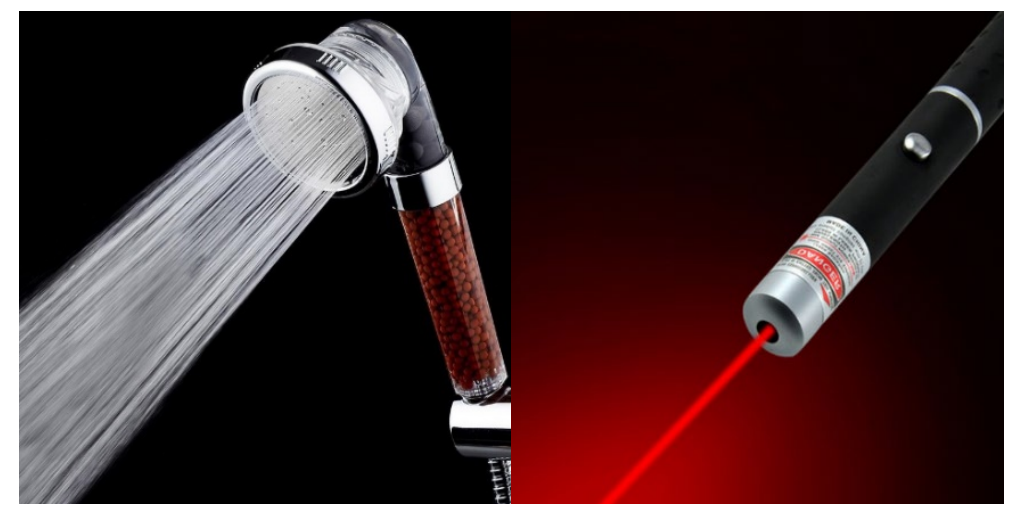

Figure 1 The comparison of a waterjet emitted by a shower head and a laser beam emitted by a laser.

To understand the property of "inertia" for a beam light, we start with an imaginary model of a beam of light, presented by a jet of water leaving a shower head.

Imagine you hold your right hand in the water jet, emitted by a shower head. Then you will feel the radiation pressure of the water jet, comparable with the radiation pressure of a beam of light emitted by a laser. 


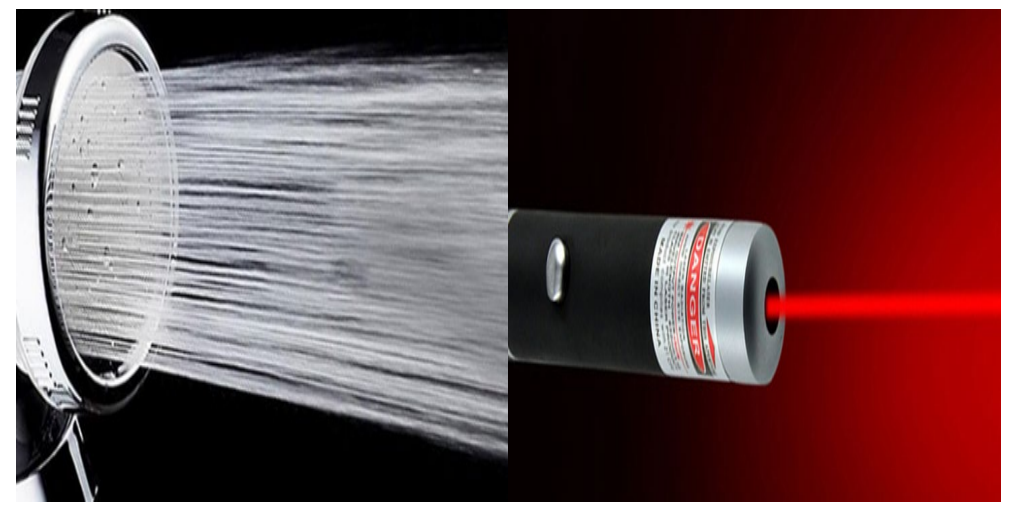

Figure 2 The shower head emits a waterjet towards to the right and the laser emits a laser beam towards to the right.

When you move your right hand towards to the left (in the opposite direction of the water jet), you will feel that the radiation pressure of the water jet increases. When you move your hand towards to the right (in the direction of the water jet) you will feel that the radiation pressure of the water jet decreases.

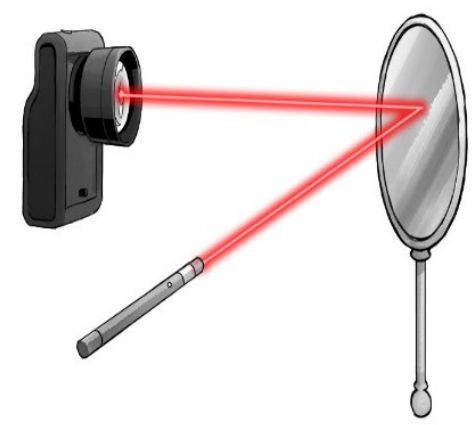

Figure 3. A laser beam has been reflected by the mirror (placed at the right from the laser) and acts with an electromagnetic radiation pressure on the mirror directed towards to the right.

In a comparable way the radiation pressure of a laser beam emitted by a laser acting on the mirror, will increase when we move the mirror towards to the left (towards the laser, in the opposite direction of propagation of the laser beam). The radiation pressure on the mirror will decrease when we move the mirror towards to the right (away from the laser, in the direction of propagation of the laser beam). 
Of course, the radiation pressure of the beam of laser light will be much smaller compared to the radiation pressure of the water jet. But with very sensitive equipment the radiation pressure of the beam of laser light can be measured. As an example, the radiation pressure on earth of the light emitted by the sun, equals about $10\left[\mu \mathrm{N} / \mathrm{m}^{2}\right]$. Which equals a total radiation pressure of the sunlight acting on the surface of the whole earth of about $1.2510^{9}[\mathrm{~N}]$.

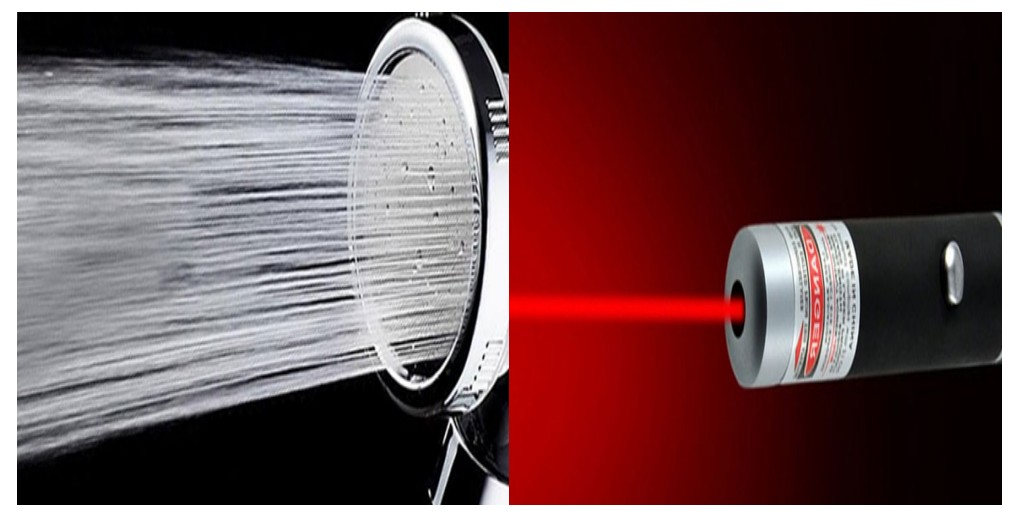

Figure 4. The shower head emits a waterjet towards to the left and the laser emits a laser beam towards to the left. 
Now we choose a shower head and a laser emitting towards the left. When you move your left hand towards to the left (in the direction of the water jet), you will feel that the radiation pressure of the water jet decreases. When you move your left hand towards to the right (in the opposite direction of the water jet) you will feel that the radiation pressure of the water jet increases.

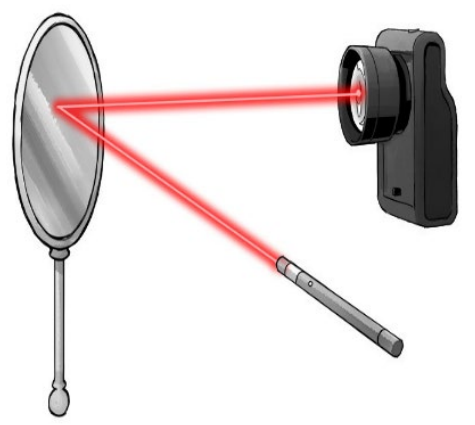

Figure 5. A laser beam has been reflected by the mirror (placed at the left from the laser) and acts with an electromagnetic radiation pressure on the mirror directed towards to the left.

In a comparable way the radiation pressure of a laser beam emitted by a laser acting on the mirror, will decrease when we move the mirror towards to the left (away from the laser, in the direction of propagation of the laser beam). The radiation pressure on the mirror will increase when we move the mirror towards to the right (in the opposite direction of the laser beam). 
1.4 Inertia and the relative change of the radiation pressures of confined water jets

To demonstrate the property of inertia, we place the right hand in the water jet, emitted towards to the right by the first shower head. The the left hand has been placed in the water jet, emitted towards to the left by the second shower head.

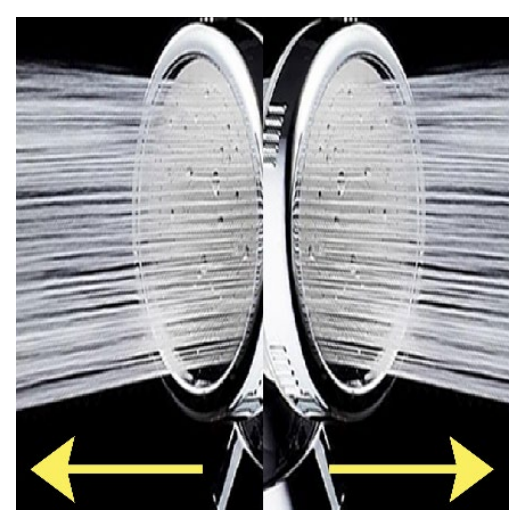

Figure 6. Two shower heads, pointing in opposite directions, emit two waterjets pointing in opposite directions. The left hand has been placed in the waterjet emitted towards to the left and the right hand has been placed in the waterjet emitted towards to the right.

The radiation pressure of the water jet acting on the left hand equals the radiation pressure acting on the right hand but has been directed oppositely. Both radiation pressures neutralize each-other and the resulting force acting on the system of both hands equals zero. 


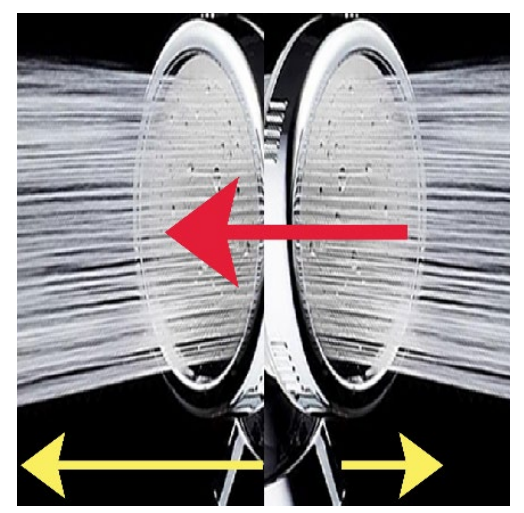

Figure 7. The left hand has been placed in the waterjet emitted towards to the left and the right hand has been placed in the waterjet emitted towards to the right. The resulting force has been oriented towards to the Left.

To demonstrate the property of inertia of the confined water jets between both hands, we move both hands simultaneously towards to the right. The radiation pressure of the waterjet acting on the left hand will increase. The radiation pressure of the waterjet acting on the right hand will decrease. The total resulting radiation pressures of both waterjets will not neutralize each-other anymore. The total resulting radiation pressure of both waterjets will be directed towards to the left, opposite in the direction of moving. In figure 7 this has been represented by the red arrow pointing towards to the left. We experience the effect of inertia. Both waterjets resist the starting movement of our both hands towards to the right, when we consider both hands together as one mechanical system.

We replace both hands by one (plastic) box, placed over both waterjets. When we move the box towards to the right, we will experience the (extra) inertia of the box, caused by the resulting force of both waterjets directed towards to the left. This is the resulting inertia force, represented by the red arrow in figure 7 . Moving the box towards to the right results in an inertia force in the opposite direction represented by the red arrow in figure 7. 


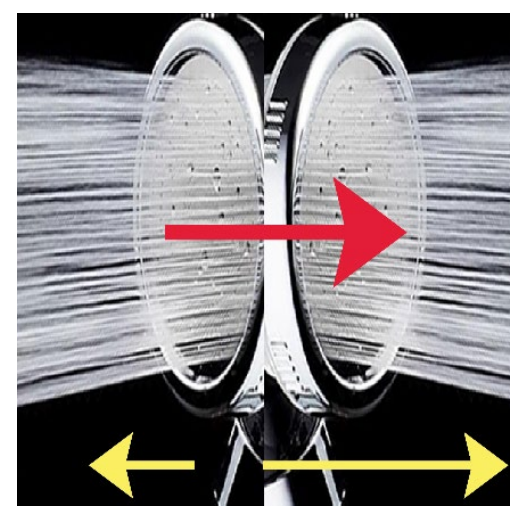

Figure 8. The left hand has been placed in the waterjet emitted towards to the left and the right hand has been placed in the waterjet emitted towards to the right. The resulting force has been oriented towards to the Right.

To demonstrate the property of inertia of the confined water jets between both hands, we move both hands simultaneously towards to the left. The radiation pressure of the waterjet acting on the left hand will decrease. The radiation pressure of the waterjet acting on the right hand will decrease. The total resulting radiation pressures of both waterjets will not neutralize each-other anymore. The total resulting radiation pressure of both waterjets will be directed towards to the left, opposite in the direction of moving. In figure 7 this has been represented by the red arrow pointing towards to the left. We experience the effect of inertia. Both waterjets resist the starting movement of our both hands towards to the left, when we consider both hands together as one mechanical system.

We replace both hands by one (plastic) box, placed over both waterjets. When we move the box towards to the right, we will experience the (extra) inertia of the box, caused by the resulting force of both waterjets directed towards to the left. This is the resulting inertia force, represented by the red arrow in figure 8 . Moving the box towards to the left results in an inertia force in the opposite direction represented by the red arrow in figure 8. 
1.5 Inertia and the relative change of the radiation pressures of confined laser beams

To demonstrate the property of inertia of the electromagnetic radiation (laser beam), confined between both mirrors, we place the right mirror in the laser beam, emitted towards to the right by the first laser. The left mirror has been placed in the laser beam, emitted towards to the left by the second laser.

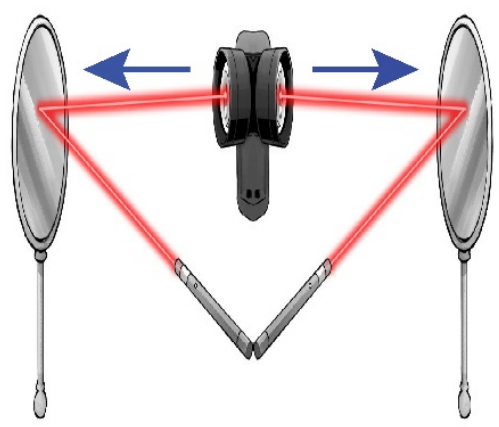

Figure 9. Two lasers, pointing in opposite directions, emit two laser beams pointing in opposite directions. The left mirror has been placed in the laser beam emitted towards to the left and the right mirror been placed in the laser beam towards to the right

The radiation pressure of the laser beam acting on the left mirror equals the radiation pressure acting on the right mirror but has been directed oppositely. Both radiation pressures neutralize each other, and the resulting force acting on the system of both mirrors equals zero. 


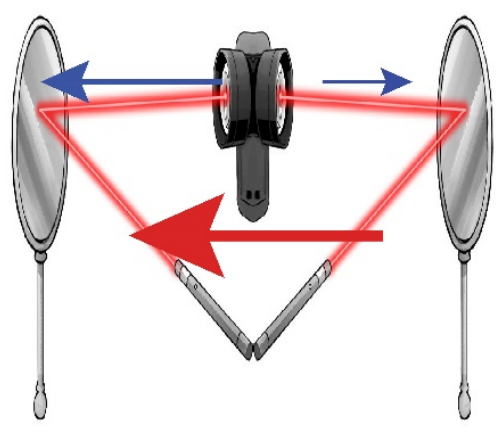

Figure 10. The left mirror has been placed in the laser beam emitted towards to the left and the right mirror been placed in the laser beam towards to the right. The resulting force has been directed towards tot the Left.

To demonstrate the property of inertia of the confined laser beams between between both mirrors, we move both mirrors simultaneously towards to the right. The radiation pressure of the laser beam acting on the left mirror will increase. The radiation pressure of the laser beam acting on the right mirror will decrease. The total resulting radiation pressures of both laser beams will not neutralize each-other anymore. The total resulting radiation pressure of both laser beams will be directed towards to the left, opposite in the direction of moving. In figure 10 this has been represented by the red arrow pointing towards to the left. We experience the effect of inertia. Both laser beams resist the starting movement of our both mirrors, when we consider both mirrors together as one mechanical system.

We replace both mirrors by a box with at the inside $100 \%$ reflecting mirrors. The confined electromagnetic radiation (laser beam) between both mirrors will act on both mirrors with an oppositely directed radiation pressure.

When we start to move the box towards to the right, the resulting total radiation pressure will be oriented towards tot the left. This is the inertia force of the confined electromagnetic radiation. Albert Einstein demonstrated the proportionality between mass (inertia) and energy (intensity of electromagnetic radiation of the confined laser beam) by his famous equation: $\mathrm{E}=\mathrm{m} \mathrm{c}^{2}$. 


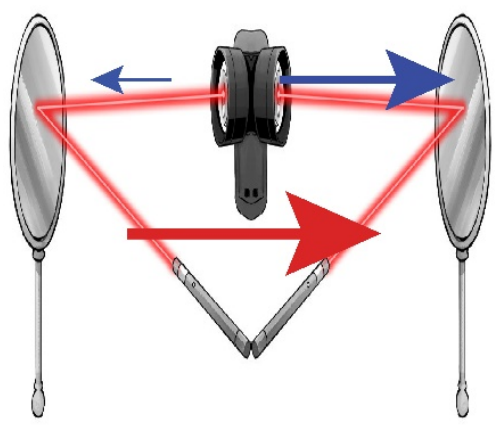

Figure 11. The left mirror has been placed in the laser beam emitted towards to the left and the right mirror been placed in the laser beam towards to the right. The resulting force has been directed towards tot the Right.

To demonstrate the property of inertia of the confined laser beams between between both mirrors, we move both mirrors simultaneously towards to the left. The radiation pressure of the laser beam acting on the left mirror will decrease. The radiation pressure of the laser beam acting on the right mirror will increase. The total resulting radiation pressures of both laser beams will not neutralize each-other anymore. The total resulting radiation pressure of both laser beams will be directed towards to the right, opposite in the direction of moving. In figure 11 this has been represented by the red arrow pointing towards to the right. We experience the effect of inertia. Both laser beams resist the starting movement of our both mirrors, when we consider both mirrors together as one mechanical system.

We replace both mirrors by a box with at the inside $100 \%$ reflecting mirrors. The confined electromagnetic radiation (laser beam) between both mirrors will act on both mirrors with an oppositely directed radiation pressure.

When we start to move the box towards to the left, the resulting total radiation pressure will be oriented towards tot the right. This is the inertia force of the confined electromagnetic radiation. Albert Einstein demonstrated the proportionality between mass (inertia) and energy (intensity of electromagnetic radiation of the confined laser beam) by his famous equation: $\mathrm{E}=\mathrm{m} \mathrm{c}^{2}$. 
We replace in a "thought experiment" the box with at the inside $100 \%$ reflecting mirrors by a sphere with a $100 \%$ reflecting inside. The hollow sphere contains confined electromagnetic radiation.

Proportional to the total confined electromagnetic energy inside the sphere, the sphere will demonstrate the property of "inertia" in any direction according Newton's second law of motion: $F=\mathrm{m}$. a.

Now we replace the hollow sphere with confined electromagnetic radiation by a "Gravitational-Electromagnetic Confinement" of electromagnetic radiation. In a comparable way the electromagnetic confinement will demonstrate the property of "inertia", represented by Newton's second law of motion: $F=m$. a. 


\subsection{The anisotropic effects of inertia}

A laser beam demonstrates an "anisotropic" effect of inertia. Which means that the effect of inertia is not the same in any direction, like the inertia of confined electromagnetic radiation within a hollow sphere. Only "Confined Electromagnetic Energy" demonstrates the effect of inertia according Newton's second law of motion.

In the directions, perpendicular to the direction of propagation of the laser beam, the electromagnetic energy of the Laser beam has been confined. In these directions, perpendicular to the direction of propagation, the laser beam acts according Newton's second law of motion $\mathrm{F}=\mathrm{m}$. a. The mass density of the laser beam has been determined by Einstein's $\mathrm{E}=\mathrm{m} \mathrm{c}^{2}$. When we divide the left and right terms of Einstein's equation by the Volume, we find a relationship between the energy density " $w$ " and the mass density " $\rho$ ". Einstein's equation can be written as: $\mathrm{w}=\rho \mathrm{c}^{2}$.

This equation is the fundamental equation for the representation of a "Transversal Black Hole" in which a beam of light follows a circular orbit around a Black Hole due to the Gravity force acting on the mass density " $\rho$ " of the beam of light. Comparable with a satellite moving in a (circular) orbit around the earth because of the gravity force of the earth acting on the mass of the satellite.

In the direction of propagation, the electromagnetic energy has not been confined. In the direction of the propagation of a laser beam does exist a perfect equilibrium between the forward directed radiation pressure and the inertia (mass) of the electromagnetic energy density " $\rho$ ". For this reason, it is impossible to accelerate or to decelerate the propagation speed of a laser beam according Newton's second law of motion.

This is comparable with the speed of sound for a sound wave, which will not be influenced by the existence of a gravitational field. A beam of light moving towards a Black Hole or away from a Black Hole will keep the same speed of light. But the intensity of the beam of Light will increase when the beam of light moves towards a Black Hole and the intensity will decrease when a beam of light moves away from a Black 
Hole. This type of confinement has been called a "Longitudinal Black Hole" in which the confined light always propagates in the direction of the gravitational field of the Black Hole. 


\subsection{The equilibrium boundary between gravity and the radiation pressure}

Light is the phenomenon of electromagnetic radiation, propagating with the speed of light, because of the outside directed radiation pressure. The speed of light has been determined by the perfect equilibrium between the radiation pressure and the inertia of the electromagnetic mass density.

For spherical waves, the radiation pressure of the electromagnetic energy is equal in any direction and the speed of light is equal in any direction.

For a Laser beam, the radiation pressure has been counterbalanced by electro-magnetic interaction in the directions perpendicular to the direction of propagation. These are the directions in which the electromagnetic field components, the electric field and the magnetic field, have been oriented. Only in these directions the "electromagnetic interaction" can exist. In the direction perpendicular to these directions, electromagnetic interaction cannot exist. And the radiation pressure will be counterbalanced by the inertia of the electromagnetic energy. This results in the propagation speed of the laser beam perpendicular to the directions of the electric field and the magnetic field with the wellknown speed of light.

When a Laser beam enters a gravitational field, the perfect equilibrium between the radiation pressure and the inertia of the electromagnetic energy will be disturbed by the interaction of the gravitational field on the electromagnetic mass density of the Laser beam.

Within a gravitational field the radiation pressure will not change, the electromagnetic interaction will not change, but the total force acting on the electromagnetic mass density of the Laser beam will change.

This results in two effects.

1) A straight propagation path of a Laser beam will turn into a circular orbit of the Laser beam when the gravitational field has been oriented perpendicular to the direction of propagation. 
2) The intensity of the Laser beam will decrease or increase when the gravitational field has been oriented in the direction of propagation. But the speed of propagation (speed of light) will not change. Because the radiation pressure represents an alternating force density (with the same frequency as the frequency of the Laser beam) acting on the inertia of the electromagnetic energy. And the gravitational field acts with a stationary force density on the same inertia of the electromagnetic energy. 


\subsection{The event Horizon}

The "event horizon" is the boundary defining the region of space around a black hole from which nothing (not even light) can escape. The "event horizon" has been created by the equilibrium between the gravitational forces and the forces of electromagnetic interaction and the radiation pressure. At the boundary of a Black Hole does exist the prefect equilibrium. There are two types of Black Holes.

The first type represents an equilibrium in which the Black Hole has been represented by a hollow sphere. Only within the extreme thin shell of the hollow sphere does exist a perfect equilibrium. Inside the hollow sphere any kind of light (electromagnetic radiation) has been pressed with an extreme high radiation pressure towards to the "Shell of Perfect Equilibrium" (Event Horizon) because the radiation pressure inside the hollow sphere is always larger than the confining gravitational force. Outside the hollow sphere, any kind of light (electromagnetic radiation) has been attracted towards the "Shell of Perfect Equilibrium" (Event Horizon) because outside the hollow sphere the confining gravitational force is always larger than the radiation pressure. This type has been called "Transversal Black Holes". Because like a planet moves within an orbit around a star, any beam of light (electromagnetic radiation) propagates within the Shell of Perfect Equilibrium" (Event Horizon) in an orbit around the Black Hole.

The second type represents an Equilibrium everywhere around the black Hole. At a special distance from the center of the Black Hole, the radiation density increases suddenly very sharp. This has been called the Boundary (Event Horizon) around the Black Hole. This type has been called "Longitudinal Black Holes". Because the light (electromagnetic radiation) moves towards the Black Holes or away from the Black Hole in the same direction as the direction of the Gravitational Field generated by the Black Hole. 


\subsection{The Transversal Black Hole}

We consider a beam of light passing a strong gravitational field, generated by a Black Hole. According the first term in (1) the beam of light will follow a circular orbit around the Black Hole. The required Equilibrium will exist at the radius where the centrifugal electromagnetic inertia forces will be equal and opposite directed to the centripetal oriented gravitational forces on the electromagnetic mass. Figure 12 represents the orbit (colored red) of a LASER beam around a uniform intense gravitational field (Black Hole)

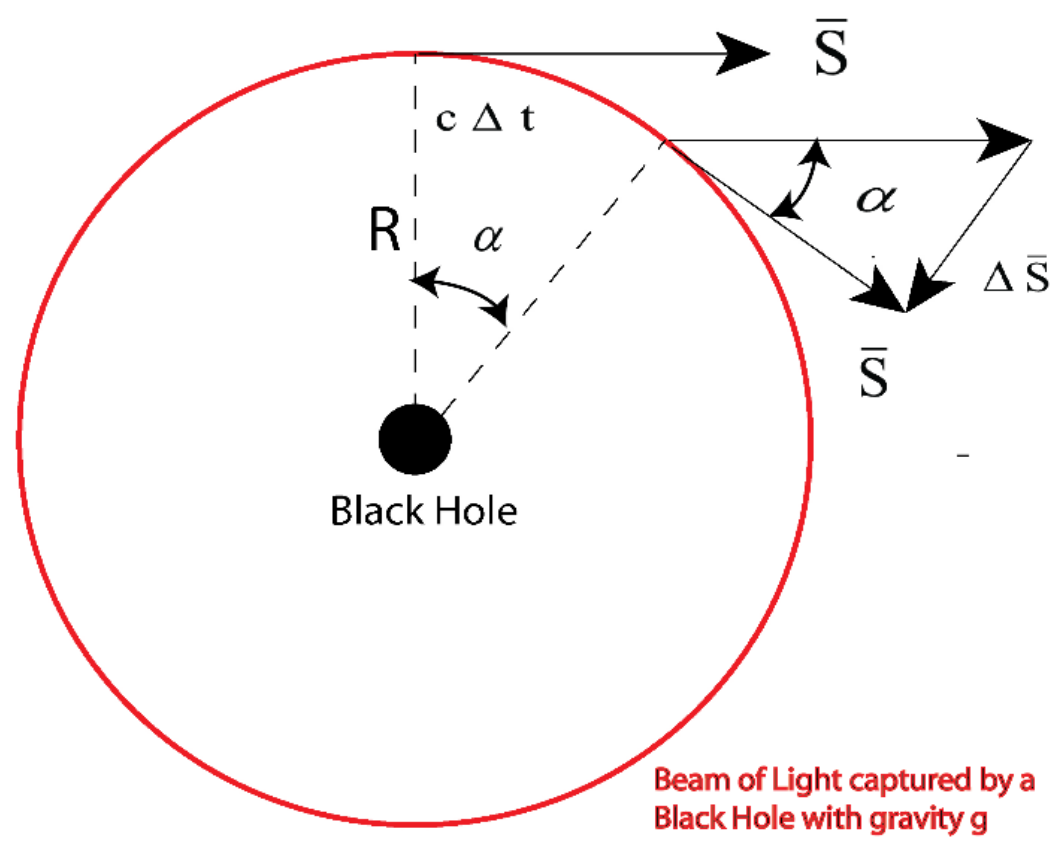

Fig. 12. A LASER beam around a Black Hole captured in a circular orbit around the Black Hole in a Transversal Modus by the Gravitational interaction of the Black Hole with the mass (inertia) of the Laser Beam Light.

In general, Newtons second law of motion has been presented as:

$$
\mathrm{F}=\mathrm{m} \mathrm{a}
$$

In which "a" represents the acceleration which equals the difference of 
the velocity $\Delta \mathrm{v}$ divided by the time interval $\Delta \mathrm{t}$.

$$
\mathrm{a}=\frac{\Delta \mathrm{v}}{\Delta \mathrm{t}}
$$

The momentum $\mathrm{p}$ of a mechanical mass equals:

$$
\mathrm{p}=\mathrm{m} \mathrm{v}
$$

Then Newton's second law of motion can be presented as:

$$
\text { (mechanical mass) }
$$

$$
\mathrm{F}_{\text {INERTIA }}=\mathrm{m} \mathrm{a}=\mathrm{m} \frac{\Delta \mathrm{v}}{\Delta \mathrm{t}}=\frac{\Delta(\mathrm{m} \mathrm{v})}{\Delta \mathrm{t}}=\frac{\Delta(\mathrm{p})}{\Delta \mathrm{t}}
$$

Like a mechanical mass expresses the property of inertia, also a beam of light expressed the property of inertia. When the sun shines on the earth, the radiation of the sun presses on the earth with thousands of Newton.

Like a mechanical mass, also a beam of light has momentum. The momentum of a beam of light has been expressed by the Poynting vector $\mathrm{S}$ and equals the mechanical momentum vector $\mathrm{p}$ multiplied by the square of the speed of light $\mathrm{c}$ divided by the Volume.

$$
\begin{gathered}
\text { (mechanical mass) (beam of light) } \\
\mathrm{F}_{\text {INERTIA }}=\mathrm{m} \mathrm{a}=\mathrm{m} \frac{\Delta \mathrm{v}}{\Delta \mathrm{t}}=\frac{\Delta(\mathrm{m} \mathrm{v})}{\Delta \mathrm{t}}=\frac{\Delta(\mathrm{p})}{\Delta \mathrm{t}}=\frac{\mathrm{V}}{\mathrm{c}^{2}} \frac{\Delta(\mathrm{S})}{\Delta \mathrm{t}}
\end{gathered}
$$

The inertia force density "f" equals the inertia force "F" divided by the Volume "V".

$$
\text { (mechanical mass) (beam of light) }
$$

$$
f_{\text {INERTIA }}=\left(\frac{\mathrm{m}}{\mathrm{V}}\right) \mathrm{a}=\rho \frac{\Delta \mathrm{v}}{\Delta \mathrm{t}}=\frac{1}{\mathrm{c}^{2}} \frac{\Delta(\mathrm{S})}{\Delta \mathrm{t}}
$$

The well-known equation of Einstein equals: 
$\mathrm{W}=\mathrm{m} \mathrm{c}^{2}$

$\mathrm{W}=\frac{\mathrm{W}}{\mathrm{V}}=\frac{\mathrm{m}}{\mathrm{V}} \mathrm{c}^{2}=\rho \mathrm{c}^{2}$

In which "w" represents the electromagnetic energy density and equals:

$\mathrm{w}=\frac{1}{2} \varepsilon \mathrm{E}^{2}+\frac{1}{2} \mu \mathrm{H}^{2}$

For electromagnetic radiation the electromagnetic impedance $\mathrm{Z}_{0}$ equals:

$\mathrm{Z}_{0}=\frac{\mathrm{E}}{\mathrm{H}}=\sqrt{\frac{\mu}{\varepsilon}}$

$\mathrm{H}=\mathrm{E} \sqrt{\frac{\varepsilon}{\mu}}$

$\mathrm{w}=\frac{1}{2} \varepsilon \mathrm{E}^{2}+\frac{1}{2} \mu \mathrm{H}^{2}=\frac{1}{2} \varepsilon \mathrm{E}^{2}+\frac{1}{2} \varepsilon \mathrm{E}^{2}=\varepsilon \mathrm{E}^{2}$

Substituting equation (9) in (7) results in:

$$
\begin{aligned}
& \mathrm{w}=\rho \mathrm{c}^{2}=\varepsilon \mathrm{E}^{2} \\
& \rho=\frac{\varepsilon}{\mathrm{c}^{2}} \mathrm{E}^{2}
\end{aligned}
$$

Because the beam of light has been confined in the radial direction, it demonstrates in the radial direction the property of inertia (electromagnetic mass) and interacts with a gravitational field according Newton's second law of motion. The whole Universe is in a perfect Equilibrium. Also at the "Event Horizon" of a Black Hole does exist a perfect equilibrium between the confining gravitational force of the Black Hole and the radial directed inertia force density of the confined electromagnetic radiation (Laser Beam confined by a Black Hole at the "Event Horizon").

To determine the "Event Horizon" of the Black Hole (Radius of the circular orbit of the Laser Beam), we have to find the perfect equilibrium between the inertia force densities of the electromagnetic energy 
densities of the Laser Beam and the confining gravitational force acting on the electromagnetic energy densities of the Laser Beam.

$$
\begin{aligned}
& \mathrm{f}_{\text {GRAVITY }}=\mathrm{f}_{\text {INERTIA }} \\
& \rho \mathrm{g}=\frac{1}{c^{2}} \frac{\Delta(\mathrm{S})}{\Delta \mathrm{t}}
\end{aligned}
$$

From Figure (12) follows the relationship between de changing in the Poynting vector $\Delta \mathrm{S}$ and the time interval $\Delta \mathrm{t}$ :

$$
\begin{aligned}
& \operatorname{Tan}(\alpha)=\frac{\mathrm{c} \Delta \mathrm{t}}{\mathrm{R}}=\frac{\Delta \mathrm{S}}{\mathrm{S}} \\
& \frac{\Delta \mathrm{S}}{\Delta \mathrm{t}}=\frac{\mathrm{c} \mathrm{S}}{\mathrm{R}} \\
& \rho \mathrm{g}=\frac{1}{\mathrm{c}^{2}} \frac{\Delta(\mathrm{S})}{\Delta \mathrm{t}}=\frac{1}{\mathrm{c}^{2}} \frac{\mathrm{c} \mathrm{S}}{\mathrm{R}}=\frac{1}{\mathrm{c}} \frac{\mathrm{S}}{\mathrm{R}}
\end{aligned}
$$

The Poynting vector $\overline{\mathrm{S}}$ represents the total energy transport of the electromagnetic radiation per unit surface per unit time $\left[\mathrm{J} / \mathrm{m}^{2} \mathrm{~s}\right]$. Which can be written as the cross product of the Electric Field intensity $\overline{\mathrm{E}}$ and the magnetic Field intensity $\overline{\mathrm{H}}$.

$$
\begin{aligned}
& \overline{\mathrm{S}}=\overline{\mathrm{E}} \times \overline{\mathrm{H}} \\
& \mathrm{S}=\mathrm{E} \mathrm{H} \operatorname{Sin}\left(90^{\circ}\right)=\mathrm{E} \mathrm{H} \\
& S=\mathrm{E}^{2} \sqrt{\frac{\varepsilon}{\mu}}
\end{aligned}
$$

Substituting equation (10) and (13) in (12) results in an equation for the Event Horizon at radius " $R$ " of a Transversal Black Hole. 


$$
\begin{aligned}
& \rho \mathrm{g}=\frac{1}{\mathrm{c}} \frac{\mathrm{S}}{\mathrm{R}} \\
& \mathrm{R}=\frac{\mathrm{S}}{\rho \mathrm{c} g} \\
& R=\frac{\mathrm{E}^{2} \sqrt{\frac{\varepsilon}{\mu}}}{\rho \mathrm{c} g}=\frac{\mathrm{E}^{2} \sqrt{\frac{\varepsilon}{\mu}}}{\frac{\varepsilon}{\mathrm{c}} \mathrm{E}^{2} \mathrm{~g}} \\
& \mathrm{R}=\frac{\mathrm{c}^{2}}{\mathrm{~g}} \approx \frac{910^{16}}{g}[\mathrm{~m}]
\end{aligned}
$$

Equation (14) represents the perfect equilibrium between the inertia force densities of the electromagnetic mass $\frac{1}{c^{2}} \frac{\Delta \bar{S}}{\Delta t}$ and the centripetal oriented gravitational force density $\frac{\mathrm{w}}{\mathrm{c}^{2}} \overline{\mathrm{g}}$ acting on the electromagnetic mass. The perfect equilibrium direction $[9,10,12,13]$ where the inertia forces due to the circular orbit of the beam of light are in a perfect balance with the attractive gravitational forces, exists at one defined radius " $R$ " of the beam of light (LASER beam), independent of the intensity of the beam of light and independent of the frequency of the beam of light. Only the acceleration " $\mathrm{g}$ " of the gravitational field determines the radius of equilibrium "R"

$$
\mathrm{R} \approx \frac{910^{16}}{g}
$$

In which " $\mathrm{R}$ " is the radius of the beam of light and "g" the acceleration of the gravitational field of the "Black Hole".

The $\mathrm{x}$-y plane is oriented perpendicular on the z-direction. The speed of light towards the positive z-direction equals the speed of light (the constant "c $=300.000 \mathrm{~km} / \mathrm{s} ")$. But the speed of light in the $\mathrm{x}-\mathrm{y}$ plane has to be exactly zero $[9,14,15]$. Else the diameter of the laser beam would become larger and larger during the propagation along the positive zdirection. This is only possible because the Electromagnetic confining forces B-2, B-3, B-4 and B-5 compensate exactly the outward oriented 
radiation pressure towards the $\mathrm{x}$-direction and the $\mathrm{y}$-direction.

The Electric Radiation Pressure has been compensated by the Coulomb Force Densities within the Laser Beam 


\subsection{The Fundamental Equation for the Electromagnetic Field}

In a fundamental way, Newton's second law of motion describes the required electromagnetic equation for the GravitationalElectromagnetic Interaction for a Longitudinal Black Hole.

Because Maxwell's 4 equations are not part of one whole uniform understanding of the universe like the fundamental equation of Newton's second law of motion represents.

Newton's second law of motion has been based on a profound understanding of the universe which is based on the fundamental principle of Harmony and Equilibrium.

To describe the interaction between light and gravity it is important to define the fundamental equation for the electromagnetic field based on this fundamental principle of Harmony and Equilibrium formulated by Newton in 1687 published in his famous work: "Philosophiae Naturalis Principia Mathematica (Mathematical Principles of Natural Philosophy".

To realize this, Newton's second law of motion will be the ground on which the New Theory will have been built. The fundamental Electromagnetic force density equation has been based integral on Newton's second law of motion and has been divided into 5 separate terms (B-1 - B-5).

The first term B-1 represents the inertia of the mass density of light (Electromagnetic Radiation). The terms B-2 and B-3 represent the electric force densities within the Electromagnetic Radiation (Beam of Light) and the terms B-4 and B-5 represent the magnetic force densities within the Electromagnetic Radiation (Beam of Light). 


\subsubsection{The Term of Inertia (Term B-1)}

The right and the left term of Newton's law of motion in equation (16) has been divided by the Volume " $V$ " to find an equation for the force density " $f$ " related to the mass density " $\rho$ ".

$$
\begin{aligned}
\mathrm{F} & =\mathrm{m} \mathrm{a} \\
\left(\frac{\mathrm{F}}{\mathrm{V}}\right) & =\left(\frac{\mathrm{m}}{\mathrm{V}}\right) \mathrm{a} \\
\mathrm{f} & =\rho \mathrm{a}
\end{aligned}
$$

Electromagnetic Inertia Force Density (Term B-1)

$$
\begin{aligned}
& \mathrm{F}_{\text {INERTIA }}=-\mathrm{m} \mathrm{a}=-\mathrm{m} \frac{\Delta \mathrm{v}}{\Delta \mathrm{t}}=-\frac{\Delta(\mathrm{m} \mathrm{v})}{\Delta \mathrm{t}}=-\frac{\Delta \mathrm{p}}{\Delta \mathrm{t}}=-\left(\frac{V}{\mathrm{c}^{2}}\right) \frac{\Delta \mathrm{S}}{\Delta \mathrm{t}} \\
& \frac{\mathrm{F}_{\text {INERTIA }}}{\mathrm{V}}=-\frac{\mathrm{m}}{V} \mathrm{a}=-\frac{\mathrm{m}}{V} \frac{\Delta \mathrm{v}}{\Delta \mathrm{t}}=-\frac{1}{V} \frac{\Delta \mathrm{p}}{\Delta \mathrm{t}}=-\left(\frac{1}{\mathrm{c}^{2}}\right) \frac{\Delta \mathrm{S}}{\Delta \mathrm{t}} \\
& \mathrm{f}_{\text {INERTIA }}=-\rho \mathrm{a}=-\left(\frac{1}{\mathrm{c}^{2}}\right) \frac{\Delta \mathrm{S}}{\Delta \mathrm{t}}\left[\mathrm{N} / \mathrm{m}^{3}\right]
\end{aligned}
$$

The Poynting vector $\overline{\mathrm{S}}$ represents the total energy transport of the electromagnetic radiation per unit surface per unit time $\left[\mathrm{J} / \mathrm{m}^{2} \mathrm{~s}\right]$. Which can be written as the cross product of the Electric Field intensity $\overline{\mathrm{E}}$ and the magnetic Field intensity $\overline{\mathrm{H}}$.

$$
\begin{aligned}
& \mathrm{f}_{\text {INERTIA }}=-\rho \mathrm{a}=-\left(\frac{1}{\mathrm{c}^{2}}\right) \frac{\Delta \mathrm{S}}{\Delta \mathrm{t}}=-\left(\frac{1}{\mathrm{c}^{2}}\right) \frac{\Delta(\overline{\mathrm{E}} \times \overline{\mathrm{H}})}{\Delta \mathrm{t}}\left[\mathrm{N} / \mathrm{m}^{3}\right] \\
& \mathrm{f}_{\text {INERTIA }}=-\left(\frac{1}{\mathrm{c}^{2}}\right) \frac{\partial(\overline{\mathrm{E}} \times \overline{\mathrm{H}})}{\partial \mathrm{t}}\left[\mathrm{N} / \mathrm{m}^{3}\right]
\end{aligned}
$$




\subsubsection{The Electric Force Density (Coulomb's Law Term B-2)}

An example of the Coulomb Force is the Electric Force F $F_{\text {Coulomb acting }}$ on an electric charge $Q$ placed in an electric field $E$. The equation for the Coulomb Force equals:

$\overline{\mathrm{F}}_{\text {Coulomb }}=\overline{\mathrm{E}} \mathrm{Q} \quad[\mathrm{N}]$

Dividing both terms by the Volume V:

$\frac{\overline{\mathrm{F}}_{\text {сойомв }}}{\mathrm{V}}=\overline{\mathrm{E}} \frac{\mathrm{Q}}{\mathrm{V}}\left[\mathrm{N} / \mathrm{m}^{3}\right]$

Results in the Electric force density:

$\overline{\mathrm{f}}_{\text {COUlomb }}=\overline{\mathrm{E}} \rho_{\mathrm{E}}=\overline{\mathrm{E}}(\nabla . \mathrm{D})=\varepsilon \overline{\mathrm{E}}(\nabla . \mathrm{E})\left[\mathrm{N} / \mathrm{m}^{3}\right]$ 


\subsubsection{The Magnetic Force Density (Lorentz Force Term B-5)}

An example of the Lorentz Force is the Magnetic Force F Lorentz $_{\text {acting }}$ on an electric charge $\mathrm{Q}$ moving with a velocity $\mathrm{v}$ within a magnetic field with magnetic field intensity $\mathrm{B}$ (magnetic induction). The equation for the Lorentz Force equals:

$\overline{\mathrm{F}}_{\text {LORENTZ }}=\mathrm{Q} \overline{\mathrm{v}} \times \overline{\mathrm{B}} \quad[\mathrm{N}]$

Dividing both terms by the Volume V:

$\frac{\overline{\mathrm{F}}_{\text {Lorentz }}}{\mathrm{V}}=-\overline{\mathrm{B}} \times \frac{\mathrm{Q} \overline{\mathrm{V}}}{\mathrm{V}}=-\overline{\mathrm{B}} \times \overline{\mathrm{j}}=-\mu \overline{\mathrm{H}} \times \overline{\mathrm{j}}\left[\mathrm{N} / \mathrm{m}^{3}\right]$

Results in the magnetic force density:

$$
\overline{\mathrm{f}}_{\text {LORENTZ }}=-\overline{\mathrm{B}} \times \frac{\mathrm{Q} \overline{\mathrm{V}}}{\mathrm{V}}=-\overline{\mathrm{B}} \times \overline{\mathrm{j}}=-\mu \overline{\mathrm{H}} \times \overline{\mathrm{j}}\left[\mathrm{N} / \mathrm{m}^{3}\right]
$$

In which $q$ is the electric charge, $v$ the velocity of the electric charge, $B$ the magnetic induction and $\mathrm{j}$ the electric current density.

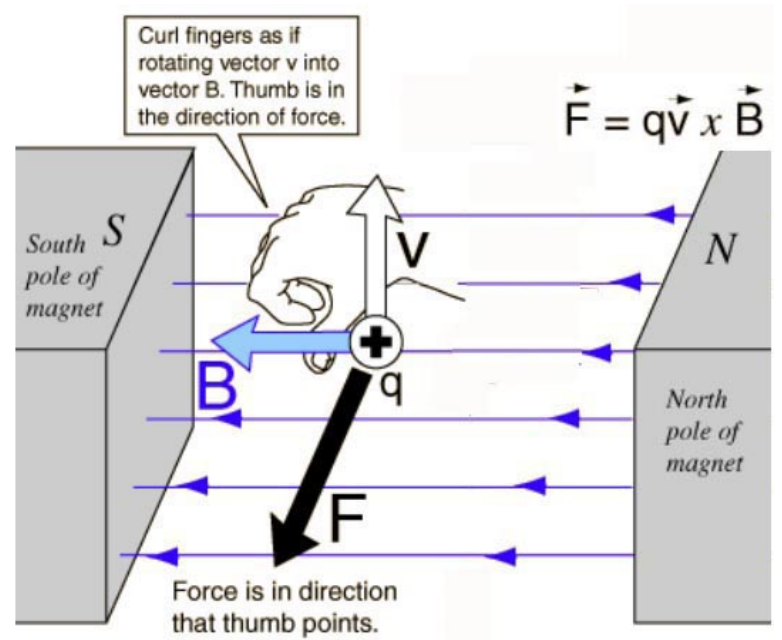

Fig. 13. The Lorentz Force equals the cross product of the Magnetic Induction $B$ and the velocity $v$ of the charge $q$ moving within the magnetic field times the value of the electric charge 
Ampère's Circuital Law represents the electric current density $j$.

$$
\bar{j}=\nabla \times H
$$

Substituting Ampère's Circuital Law (21) in equation (20) results in equation representing the magnetic force density f $f_{\text {LORENTZ: }}$

$$
\begin{aligned}
& \bar{j}=(\nabla \times \mathrm{H}) \quad\left[\mathrm{A} / \mathrm{m}^{2}\right] \\
& \mathrm{f}_{\text {MAGNETIC }}=\overline{\mathrm{f}}_{\text {LORENTZ }}=-\mu \overline{\mathrm{H}} \times(\overline{\mathrm{j}})=-\mu \overline{\mathrm{H}} \times(\nabla \times \overline{\mathrm{H}})\left[\mathrm{N} / \mathrm{m}^{3}\right]
\end{aligned}
$$




\subsubsection{The Fundamental Equation for the Electromagnetic field}

Newton's second law of motion applied within any arbitrary electromagnetic field configuration results in the fundamental equation (23) for any arbitrary electromagnetic field configuration (a beam of light):

$$
\begin{aligned}
& \text { NEWTON: } \mathrm{F}_{\text {TOTAAL }}=\mathrm{m} \text { a represents: } \mathrm{f}_{\text {TOTAAL }}=\rho \mathrm{a} \\
& -\rho \mathrm{a}+\mathrm{f}_{\text {TOTALL }}=0 \\
& -\rho \mathrm{a}+\mathrm{f}_{\text {ELEKTRISCH }}+\mathrm{f}_{\text {MAGNETISCH }}=0 \\
& -\rho \mathrm{a}+\mathrm{F}_{\text {COULOMB }}+\mathrm{F}_{\text {LORentz }}+\mathrm{F}_{\text {COULOMB }}+\mathrm{F}_{\text {Lorentz }}=0 \\
& -\frac{1}{c^{2}} \frac{\partial(\overline{\mathrm{E}} \times \overline{\mathrm{H}})}{\partial t}+\varepsilon_{0} \overline{\mathrm{E}}(\nabla \cdot \overline{\mathrm{E}})-\varepsilon_{0} \overline{\mathrm{E}} \times(\nabla \times \overline{\mathrm{E}})+\mu_{0} \overline{\mathrm{H}}(\nabla \cdot \overline{\mathrm{H}})-\mu_{0} \overline{\mathrm{H}} \times(\nabla \times \overline{\mathrm{H}})=0 \\
& \begin{array}{lllll}
\text { B-1 } & \text { B-2 } & \text { B-3 } & \text { B-4 } & \text { B-5 }
\end{array}
\end{aligned}
$$

Term B-4 is the magnetic representation of the (electric) Coulomb's law B-2 and Term B-3 is the electric representation of the (magnetic) Lorentz Force. 


\subsection{The Fundamental Equation for the Black Hole, describing Gravitational-Electromagnetic Interaction}

The fundamental equation for a Black Hole describes the interaction between light and gravity. To define the Fundamental Equation for the Black Hole, describing the interaction of Gravity on an electromagnetic field, an extra term (B-6) has been introduced in equation (23). The term B-6 represents the force density of the gravitational field acting on the electromagnetic mass density.

$$
\mathrm{F}_{\text {GRAVITY }}=\mathrm{m} \overline{\mathrm{g}}[\mathrm{N}]
$$

Dividing both terms by the Volume V:

$\frac{\mathrm{F}_{\text {GRAVITY }}}{\mathrm{V}}=\frac{\mathrm{m}}{\mathrm{V}} \overline{\mathrm{g}}\left[\mathrm{N} / \mathrm{m}^{3}\right]$

Results in the force density:

$\mathrm{f}_{\text {GRAVITY }}=\rho \overline{\mathrm{g}} \quad\left[\mathrm{N} / \mathrm{m}^{3}\right]$

The specific mass " $\rho$ " of a beam of light follows from Einstein's equation:

$$
\mathrm{W}=\mathrm{m} \mathrm{c}^{2}
$$

Divinding both terms by the Volume $\mathrm{V}$ results in:

$$
\frac{\mathrm{W}}{\mathrm{V}}=\frac{\mathrm{m}}{\mathrm{V}} \mathrm{c}^{2}
$$

which represents the energy density $\mathrm{w}$ and the specific mass $\rho$ of the electromagnetic radiation:

$\mathrm{w}=\rho \mathrm{c}^{2}$

which results for an expression of the specific mass $\rho$ :

$$
\rho=\frac{1}{\mathrm{c}^{2}} \mathrm{~W}=\varepsilon \mu \mathrm{W}
$$


The energy density " $w$ " follows from the electric and the magnetic field intensities:

$$
\begin{aligned}
& \mathrm{w}=\frac{1}{2} \varepsilon \mathrm{E}^{2}+\frac{1}{2} \mu \mathrm{H}^{2} \\
& \mathrm{w}=\frac{1}{2}\left(\varepsilon \mathrm{E}^{2}+\mu \mathrm{H}^{2}\right)=\frac{1}{2}(\varepsilon(\overline{\mathrm{E}} \cdot \overline{\mathrm{E}})+\mu(\overline{\mathrm{H}} \cdot \overline{\mathrm{H}}))
\end{aligned}
$$

Substituting equation (26) in equation (17) results in the gravitational force density f fRAVITY acting on an arbitrary electromagnetic field configuration (a beam of light) with mass density $\rho$.

$$
\begin{aligned}
& \mathrm{f}_{\text {GRAVITY }}=\rho \overline{\mathrm{g}} \\
& \mathrm{f}_{\text {GRAVITY }}=\rho \overline{\mathrm{g}}=\varepsilon \mu \mathrm{w} \overline{\mathrm{g}}=\frac{1}{2}\left(\varepsilon^{2} \mu(\overline{\mathrm{E}} \cdot \overline{\mathrm{E}})+\varepsilon \mu^{2}(\overline{\mathrm{H}} \cdot \overline{\mathrm{H}})\right) \overline{\mathrm{g}}
\end{aligned}
$$

Substituting equation (27) in equation (23) results in the fundamental equation describing the Electromagnetic-Gravitational interaction for any arbitrary electromagnetic field configuration (a beam of light):

$$
\begin{aligned}
& \text { NEWTON: } \overline{\mathrm{F}}_{\text {TOTAAL }}=\mathrm{m} \overline{\mathrm{a}}[\mathrm{N}] \\
& \text { NEWTON: Expressed in force densities: } \overline{\mathrm{f}}_{\text {TOTAAL }}=\rho \overline{\mathrm{a}}\left[\mathrm{N} / \mathrm{m}^{3}\right] \\
& -\rho \overline{\mathrm{a}}+\overline{\mathrm{f}}_{\text {TOTAAL }}=\overline{0} \\
& -\rho \overline{\mathrm{a}}+\overline{\mathrm{f}}_{\text {ELERTRISCH }}+\overline{\mathrm{f}}_{\text {MAGNETISCH }}+\overline{\mathrm{f}}_{\text {GRAVITY }}=\overline{0} \\
& \overline{\mathrm{f}}_{\text {INERTIA }}+\overline{\mathrm{f}}_{\text {COULOMB }}+\overline{\mathrm{f}}_{\text {LORENTZ }}+\overline{\mathrm{f}}_{\text {COULOMB }}+\overline{\mathrm{f}}_{\text {LORENTZ }}+\overline{\mathrm{f}}_{\text {Gravity }}=\overline{0} \\
& \left.-\frac{1}{c^{2}} \frac{\partial(\overline{\mathrm{E}} \times \overline{\mathrm{H}})}{\partial t}+\varepsilon_{0} \overline{\mathrm{E}}(\nabla \cdot \overline{\mathrm{E}})-\varepsilon_{0} \overline{\mathrm{E}} \times(\nabla \times \overline{\mathrm{E}})+\mu_{0} \overline{\mathrm{H}}(\nabla \cdot \overline{\mathrm{H}})-\mu_{0} \overline{\mathrm{H}} \times(\nabla \times \overline{\mathrm{H}})+\frac{1}{2}\left(\varepsilon^{2} \mu(\overline{\mathrm{E}} \cdot \overline{\mathrm{E}})+\varepsilon \mu^{2}(\overline{\mathrm{H}} \cdot \overline{\mathrm{H}})\right)\right)^{-}=\overline{0} \\
& \begin{array}{llllll}
\text { B-1 } & \text { B-2 } & \text { B-3 } & \text { B-4 } & \text { B-5 }
\end{array}
\end{aligned}
$$

Term B-1 represents the inertia term of the electromagnetic radiation. Term B-4 is the magnetic representation of the (electric) Coulomb's Force B-2 and Term B-3 is the electric representation of the (magnetic) Lorentz Force B-5. Term B-6 represents the ElectromagneticGravitational interaction of a gravitational field with field acceleration $\bar{g}$ acting on an arbitrary electromagnetic field configuration ( a beam of light) with specific mass $\rho$. 


\subsection{Longitudinal Black Holes}

When a beam of light is approaching a strong gravitational field in the direction of the gravitational field, generated by a Black Hole, the confinement has been called a Longitudinal Black Hole. The direction of propagation of the beam of light is in the same direction (or in the opposite direction) of the gravitational field. According the first term in (1), the beam of light will be accelerated or decelerated. However, the speed of light is a universal constant and for that reason the speed of light cannot increase or decrease. Instead the intensity of the electromagnetic radiation will increase when the beam of light approaches (propagates in the opposite direction as the direction of the gravitational field) the Black Hole. And the intensity of the electromagnetic radiation will decrease when the beam of light leaves (propagates in the same direction as the direction of the gravitational field) the Black Hole.

The Gravitational-Electromagnetic Confinement for the elementary structure beyond the "superstring" is presented in equation (29).

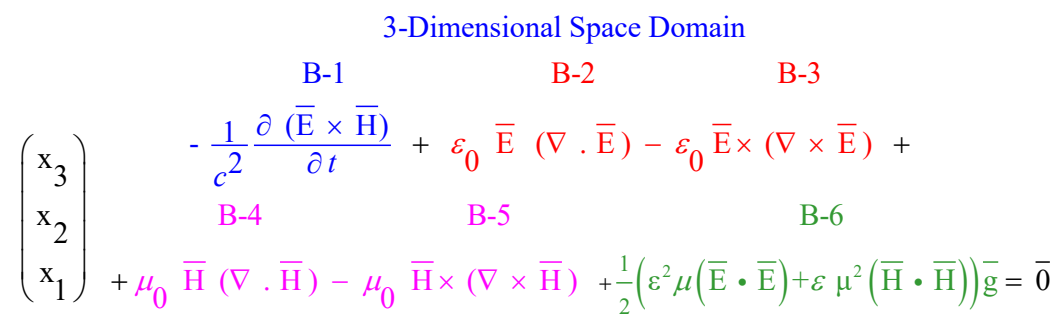

In which $\bar{g}$ represents the gravitational acceleration acting on the the electromagnetic mass density of the confined electromagnetic radiation. 
A possible solution for equation (29) describing an ElectromagneticGravitational confinement within a radial gravitational field with acceleration $\frac{-}{g}$ has been represented in (30).

$$
\begin{aligned}
& \left(\begin{array}{l}
e_{r} \\
e_{\theta} \\
e_{\varphi}
\end{array}\right)=\left(\begin{array}{c}
0 \\
\mathrm{f}(\mathrm{r}) \operatorname{Sin}(\omega \mathrm{t}) \\
-\mathrm{f}(\mathrm{r}) \operatorname{Cos}(\omega \mathrm{t})
\end{array}\right) \quad\left(\begin{array}{l}
m_{r} \\
m_{\theta} \\
m_{\varphi}
\end{array}\right)=\left(\begin{array}{c}
0 \\
\mathrm{f}(\mathrm{r}) \operatorname{Cos}(\omega \mathrm{t}) \\
\mathrm{f}(\mathrm{r}) \operatorname{Sin}(\omega \mathrm{t})
\end{array}\right) \quad \bar{g}=\left(\begin{array}{c}
G_{1} \\
4 \pi \mathrm{r}^{2} \\
0 \\
0
\end{array}\right) \\
& \mathrm{W}_{\mathrm{em}}=\left(\frac{\mu_{0}}{2}(\overline{\mathrm{m}} \cdot \overline{\mathrm{m}})+\frac{\varepsilon_{0}}{2}(\overline{\mathrm{e}} \cdot \overline{\mathrm{e}})\right)=\varepsilon_{0} \mathrm{f}(r)^{2}
\end{aligned}
$$

In which the radial function $\mathrm{f}(\mathrm{r})$ equals:

$$
f[r]=K \mathrm{e}^{-\frac{-\frac{G 1 \varepsilon_{0} \mu_{0}}{r}+8 \pi \log [r]}{8 \pi}}
$$

The solution has been calculated according Newton's Shell Theorem. 


\subsection{Longitudinal Black Hole with an electromagnetic mass of} $10^{-4}[\mathrm{~kg}]$ and a radius $\left.=2 \times 10^{-35}[\mathrm{~m}]\right):$

Figure 14 and Fig. 15 represent the electromagnetic field density (along the vertical axis) as a function of the distance (along the horizontal axis) of the center of the Longitudinal Black Hole with the size of Planck's length. The chosen values equal:

$$
\begin{aligned}
& f[r]=K \mathrm{e}^{-\frac{-\frac{G 1 \mathrm{emm} \varepsilon_{0} \mu_{0}}{r}+8 \pi \log [r]}{8 \pi}} \\
& G 1=6.6740810^{-11} \\
& \mathrm{emm}=10^{-4} \\
& \varepsilon_{0}=8.8510^{-12} \\
& \mu_{0}=1.256637061435917210^{-6}
\end{aligned}
$$

In which "emm" represents the electromagnetic mass of the confinement located at the center according Newton's Shell Theorem.

For an electromagnetic mass emm $=10^{-4}[\mathrm{~kg}]$ of the Longitudinal Black Hole, the radius of the confinement equals approximately $2 \times 10^{-35}[\mathrm{~m}]$ and the first harmonic frequency equals $1.510^{27}[\mathrm{~Hz}]$.

The Plot graph of the Electric Field Intensity $\mathrm{f}(\mathrm{r})$ of the confinement has been presented as a function of the radius in figure (14) and figure (15): 


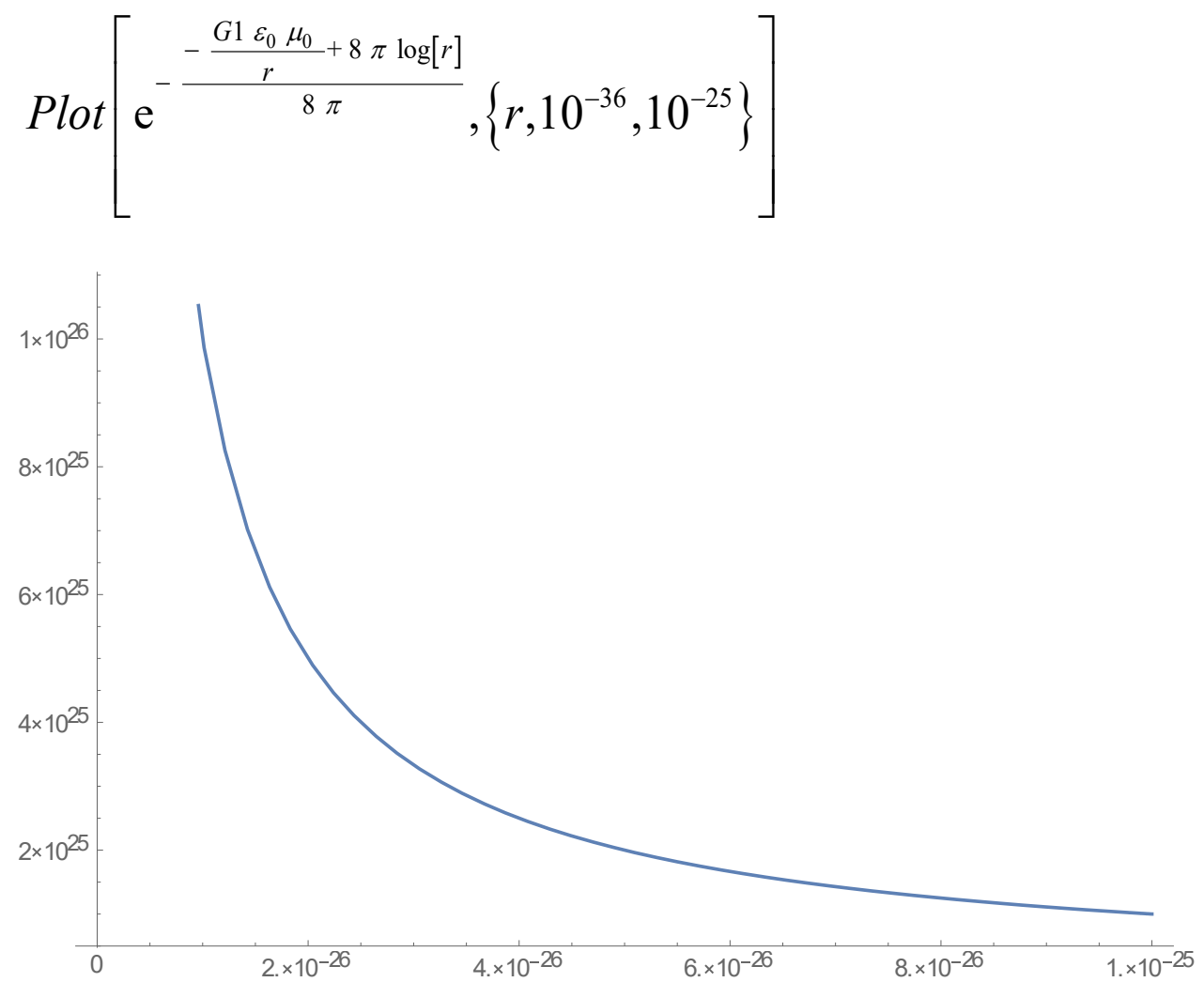

Figure 14 PlotGraph of the Electric Field Intensity $f(r)$ for the region $10^{-36}<\mathrm{r}<10^{-25}$ in which the gravitational field acceleration has been chosen accordingly an electromagnetic mass of $10^{-4}[\mathrm{~kg}]$ located at the center of the confinement, according Newton's Shell Theorem. 


$$
\operatorname{Plot}\left[\mathrm{e}^{-\frac{-\frac{G 1 \varepsilon_{0} \mu_{0}}{r}+8 \pi \log [r]}{8 \pi}},\left\{r, 10^{-36}, 10^{-35}\right\}\right]
$$

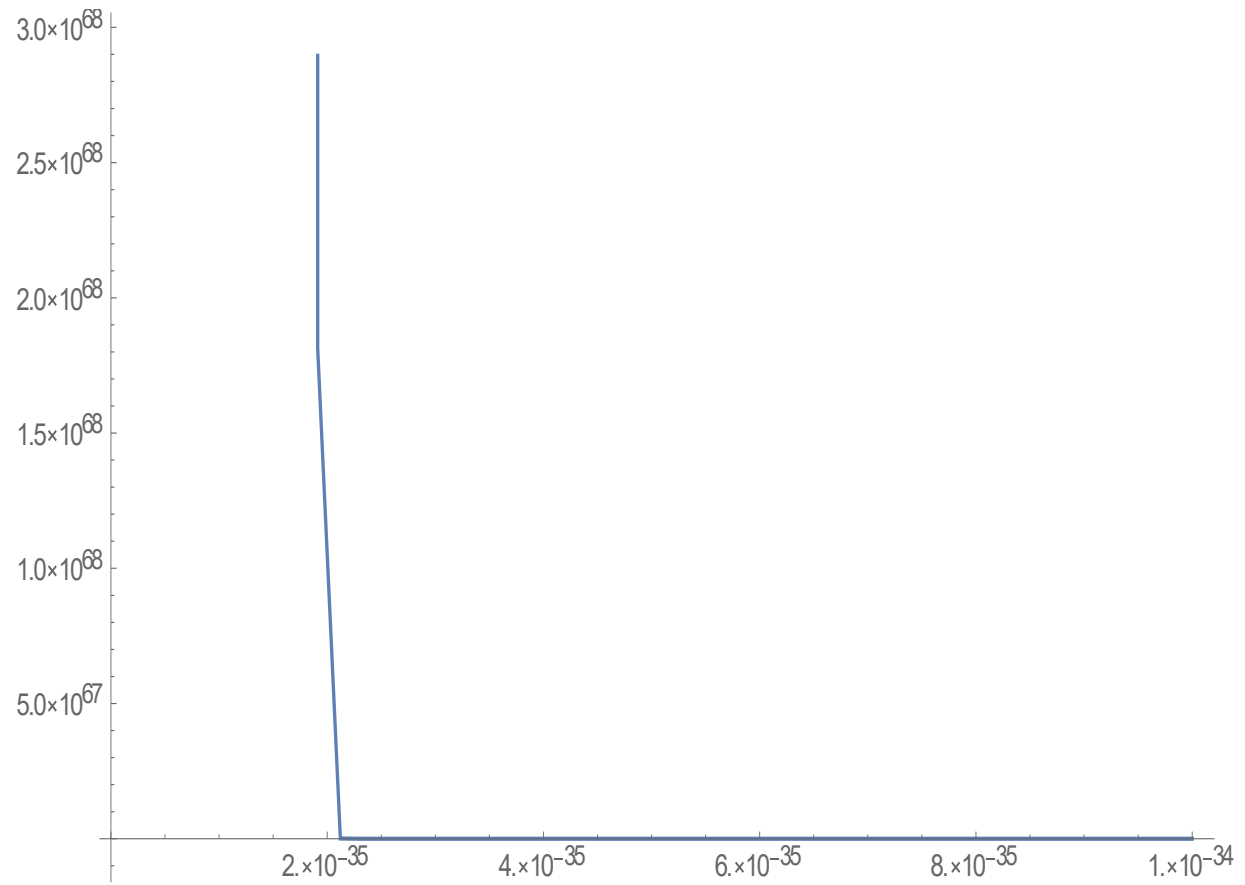

Figure 15 PlotGraph of the Electric Field Intensity $f(r)$ for the region $10^{-36}<\mathbf{r}<10^{-35}$ in which the gravitational field acceleration has been chosen accordingly an electromagnetic mass of $10^{-4}[\mathrm{~kg}]$ located at the center of the confinement, according Newton's Shell Theorem.

It follows from Figure 15 that the radius of the stable gravitational electromagnetic confinement equals approximately $2 \times 10^{-35}[\mathrm{~m}]$, which is the size of the Planck length. According the theory of superstrings, the fundamental constituents of reality are strings of the Planck length (about $1.6210^{-35}[\mathrm{~m}]$ ) that vibrate at resonant frequencies. 


\subsection{Longitudinal Black Hole with an electromagnetic mass of $10^{40}[\mathrm{~kg}]$, a radius $=1.5 \times 10^{9}[\mathrm{~m}]$ at a frequency of $0.2\{\mathrm{~Hz}]$ :}

To realize a Gravitational-Electromagnetic confinement for a conventional Black Hole with an Electro Magnetic Mass: emm $=10^{40}$ [kg], the solution (30) and (31) for the Gravitational Electromagnetic Equilibrium Equation (29) results in a Gravitational Electromagnetic Confinement radius $r=1.510^{9}[\mathrm{~m}]$ (Figure 16 and Figure 17).

$$
\begin{aligned}
& f[r]=K \mathrm{e}^{-\frac{-\frac{G 1 \mathrm{emm} \varepsilon_{0} \mu_{0}}{r}+8 \pi \log [r]}{8 \pi}} \\
& G 1=6.6740810^{-11} \\
& \mathrm{emm}=10^{40} \\
& \varepsilon_{0}=8.8510^{-12} \\
& \mu_{0}=1.256637061435917210^{-6}
\end{aligned}
$$

In which "emm" equals the electromagnetic mass of the Single Harmonic Black Hole located at the center according Newton's Shell Theorem. For an electromagnetic mass of the Single Harmonic Black Hole (SHBH), the value for the electromagnetic mass (emm) equals: emm $=10^{40}[\mathrm{~kg}]$, the radius of the confinement equals approximately $1.5 \times 10^{9}[\mathrm{~m}]$ and the first harmonic frequency equals $0.2[\mathrm{~Hz}]$.

The Plot graph of the Electric Field Intensity $\mathrm{f}(\mathrm{r})$ of the SHBH has been presented as a function of the radius in figure (15) and figure (16): 
$\operatorname{Plot}\left[\mathrm{e}^{-\frac{-\frac{G 1 \varepsilon_{0} \mu_{0}}{r}+8 \pi \log [r]}{8 \pi}},\left\{r, 10^{5}, 10^{12}\right\}\right]$

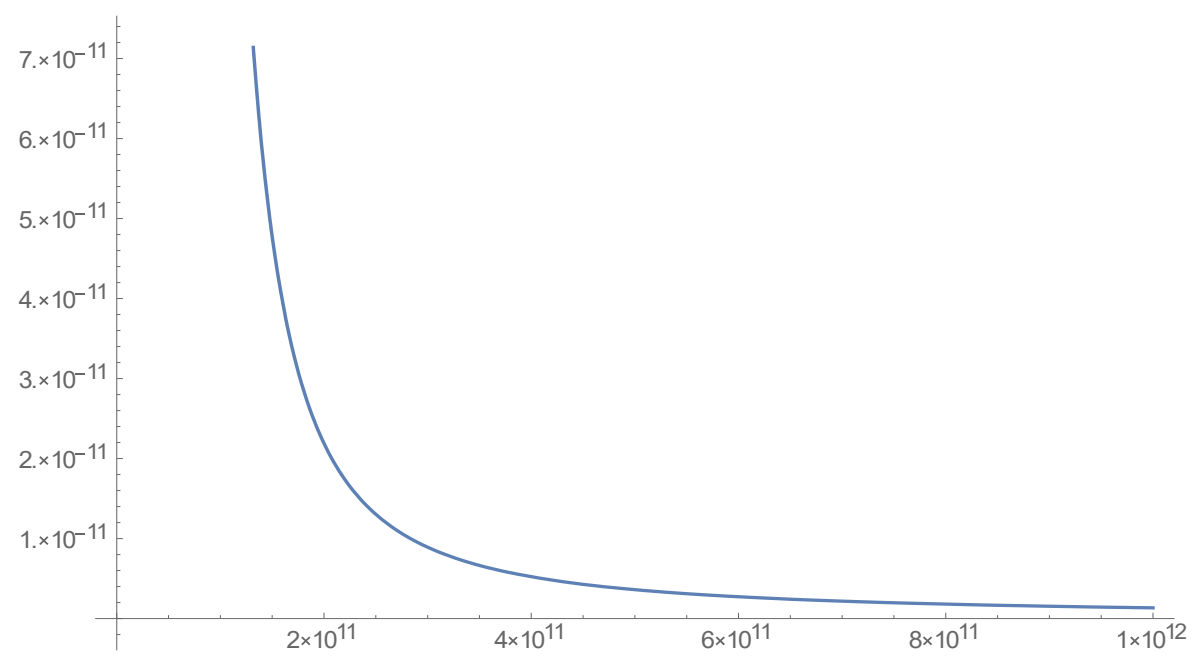

Fig. 16. PlotGraph of the Electric Field Intensity f(r) (vertical axis) for the region $10^{5}<\mathrm{r}<10^{12}$ (horizontal axis) in which the gravitational field acceleration has been chosen accordingly an electromagnetic mass of $10^{40}[\mathrm{~kg}]$ located at the center of the confinement, according Newton's Shell Theorem. 
Plot $\left[\mathrm{e}^{-\frac{-\frac{G 1 \varepsilon_{0} \mu_{0}}{r}+8 \pi \log [r]}{8 \pi}},\left\{r, 10^{9}, 4 \times 10^{9}\right\}\right]$

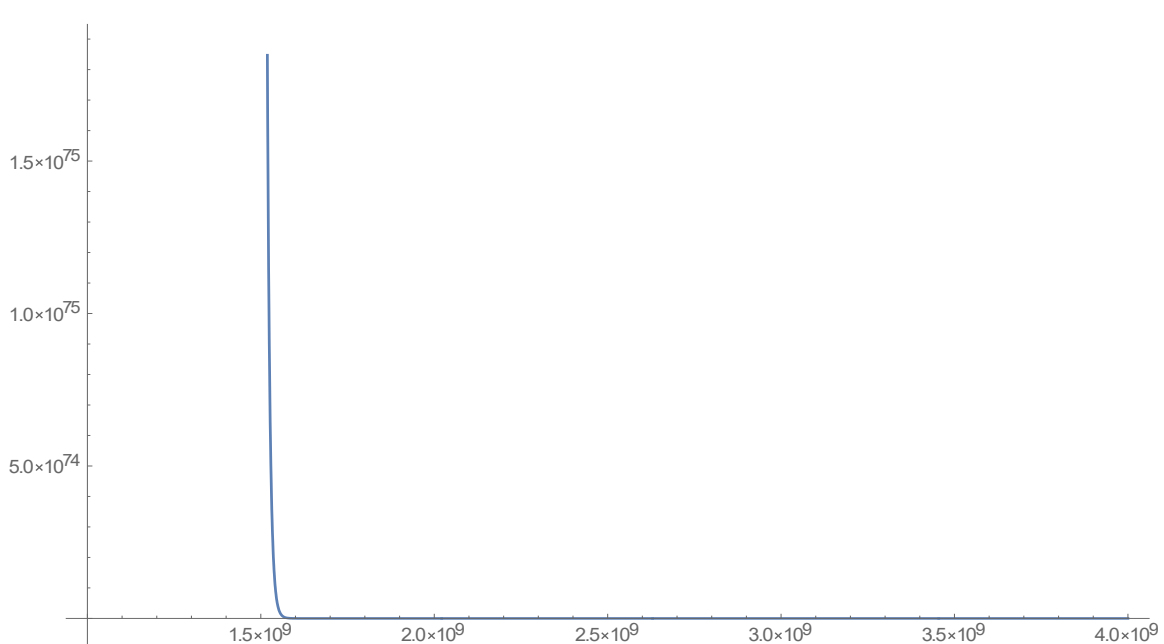

Fig. 17. PlotGraph of the Electric Field Intensity $f(r)$ (vertical axis) for the region $10^{9}<r<410^{9}$ (horizontal axis) in which the gravitational field acceleration has been chosen accordingly an electromagnetic mass of $10^{40}[\mathrm{~kg}]$ located at the center of the confinement, according Newton's Shell Theorem. And a corresponding Single Harmonic frequency of 0.2 [Hz].

It follows from Figure 17 that the radius of the stable gravitational electromagnetic confinement of the SHBH equals approximately $1.510^{9}$ $[\mathrm{m}]$. 


\subsection{Quantum Gravity described by Newton's second law of motion in the $4^{\text {th }}$ dimension (time domain)}

Newton's second law of motion has been described in 3 spatial dimensions, resulting in the fundamental equation for the electromagnetic field.

$$
\begin{gathered}
\text { 3-Dimensional Space Domain } \\
\left(\begin{array}{c}
\mathrm{x}_{3} \\
\mathrm{x}_{2} \\
\mathrm{x}_{1}
\end{array}\right)+\mu_{0} \overline{\mathrm{H}}(\nabla \cdot \overline{\mathrm{H}})-\mu_{0} \overline{\mathrm{H}} \times(\nabla \times \overline{\mathrm{H}})+\frac{1}{2}\left(\varepsilon^{2} \mu(\overline{\mathrm{E}} \cdot \overline{\mathrm{E}})+\varepsilon \mu^{2}(\overline{\mathrm{H}} \cdot \overline{\mathrm{H}})\right) \overline{\mathrm{g}}=\overline{0} \\
\begin{array}{c}
-\frac{1}{c^{2}} \frac{\partial(\overline{\mathrm{E}} \times \overline{\mathrm{H}})}{\partial t}+\varepsilon_{0} \overline{\mathrm{E}}(\nabla \cdot \overline{\mathrm{E}})-\varepsilon_{0} \overline{\mathrm{E}} \times(\nabla \times \overline{\mathrm{E}})+ \\
\mathrm{B}-4
\end{array}
\end{gathered}
$$

It is possible to calculate the $4^{\text {th }}$ dimension of Newton's second law of motion by 4-dimensional vector calculus. The 4-dimensional Electromagnetic Vector Potential has been defined by:

$$
\bar{\varphi}^{4}=\left(\begin{array}{c}
\varphi_{4} \\
\varphi_{3} \\
\varphi_{2} \\
\varphi_{1}
\end{array}\right) \stackrel{\text { CartesianCoordinateSystem }}{\longrightarrow}\left(\begin{array}{c}
\varphi_{\mathrm{t}} \\
\varphi_{\mathrm{z}} \\
\varphi_{\mathrm{y}} \\
\varphi_{\mathrm{x}}
\end{array}\right)
$$

In which the term $\varphi_{\mathrm{a}}$ represents the 4-dimensional electromagnetic vector potential in the "a" direction while the indice "a" varies from 1 to 4 . In a cartesian coordinate system the indices are chosen varying from the $\mathrm{x}, \mathrm{y}, \mathrm{z}$ and $\mathrm{t}$ direction. In which the indice " $\mathrm{t}$ " represents the time direction which has been considered to be the $4^{\text {th }}$ dimension. The 4-dimensional Electromagnetic "Maxwell Tensor" has been defined by:

$$
\mathrm{F}_{a b}=\partial_{b} \varphi_{a}-\partial_{a} \varphi_{b}
$$

Where the indices "a" and "b" vary from 1 to 4 . 
The 4-dimensional Electromagnetic "Energy Momentum Tensor" has been defined by:

$$
T^{a b}=\frac{1}{\mu_{0}}\left[F_{a c} F^{c b}+\frac{1}{4} \delta_{a b} F_{c d} F^{c d}\right]
$$

The 4-dimensional divergence of the 4-dimensional Energy Momentum Tensor equals the 4-dimensional Force Density 4-vector $f^{a}$ :

$$
f^{a}=\partial_{b} \mathrm{~T}^{a b}
$$

Substituting the electromagnetic values for the electric field intensity "E" and the magnetic field intensity " $H$ " in (38) results in the 4dimensional representation of Newton's second law of motion:

$$
\begin{aligned}
& \text { Energy-Time Domain } \\
& \text { B-7 } \\
& \left(\mathrm{f}_{4}\right) \quad \nabla \cdot(\overline{\mathrm{E}} \times \overline{\mathrm{H}})+\frac{1}{2} \frac{\partial\left(\varepsilon_{0}(\overline{\mathrm{E}} \cdot \overline{\mathrm{E}})+\mu_{0}(\overline{\mathrm{H}} \cdot \overline{\mathrm{H}})\right)}{\partial t}=0 \\
& \text { 3-Dimensional Space Domain } \\
& \text { B-1 B-2 } \quad \text { B-3 } \\
& \left(\begin{array}{c}
\mathrm{f}_{3} \\
\mathrm{f}_{2} \\
\mathrm{f}_{1}
\end{array}\right) \begin{array}{c}
-\frac{1}{c^{2}} \frac{\partial(\overline{\mathrm{E}} \times \overline{\mathrm{H}})}{\partial t}+\varepsilon_{0} \overline{\mathrm{E}}(\nabla \cdot \overline{\mathrm{E}})-\varepsilon_{0} \overline{\mathrm{E}} \times(\nabla \times \overline{\mathrm{E}})+ \\
\mathrm{B}-4 \quad \overline{\mathrm{H}}(\nabla \cdot \overline{\mathrm{H}})-\mu_{0} \overline{\mathrm{H}} \times(\nabla \times \overline{\mathrm{H}})+\frac{1}{2}\left(\varepsilon^{2} \mu(\overline{\mathrm{E}} \cdot \overline{\mathrm{E}})+\varepsilon \mu^{2}(\overline{\mathrm{H}} \cdot \overline{\mathrm{H}})\right) \overline{\mathrm{g}}=\overline{0}
\end{array}
\end{aligned}
$$

In which $f_{1}, f_{2}, f_{3}$, represent the force densities in the 3 spatial dimensions and $\mathrm{f}_{4}$ represent the force density in the time dimension $\left(4^{\text {th }}\right.$ dimension). 
The $4^{\text {th }}$ term in equation (39) can be written in the terms of the Poynting vector " $S$ " and the energy density " $w$ " representing the electromagnetic law for the conservation of energy.

$$
\begin{aligned}
& \text { Energy-Time Domain } \\
& \text { Inner Energy } \\
& \text { B-7 } \\
& \left(\mathrm{f}_{4}\right) \\
& \nabla \cdot \overline{\mathrm{S}}+\frac{\partial \mathrm{w}}{\partial t} \quad=0 \\
& \text { 3-Dimensional Space Domain } \\
& \text { B-1 } \\
& \text { B-2 } \\
& \text { B-3 } \\
& \left(\begin{array}{c}
\mathrm{f}_{3} \\
\mathrm{f}_{2} \\
\mathrm{f}_{1}
\end{array}\right)+\begin{array}{c}
-\frac{1}{c^{2}} \frac{\partial(\overline{\mathrm{E}} \times \overline{\mathrm{H}})}{\partial t}+\mu_{0} \overline{\mathrm{H}}(\nabla \cdot \overline{\mathrm{H}})-\mu_{0} \overline{\mathrm{H}} \times\left(\nabla \times \overline{\mathrm{B}}(\nabla \cdot \overline{\mathrm{E}})-\varepsilon_{0} \overline{\mathrm{E}} \times(\nabla \times \overline{\mathrm{E}})+\right. \\
\mathrm{B}-4\left(\varepsilon^{2} \mu(\overline{\mathrm{E}} \cdot \overline{\mathrm{E}})+\varepsilon \mu^{2}(\overline{\mathrm{H}} \cdot \overline{\mathrm{H}})\right) \overline{\mathrm{g}}=\overline{0}
\end{array}
\end{aligned}
$$

The relativistic fundamental equation describing Quantum Physics is the Dirac Equation, which equals the $4^{\text {th }}$ term of Newton's second law of motion.

\section{Energy-Time Domain $(\mathrm{x}-4)$}

$$
\begin{aligned}
& \nabla \cdot(\overline{\mathrm{E}} \times \overline{\mathrm{H}})+\frac{1}{2} \frac{\partial\left(\varepsilon_{0}(\overline{\mathrm{E}} \cdot \overline{\mathrm{E}})+\mu_{0}(\overline{\mathrm{H}} \cdot \overline{\mathrm{H}})\right)}{\partial t} \\
& \bar{\phi} \cdot \overline{\phi^{*}}=\frac{1}{2 \mu}\left(\overline{\mathrm{B}}+\mathrm{i} \frac{\overline{\mathrm{E}}}{c}\right) \cdot\left(\overline{\mathrm{B}}-\mathrm{i} \frac{\overline{\mathrm{E}}}{c}\right)=\frac{1}{2} \mu \mathrm{H}^{2}+\frac{1}{2} \varepsilon \mathrm{E}^{2}=\mathrm{w} \\
& \bar{\phi} \times \overline{\phi^{*}}=\frac{1}{2 \mu}\left(\overline{\mathrm{B}}+\mathrm{i} \frac{\overline{\mathrm{E}}}{c}\right) \times\left(\overline{\mathrm{B}}-\mathrm{i} \frac{\overline{\mathrm{E}}}{c}\right)=\mathrm{i} \sqrt{\varepsilon \mu} \overline{\mathrm{E}} \times \overline{\mathrm{H}}=\mathrm{i} \sqrt{\varepsilon \mu} \overline{\mathrm{S}} \\
& -\frac{i}{\sqrt{\varepsilon_{0} \mu_{0}}} \nabla \cdot(\bar{\phi} \times \bar{\phi})=-\frac{\partial \bar{\phi} \cdot \bar{\phi}^{*}}{\partial t} \\
& \bar{\alpha}=\left[\begin{array}{ll}
0 & \sigma \\
\sigma & 0
\end{array}\right] \text { and } \bar{\beta}=\left[\begin{array}{cc}
\delta_{\alpha b} & 0 \\
0 & -\delta_{\alpha \omega}
\end{array}\right] \\
& \left(\frac{\mathrm{imc}}{h} \bar{\beta}+\bar{\alpha} \cdot \nabla\right) \psi=-\frac{1}{c} \frac{\partial \psi}{\partial t}-\frac{g}{c^{2}} \psi
\end{aligned}
$$

The $4^{\text {th }}$ term of Newton's force density equation (41) can be written as the law for the conservation of electromagnetic energy or can be presented as the relativistic quantum mechanical Dirac Equation. 
In the electromagnetic representation (41) represents the electromagnetic law for the conservation of energy.

$$
\begin{gathered}
\text { Energy-Time Domain }(\mathrm{x}-4) \\
\nabla \cdot(\overline{\mathrm{E}} \times \overline{\mathrm{H}})+\frac{1}{2} \frac{\partial\left(\varepsilon_{0}(\overline{\mathrm{E}} \cdot \overline{\mathrm{E}})+\mu_{0}(\overline{\mathrm{H}} \cdot \overline{\mathrm{H}})\right)}{\partial t}
\end{gathered}
$$

In the quantum physical presentation equation (41) can be written as:

$$
\begin{gathered}
\text { Energy-Time Domain (x-4) } \\
\left(\frac{\mathrm{imc}}{h} \bar{\beta}+\bar{\alpha} \cdot \nabla\right) \psi=-\frac{1}{c} \frac{\partial \psi}{\partial t}-\frac{g}{c^{2}} \psi
\end{gathered}
$$

Which represents the relativistic quantum mechanical Dirac Equation where $\psi$ represents the quantum mechanical probability wave function. The mathematical evidence for the equivalent for (42) and (43) has been published in 1995 in the article: "A Continuous Model of Matter based on AEONs". Equation (1) page 201 to Equation (102) page 213. (Doi: 10.31219/osf.io/ra7ng)

Both equations (42) and (43) are identical but written in a different way.

The Electromagnetic Law for the conservation of Energy (42) and the Relativistic Dirac Equation (43) change within a gravitational field.

As an example, we consider the experiment of a ball being thrown up within a gravitational field. The kinetic energy of the ball at the beginning will be transformed into potential energy at the end until the ball stops moving and falls back to earth.

Something comparable will happen with a beam of light. We consider the experiment of a flashlight, shing up straight from earth in the same direction of the earth as the gravitational field. During the propagation of the light, the kinetic energy of the light will be transformed into potential energy. The kinetic energy of the light is contained by the electromagnetic field. The speed of the light will not change during the process of propagation, but the intensity of the electric field as well the intensity of the magnetic field will decrease with the same amount as 
the potential energy of the beam of light will increase. The potential energy of the beam of light has been expressed by the force density of the gravitational field on the mass density of the beam of light multiplied by the distance. Which has been expressed in equation (44):

$$
\begin{aligned}
& \text { Energy-Time Domain (x-4) } \\
& \nabla \cdot(\overline{\mathrm{E}} \times \overline{\mathrm{H}})+\frac{1}{2} \frac{\partial\left(\varepsilon_{0}(\overline{\mathrm{E}} \cdot \overline{\mathrm{E}})+\mu_{0}(\overline{\mathrm{H}} \cdot \overline{\mathrm{H}})\right)}{\partial t}+\frac{\sqrt{\varepsilon \mu}}{2}(\varepsilon(\overline{\mathrm{E}} \cdot \overline{\mathrm{E}})+\mu(\overline{\mathrm{H}} \cdot \overline{\mathrm{H}})) \overline{\mathrm{g}} \\
& \bar{\phi} \cdot \overline{\phi^{*}}=\frac{1}{2 \mu}\left(\overline{\mathrm{B}}+\mathrm{i} \frac{\overline{\mathrm{E}}}{\mathrm{c}}\right) \cdot\left(\overline{\mathrm{B}}-\mathrm{i} \frac{\overline{\mathrm{E}}}{\mathrm{c}}\right)=\frac{1}{2} \mu \mathrm{H}^{2}+\frac{1}{2} \varepsilon \mathrm{E}^{2}=\mathrm{w} \\
& \bar{\phi} \times \overline{\phi^{*}}=\frac{1}{2 \mu}\left(\overline{\mathrm{B}}+\mathrm{i} \frac{\overline{\mathrm{E}}}{c}\right) \times\left(\overline{\mathrm{B}}-\mathrm{i} \frac{\overline{\mathrm{E}}}{c}\right)=\mathrm{i} \sqrt{\varepsilon \mu} \overline{\mathrm{E}} \times \overline{\mathrm{H}}=\mathrm{i} \sqrt{\varepsilon \mu} \overline{\mathrm{S}} \\
& -\frac{i}{\sqrt{\varepsilon_{0} \mu_{0}}} \nabla \cdot(\bar{\phi} \times \bar{\phi}) \quad=-\frac{\partial \bar{\phi} \cdot \bar{\phi}^{*}}{\partial t} \\
& \bar{\alpha}=\left[\begin{array}{ll}
0 & \sigma \\
\sigma & 0
\end{array}\right] \text { and } \bar{\beta}=\left[\begin{array}{cc}
\delta_{a b} & 0 \\
0 & -\delta_{\alpha b}
\end{array}\right] \\
& \left(\frac{\mathrm{imc}}{h} \bar{\beta}+\bar{\alpha} \cdot \nabla\right) \psi=-\frac{1}{c} \frac{\partial \psi}{\partial t}-\frac{g}{c^{2}} \psi
\end{aligned}
$$

Equation (44) represents as well "The Electromagnetic Law for the conservation of Energy within a Gravitational Field with acceleration g" and the Relativistic Dirac Equation within a Gravitational Field with acceleration g".

The complete fundamental equation describing GravitationalElectromagnetic Interaction within a gravitational field with acceleration "g", is the 4-dimensional representation of Newton's second law of motion represented in equation (45):

$$
\begin{aligned}
& \text { Energy-Time Domain } \\
& \text { Inner Energy Potential Energy } \\
& \text { B-7 } \\
& \text { B-8 } \\
& \left(\mathrm{x}_{4}\right) \quad \nabla \cdot \overline{\mathrm{S}}+\frac{\partial \mathrm{w}}{\partial t}+m \overline{\mathrm{g}} \frac{\Delta \mathrm{x}}{\Delta \mathrm{t}}=0 \\
& \text { 3-Dimensional Space Domain } \\
& \text { B-1 } \\
& \text { B-2 } \\
& \text { B-3 } \\
& \left(\begin{array}{c}
\mathrm{x}_{3} \\
\mathrm{x}_{2} \\
\mathrm{x}_{1}
\end{array}\right)+\begin{array}{c}
-\frac{1}{c^{2}} \frac{\partial(\overline{\mathrm{E}} \times \overline{\mathrm{H}})}{\partial t}+\varepsilon_{0} \overline{\mathrm{H}}\left(\nabla \cdot \overline{\mathrm{E}}(\nabla \cdot \overline{\mathrm{H}})-\mu_{0} \overline{\mathrm{H}} \times(\nabla \times \overline{\mathrm{H}})++\frac{1}{2}\left(\varepsilon^{2} \mu(\overline{\mathrm{E}} \cdot \overline{\mathrm{E}})+\varepsilon \mu^{2}(\overline{\mathrm{H}} \cdot \overline{\mathrm{H}})\right) \overline{\mathrm{g}}=\overline{0}\right. \\
\mathrm{B}-4
\end{array}
\end{aligned}
$$


The fundamental equation, describing "Quantum Gravity" has been represented by the Relativistic Quantum Mechanical Dirac equation with a gravitational field with acceleration $\mathrm{g}$ :

$$
\left(\frac{\mathrm{im} \mathrm{c}}{h} \bar{\beta}+\bar{\alpha} \cdot \nabla\right) \psi=-\frac{1}{c} \frac{\partial \psi}{\partial t}-\frac{g}{c^{2}} \psi
$$

\subsection{Data Availability}

All the Data and all the Calculations to provide evidence to this 'New Theory about Light' have been published in the 'Open Source Framework(OSF)': https://osf.io/gbn4p/

DOI: 10.31219/osf.io/gbn4p ( https://doi.org/10.31219/osf.io/gbn4p )

(Calculations in Mathematica 11.0)', Page 1-33).

\subsection{References}

[1] Oki Gunawana and Yudistira Virgus; The one-dimensional camelback potential in the parallel dipole line trap: Stability conditions and finite size effect; Journal of Applied Physics 121, 133902 (2017); https://doi.org/10.1063/1.4978876

[2] IBM Blog Research; A new effect in electromagnetism discovered

- 150 years later; https://phys.org/news/2017-10-effectelectromagnetism-years.html

[3] J. W. Vegt, A Continuous Model of Matter based on AEONs, Physics Essays ,1995, Volume 8, Number 2, 201-224 A Continuous model of Matter ( https://doi.org/10.31219/osf.io/ra7ng )

[4] Z. K. Minev, S. O. Mundhada, S. Shankar, P. Reinhold, R. GutiérrezJáuregui, R.J. Schoelkopf, N. Mirrahimi, H.J. Carmichael and M. H. Devoret; To catch and reverse a quantum jump mid-flight; Nature; 03 June 2019 equilibrium beyond Einstein 4-Dimensional, KaluzaKlein 5-Dimensional and Superstring 10- and 11 Dimensional Curved Hyperspaces. https://www.nature.com/articles/s41586-019$\underline{1287-\mathrm{Z}}$ 


\section{PREFACE}

This is a book about the world beyond Black Holes and has been divided into three parts.

The first part, Level 1, describes the theory about Black Holes at a fundamental level which is understandable for everyone with a few years of basic Physics education. The reader is familiar with fundamental equations like Newton's second Law of motion $(\mathrm{F}=\mathrm{m}$ a) and Einsteins's famous relationship between mass and energy $\left(\mathrm{E}=\mathrm{m} \mathrm{c}^{2}\right)$.

The second part, Level 2, describes the theory about Black Holes at a university level which is understandable for everyone with a Physics university education. The reader is familiar with fundamental mathematics like tensor calculus.

The third part, Level 3, describes the complete New Theory and offers new insights in the mathematical approach for Black Holes and the relationship with quantum physics, electric charge, magnetic spin and infra-red shift. 


\section{THE WORLD BEYOND BLACK HOLES \\ LEVEL 2}

Light contains the key to open the doors to Heaven. Unfortunately, the same key fits on the doors to Hell

Author:

Country: Website: Email:

\section{Wim Vegt}

The Netherlands

https://wimvegt.topworld.center

j.w.vegt@topacademy.center 


\section{Classical Theory New Theory Newton \\ NEWTON in 3 dimensions \\ Newton \\ NEWTON in 4 dimensions}

$$
\begin{array}{r}
\overline{\mathrm{F}}=\mathrm{m} \overline{\mathrm{a}} \\
\left(\begin{array}{l}
\mathrm{x}_{3} \\
\mathrm{x}_{2} \\
\mathrm{x}_{1}
\end{array}\right) \rightarrow\left(\begin{array}{l}
\mathrm{F}_{\mathrm{z}} \\
\mathrm{F}_{\mathrm{y}} \\
\mathrm{F}_{\mathrm{x}}
\end{array}\right)=\mathrm{m}\left(\begin{array}{l}
\mathrm{a}_{\mathrm{z}} \\
\mathrm{a}_{\mathrm{y}} \\
\mathrm{a}_{\mathrm{x}}
\end{array}\right)
\end{array}
$$

$$
\begin{array}{r}
\overline{\mathrm{F}}^{4}=\mathrm{m} \frac{-4}{\mathrm{a}^{-4}} \\
\left(\begin{array}{l}
\mathrm{x}_{4} \\
\mathrm{x}_{3} \\
\mathrm{x}_{2} \\
\mathrm{x}_{1}
\end{array}\right) \rightarrow\left(\begin{array}{l}
\mathrm{F}_{\mathrm{t}} \\
\mathrm{F}_{\mathrm{z}} \\
\mathrm{F}_{\mathrm{y}} \\
\mathrm{F}_{\mathrm{x}}
\end{array}\right)=\mathrm{m}\left(\begin{array}{l}
\mathrm{a}_{\mathrm{t}} \\
\mathrm{a}_{\mathrm{z}} \\
\mathrm{a}_{\mathrm{y}} \\
\mathrm{a}_{\mathrm{x}}
\end{array}\right)
\end{array}
$$

Maxwell (Newton in 3 Dimensions)

$$
\begin{aligned}
& \nabla \cdot \overline{\mathrm{E}}=\frac{\rho}{\varepsilon} \\
& \nabla \times \overline{\mathrm{E}}=-\mu \frac{\partial \overline{\mathrm{H}}}{\partial \mathrm{t}} \\
& \nabla \cdot \overline{\mathrm{H}}=0 \\
& \nabla \times \overline{\mathrm{H}}=\varepsilon \frac{\partial \overline{\mathrm{E}}}{\partial \mathrm{t}}
\end{aligned}
$$

Dirac/ Schrôdinger (Newton $4^{\text {th }}$ Dimension)

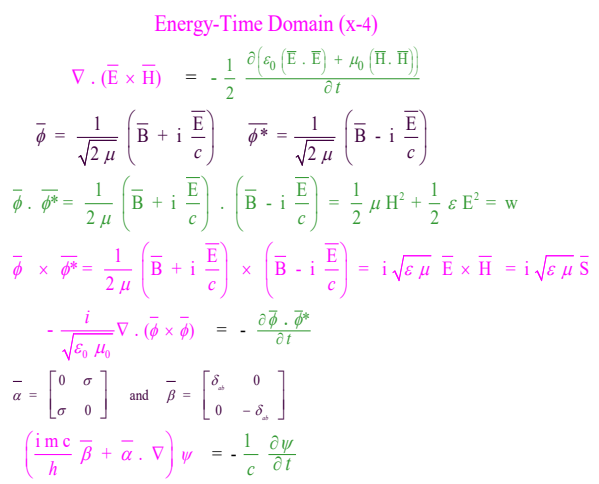

Quantum Gravity (Newton $4^{\text {th }}$ Dimension)

Energy-Time Domain (x-4)

$\nabla \cdot(\overline{\mathrm{E}} \times \overline{\mathrm{H}})+\frac{1}{2} \frac{\partial \varepsilon_{0}(\overline{\mathrm{E}} \cdot \overline{\mathrm{E}})+\mu_{0}(\overline{\mathrm{H}} \cdot \overline{\mathrm{H}})}{\partial t}+\frac{\sqrt{\varepsilon \mu}}{2}(\varepsilon(\overline{\mathrm{E}} \cdot \overline{\mathrm{E}})+\mu(\overline{\mathrm{H}} \cdot \overline{\mathrm{H}})) \bar{g}$

$\bar{\phi} \cdot \overline{\phi^{*}}=\frac{1}{2 \mu}\left(\overline{\mathrm{B}}+\mathrm{i} \frac{\overline{\mathrm{E}}}{c}\right) \cdot\left(\overline{\mathrm{B}}-\mathrm{i} \frac{\overline{\mathrm{E}}}{c}\right)=\frac{1}{2} \mu \mathrm{H}^{2}+\frac{1}{2} \varepsilon \mathrm{E}^{2}=\mathrm{w}$

$\bar{\phi} \times \overline{\phi^{*}}=\frac{1}{2 \mu}\left(\overline{\mathrm{B}}+\mathrm{i} \frac{\overline{\mathrm{E}}}{c}\right) \times\left(\overline{\mathrm{B}}-\mathrm{i} \frac{\overline{\mathrm{E}}}{c}\right)=\mathrm{i} \sqrt{\varepsilon \mu} \overline{\mathrm{E}} \times \overline{\mathrm{H}}=\mathrm{i} \sqrt{\varepsilon \mu} \overline{\mathrm{S}}$

$-\frac{i}{\sqrt{\varepsilon_{0} \mu_{0}}} \nabla \cdot(\bar{\phi} \times \bar{\phi})=-\frac{\partial \bar{\phi} \cdot \bar{\phi}^{*}}{\partial t}$

$\bar{\alpha}=\left[\begin{array}{ll}0 & \sigma \\ \sigma & 0\end{array}\right]$ and $\bar{\beta}=\left[\begin{array}{cc}\delta_{\infty} & 0 \\ 0 & -\delta_{\infty}\end{array}\right]$

$\left(\frac{\mathrm{imc}}{h} \bar{\beta}+\bar{\alpha} \cdot \nabla\right) \psi=-\frac{1}{c} \frac{\partial \psi}{\partial t}-\frac{g}{c^{2}} \psi$

$$
\begin{aligned}
& \text { NEWTON: } \rightarrow \overline{\mathrm{F}}_{\text {TOTAL }}=\mathrm{m} \overline{\mathrm{a}}[\mathrm{N}] \rightarrow \overline{\mathrm{f}}_{\text {TOTAL }}=\rho \overline{\mathrm{a}}\left[\mathrm{N} / \mathrm{m}^{\mathrm{3}}\right] \\
& -\rho \overline{\mathrm{a}}+\quad \overline{\mathrm{f}}_{\text {ToTAL }}\left[\overline{0}\left[\mathrm{~N} / \mathrm{m}^{3}\right]\right. \\
& -\rho \overline{\mathrm{a}}+\overline{\mathrm{f}}_{\text {HLCTRRC }}+\overline{\mathrm{f}}_{\text {MAGNetiC }}=\overline{0}\left[\mathrm{~N} / \mathrm{m}^{3}\right] \\
& -\rho \overline{\mathrm{a}}+\overline{\mathrm{f}}_{\text {COLLONB }}+\overline{\mathrm{f}}_{\text {LORENTZ }}+\overline{\mathrm{f}}_{\text {COLLOMB }}+\overline{\mathrm{f}}_{\text {LORRNZZ }}=\overline{0}\left[\mathrm{~N} / \mathrm{m}^{3}\right] \\
& -\frac{1}{c^{2}} \frac{\partial(\mathrm{E} \times \mathrm{H})}{\partial t}+\varepsilon_{0} \overline{\mathrm{E}}(\nabla \cdot \overline{\mathrm{E}})-\varepsilon_{0} \overline{\mathrm{E}} \times(\nabla \times \overline{\mathrm{E}})+\mu_{0} \overline{\mathrm{H}}(\nabla \cdot \overline{\mathrm{H}})-\mu_{0} \overline{\mathrm{H}}_{\times} \times(\nabla \times \overline{\mathrm{H}})=0\left[\mathrm{~N} / \mathrm{m}^{3}\right]
\end{aligned}
$$

Dirac/ Schrődinger (Newton $4^{\text {th }}$ Dimension)

$$
\begin{aligned}
& \text { Energy-Time Domain (x-4) } \\
& \text { B-6 B-7 } \\
& \nabla \cdot(\overline{\mathrm{E}} \times \overline{\mathrm{H}})=-\frac{1}{2} \frac{\partial\left(\varepsilon_{0}(\overline{\mathrm{E}} \cdot \overline{\mathrm{E}})+\mu_{0}(\overline{\mathrm{H}} \cdot \overline{\mathrm{H}})\right)}{\partial t} \\
& \text { 3-Dimensional Space Domain } \\
& \text { B-1 B-2 } \quad \text { B-3 } \\
& \left(\begin{array}{c}
\mathrm{x}_{3} \\
\mathrm{x}_{2} \\
\mathrm{x}_{1}
\end{array}\right) \quad \begin{array}{c}
-\frac{1}{c^{2}} \frac{\partial(\overline{\mathrm{E}} \times \overline{\mathrm{H}})}{\partial t}+\varepsilon_{0} \overline{\mathrm{E}}(\nabla \cdot \overline{\mathrm{E}})-\varepsilon_{0} \overline{\mathrm{E}} \times(\nabla \times \overline{\mathrm{E}})+ \\
\mathrm{B}-4 \quad \\
+\mu_{0} \overline{\mathrm{H}}(\nabla \cdot \overline{\mathrm{H}})-\mu_{0} \overline{\mathrm{H}} \times(\nabla \times \overline{\mathrm{H}})=\overline{0}
\end{array}
\end{aligned}
$$

Quantum Gravity (Newton $4^{\text {th }}$ Dimension)

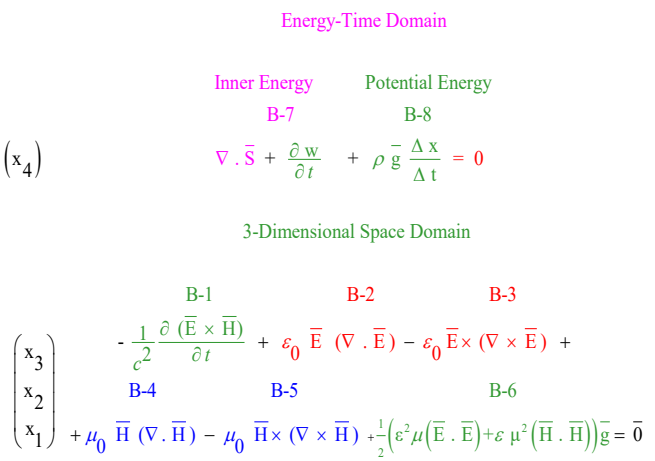




\subsection{Black Holes Level 2}

\section{Index}

2.1 Introduction $\underline{65}$

2.2 Impact of a New Theory on Modern Physics $\underline{66}$ from the last 150 years

2.3 Single Harmonic Black Holes

2.4 The Inner structure of the photon

2.5 The Unified 4-Dimensional Hyperspace

Equilibrium Equation (Newton's Law in 4 Dimensions):

2.6 Fundamental Solutions of the Unified 4- $\quad \underline{80}$ Dimensional Hyperspace Equilibrium Equation

2.7 The propagation of a beam of light in one single $\underline{81}$ direction with an arbitrary electromagnetic intensity division in the plane perpendicular to the direction of propagation:

2.8 The pre-eminence of the Unified 4-Dimensional $\underline{82}$ Hyperspace Equilibrium Equation compared to the classical 4 Maxwell equations:

2.9 Electromagnetic Mass (Inertia):

2.10 The Origin of Electromagnetic Mass (Inertia):

2.11 The radiation pressure on Mirror B when Mirror $B$ moves with a velocity $\mathrm{v}$ in the direction of the positive $\mathrm{z}$-axis

2.12 Newton's second Law of Motion (Inertia) for Confined Electromagnetic Radiation

2.13 Longitudinal Mass. The Real Light Intensity of 91 the Sun, measured in our Solar: System, including Electromagnetic Gravitational Conversion (EMGC)

2.14 Transversal Electromagnetic Mass (Inertia)/ $9 \underline{95}$ Confined Mass (The Impact of Gravity on Light)

2.15 The Physical properties (inertia, mass, momentum, velocity) of the Photon Electromagnetic-Gravitational Interaction in a non-linear Space-Time Continuum

2.16 Gravitational-Electromagnetic Confinement 
2.17 Gravitational-Electromagnetic Confinement: $\quad \underline{106}$

Type $1(\mathrm{emm}=10-4[\mathrm{~kg}], \mathrm{r}=2$ 10-35 [m])

2.18 Gravitational-Electromagnetic Confinement:

$\underline{109}$

Type $2(\mathrm{emm}=10-12[\mathrm{~kg}], \mathrm{r}=2.510-43[\mathrm{~m}])$

2.19 Gravitational-Electromagnetic Confinement:

Type $3(\mathrm{emm}=10-27[\mathrm{~kg}], \mathrm{r}=3$ 10-58 [m])

2.20 The Origin of Electric Charge and Magnetic

Spin in Discrete Values

2.21 Types of "Sub Max Planck Length

Gravitational-Electromagnetic Confinements" resulting in Electric Charge and Magnetic Spin

2.21.1 Type 1 of "Sub Max Planck Length

Gravitational-Electromagnetic Confinement"

(Electric- and Magnetic Dipoles, Electric- and

Magnetic Spin) $\{\mathrm{m} 1=0, \mathrm{n} 1=0, \mathrm{p} 1=0, \mathrm{q} 1=0\}$

$\left(\mathrm{emm}=10^{-4}[\mathrm{~kg}]\right.$; radius $\left.=2 \times 10^{-35}[\mathrm{~m}]\right)$ :

2.21.2 Type 2 of "Sub Max Planck Length

Gravitational-Electromagnetic Confinements"

(Electric- and Magnetic Dipoles, Electric- and Magnetic Spin) $\{\mathrm{m} 1=1, \mathrm{n} 1=0, \mathrm{p} 1=0, \mathrm{q} 1=0\}$

2.21.3 Type 3 of "Sub Max Planck Length

Gravitational-Electromagnetic Confinements" $\{\mathrm{m} 1=1, \mathrm{n} 1=1, \mathrm{p} 1=0, \mathrm{q} 1=0\}$

2.21.4 Type 4 of "Sub Max Planck Length

Gravitational-Electromagnetic Confinements"

(Electric- and Magnetic Dipoles, Electric- and

Magnetic Spin) $\{\mathrm{m} 1=0, \mathrm{n} 1=0, \mathrm{p} 1=1, \mathrm{q} 1=0\}$

2.21.5 Type 5 of "Sub Max Planck Length

Gravitational-Electromagnetic Confinements" $\{\mathrm{m} 1=0, \mathrm{n} 1=0, \mathrm{p} 1=1, \mathrm{q} 1=1\}$

2.22 Type 2 of "Sub Max Planck Length

Gravitational-Electromagnetic Confinements"

2.23 Single Harmonic Black Holes (SHBH)

2.24 Single Harmonic Black Hole Type $1(\mathrm{emm}=$ $1040[\mathrm{~kg}], \mathrm{r}=1.5109[\mathrm{~m}], \mathrm{f}=0.2[\mathrm{~Hz}])$

2.25 Conclusions

2.26 Data Availability

2.27 References 


\subsection{Introduction}

To understand the physics of Black Holes, it is important to understand the first law in physics which controls our whole universe. This is the law of "Perfect Equilibrium". Within the whole universe always does exist a prefect equilibrium between all the physical forces like gravity, forces of inertia, radiation pressure and Electro-Magnetic Interaction forces at any time, in any direction and at any space coordinate. This is the fundamental law in physics on which also the existence of a Black Hole has been grounded.

This new theory will explain the forces within a beam of light interacting with gravity while the beam of light propagates within the gravitational field generated by a black hole. 


\subsection{Impact of a New Theory on Modern Physics from the last 150 years}

When we look at Modern Physics developed during the last 150 years, it is clearly to recognize fundamental important turning points during the last 150 years. A great and important turning point has been the fifth Solvay Conference in October 1927 where a fundamental debate took place between Niels Bohr (representing the instrumentalists) and Albert Einstein (representing the scientific realists). The instrumentalists wanted loser rules based on outcomes. The outcome of this fundamental debate between Niels Bohr and Albert Einstein was that the instrumentalists won the debate. Based on this outcome, the fundamental quantum physical "Copenhagen Interpretation", had been presented by Niels Bohr and and Werner Heisenberg and was generally accepted.

The fundamental question is: Would the development of the fundamental theories in Modern Physics had been different when Maxwell would have found a different set of equations for the electromagnetic field in his famous and well-known publication in 1865 "A Dynamical Theory of the Electromagnetic Field".

And indeed, the final and only conclusion will be that physics would have developed in a totally different way when Maxwell would have found a different set of equations, describing the electromagnetic field. When Maxwell would have found equation (5), presented in this book, the New Equation for the Electromagnetic field, the name "Quantum Physics" would probably never exist and the "Copenhagen Interpretation" would never have been formulated by Niels Bohr and Werner Heisenberg. The new name for Quantum Physics" would probably be something like "Quantum Electrodynamics" based on the fundamental properties of the electromagnetic field.

Because one of the possible outcomes (solutions) of equation (5) is the possibility of independently and separately existing "Electromagnetic Fields Configurations" confined by their own electromagnetic mutual interaction. Existing like elementary particles with their own distinctive frequencies and interacting like non deformable particles. 
The underlaying conflict in the famous discussion between Niels Bohr and Albert Einstein at the fifth Solvay Conference in October 1927 was the impossibility to explain the matter waves proposed by Louis de Broglie within an electromagnetic concept.

The concept that matter behaves like a wave was proposed by Louis de Broglie, who introduced in 1924 the concept of "matter waves". Erwin Schrödinger published in 1926 the famous quantum mechanical Schrödinger wave equation. The Schrödinger wave equation formulated the "matter waves" proposed by "Louis de Broglie" within a mathematical concept.

Since then fundamental choices have been made, finally resulting in the "Copenhagen Interpretation" formally presented during the fifth Solvay Conference in October 1927 by Niels Bohr and Werner Heisenberg.

However, the well-known "Copenhagen Interpretation" has been a direct consequence of the wrong formulation of the Maxwell equations. Because the mathematical solutions for the Schrödinger wave equation" are complex solutions, describing spherical and elliptical waves.

It is impossible to find with the classical Maxwell equations the complex spherical and elliptical wave solutions which were found with the Schrödinger wave equation. And for that reason it was clearly impossible that matter waves could be electromagnetic waves. And a totally new kind of waves, indicated as "matter waves", had to be introduced.

In this book, equation (5) has been introduced, describing the electrodynamics of the electromagnetic field. And this equation (5) easily results in the spherical solutions, represented in equation (42), and the elliptical complex solutions. And for that reason electromagnetic waves are still a fundamental and important candidate for the quantum mechanical "matter waves".

Confined electromagnetic waves represent the property of inertia and like every kind of matter obey Newton's second law: $F=$ m.a, presented in equation (36). This is a second argument to choose for confined electromagnetic waves as an important candidate for "matter waves" 
Introducing two electromagnetic complex functions (5.1.1) and (5.1.2) and substituting both complex electromagnetic functions in the New Electromagnetic Equation 5.1 results in the quantum mechanical Dirac equation $(5.1 .7)$ which is a relativistic equivalent for the quantum mechanical Schrödinger wave equation. This is the third argument to choose for confined electromagnetic waves as an important candidate for "matter waves"

The 1927 Solvay Conference has been a fundamental and determining point in the history of Modern Physics. From that starting point the "quantum mechanical "Copenhagen Interpretation" has been the fundamental " Leitmotiv " in modern physics.

But this "Leitmotiv" has been based on the wrong equation. Because the Maxwell Equations are linear equations and do not have the fundamental mathematical complex solutions like the quantum mechanical Dirac equation and the Schrödinger wave equation. And for that fundamental reason "electromagnetic waves" could never be a candidate for the till then unknown "matter waves" or the "de Broglie" waves.

With the presentation of the new equation (5) a new set of fundamental mathematical complex solutions for confined electromagnetic waves has been offered. Solutions which match the mathematical solutions for the quantum mechanical Dirac Equation and the Schrödinger wave equation.

Introducing in this way a new "Leitmotiv " in Modern Physics based on the fundamental new concept that "Confined Electromagnetic Waves" with the mechanical properties of mass and momentum are a serious candidate for the "matter waves" or the "De Broglie waves". In this new concept the whole idea of "Probability Waves" combined with the "Wave-Particle Duality" will disappear. 


\subsection{Single Harmonic Black Holes}

In this New Theory a "Single Harmonic Black Hole" (SHBH) has been considered to be the Gravitational-Electromagnetic Confinement of a Single Harmonic Electromagnetic Field Configuration in which a perfect equilibrium exists between the outward directed electromagnetic radiation pressure and the inward directed ElectromagneticGravitational Interaction force densities. This frequency transformation is possible because of the combined Lorentz / Doppler-Effect transformation during the collapse (contraction) of the radiation when the Gravitational Electromagnetic Confinement has been formed (Implosion of Visible Light). Within the scope of this article "Single Harmonic Black Hole" (SHBH) is considered to be any kind of 3dimensional confined Single Harmonic Electromagnetic Energy. The inner structure of a "SHBH" has been based on a 3-dimensional isotropic equilibrium within the electromagnetic field configuration. This new theory will explain how electromagnetic fields (wave packages) demonstrate inertia, mass and momentum and which forces keep the wave packages together in a way that they can be measured like particles with their own specific mass and momentum. To understand what electromagnetic inertia and the corresponding electromagnetic mass, spin and electric charge is and how the anisotropy of electromagnetic mass, spin and electric charge can be explained and how it has to be defined, a New Theory about "Electromagnetic-Gravitational Interaction" has been developed. The "New Theory" has been based on the fundamental principle of "Perfect Equilibrium within the Universe" which has already been expressed by Newton's three equations published in 1687 in "Philosophiae Naturalis Principia Mathematica. Newton's Equations in 3 dimensions will be published in this article in an extension into 4 dimensions. Newton's 4-dimensional law in the 3 spatial dimensions results in an improved version of the classical Maxwell Equations and Newton's law in the 4th dimension (time) results in the quantum mechanical Schrödinger wave equation (at nonrelativistic velocities) and the relativistic Dirac equation. 


\subsection{The Inner structure of the photon}

The inner structure of a photon is based on a 3-dimensional anisotropic equilibrium within the electromagnetic pulses in which an equilibrium does exist for the Electric and the Magnetic Fields separately generated by the pulses. A photon cannot be considered as a particle. Because particles are 3-dimensional confinements. Photons are anisotropic (in $1^{\text {st }}$ and $2^{\text {nd }}$ dimension a particle and in the $3^{\text {rd }}$ dimension a wave) confinements of electromagnetic pulses, generated during the energy transitions within the atoms. Photons are 2-dimensional confinements of electromagnetic energy and demonstrate the property of inertia (electromagnetic mass) in the 2 directions of confinement. In the $3^{\text {rd }}$ direction, the direction of propagation, photons can only be considered as an electromagnetic wave and for that reason do not demonstrate the property of inertia. All we know about light, and in general about any electromagnetic field configuration, has been based only on two fundamental theories. James Clerk Maxwell introduced in 1865 the "Theory of Electrodynamics" with the publication: "A Dynamical Theory of the Electromagnetic Field" and Albert Einstein introduced in 1905 the "Theory of Special Relativity" with the publication: "On the Electrodynamics of Moving Bodies" and in 1913 the "Theory of General Relativity" with the publication "Outline of a Generalized Theory of Relativity and of a Theory of Gravitation". However, both theories are not capable to explain the property of electromagnetic mass and in specific the anisotropy of the phenomenon of electromagnetic mass. To understand what electromagnetic inertia and the corresponding electromagnetic mass is and how the anisotropy of electromagnetic mass can be explained and how it has to be defined, a New Theory about Light has to be developed. A part of this New Theory about Light will be published in this article.

The New Theory about Light has been based on one single fundamental property of our Universe. The unique property that there has always been, is always and will always be a perfect equilibrium within our Universe. Isaac Newton has discovered this fundamental physical law already 300 years ago by his third law in physics. "For every action there is an equal and opposite reaction". In the New Theory this law of Equilibrium has been extended for any arbitrary Electromagnetic Field Configuration, which requires the fundamental Universal Property: 
"The total algebraic sum of all force densities will always equal zero at any time at any spatial coordinate in any spatial direction".

To develop a set of 4 electromagnetic equations, describing all the force densities within any arbitrary electromagnetic field configuration, the Divergence of the 4-Dimensional Stress-Energy Tensor has been taken, resulting in the 4-Dimensional Electromagnetic Vector Equation with the fundamental requirement: "The the total algebraic sum of all force densities equals zero at any time at any spatial coordinate in any spatial direction" 


\subsection{The Unified 4-Dimensional Hyperspace Equilibrium Equation (Newton's Law in 4 Dimensions):}

The 4-dimensional Electromagnetic "Maxwell Tensor" has been defined by:

$$
\mathrm{F}_{a b}=\partial_{b} \varphi_{a}-\partial_{a} \varphi_{b}
$$

The 4-dimensional Electromagnetic "Energy Momentum Tensor" has been defined by:

$$
T^{a b}=\frac{1}{\mu_{0}}\left[F_{a c} F^{c b}+\frac{1}{4} \delta_{a b} F_{c d} F^{c d}\right]
$$

The 4-dimensional divergence of the Energy Momentum Tensor equals the 4-dimensional Force Density 4-vector:

$$
f^{a}=\partial_{b} \mathrm{~T}^{a b}
$$

The new theory has been based on the fundamental concept of Harmony in which all force densities in the Universe have been counter balanced by equal and opposite directed force densities resulting in a net force density equals zero (Newton's $3^{\text {rd }}$ law) and a final set of 4 Electromagnetic Equations, representing the 4-Dimensional equation of Newton (expressed in force densities) applied to an electromagnetic field, results in:

$$
f^{a}=\partial_{b} \mathrm{~T}^{a b}=0
$$

In the absence of any Gravity, the force density $f^{a}$ in the 3 directions of the 3 coordinates of the chosen 3-coordinate system follows from the (4-dimensional) Divergence of the (4-dimensional) Stress Energy Tensor $(8,9,38)(3)$.

The Divergence of a Vector equals a Scalar. The Divergence of a Tensor equals a Vector. The 4-dimensional Divergence of the 4- 
dimensional Stress Energy Tensor (4) equals the 4-dimensional Forcedensity Vector $f^{a}$.

The first 3 terms of the 4-dimensional Force-density vector equal the force densities in the corresponding 3 dimensions of the chosen Coordinate System. The $4^{\text {th }}$ component equals the Electromagnetic Poynting's Theorem (5) in the time direction $\mathrm{x}_{4}$ (Continuity Equation), which is the $4^{\text {th }}$ term of Newton's equation.

To calculate the equilibrium conditions to present the force densities in the Electromagnetic Field Configuration, the first 3 terms of the 4dimensional Force-density vector are being used. By re-arranging the first 3 terms of the (4-dimensional Divergence) of the (4-dimensional) Stress Energy Tensor (4) results in equation (5) in the spatial directions $\left(\mathrm{x}_{1}, \mathrm{x}_{2}, \mathrm{x}_{3}\right)$ for the 3-dimensional force density $f^{a}$ within the Electromagnetic Field Configuration which is an improved version of Maxwell's 4 equations describing in a classical way the electromagnetic field.

$$
\begin{aligned}
& -\frac{1}{c^{2}} \frac{\partial(\overline{\mathrm{E}} \times \overline{\mathrm{H}})}{\partial t}+\varepsilon_{0} \overline{\mathrm{E}}(\nabla . \overline{\mathrm{E}})-\varepsilon_{0} \overline{\mathrm{E}} \times(\nabla \times \overline{\mathrm{E}})+ \\
& +\mu_{0} \overline{\mathrm{H}}(\nabla . \overline{\mathrm{H}})-\mu_{0} \overline{\mathrm{H}} \times(\nabla \times \overline{\mathrm{H}})=\overline{0}
\end{aligned}
$$

Equation (5) in the 3 spatial directions $\left(\mathrm{x}_{1}, \mathrm{x}_{2}, \mathrm{x}_{3}\right)$ represents the 3dimensional force density $f^{a}$ in a coordinate-free vector equation in the absence of any Gravity, which is the 3 Dimensional representation of Newton's $3^{\text {rd }}$ law expressed in electromagnetic force densities within an arbitrary electromagnetic field configuration and simultaneously represents the improved version of Maxwell's 4 equations.

Equation (5) is a much more powerful equation than the well-known 4 Maxwell equations and offers solutions for many physically well-known electromagnetic field configurations like a LASER beam and the projection of a slide on a screen which cannot be mathematically described by Maxwell's equations. Because LASER beams and projected images can never be divergence free for the electric field and/or the magnetic field intensities at any place at any time.

The scope of Newton's $3^{\text {rd }}$ law (the algebraic sum of all the forces 
equals zero) has been underestimated. Newton's $3^{\text {rd }}$ law expressed in force densities and applied to the electromagnetic force densities within a beam of light results in a much more powerful equation than the wellknown 4 Maxwell equations and also completely includes all the possible solutions of the Maxwell equations.

According Newton's $3^{\text {rd }}$ law, the fundamental requirement of a perfect equilibrium at any place at any time in any direction, the algebraic sum of all the different force densities $\vec{f}$ has to be equal zero for any physical possible electromagnetic field configuration (particles and fields).

3-Dimensional Space Domain

$$
\left(\begin{array}{c}
\mathrm{x}_{3} \\
\mathrm{x}_{2} \\
\mathrm{x}_{1}
\end{array}\right) \quad-\frac{1}{c^{2}} \frac{\partial(\overline{\mathrm{E}} \times \overline{\mathrm{H}})}{\partial t}+\varepsilon_{0} \overline{\mathrm{E}}(\nabla . \overline{\mathrm{E}})-\varepsilon_{0} \overline{\mathrm{E}} \times(\nabla \times \overline{\mathrm{E}})+
$$

It is fundamental to realize that 3 different kind of separate interactions of the force densities in (5) are being involved within this resulting perfect equilibrium. Magnetic-Magnetic interaction (terms B-4 and B-5) , Electric-Electric interaction (terms B-2 and B-3) and Electric-Magnetic interaction (term B-1) which equals the time-derivative of the the Poynting vector and represents the inertia of electromagnetic radiation and is comparable with Newton's second law of motion ( $\mathrm{F}=\mathrm{m} \mathrm{a}=\mathrm{m} \mathrm{d}$ $\mathrm{p} / \mathrm{dt}$ ) for mechanical objects.

The force density vector $f^{a}$ is 4-dimensional. The 4-dimensional Divergence of the 4-dimensional Stress Energy Tensor (4) equals the 4dimensional Force-density Vector $f^{a}$ and can be written as the Electromagnetic "4-Dimensional Hyperspace Equilibrium Equation" (Newton's $3^{\text {rd }}$ Law in 4 Dimensions expressed in electromagnetic force densities): 


\section{Energy-Time Domain}

$\left(\mathrm{x}_{4}\right) \quad \nabla \cdot(\overline{\mathrm{E}} \times \overline{\mathrm{H}})+\frac{1}{2} \frac{\partial\left(\varepsilon_{0}(\overline{\mathrm{E}} \cdot \overline{\mathrm{E}})+\mu_{0}(\overline{\mathrm{H}} \cdot \overline{\mathrm{H}})\right)}{\partial t}=0$

3-Dimensional Space Domain

B-1

B-2

B-3

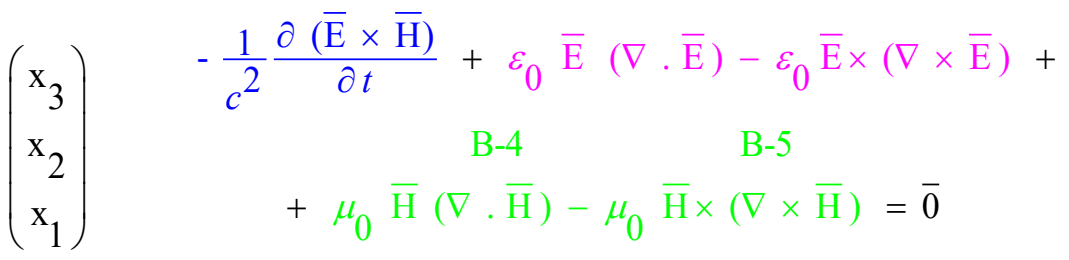

An electromagnetic field which is in a perfect equilibrium with itself and its surrounding at any space and time in any direction, fulfills the necessary requirements for the physical possibility of the existence of this field. Under that condition Equation (4) transforms into the "Unified 4-Dimensional Hyperspace Equilibrium Equation" (7) which is the 4Dimensional representation of Newton's $3^{\text {rd }}$ Law expressed in electromagnetic force densities.

To extend Field Equation (5) into an equilibrium within a multidimensional curved Space-Time continuum unifying different fields like gravity and electromagnetism, the transformation has been realized by the transformation of the resulting force-densities within the 4Dimensional Space-Time continuum. The Unification of the Electromagnetic Fields with the Gravitational fields results in the "Relativistic Gravitational Electromagnetic Equilibrium Equation" (RGEE) within a gravitational field $\vec{g}$ in the 3-dimensional (spatial) representation:

$$
\begin{aligned}
& -\frac{1}{c^{2}} \frac{\partial(\bar{E} \times \bar{H})}{\partial t}+\varepsilon_{0} \bar{E}(\nabla . \bar{E})-\varepsilon_{0} \bar{E} \times(\nabla \times \bar{E})+\mu_{0} \bar{H}(\nabla \cdot \bar{H})- \\
& -\mu_{0} \bar{H} \times(\nabla \times \bar{H})-\frac{1}{2} \varepsilon_{0}^{2} \mu_{0}(\bar{E} \cdot \bar{E}) \overline{\mathrm{g}}-\frac{1}{2} \varepsilon_{0} \mu_{0}^{2}(\bar{H} \cdot \bar{H}) \overline{\mathrm{g}}=\overline{0}
\end{aligned}
$$

It is fundamental to realize that Equation (8) is only a part of the 4Dimensional Time-Space Continuum Equation related to the spatial coordinates.

The 4-Dimensional Divergence of the 4-Dimensional Stress-Energy 
Tensor (2) results in the 4-Dimensional Force Density $f^{a}$ Vector Equation (3). Fulfilling the fundamental law of Universal Equilibrium (Newton's $3^{\text {rd }}$ law) which can be expressed as: "The total algebraic sum of all force densities will always equal zero at any time at any spatial coordinate in any spatial direction" results in the fundamental 4Dimensional Vector Equation (4). The first 3 terms in the 3-Dimensional Space Domain of the 4-Dimensional Vector Equation (7) have been presented in (5) and (6) and represent the improved Maxwell Equations.

The $4^{\text {th }}$ term in the Energy-Time domain, described in (7), presents the Continuum Equation [1],[2]. By introducing the complex field notations for the electric field and the magnetic field in Equation (7), the $4^{\text {th }}$ term transforms [3]-[6] into the well-known relativistic quantum mechanical Dirac Equation [7]-[9] and at low velocities into the quantum mechanical Schrödinger Wave Equation [10].

The Poynting Theorem $\left(4^{\text {th }}\right.$ term in (7)) can be rewritten by introducing the vector functions $\bar{\phi}$ and the complex conjugated vector function $\bar{\phi}^{*}$ in which:

$$
\bar{\phi}=\frac{1}{\sqrt{2 \mu}}\left(\overline{\mathrm{B}}+\mathrm{i} \frac{\overline{\mathrm{E}}}{c}\right)
$$

$\overline{\mathrm{B}}$ equals the magnetic induction, $\overline{\mathrm{E}}$ the electric field intensity and $\mathrm{c}$ the speed of light. The complex conjugated vector function equals:

$$
\overline{\phi^{*}}=\frac{1}{\sqrt{2 \mu}}\left(\overline{\mathrm{B}}-\mathrm{i} \frac{\overline{\mathrm{E}}}{\mathrm{c}}\right)
$$

The dot product equals the electromagnetic energy density w:

$$
\bar{\phi} \cdot \overline{\phi^{*}}=\frac{1}{2 \mu}\left(\overline{\mathrm{B}}+\mathrm{i} \frac{\overline{\mathrm{E}}}{c}\right) \cdot\left(\overline{\mathrm{B}}-\mathrm{i} \frac{\overline{\mathrm{E}}}{c}\right)=\frac{1}{2} \mu \mathrm{H}^{2}+\frac{1}{2} \varepsilon \mathrm{E}^{2}=\mathrm{w}
$$

The cross product is proportional to the Poynting vector [11].

$$
\bar{\phi} \times \overline{\phi^{*}}=\frac{1}{2 \mu}\left(\overline{\mathrm{B}}+\mathrm{i} \frac{\overline{\mathrm{E}}}{c}\right) \times\left(\overline{\mathrm{B}}-\mathrm{i} \frac{\overline{\mathrm{E}}}{c}\right)=\mathrm{i} \sqrt{\varepsilon \mu} \overline{\mathrm{E}} \times \overline{\mathrm{H}}=\mathrm{i} \sqrt{\varepsilon \mu} \overline{\mathrm{S}}
$$

Substituting (11) and (12) in the $4^{\text {th }}$ term (Energy-Time domain) in (7) 
results in the 4-Dimensional Hyperspace Equilibrium Equation (Newton's Law in 4 Dimensions) (13):

$$
\begin{aligned}
& \text { Energy-Time Domain } \\
& \left(\mathrm{x}_{4}\right)-\frac{i}{\sqrt{\varepsilon_{0} \mu_{0}}} \nabla \cdot(\bar{\phi} \times \bar{\phi})=-\frac{\partial \bar{\phi} \cdot \bar{\phi}^{*}}{\partial t} \\
& \text { 3-Dimensional Space Domain } \\
& \left(\begin{array}{c}
\mathrm{x}_{3} \\
\mathrm{x}_{2} \\
\mathrm{x}_{1}
\end{array}\right)^{-\frac{1}{c^{2}} \frac{\partial(\overline{\mathrm{E}} \times \overline{\mathrm{H}})}{\partial t}+\varepsilon_{0} \overline{\mathrm{E}}(\nabla \cdot \overline{\mathrm{E}})-\varepsilon_{0} \overline{\mathrm{E}} \times(\nabla \times \overline{\mathrm{E}})+}+
\end{aligned}
$$

To transform the electromagnetic vector wave function $\bar{\phi}$ into a scalar (spinor or one-dimensional matrix representation), the Pauli spin matrices $\sigma$ and the following matrices [10] have been introduced:

$$
\bar{\alpha}=\left[\begin{array}{ll}
0 & \sigma \\
\sigma & 0
\end{array}\right] \text { and } \bar{\beta}=\left[\begin{array}{cc}
\delta_{a b} & 0 \\
0 & -\delta_{a b}
\end{array}\right]
$$

Then (13) can be rewritten as the 4-Dimensional Hyperspace Equilibrium Dirac Equation (Newton's $3^{\text {rd }}$ Law in 4 Dimensions in a quantum mechanical presentation) (15):

$$
\begin{gathered}
\text { Energy-Time Domain } \\
\left(\mathrm{x}_{4}\right) \quad\left(\frac{\mathrm{i} \mathrm{m} \mathrm{c}}{h} \bar{\beta}+\bar{\alpha} \cdot \nabla\right) \psi=-\frac{1}{c} \frac{\partial \psi}{\partial t} \\
\text { 3-Dimensional Space Domain } \\
\left(\begin{array}{c}
\mathrm{x}_{3} \\
\mathrm{x}_{2} \\
\mathrm{x}_{1}
\end{array}\right) \begin{array}{c}
-\frac{1}{c^{2}} \frac{\partial(\overline{\mathrm{E}} \times \overline{\mathrm{H}})}{\partial t}+\varepsilon_{0} \overline{\mathrm{E}}(\nabla \cdot \overline{\mathrm{E}})-\varepsilon_{0} \overline{\mathrm{E}} \times(\nabla \times \overline{\mathrm{E}})+ \\
+\mu_{0} \overline{\mathrm{H}}(\nabla \cdot \overline{\mathrm{H}})-\mu_{0} \overline{\mathrm{H}} \times(\nabla \times \overline{\mathrm{H}})=\overline{0}
\end{array}
\end{gathered}
$$

The $4^{\text {th }}$ term in the Energy-Time domain, described in (7) represents the relativistic quantum mechanical Dirac Equation (15) [10].

The 4 Equations together (3 Equations for the separate space coordinates) and the Dirac/Schrödinger Equation (for the timecoordinate) describe the Unification in a perfect Equilibrium of the different Fields (Electromagnetic Field, Gravitational Field) 


\subsection{Fundamental Solutions of the Unified 4-Dimensional Hyperspace Equilibrium Equation}

The required Electromagnetic Field Configuration for a perfect Equilibrium in Space and Time for the total algebraic sum of all the force densities being involved (electromagnetic radiation pressure, electromagnetic inertia and electromagnetic interaction) follows from the "Unified 4-Dimensional Hyperspace Equilibrium Equation" (5). 


\subsection{The propagation of a beam of light in one single direction with an arbitrary electromagnetic intensity division in the plane perpendicular to the direction of propagation:}

The first example for the many possible Electromagnetic Field Configuration as a solution of the "Unified 4-Dimensional Hyperspace Equilibrium Equation" represents a beam of light propagating in the zdirection and an arbitrary division $\mathrm{f}(\mathrm{x}, \mathrm{y})$ of intensity in the $(\mathrm{x}, \mathrm{y})$ plane. The solution for the Electric Field Intensity (Ref. https://doi.or/10.31219/osf.io/gbn4p Page 2) in Cartesian Coordinates $\{t, x, y, z\}$ for the Electric Field Component $\mathrm{e}(x, y, z, t)$ equals:

$$
\left(\begin{array}{l}
e_{x} \\
e_{y} \\
e_{z}
\end{array}\right)=\left(f(x, y) g\left(t-\left(\frac{\mathrm{K}_{1}}{z}+1\right) z \sqrt{\varepsilon_{0} \mu_{0}}\right)\right)
$$

The required Electromagnetic Field Configuration for a perfect Equilibrium in Space and Time follows from the Dynamic Equilibrium Equation (5) and equals in Cartesian Coordinates $\{x, y, z, t\}$ for the Magnetic Field Components $\mathrm{m}(t, x, y, z)$ :

$$
\left(\begin{array}{l}
m_{x} \\
m_{y} \\
m_{z}
\end{array}\right)=\sqrt{\frac{\varepsilon_{0}}{\mu_{0}}}\left(f(x, y) g\left(t-\left(\frac{\mathrm{K}_{1}}{z}+1\right) z \sqrt{\varepsilon_{0} \mu_{0}}\right)\right)
$$

In which $K_{1}$ is an arbitrary constant. For the divergence-free function $f(x, y)=1$, the solutions (16) and (17) are also the solutions for the wellknown Maxwell Equations. For the non-divergence-free functions $f(x, y)$, the solutions (16) and (17) are not solutions for the Maxwell Equations, which requires divergence-free electromagnetic waves, propagating with the speed of light $c=1 / \sqrt{\varepsilon_{0} \mu_{0}}$, in the absence of any matter. But they are solutions of the Dynamic Equilibrium Equation (5) and clearly do exist in physics. Comparable with the projection of a slide with a beamer on a flat screen in the z-direction. In which the slide has 
an arbitrary intensity-division $f(x, y)$. The information $f(x, y)$ on the slide propagates with the speed of light $c=1 / \sqrt{\varepsilon_{0} \mu_{0}}$ towards the screen in the $z$-direction in this example while the speed of propagation in the $\mathrm{x}$ - and $\mathrm{y}$-direction equals zero.. 


\subsection{The pre-eminence of the Unified 4-Dimensional Hyperspace Equilibrium Equation compared to the classical 4 Maxwell equations:}

James Clerk Maxwell (1831-1879) was one of the greatest scientists who have ever lived. To him we owe the most significant discovery of our age - the theory of electromagnetism in which theory the electric field and the magnetic field has been unified as one physical phenomenon. He is rightly acclaimed as the father of modern physics. He also made fundamental contributions to mathematics, astronomy and engineering.

Albert Einstein said: "The special theory of relativity owes its origins to Maxwell's equations of the electromagnetic field." Einstein also said: "Since Maxwell's time, physical reality has been thought of as represented by continuous fields, and not capable of any mechanical interpretation [12]-[15]. This change in the conception of reality is the most profound and the most fruitful that physics has experienced since the time of Newton" Einstein called James Clerk Maxwell the greatest physicists of his century.

And indeed, James Clerk Maxwell fully deserves all the credits that has been given to him. But we should not forget Isaac Newton. Because Isaac Newton has discovered the most fundamental law of our universe. The fundamental law of Equilibrium. And on this classical fundamental law of Equilibrium, the "New Theory" has been grounded. And based on that single law of Equilibrium the "New Theory" is able to offer mathematical solutions where Maxwell's 4 equations do fail.

The preeminence of the "Unified 4-Dimensional Hyperspace Equilibrium Equation" compared to the 4 Maxwell equations can be easily demonstrated with the simple example of a LASER beam propagating through space. The intensity division for a LASER beam is not Divergence-Free and for that reason not a solution of the 4 Maxwell Equations. However, the basic example of a LASER beam propagating through space is an exact solution of the "Unified 4-Dimensional Hyperspace Equilibrium Equation" which offers solutions in NonDivergence-Free Electromagnetic Field Configurations.

In general, a LASER beam can be described with a Gaussian intensity division for the electromagnetic field in the plane perpendicular to the direction of propagation. 


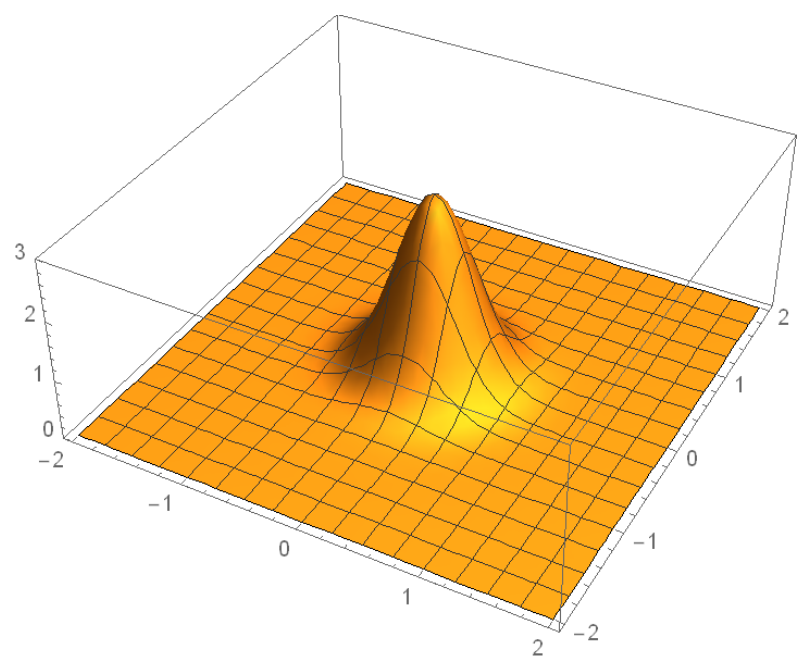

\section{Fig. 1. Electromagnetic Field Intensity with a Gaussian division}

The required Electromagnetic Field Configuration [16], [17], (Ref. https://doi.or/10.31219/osf.io/gbn4p Page 8), which is a mathematical solution of the "Unified 4-Dimensional Hyperspace Equilibrium Equation" (5) equals in Cartesian Coordinates $\{x, y, z, t\}$ for the Electric Field Intensity components $\mathrm{e}(t, x, y, z)$ :

$$
\left(\begin{array}{l}
e_{x} \\
e_{y} \\
e_{z}
\end{array}\right)=\left(\begin{array}{c}
K_{1} \mathrm{e}^{-K_{2}\left(x^{2}+y^{2}\right)} \operatorname{Sin}\left(\omega\left(t-z \sqrt{\varepsilon_{0} \mu_{0}}\right)\right) \\
0 \\
0
\end{array}\right)
$$

The required Electromagnetic Field Configuration [16], [17] for a perfect Equilibrium in Space and Time follows from the Dynamic Equilibrium Equation (5) and equals in Cartesian Coordinates $\{x, y, z, t\}$ for the Magnetic Field Intensity components $\mathrm{m}(t, x, y, z)$ :

$$
\left(\begin{array}{l}
m_{x} \\
m_{y} \\
m_{z}
\end{array}\right)=\sqrt{\frac{\varepsilon_{0}}{\mu_{0}}}\left(\begin{array}{c}
0 \\
K_{1} \mathrm{e}^{-K_{2}\left(x^{2}+y^{2}\right)} \operatorname{Sin}\left(\omega\left(t-z \sqrt{\varepsilon_{0} \mu_{0}}\right)\right) \\
0
\end{array}\right)
$$

In which $K_{1}$ is an arbitrary constant. The Gaussian division cannot be 
a solution of the Maxwell Equations, because the divergence of the Electric field intensity does not equal zero.

$$
\nabla \cdot \overline{\mathrm{E}}=-2 \mathrm{e}^{-\mathrm{K}_{2}\left(\mathrm{x}^{2}+\mathrm{y}^{2}\right)} \mathrm{X} \mathrm{K}_{1} \mathrm{~K}_{2} \operatorname{Sin}\left(\left(t-z \sqrt{\varepsilon_{0} \mu_{0}}\right) \omega\right)(20)
$$

And the Divergence of the Magnetic field intensity does not equal zero.

$$
\nabla . \overline{\mathrm{H}}=-\sqrt{\frac{\varepsilon_{0}}{\mu_{0}}}\left(2 \mathrm{e}^{-\mathrm{K}_{2}\left(\mathrm{x}^{2}+\mathrm{y}^{2}\right)} \mathrm{yK}_{1} \mathrm{~K}_{2} \operatorname{Sin}\left(\left(t-z \sqrt{\varepsilon_{0} \mu_{0}}\right) \omega\right)\right)(21)
$$

For the non-divergence-free functions, the solutions (18) and (19) are not solutions for the Maxwell Equations, which requires divergence-free electromagnetic waves, propagating with the speed of light $c=1 / \sqrt{\varepsilon_{0} \mu_{0}}$ , in the absence of any matter. But they are solutions of the Dynamic Equilibrium Equation (5) and clearly do exist in physics. Since 1960 the LASER beam is a physical reality when the first LASER has been built by Theodore H. Maiman at the Hughes Research Laboratories. A physical phenomenon that could not be explained by Maxwell's equations but is an existing solution of the Dynamic Equilibrium Equation (5). 


\subsection{Electromagnetic Mass (Inertia):}

In a classical way mass (inertia) has been defined by Newton's $2^{\text {nd }}$ law. Einstein already discovered that mass (inertia, m) and energy (W) are related by $\mathrm{W}=\mathrm{m} \mathrm{c}^{2}$. In this chapter a new definition of mass will be represented which is based on the relativistic changes in the radiation pressure of confined electromagnetic radiation during acceleration or deceleration.

\subsection{Confined Electromagnetic Mass (Inertia):}

The definition of mass is related to concept of inertia, defined by Newton's second law. For this reason, free electromagnetic radiation cannot be related to the concept of mass because it is impossible to accelerate or to decelerate free electromagnetic radiation. The speed of light is a universal constant. Only confined electromagnetic radiation can be accelerated or decelerated and for this reason electromagnetic mass (inertia) can only be assigned to confined electromagnetic radiation. 


\subsection{The Origin of Electromagnetic Mass (Inertia):}

Newton described in his second law of motion the classical definition of mass (inertia). However, it is impossible to calculate with Newton's second law of motion the (electromagnetic) mass of a beam of light. Because the speed of light is a universal constant which follows from Albert Einstein's Theory of Special Relativity, it is impossible to accelerate or to slow down a beam of light and for that reason it is impossible to determine the electromagnetic mass of a beam of light (free electromagnetic radiation) by Newton's second law.

To calculate the electromagnetic mass of free or confined electromagnetic radiation, the fundamental concept of the New Theory has been used that the Universe is in a perfect Equilibrium and that any electromagnetic field configuration is in a perfect equilibrium with itself and its surrounding. From this fundamental concept follows a different definition of (confined) electromagnetic mass.

Electromagnetic mass (or inertia) has been determined by the relativistic Lorentz transformation of the radiation pressures in all different directions and the disturbance of a uniform motion (or position at rest) of confined electromagnetic radiation results in a relativistic effect which we measure (experience) as electromagnetic mass (inertia).

The mass in $[\mathrm{kg}]$ of an object will be generally measured by acceleration (or deceleration) of the object according Newton's second law of motion. In the theory of special relativity, the speed of light is a fundamental constant and the intensity of the light is not a universal constant.

The calculate the relativistic mass of Confined Electromagnetic Radiation, we start with a thought experiment in which a beam of light is propagating between two $100 \%$ reflecting mirrors, indicated as Mirror $\mathrm{A}$ and Mirror B. Both mirrors are part of a rigid construction and the relative velocity between both mirrors always equals zero. The results of this calculation will be be generalized for any kind of electromagnetic radiation which has been confined by its own electromagnetic and gravitational field. When the speed of the observer has the same speed as the speed of the light source, then the observer and the light source are relative at rest. And the same light intensity will be measured at the location of the emitter and at the location of the observer.

When the observer moves towards the emitter, the intensity of the light 
at the location of the observer will increase with $\gamma(1+\mathrm{v} / \mathrm{c})$ according the Lorentz transformation in which " $\mathrm{v}$ " is the relative velocity between emitter and observer. At low velocities the term $\gamma$ will equal 1.

When the observer moves away from the emitter, the intensity of the light at the location of the observer will decrease with $\gamma(1-\mathrm{v} / \mathrm{c})$ according the Lorentz transformation. At low velocities the term $\gamma$ will equal 1.

When light is confined between two $100 \%$ reflecting mirrors, then we can conclude that the speed of both mirrors will always be equal, relative to each other. And at uniform speed, the radiation pressures on both mirrors will be equal and both opposite directed radiation pressures will neutralize.

During acceleration, it will take time for the light to travel with the speed of light between both mirrors. When we consider one mirror as the emitter and the opposite mirror as the observer, we can conclude that the speed of the emitter will be different (when the beam of light leaves the emitter) than the speed of the observer (when the beam of light reaches the observer) because of the time interval, needed for the beam of light to propagate from emitter to observer during the acceleration.

During the acceleration, both opposite oriented radiation pressures on both mirrors will not be equal anymore and they will not neutralize each other anymore. During acceleration, there will be a resulting force according Newton's second law of motion caused by both different radiation pressures.

For the first step in this calculation an imaginary experiment has been used. Two $100 \%$ reflecting mirrors B and A (both in the x-y plane and without any mass) are placed opposite each other at a distance $\Delta \mathrm{x}$ [10]. A single harmonic electromagnetic wave has been confined between both mirrors. Between both mirrors a "Standing Electromagnetic Wave" appears which has been formed by two waves travelling in opposite directions along the z-axis.

The Poynting vector corresponding with the electromagnetic wave propagating along the $\mathrm{z}$-axis in the + direction (positive direction of the $\mathrm{z}$-axis) has been indicated as $\overline{\mathrm{S}}^{+}=\overline{\mathrm{E}}^{+} \times \overline{\mathrm{H}}^{+}$and the Poynting vector corresponding with the electromagnetic wave propagating along the $\mathrm{z}$ axis in the - direction (opposite direction) has been indicated as $\bar{S}^{-}=\overline{\mathrm{E}}^{-} \times \overline{\mathrm{H}}^{-}$. The system is at rest. The radiation pressures, caused by 
the confined electromagnetic radiation, on both mirrors $\mathrm{A}$ and $\mathrm{B}$ are opposite and equal in magnitude:

$$
\mathbf{P}_{\mathrm{A}}=\frac{2 \mathrm{~S}_{\mathrm{A}}}{\mathrm{c}}=\frac{2 \mathrm{~S}_{\mathrm{B}}}{\mathrm{c}}=\mathbf{P}_{\mathrm{B}}
$$

Einstein has formulated this very well. "Velocities are always relative" . To calculate the radiation pressure on Mirror A, the velocities, only relative to Mirror A for the waves with the respective Poynting vectors $\bar{S}^{+}=\overline{\mathrm{E}}^{+} \times \overline{\mathrm{H}}^{+}$and $\overline{\mathrm{S}}^{-}=\overline{\mathrm{E}}^{-} \times \overline{\mathrm{H}}^{-}$, have to be calculated.

2.10.1 The radiation pressure on Mirror A, when Mirror A moves with a velocity $\mathrm{v}$ in the direction of the positive $\mathrm{z}$-axis:

When the system of "Two Mirrors B - A" moves in the direction of the positive $\mathrm{z}$-axis, Mirror A moves in the direction of the positive $\mathrm{z}$-axis and the Poynting vector $\bar{S}^{+}=\overline{\mathrm{E}}^{+} \times \overline{\mathrm{H}}^{+}$will decrease according the Lorentz transformation [11].

$$
\bar{S}_{\mathrm{v}}^{+}=\overline{\mathrm{E}}_{\mathrm{v}}^{+} \times \overline{\mathrm{H}}_{\mathrm{v}}^{+}=\gamma^{2}\left(1-\frac{\mathrm{v}}{\mathrm{c}}\right)^{2}\left(\overline{\mathrm{E}}^{+} \times \overline{\mathrm{H}}^{+}\right)
$$

When the system of "Two Mirrors B - A" moves in the direction of the positive z-axis, Mirror A moves in the direction of the positive z-axis the Poynting vector $\bar{S}^{-}=\overline{\mathrm{E}}^{-} \times \overline{\mathrm{H}}^{-}$will increase according the Lorentz transformation [11].

$$
\bar{S}_{\mathrm{v}}^{-}=\overline{\mathrm{E}}_{\mathrm{v}}^{-} \times \overline{\mathbf{H}}_{\mathrm{v}}^{-}=\gamma^{2}\left(1+\frac{\mathrm{v}}{\mathrm{c}}\right)^{2}\left(\overline{\mathrm{E}}^{+} \times \overline{\mathrm{H}}^{+}\right)
$$

The total radiation pressure, caused by the confined electromagnetic radiation, on mirror A equals:

$$
\mathrm{P}_{\mathrm{A}}=\frac{\mathrm{S}_{A}^{+}+\mathrm{S}_{A}^{-}}{\mathrm{c}}=\frac{\gamma^{2}\left(\left(1-\frac{\mathrm{v}}{\mathrm{c}}\right)^{2}+\left(1+\frac{\mathrm{v}}{\mathrm{c}}\right)^{2}\right)\left(\overline{\mathrm{E}}^{+} \times \overline{\mathrm{H}}^{+}\right)}{\mathrm{c}}
$$




\subsection{The radiation pressure on Mirror B when Mirror B moves with a velocity $v$ in the direction of the positive $z$-axis:}

When the system of "Two Mirrors B - A" moves in the direction of the positive $\mathrm{z}$-axis, Mirror B moves in the direction of the positive $\mathrm{z}$-axis and the Poynting vector $\bar{S}^{-}=\overline{\mathrm{E}}^{-} \times \overline{\mathrm{H}}^{-}$will increase according the Lorentz transformation [11].

$$
\bar{S}_{\mathrm{v}}^{-}=\overline{\mathbf{E}}_{\mathrm{v}}^{-} \times \overline{\mathbf{H}}_{\mathrm{v}}^{-}=\gamma^{2}\left(1+\frac{\mathrm{v}}{\mathrm{c}}\right)^{2}\left(\overline{\mathrm{E}}^{+} \times \overline{\mathbf{H}}^{+}\right)
$$

When the system of "Two Mirrors B - A" moves in the direction of the positive z-axis, Mirror A moves in the direction of the positive z-axis the Poynting vector $\overline{\mathrm{S}}^{+}=\overline{\mathrm{E}}^{+} \times \overline{\mathrm{H}}^{+}$will increase according the Lorentz transformation [11].

$$
\bar{S}_{\mathrm{v}}^{+}=\overline{\mathrm{E}}_{\mathrm{v}}^{+} \times \overline{\mathrm{H}}_{\mathrm{v}}^{+}=\gamma^{2}\left(1-\frac{\mathrm{v}}{\mathrm{c}}\right)^{2}\left(\overline{\mathrm{E}}^{+} \times \overline{\mathrm{H}}^{+}\right)
$$

The total radiation pressure, caused by the confined electromagnetic radiation, on mirror B equals [11].

$$
\mathrm{P}_{\mathrm{A}}=\frac{\mathrm{S}_{A}^{+}+\mathrm{S}_{A}^{-}}{\mathrm{c}}=\frac{\gamma^{2}\left(\left(1+\frac{\mathrm{v}}{\mathrm{c}}\right)^{2}+\left(1-\frac{\mathrm{v}}{\mathrm{c}}\right)^{2}\right)\left(\overline{\mathrm{E}}^{+} \times \overline{\mathrm{H}}^{+}\right)}{\mathrm{c}}
$$

$\mathrm{P}_{\mathrm{A}}$ and $\mathrm{P}_{B}$ are still equal in magnitude and both in opposite direction and still cancel each other. The system fulfils Newton's first law of motion. 


\subsection{Newton's second Law of Motion (Inertia) for Confined Electromagnetic Radiation:}

When the system of "Two Mirrors B - A" accelerates, the velocity increases with $\Delta \mathrm{v}$ in a time interval $\Delta \mathrm{t}$. At time $\mathrm{t}$ the radiation pressures on mirror A and mirror B are presented in [10]) and [11]. At time $\mathbf{t}+\Delta \mathrm{t}$ the radiation pressures on Mirror $\mathrm{A}$ and Mirror $\mathrm{B}$ will different:

The radiation pressure at time $t+\Delta t$ caused by the confined electromagnetic radiation, on mirror A equals [11]:

$\mathrm{P}_{\mathrm{A}}=\frac{\mathrm{S}_{A}^{+}+\mathrm{S}_{A}^{-}}{\mathrm{c}}=\frac{\gamma^{2}\left(\left(1+\frac{(\mathrm{v})}{\mathrm{c}}\right)^{2}+\left(1-\frac{(\mathrm{v}+\Delta \mathrm{v})}{\mathrm{c}}\right)^{2}\right)\left(\overline{\mathrm{E}}^{+} \times \overline{\mathrm{H}}^{+}\right)}{\mathrm{c}}$

Because the wave with Poynting vector $\overline{\mathrm{S}}^{+}=\overline{\mathrm{E}}^{+} \times \overline{\mathrm{H}}^{+}$has left Mirror $B$ at " $\mathrm{t}$ " and during the time interval $\Delta \mathrm{t}$ the magnitude of $\overline{\mathrm{E}}_{\mathrm{t}}^{+}=\left(1+\frac{\mathrm{v}}{\mathrm{c}}\right) \overline{\mathrm{E}}^{+}$ and $\overline{\mathrm{H}}_{\mathrm{t}}^{+}=\left(1+\frac{\mathrm{v}}{\mathrm{c}}\right) \overline{\mathrm{H}}^{+}$has not changed.

The radiation pressure at time $t+\Delta t$ caused by the confined electromagnetic radiation, on mirror $B$ equals [11]:

$\mathrm{P}_{B}=\frac{\mathrm{S}_{B}^{+}+\mathrm{S}_{B}^{-}}{\mathrm{c}}=\frac{\gamma^{2}\left(\left(1+\frac{(\mathrm{v}+\Delta \mathrm{v})}{\mathrm{c}}\right)^{2}+\left(1-\frac{(\mathrm{v})}{\mathrm{c}}\right)^{2}\right)\left(\overline{\mathrm{E}}^{+} \times \overline{\mathrm{H}}^{+}\right)}{\mathrm{c}}$

Because the wave with Poynting vector $\bar{S}^{-}=\overline{\mathrm{E}}^{-} \times \overline{\mathrm{H}}^{-}$has left Mirror A at " $\mathrm{t}$ " and during the time interval $\Delta \mathrm{t}$ the magnitude of $\overline{\mathrm{E}}_{\mathrm{t}}^{-}=\left(1+\frac{\mathrm{v}}{\mathrm{c}}\right) \overline{\mathrm{E}}^{-}$ and $\overline{\mathrm{H}}_{\mathrm{t}}=\left(1+\frac{\mathrm{v}}{\mathrm{c}}\right) \overline{\mathrm{H}}^{-}$has not changed.

The radiation pressures on Mirror A and Mirror B do not counterbalance each other anymore and the resulting radiation pressure equals [11]:

$\mathrm{P}_{\mathrm{B}}-\mathrm{P}_{\mathrm{A}}=\frac{\gamma^{2}(4 \Delta \mathrm{v}) \mathrm{S}}{\mathrm{c}^{2}}$ 
Equation (31) can be written as [11]:

$\mathrm{P}_{\mathrm{B}}-\mathrm{P}_{\mathrm{A}}=\frac{\gamma^{2}(4 \Delta \mathrm{v}) \mathrm{S}}{\mathrm{c}^{2}}=\frac{\gamma^{2}\left(4 \frac{\Delta \mathrm{v}}{\Delta \mathrm{t}}\right) \mathrm{S} \Delta \mathrm{t}}{\mathrm{c}^{2}}=\gamma^{2} \frac{\mathrm{W}}{\mathrm{c}^{2}} \mathrm{a}=\gamma^{2} \mathrm{ma}$

In which the acceleration $a=\frac{\Delta v}{\Delta t}$ and the inertia $m=\frac{W}{c^{2}}$. At nonrelativistic velocities $\gamma=1$ and (22) turns into the Newton's second law of motion. From (22) also Einstein's famous equation $\mathrm{W}=\mathrm{m} \mathrm{c}^{2}$ follows. In (32) $\mathrm{W}$ is the total confined electromagnetic mass.

Now we can consider electromagnetic confinements without the use of mirrors but electromagnetic confinements through electromagnetic gravitational interaction. By superposition and integration over arbitrary surfaces it is possible to prove that all confined electromagnetic radiation equals (32) and represents electromagnetic mass and follows Newton's second law of motion $(\mathrm{F}=\mathrm{m}$ a). 


\subsection{Longitudinal Mass. The Real Light Intensity of the Sun, measured in our Solar: System, including Electromagnetic Gravitational Conversion (EMGC)}

When a beam of light leaves the surface of the sun, the light will travel in the radial direction of the radial gravitational field caused by the sun. This experiment is comparable with throwing up a ball in the direction of the gravitational field on earth. During the rising in height, the kinetic energy of the ball will be transformed into potential energy. The speed of the ball slows down, becomes zero, and during the fall the kinetic potential energy will be transformed again into kinetic energy and the speed of the ball increase again.

Something comparable happens with a beam of light leaving the sun in the direction of the (radial) gravitational field of the sun. But because the speed of light is a universal constant, not the speed of light will be transferred into potential energy, but the electromagnetic energy of the beam of light will be transformed into potential energy. During the time the beam of light leaves the sun, the intensity of the beam of light will become less and the potential energy will increase with the same amount. When a beam of light propagates towards the sun, the electromagnetic field intensity will increase while the potential energy decreases with the same amount.

The required Electromagnetic Field Configuration for a perfect Equilibrium in Space and Time for a Radial Gravitational Field (The Light propagates in the same radial-direction as the radial-direction of the Gravitational Field ) follows from the Gravitational Dynamic Equilibrium Equation (8) and equals in Spherical Coordinates $\{r, \theta, \varphi, t\}$ for a gravitational field " $\mathrm{g}(\mathrm{r})$ " for the Electric Field Components $\mathrm{e}$ $(r, \theta, \varphi, t)$ :

$$
\left(\begin{array}{c}
e_{r} \\
e_{\theta} \\
e_{\varphi}
\end{array}\right)=\left(\begin{array}{cc}
\frac{1}{r} \mathrm{e}^{-1+\frac{\mathrm{G} \mathrm{m}_{1} \varepsilon_{0} \mu_{0}}{2 \mathrm{r}}} & f[\theta, \varphi] \mathrm{h}[t-r \sqrt{\epsilon 0} \sqrt{\mu 0}] \\
0 \\
0
\end{array}\right)
$$

In which $G$ represents the gravitational field intensity $\left[\mathrm{m} / \mathrm{s}^{2}\right]$ of the sun. The required Electromagnetic Field Configuration for a perfect 
Equilibrium in Space and Time for a Radial Gravitational Field (The Light propagates in the same radial-direction as the radial-direction of the Gravitational Field ) follows from the Gravitational Dynamic Equilibrium Equation (8) and equals in Spherical Coordinates $\{r, \theta, \varphi, t\}$ for a gravitational field " $\mathrm{g}(\mathrm{r})$ " for the Magnetic Field Components $\mathrm{m}$ $(r, \theta, \varphi, t)$ :

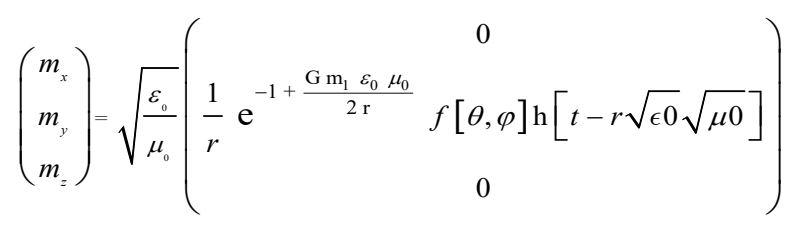

Equation (33) and (34) are solutions of (8) under the influence of a Radial Gravitational field with field a gravitational constant $\mathrm{G}$ and a gravitational field intensity $\mathrm{g}\left[\mathrm{m} / \mathrm{s}^{2}\right]$ :

$$
g=\mathrm{G} \frac{\mathrm{m}_{1}}{\mathrm{r}^{2}}
$$

Which acts along the radial-direction while the electromagnetic wave is also propagating in the radial-direction.

When a light beam leaves the surface of the sun, the intensity will decrease (Ref. https://doi.or/10.31219/osf.io/gbn4p Page 16) according (8). At earth, the measured intensity [11] will be according (8):

$$
\mathrm{I}=\frac{\mathrm{I}_{0} \mathrm{e}^{-2+\frac{\mathrm{Gm} \mathrm{m}_{1} \varepsilon_{0} \mu_{0}}{\mathrm{r}}}}{4 \pi \mathrm{r}^{2}}
$$

A beam of light represents an amount of electromagnetic energy. Which equals an amount of electromagnetic mass. This amount of electromagnetic mass is moving with the speed of light in the opposite direction of a (radial) gravitational field and gains potential energy. Because the law of conservation of energy, a part of the electromagnetic energy of the light beam has to be converted into potential energy according (33) and (34). Which has been presented in Fig. 2, in which the Electromagnetic Gravitational Conversion term has been plotted as a 
function of the distance to the source of the gravitational field.

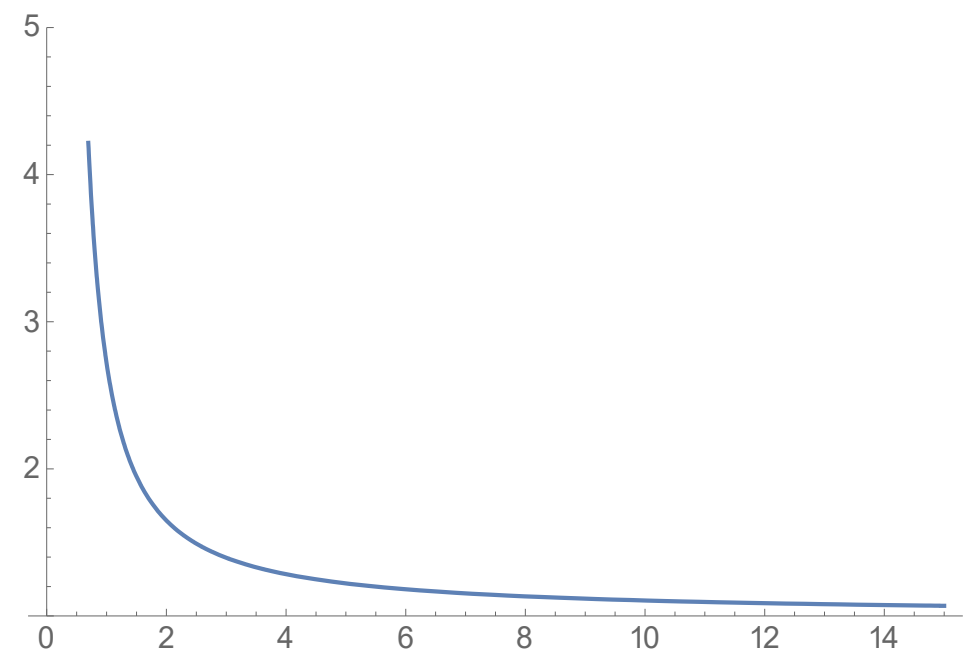

Fig. 2. Electromagnetic Gravitational Conversion Term

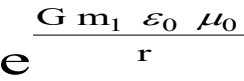

For a radius of the sun equals $695,508[\mathrm{~km}]$ and a distance from the sun to the earth of $149,600,000$ [km], the Electromagnetic Gravitational Conversion (EMGC) term equals:

$$
C_{E M G C}=\frac{\mathrm{e}^{\frac{\mathrm{G} \mathrm{m}_{1} \varepsilon_{0} \mu_{0}}{\mathrm{r}_{1}}}}{\mathrm{e}^{\frac{\mathrm{Gm} \varepsilon_{0} \mu_{0}}{\mathrm{r} 2}}}=\frac{\mathrm{e}^{\frac{1}{\mathrm{r}_{1}}}}{\mathrm{e}^{\frac{1}{\mathrm{r} 2}}}=4.1877534
$$

This means that the real intensity of the light at the surface of the sun is about 4 times higher than the intensity which would have been calculated in a classical way from the sunlight intensity measured on earth, due to Electromagnetic Gravitational Conversion.

Equations (33) and (34) are solutions of (8) under the influence of a Radial Gravitational field with field intensity "G" that acts along the radial-direction while the electromagnetic wave is also propagating in the same radial-direction. The electromagnetic wave is propagating with the unaltered speed of light $c=1 / \sqrt{\varepsilon_{0} \mu_{0}}$, independently of the strength $\mathrm{G}$ of the gravitational field in the radial-direction. However, the amplitude of the electromagnetic wave becomes dependently of the gravitational intensity " $G$ " and the distance " $r$ " and changes along the 
radial direction due to the Electromagnetic-Gravitational Conversion term $C_{E M G C}=\mathrm{e}^{\frac{\mathrm{Gm}_{1} \varepsilon_{0} \mu_{0}}{\mathrm{r}}}$.

Because of the law of conservation of Energy, the electromagnetic energy of the light emitted by the sun is decreasing over a distance " $\mathrm{r}$ " proportional with the same amount $\mathrm{EmGC}=\mathrm{e}^{\frac{\mathrm{Gm} \mathrm{m}_{1} \varepsilon_{0} \mu_{0}}{\mathrm{r}}}$ as the potential energy of the electromagnetic mass of the light emitted by the sun is increasing. 


\subsection{Transversal Electromagnetic Mass (Inertia) / Confined Mass (The Impact of Gravity on Light)}

In chapter 4.1.4 it has already been demonstrated that the classical concept of mass has been related to confined electromagnetic radiation. A LASER beam is an example of 2-Dimensional confinement.

It has already been demonstrated in chapter 4.2 that a LASER beam has no classical mass in the direction of propagation. In the direction of propagation an energy exchange takes place between potential energy (caused by a gravitational field in the direction of propagation) and the electromagnetic energy of the beam itself.

A Laser beam only expresses the classical property of mass in the direction transversal to the direction of propagation. For that reason, a LASER beam propagates around a source of a gravitational field like a satellite moves in a circular orbit around the earth.

We consider a beam of light approaching a strong gravitational field. (E.g. a Black Hole). According the first term in (8) the beam of light will follow a circular orbit around the Black Hole. The required Equilibrium will exist at the radius where the centrifugal electromagnetic inertia forces will be equal and opposite directed to the centripetal oriented gravitational forces on the electromagnetic mass. Figure 3 represents the orbit (colored red) of a LASER beam around a uniform intense gravitational field (Black Hole)

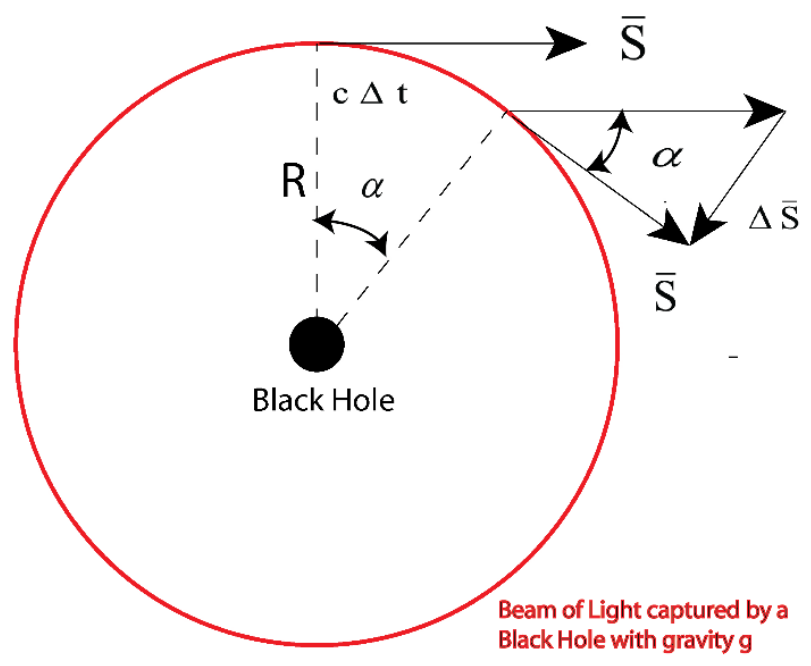

Fig. 3. LASER beam around a Black Hole 
In Figure 3 "the term $\Delta \mathrm{S}$ divided by a time interval $\Delta \mathrm{t}$ " equals:

$$
\frac{\Delta \mathrm{S}}{\Delta \mathrm{t}}=\mathrm{c} \frac{\Delta \mathrm{p}}{\Delta \mathrm{t}}=\mathrm{c} \frac{\Delta(\mathrm{m} \mathrm{v})}{\Delta \mathrm{t}}=\mathrm{c} \mathrm{m} \frac{\Delta \mathrm{v}}{\Delta \mathrm{t}}=\mathrm{c} \mathrm{m} \mathrm{a}
$$

In which "a" represents the radial acceleration and is part of Newton's second law of motion in the radial direction. Because the beam of light has been confined in the radial direction, it demonstrates in the radial direction the property of inertia (electromagnetic mass) and interacts with a gravitational field according Newton's second law of motion. The whole Universe is in a perfect Equilibrium. This fundamental property of Equilibrium has been extended to a 4-dimensional Hyperspace Continuum in which a perfect equilibrium persists in any of the 4 coordinate directions.

The requirement of a 4-dimensional Equilibrium [1]-[4] results in the outcome that the Dirac Equation is only one equation in a set of 4 equations. And that the Dirac Equation originates from an electromagnetic equation in the time-energy domain. This new 4Dimensional Hyperspace Equilibrium Theory opens a new door to an unexplored field of mathematical and physical challenges. This theory is a new approach in physics based on a 4-Dimensional Hyperspace Equilibrium resulting in the 4-dimensional Dirac Equation which represents the "Unification of (quantum mechanical) De Broglie Waves and Electromagnetic Waves". Solving these 4 simultaneous equations (7) offers the possibility to find answers to the fundamental questions in physics within a quantum mechanical 4-Dimensional Frame-Work. Every Physical Possible Electro-Magnetic Field Configuration of Confinement has to be a solution of this fundamental 4-Dimensional Relativistic Dirac Equation (7).

Equation (7) in the Time-Energy Domain (x4) equals the well-known Quantum Mechanical Dirac Equation and in a comparable way the Schrödinger Wave Equation [10] at non-relativistic velocities.

The 3 Equations (7) in the 3 spatial directions (x1, x2, x3) describe the force densities within the Electromagnetic Field. The term B-1 controls the speed of light and is comparable with Newton's second law of motion. When all the external forces acting on confined electromagnetic radiation equal zero, term B-1 represents Newton's $1^{\text {st }}$ law. The terms 
B-2 and B-3 control the confinement of Light for the Electric Radiation Pressure and the terms B-4 and B-5 control the confinement of Light for the Magnetic Radiation Pressure. Equation (7) represents Newton's $3^{\text {rd }}$ law expressed in electromagnetic force densities within an arbitrary electromagnetic field configuration (confined or free electromagnetic radiation at any arbitrary frequency. Equation (7) in the 3 spatial directions is an improved version of the well-known Maxwell's 4 equations. 


\subsection{The Physical properties (inertia, mass, momentum, velocity) of the Photon.}

Photons are unique confinements of Electromagnetic Radiation. They are 2-dimensional confinements of electromagnetic wave packages. The theory describes this completely. Photons can never be accelerated or decelerated (in the direction of propagation) because that is only possible with confined radiation. It is possible to accelerate or decelerate photons in the direction perpendicular to the direction of propagation (directions of confinement) but it is impossible to accelerate or decelerate photons in the direction of propagation. They will always (according the theory) propagate with the speed of light and for that reason a red shift will only be possible when the gravitational field is in a direction perpendicular to the direction of propagation.

As a demonstration of the power of the 4-dimensional Relativistic Dirac Equation, we observe a very basic and simple polarized laser beam with a Gaussian Intensity Division. The laser beam propagates towards the $\mathrm{z}$-direction. The Electric Field " $\mathrm{E}$ " is oriented along the $\mathrm{x}$-axis and the Magnetic Field " $\mathrm{H}$ " is oriented along the y-axis which has been presented in Figure 4.

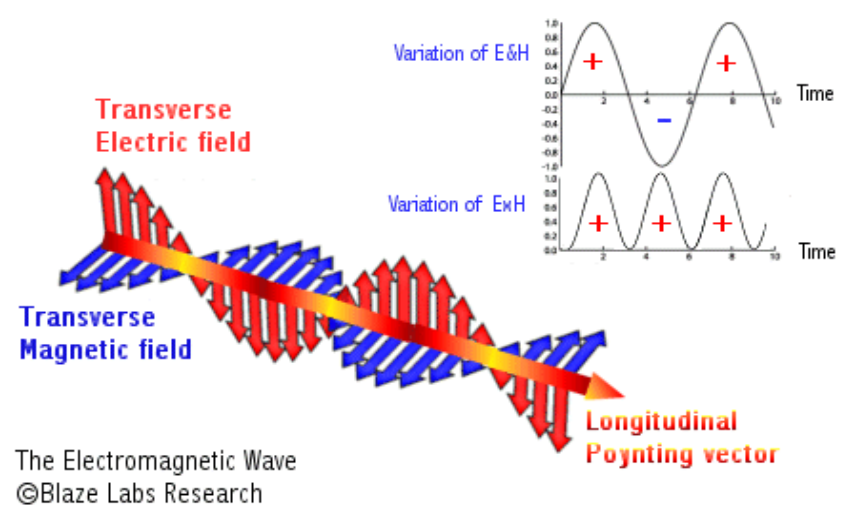

\section{Fig. 4 Polarized LASER beam}

The laser beam propagates towards the positive z-direction. Figure 5 represents a single shot LASER beam where the front of the LASER beam is visible. 


\section{Fig. 5 Polarized LASER beam (Single Shot)}

The laser beam propagates towards the positive z-direction with the speed of light. That is only possible because exactly at the speed of light there will exist a "Perfect Equilibrium" between the Electromagnetic Radiation Pressure towards the positive z-direction and the inertia term of the Electromagnetic Radiation Energy (electromagnetic mass) which has been represented by the time derivative of the "Poynting Vector" in term B-1 and is oriented along the negative z-direction. Figure 6 represents the front of the Single Shot LASER beam in which a perfect equilibrium does exist between the forward oriented radiation pressure $-\nabla \mathrm{w}$ and the inertia of the electromagnetic energy $-\frac{1}{c^{2}} \frac{\Delta \overline{\mathrm{E}} \times \overline{\mathrm{H}}}{\Delta \mathrm{t}}$.

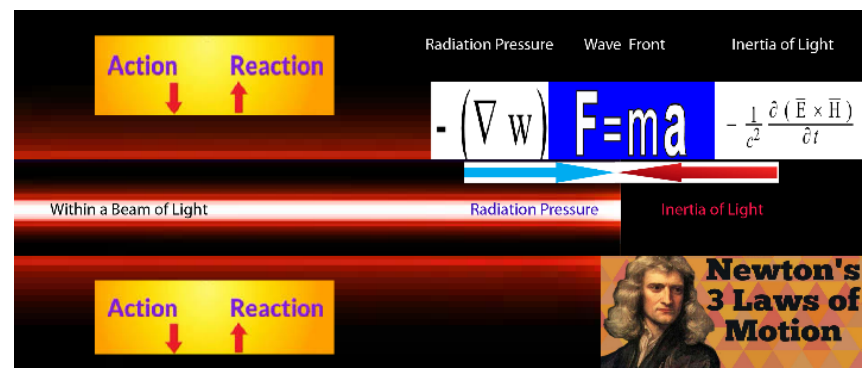

Fig. 6. Newton's second Law of motion applied to a LASER Beam.

In the Unified 4-dimensional Equilibrium always a "Perfect Equilibrium" will exist between the Electromagnetic Radiation Pressure towards the positive z-direction and the inertia term of the Electromagnetic Radiation Energy (electromagnetic mass) which has been represented by the time derivative of the "Poynting Vector" in term B-1 and is oriented along the negative z-direction.

The inward oriented Electromagnetic Force Density in the z-direction (Electromagnetic Inertia) for every arbitrary function $\mathrm{f}(\mathrm{x}, \mathrm{y}, \mathrm{z})$ propagating with the speed of light $\mathrm{c}=1 / \sqrt{\varepsilon_{0} \mu_{0}}$ :

$$
\begin{aligned}
& \mathrm{f}_{z}=-\varepsilon_{0} \mu_{0} \frac{\partial \overline{\mathrm{E}} \times \overline{\mathrm{H}}}{\partial \mathrm{t}} \\
& =-2 \varepsilon_{0}^{3 / 2} \sqrt{\mu_{0}} \mathrm{f}(\mathrm{x}, \mathrm{y})^{2} \mathrm{~g}\left(\mathrm{t}-\mathrm{z} \sqrt{\varepsilon_{0} \mu_{0}}\right) \mathrm{g}\left(\mathrm{t}-\mathrm{z} \sqrt{\varepsilon_{0} \mu_{0}}\right)
\end{aligned}
$$


Compensates the outward oriented Electromagnetic Radiation Pressure in the $\mathrm{z}$-direction for every arbitrary function $\mathrm{f}(\mathrm{x}, \mathrm{y}, \mathrm{z})$ propagating with the speed of light $\mathrm{c}=1 / \sqrt{\varepsilon_{0} \mu_{0}}$ :

$$
\begin{aligned}
& \mathrm{f}_{z}=-(\nabla \mathrm{w})_{z}= \\
& 2 \varepsilon_{0}^{3 / 2} \sqrt{\mu_{0}} \mathrm{f}(\mathrm{x}, \mathrm{y})^{2} \mathrm{~g}\left(\mathrm{t}-\mathrm{z} \sqrt{\varepsilon_{0} \mu_{0}}\right) \mathrm{g}^{\prime}\left(\mathrm{t}-\mathrm{z} \sqrt{\varepsilon_{0} \mu_{0}}\right)
\end{aligned}
$$

The electromagnetic "Radiation Pressure" in the positive z-direction (direction of propagation) has been determined by the electromagnetic "Energy Density". The electromagnetic "Inertia Force" has been determined by the electromagnetic "Mass (Energy Density)" and the "Propagation Speed". There is only "One Exact Single Velocity" at which "Velocity" the electromagnetic "Radiation Pressure" in the in the positive z-direction perfectly counterbalances the opposite directed (negative z-direction) "Force of Inertia". That single speed has been called the "Velocity of Perfect Equilibrium" and has been called in general the "Speed of Light" and is independent of the frequency and the intensity of the electromagnetic beam of light.

Velocity of Perfect Equilibrium equals:

$$
\mathrm{c}=\frac{1}{\sqrt{\varepsilon_{0} \mu_{o}}}=299,792[\mathrm{~km} / \mathrm{s}]
$$

When a laser beam (beam of light) passes a gravitational field with acceleration " $\mathrm{g}$ " in the $(\mathrm{x}, \mathrm{y})$ direction $[5-8]$, the radiation pressures within de the beam of light in the $(x, y)$ plane will shift due to gravitational forces. Because according Einstein's $\mathrm{E}=\mathrm{mc}^{2}$, the electromagnetic energy of the beam of light has electromagnetic mass, which will be attracted by the gravitational field. The shift in the radiation pressures in the $x-y$ plane due to gravitational-electromagnetic interaction can only be compensated by the inertia forces within the $(\mathrm{x}, \mathrm{y})$ plane due to a circular orbit of the beam of light with the origin of the gravitational field as the center. 


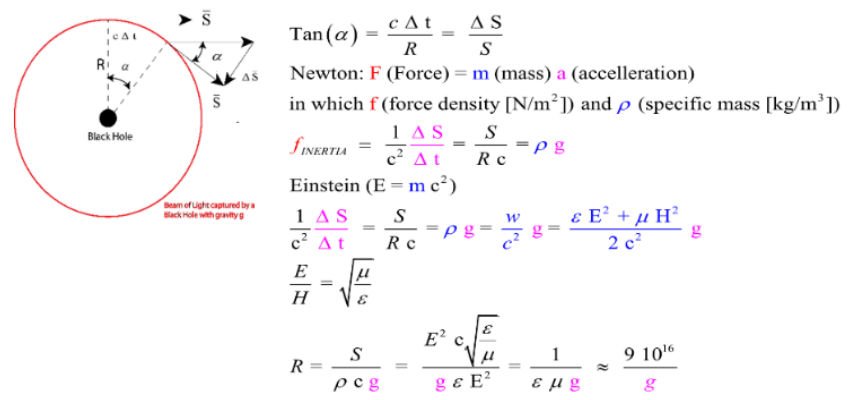

Fig. 7 Polarized LASER beam around a Black Hole

Figure 7 represents the perfect equilibrium between the inertia force densities of the electromagnetic mass $\frac{1}{c^{2}} \frac{\Delta \overline{\mathrm{S}}}{\Delta \mathrm{t}}$ and the centripetal oriented gravitational force density $\frac{\mathrm{w}}{\mathrm{c}^{2}} \overline{\mathrm{g}}$ acting on the electromagnetic mass. The perfect equilibrium direction $[9,10,12,13]$ where the inertia forces due to the circular orbit of the beam of light are in a perfect balance with the attractive gravitational forces, exists at one defined radius " $R$ " of the beam of light (LASER beam), independent of the intensity of the beam of light and independent of the frequency of the beam of light. Only the acceleration " $\mathrm{g}$ " of the gravitational field determines the radius of equilibrium "R"

$\mathrm{R} \approx \frac{910^{16}}{g}$

In which " $\mathrm{R}$ " is the radius of the beam of light and " $\mathrm{g}$ " the acceleration of the gravitational field of the "Black Hole".

The $\mathrm{x}$-y plane is oriented perpendicular on the z-direction. The speed of light towards the positive z-direction equals the speed of light (the constant "c $=300.000 \mathrm{~km} / \mathrm{s}$ "). But the speed of light in the $\mathrm{x}-\mathrm{y}$ plane has to be exactly zero $[9,14,15]$. Else the diameter of the laser beam would become larger and larger during the propagation along the positive $\mathrm{z}$ direction. This is only possible because the Electromagnetic confining forces B-2, B-3, B-4 and B-5 compensate exactly the outward oriented radiation pressure towards the $\mathrm{x}$-direction and the $\mathrm{y}$-direction.

The Electric Radiation Pressure has been compensated by the Coulomb Force Densities within the Laser Beam 
Coulomb's Law (B-2):

$\overline{\mathrm{F}}=\overline{\mathrm{E}} \mathrm{Q} \quad(\mathrm{F} \triangleq$ Force and $\mathrm{Q} \triangleq$ Electric Charge $)$

$\overline{\mathrm{f}}=\overline{\mathrm{E}} \rho \quad(\mathrm{f} \hat{=}$ Force density and $\rho \hat{\triangleq}$ Charge Density)

$\overline{\mathrm{f}}=\overline{\mathrm{E}} \nabla \cdot \overline{\mathrm{D}} \quad(\mathrm{D} \triangleq$ Dielectric Displacement $)$

$\overline{\mathrm{f}}=\varepsilon_{0} \overline{\mathrm{E}} \nabla \cdot \overline{\mathrm{E}}\left(\varepsilon_{0} \triangleq\right.$ Permittivity $)$

$$
\begin{gathered}
\text { Energy-Time Domain } \\
\left(\mathrm{x}_{4}\right)\left(\frac{\mathrm{i} \mathrm{m} \mathrm{c}}{h} \bar{\beta}+\bar{\alpha} \cdot \nabla\right) \psi+\frac{1}{c} \frac{\partial \psi}{\partial t}=0 \\
\text { 3-Dimensional Space Domain } \\
\text { B-1 B-2 } \\
\left(\begin{array}{l}
\mathrm{x}_{3} \\
\mathrm{x}_{2} \\
\mathrm{x}_{1}
\end{array}\right) \quad \begin{array}{c}
\frac{1}{c^{2}} \frac{\partial(\overline{\mathrm{E}} \times \overline{\mathrm{H}})}{\partial t}+\varepsilon_{0} \overline{\mathrm{E}}(\nabla \cdot \overline{\mathrm{E}})-\varepsilon_{0} \overline{\mathrm{E}} \times(\nabla \times \overline{\mathrm{E}})+ \\
\mathrm{B}-4 \\
+\mu_{0} \overline{\mathrm{H}}(\nabla \cdot \overline{\mathrm{H}})-\mu_{0} \overline{\mathrm{H}} \times(\nabla \times \overline{\mathrm{H}})=\overline{0}
\end{array}
\end{gathered}
$$

The Electric Radiation Pressure along the $\mathrm{x}$-axis has been compensated by the term B-2:

The inward oriented Electric Force Density in the x- direction for every arbitrary function $\mathrm{f}(\mathrm{x}, \mathrm{y}, \mathrm{z})$ :

$$
\begin{aligned}
\mathrm{f}_{x} & =\varepsilon_{0} \mathrm{E}_{x} \frac{\partial \mathrm{E}_{x}}{\partial \mathrm{x}} \\
& =\varepsilon_{0} \mathrm{f}(\mathrm{x}, \mathrm{y}) \mathrm{g}\left(\mathrm{t}-\mathrm{z} \sqrt{\varepsilon_{0} \mu_{0}}\right)^{2} \mathrm{f}^{(1,0)}(\mathrm{x}, \mathrm{y})
\end{aligned}
$$

Compensates the outward oriented Electric Radiation Pressure in the $\mathrm{x}$-direction for every arbitrary function $\mathrm{f}(\mathrm{x}, \mathrm{y}, \mathrm{z})$ :

$$
\begin{aligned}
\mathrm{f}_{x} & =-(\nabla \mathrm{w})_{x} \\
& =-\varepsilon_{0} \mathrm{f}(\mathrm{x}, \mathrm{y}) \mathrm{g}\left(\mathrm{t}-\mathrm{z} \sqrt{\varepsilon_{0} \mu_{0}}\right)^{2} \mathrm{f}^{(1,0)}(\mathrm{x}, \mathrm{y})
\end{aligned}
$$

The Electric Radiation Pressure along the y-axis has been compensated by the term B-3:

The inward oriented Electric Force Density in the y- direction for 
every arbitrary function $\mathrm{f}(\mathrm{x}, \mathrm{y}, \mathrm{z})$ :

$$
\begin{aligned}
& \mathrm{f}_{y}=-\varepsilon_{0}(\overline{\mathrm{E}} \times(\nabla \times \overline{\mathrm{E}}))_{y} \\
& =-\varepsilon_{0} \mathrm{f}(\mathrm{x}, \mathrm{y}) \mathrm{g}\left(\mathrm{t}-\mathrm{z} \sqrt{\varepsilon_{0} \mu_{0}}\right)^{2} \mathrm{f}^{(0,1)}(\mathrm{x}, \mathrm{y})
\end{aligned}
$$

Compensates the outward oriented Electric Radiation Pressure in the $\mathrm{y}$-direction for every arbitrary function $\mathrm{f}(\mathrm{x}, \mathrm{y}, \mathrm{z})$ :

$$
\begin{aligned}
\mathrm{f}_{y} & =-(\nabla \mathrm{w})_{y} \\
& =\varepsilon_{0} \mathrm{f}(\mathrm{x}, \mathrm{y}) \mathrm{g}\left(\mathrm{t}-\mathrm{z} \sqrt{\varepsilon_{0} \mu_{0}}\right)^{2} \mathrm{f}^{(0,1)}(\mathrm{x}, \mathrm{y})
\end{aligned}
$$

This also is valid for a single photon. In the directions perpendicular to the direction of propagation, the electric radiation pressures (45) and (47) have been compensated completely by the electric interaction forces (44) and (46)

The Magnetic Radiation Pressure along the $y$-axis has been compensated by the term B-4:

The inward oriented Magnetic Force Density in the y-direction for every arbitrary function $\mathrm{f}(\mathrm{x}, \mathrm{y}, \mathrm{z})$ :

$$
\begin{aligned}
\mathrm{f}_{y} & =\mu_{0} \mathrm{H}_{y} \frac{\partial \mathrm{H}_{y}}{\partial \mathrm{y}} \\
& =\mu_{0} \mathrm{f}(\mathrm{x}, \mathrm{y}) \mathrm{g}\left(\mathrm{t}-\mathrm{z} \sqrt{\varepsilon_{0} \mu_{0}}\right)^{2} \mathrm{f}^{(0,1)}(\mathrm{x}, \mathrm{y})
\end{aligned}
$$

Compensates the outward oriented Magnetic Radiation Pressure in the $y$-direction for every arbitrary function $f(x, y, z)$ :

$$
\begin{aligned}
\mathrm{f}_{y} & =-(\nabla \mathrm{w})_{y} \\
& =-\mu_{0} \mathrm{f}(\mathrm{x}, \mathrm{y}) \mathrm{g}\left(\mathrm{t}-\mathrm{z} \sqrt{\varepsilon_{0} \mu_{0}}\right)^{2} \mathrm{f}^{(0,1)}(\mathrm{x}, \mathrm{y})
\end{aligned}
$$

The Magnetic Radiation Pressure along the $\mathrm{x}$-axis has been compensated by the term B-5:

The inward oriented Magnetic Force Density in the $\mathrm{x}$ - direction for every arbitrary function $\mathrm{f}(\mathrm{x}, \mathrm{y}, \mathrm{z})$ : 


$$
\begin{aligned}
\mathrm{f}_{x} & =-\mu_{0}(\overline{\mathrm{H}} \times(\nabla \times \overline{\mathrm{H}}))_{x} \\
& =-\mu_{0} \mathrm{f}(\mathrm{x}, \mathrm{y}) \mathrm{g}\left(\mathrm{t}-\mathrm{z} \sqrt{\varepsilon_{0} \mu_{0}}\right)^{2} \mathrm{f}^{(1,0)}(\mathrm{x}, \mathrm{y})
\end{aligned}
$$

Compensates the outward oriented Magnetic Radiation Pressure in the $\mathrm{x}$-direction for every arbitrary function $\mathrm{f}(\mathrm{x}, \mathrm{y}, \mathrm{z})$ :

$$
\begin{aligned}
\mathrm{f}_{x} & =-(\nabla \mathrm{w})_{x} \\
& =\mu_{0} \mathrm{f}(\mathrm{x}, \mathrm{y}) \mathrm{g}\left(\mathrm{t}-\mathrm{z} \sqrt{\varepsilon_{0} \mu_{0}}\right)^{2} \mathrm{f}^{(1,0)}(\mathrm{x}, \mathrm{y})
\end{aligned}
$$

This also is valid for a single photon. In the directions perpendicular to the direction of propagation, the magnetic radiation pressures (49) and (51) have been compensated completely by the magnetic interaction forces (48) and (51). 


\subsection{Gravitational-Electromagnetic Confinement}

Calculations in Mathematica 11.0 .nb file and in PDF file.

The Gravitational-Electromagnetic Confinement for the elementary structure beyond the concept of the "superstring model" is presented in (8).

$$
\begin{aligned}
& -\frac{1}{c^{2}} \frac{\partial(\bar{E} \times \bar{H})}{\partial t}+\varepsilon_{0} \bar{E}(\nabla . \bar{E})-\varepsilon_{0} \bar{E} \times(\nabla \times \bar{E})+\mu_{0} \bar{H}(\nabla \cdot \bar{H})- \\
& -\mu_{0} \bar{H} \times(\nabla \times \bar{H})-\frac{1}{2} \varepsilon_{0}^{2} \mu_{0}(\bar{E} . \bar{E}) \bar{g}-\frac{1}{2} \varepsilon_{0} \mu_{0}^{2}(\bar{H} \cdot \bar{H}) \bar{g}=\overline{0}
\end{aligned}
$$

In which $\bar{g}$ represents the (radial oriënted) gravitational $\overline{\mathrm{g}}(\theta, r, \varphi)$ acceleration caused by the electromagnetic mass density $\bar{\rho}(\theta, r, \varphi)$ of the confined electromagnetic radiation. One of the many possible solutions for Gravitational Electromagnetic Equation (52) equals:

$$
\begin{aligned}
& \left(\begin{array}{l}
e_{r} \\
e_{\theta} \\
e_{\varphi}
\end{array}\right)=\left(\begin{array}{c}
0 \\
\mathrm{f}(\mathrm{r}) \operatorname{Sin}(\omega \mathrm{t}) \\
-\mathrm{f}(\mathrm{r}) \operatorname{Cos}(\omega \mathrm{t})
\end{array}\right),\left(\begin{array}{l}
m_{r} \\
m_{\theta} \\
m_{\varphi}
\end{array}\right)=\left(\begin{array}{c}
0 \\
\mathrm{f}(\mathrm{r}) \operatorname{Cos}(\omega \mathrm{t}) \\
\mathrm{f}(\mathrm{r}) \operatorname{Sin}(\omega \mathrm{t})
\end{array}\right) \\
& \mathrm{w}_{\mathrm{em}}=\left(\frac{\mu_{0}}{2}(\overline{\mathrm{m}} \cdot \overline{\mathrm{m}})+\frac{\varepsilon_{0}}{2}(\overline{\mathrm{e}} \cdot \overline{\mathrm{e}})\right)=\varepsilon_{0} \mathrm{f}(r)^{2}
\end{aligned}
$$

In which $\boldsymbol{\omega}$ represents an arbitrary frequency of a sinusoidal function and the radial function $\mathrm{f}(\mathrm{r})$ equals:

$$
f[r]=K \mathrm{e}^{-\frac{G 1 \varepsilon_{0} \mu_{0}}{r}+8 \pi \log [r]} \frac{-8 \pi}{8 \pi}
$$




\subsection{Gravitational-Electromagnetic Confinement: Type $1(\mathrm{emm}=$} $\left.10^{-4}[\mathrm{~kg}], \mathbf{r}=21^{-35}[\mathrm{~m}]\right)$

To realize a Gravitational-Electromagnetic confinement of an Electro Magnetic Mass emm $=10^{-4}[\mathrm{~kg}]$, the solution for the Gravitational Electromagnetic Equation (53) results in a Gravitational Electromagnetic Confinement radius $\mathrm{r}=210^{-35}[\mathrm{~m}]$.

$$
\begin{aligned}
& f[r]=K \mathrm{e}^{-\frac{-\frac{G 1 \mathrm{emm} \varepsilon_{0} \mu_{0}}{r}+8 \pi \log [r]}{8 \pi}} \\
& G 1=6.6740810^{-11} \\
& \mathrm{emm}=10^{-4} \\
& \varepsilon_{0}=8.8510^{-12} \\
& \mu_{0}=1.256637061435917210^{-6}
\end{aligned}
$$

In which "emm" equals the electromagnetic mass of the Gravitational Electromagnetic Confinement located at the center according Newton's Shell Theorem.

For an electromagnetic mass of the confinement type (1): emm $=10^{-4}$ $[\mathrm{kg}]$, the radius of the confinement equals approximately $2 \times 10^{-35}[\mathrm{~m}]$. This is in the order of Planck's Length,

The Plot graph of the Electric Field Intensity $f(r)$ of the confinement has been presented as a function of the radius in figure (7) and figure (8): 


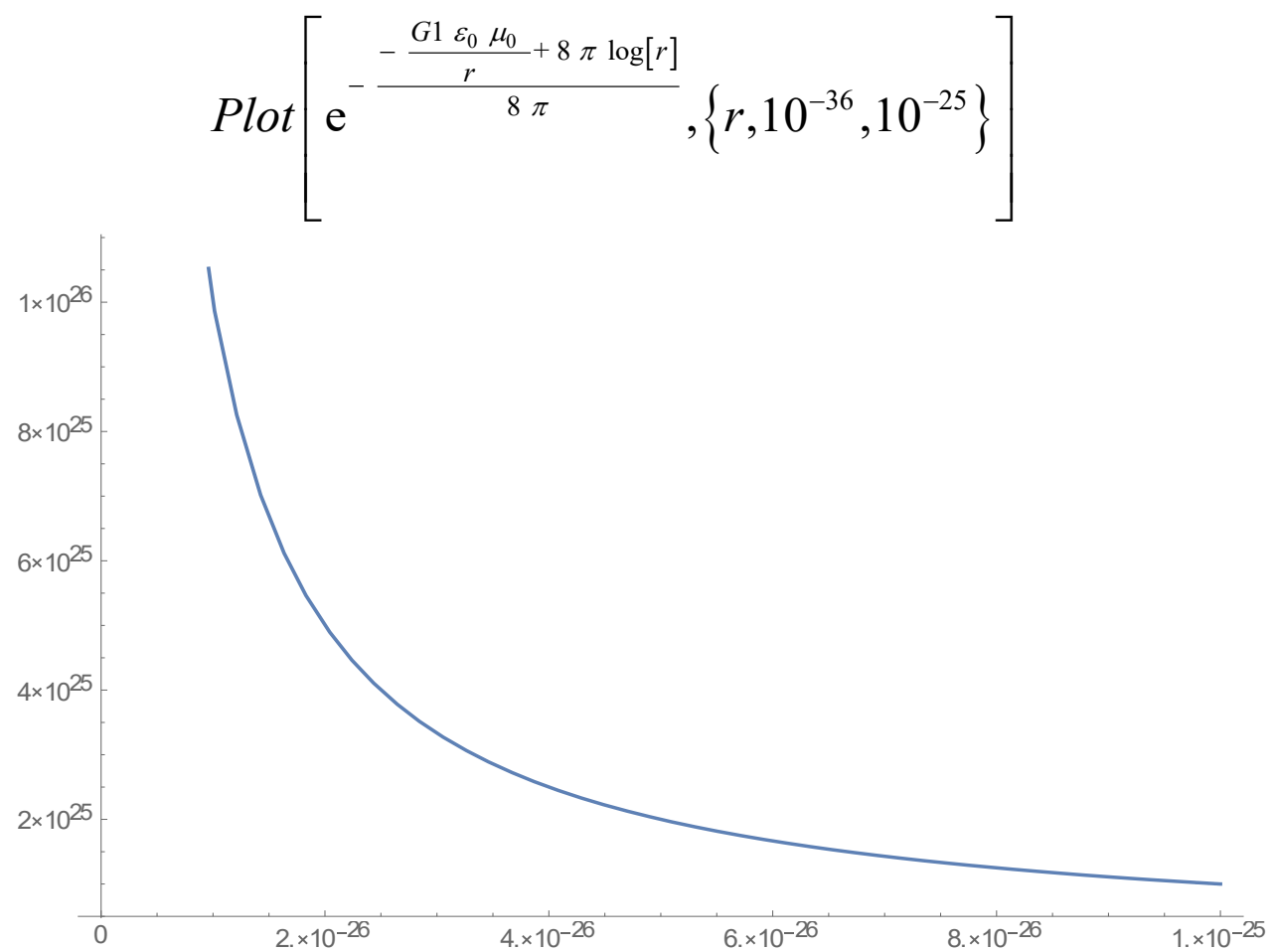

Fig. 8. PlotGraph of the Electric Field Intensity $f(r)$ for the region $10^{-36}<r<10^{-25}$ in which the gravitational field acceleration has been chosen accordingly an electromagnetic mass of $10^{-4}[\mathrm{~kg}]$ located at the center of the confinement, according Newton's Shell Theorem. 
$\operatorname{Plot}\left[\mathrm{e}^{-\frac{-\frac{G 1 \varepsilon_{0} \mu_{0}}{r}+8 \pi \log [r]}{8 \pi}},\left\{r, 10^{-36}, 10^{-35}\right\}\right]$

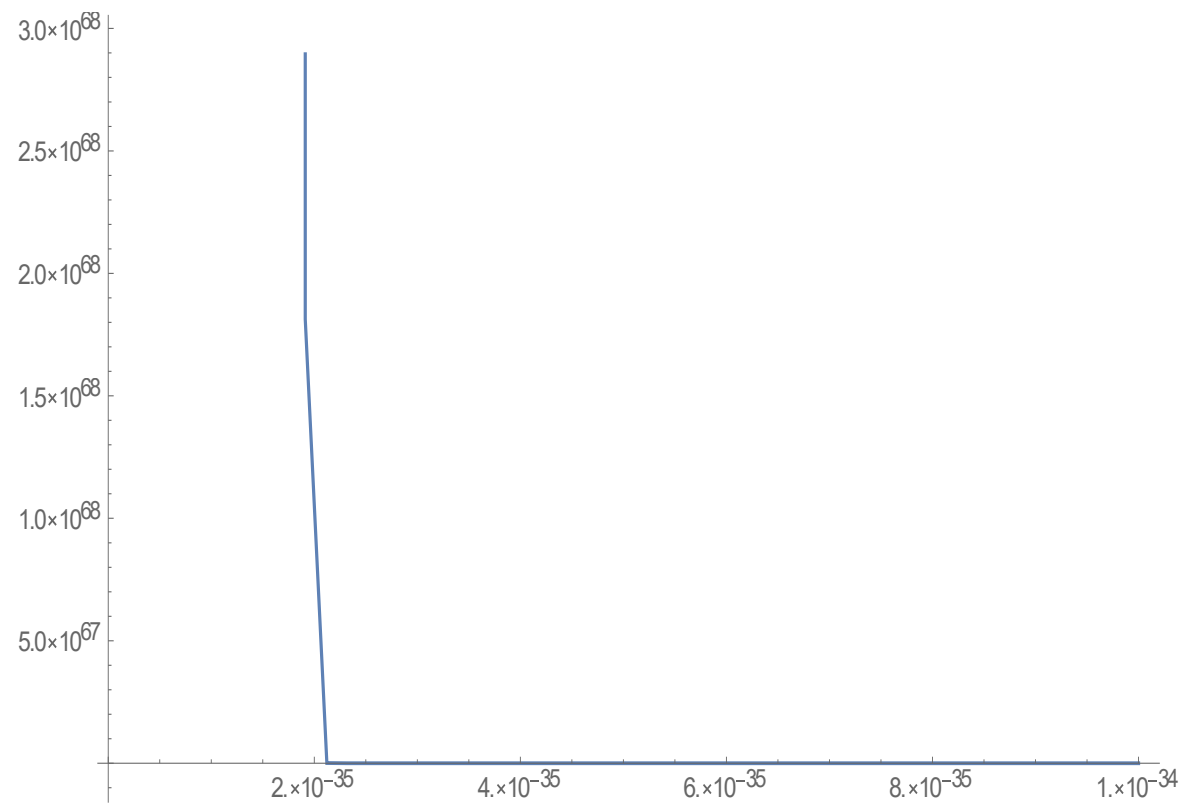

Fig. 9. PlotGraph of the Electric Field Intensity $f(r)$ for the region $10^{-36}<\mathbf{r}<1^{-35}$ in which the gravitational field acceleration has been chosen accordingly an electromagnetic mass of $10^{-4}[\mathrm{~kg}]$ located at the center of the confinement, according Newton's Shell Theorem.

It follows from Figure 9 that the radius of the stable gravitational electromagnetic confinement equals approximately $2 \times 10-35$ [m], which is the size of the Planck length. According the theory of superstrings, the fundamental constituents of reality are strings of the Planck length (about $1.6210^{-35}[\mathrm{~m}]$ ) that vibrate at resonant frequencies. 
2.18 Gravitational-Electromagnetic Confinement: Type $2(\mathrm{emm}=$ $\left.10^{-12}[\mathrm{~kg}], \mathrm{r}=2.5 \mathbf{1 0}^{-43}[\mathrm{~m}]\right)$

For an electromagnetic mass of the confinement type (2): emm $=10^{-12}$ $[\mathrm{kg}]$, the radius of the confinement equals approximately $2.5 \times 10^{-43}[\mathrm{~m}]$. This is much smaller than Planck's Length and has been indicated as "sub Planck Length".

The Plot graph of the Electric Field Intensity $f(r)$ of the confinement has been presented as a function of the radius in figure (9) and figure (10):

$\operatorname{Plot}\left[\mathrm{e}^{-\frac{-\frac{G 1 \varepsilon_{0} \mu_{0}}{r}+8 \pi \log [r]}{8 \pi}},\left\{r, 10^{-43}, 10^{-40}\right\}\right]$

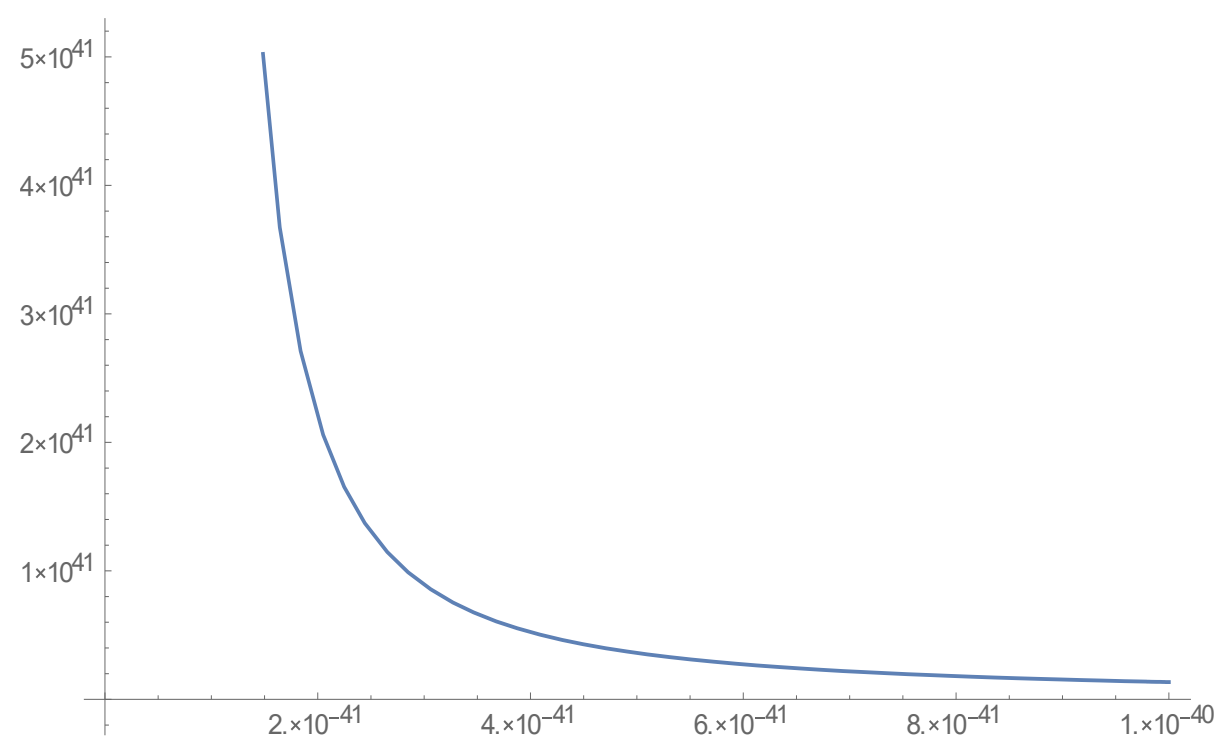

Fig. 10. PlotGraph of the Electric Field Intensity $f(r)$ for the region $10^{-43}<r<10^{-40}$ in which the gravitational field acceleration has been chosen accordingly an electromagnetic mass of $10^{-12}[\mathrm{~kg}]$ located at the center of the confinement, according Newton's Shell Theorem. 


$$
\text { Plot }\left[\mathrm{e}^{-\frac{-\frac{G 1 \varepsilon_{0} \mu_{0}}{r}+8 \pi \log [r]}{8 \pi}},\left\{r, 10^{-43}, 10^{-42}\right\}\right]
$$

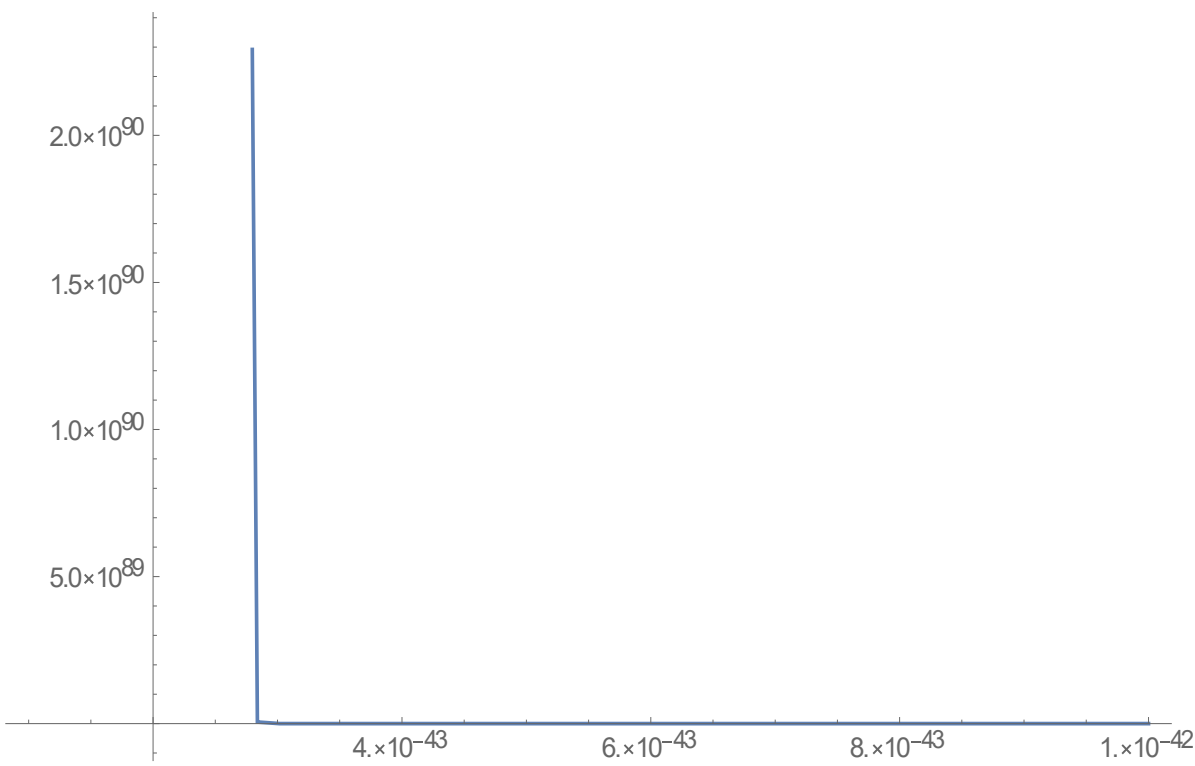

Fig. 11. PlotGraph of the Electric Field Intensity $f(r)$ for the region $10^{-43}<r<10^{-42}$ in which the gravitational field acceleration has been chosen accordingly an electromagnetic mass of $10^{-12}[\mathrm{~kg}]$ located at the center of the confinement, according Newton's Shell Theorem. 


\subsection{Gravitational-Electromagnetic Confinement: Type $3(\mathrm{emm}=$}

$\left.10^{-27}[\mathrm{~kg}], \mathbf{r}=3 \mathbf{1 0}^{-58}[\mathrm{~m}]\right)$

For an electromagnetic mass of the confinement type (3): emm = $1.6726 \times 10^{-27}[\mathrm{~kg}]$ (mass of proton), the radius of the confinement equals approximately $3 \times 10^{-58}[\mathrm{~m}]$. This is far beyond the order of Planck's Length,

The Plot graph of the Electric Field Intensity $\mathrm{f}(\mathrm{r})$ of the confinement has been presented as a function of the radius in figure (11) and figure (12):

$\operatorname{Plot}\left[\mathrm{e}^{-\frac{-\frac{G 1 \varepsilon_{0} \mu_{0}}{r}+8 \pi \log [r]}{8 \pi}},\left\{r, 10^{-59}, 10^{-55}\right\}\right]$

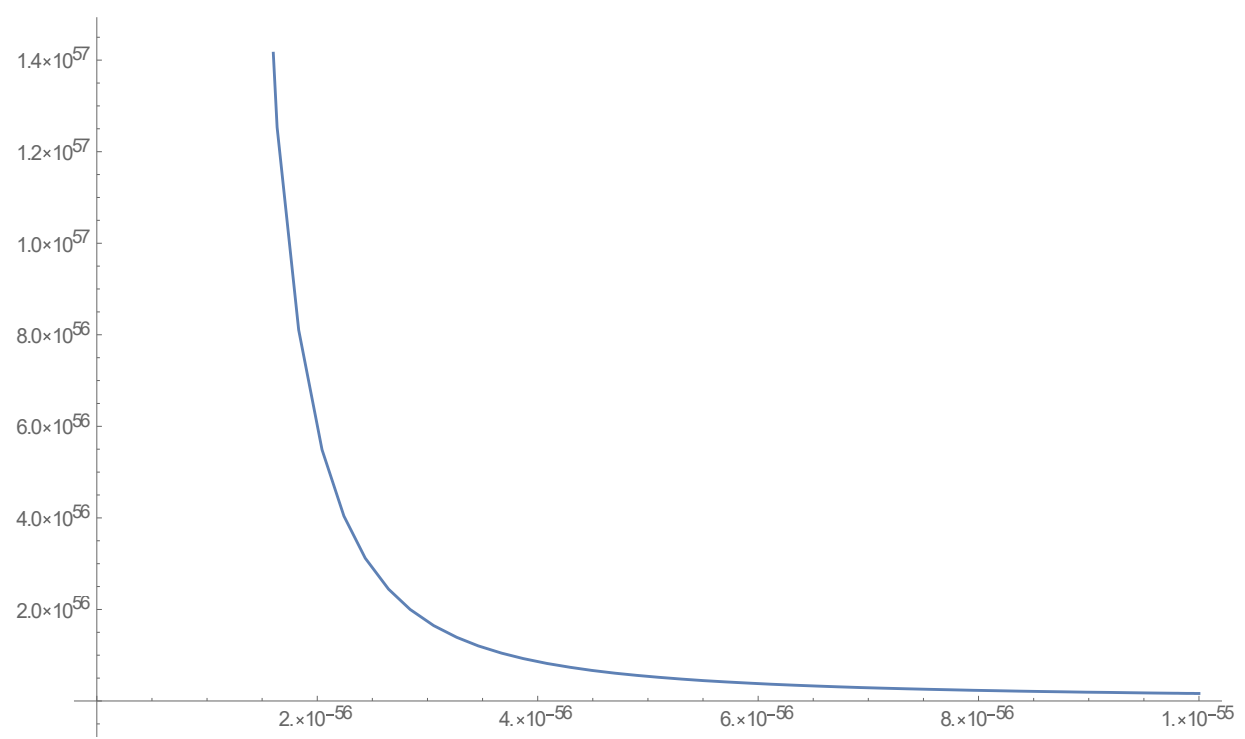

Fig. 12. PlotGraph of the Electric Field Intensity $f(r)$ for the region $10^{-59}<\mathrm{r}<10^{-55}$ in which the gravitational field acceleration has been chosen accordingly an electromagnetic mass of $1.6726 \times 10^{-27}$ $[\mathrm{kg}]$ located at the center of the confinement, according Newton's Shell Theorem. 


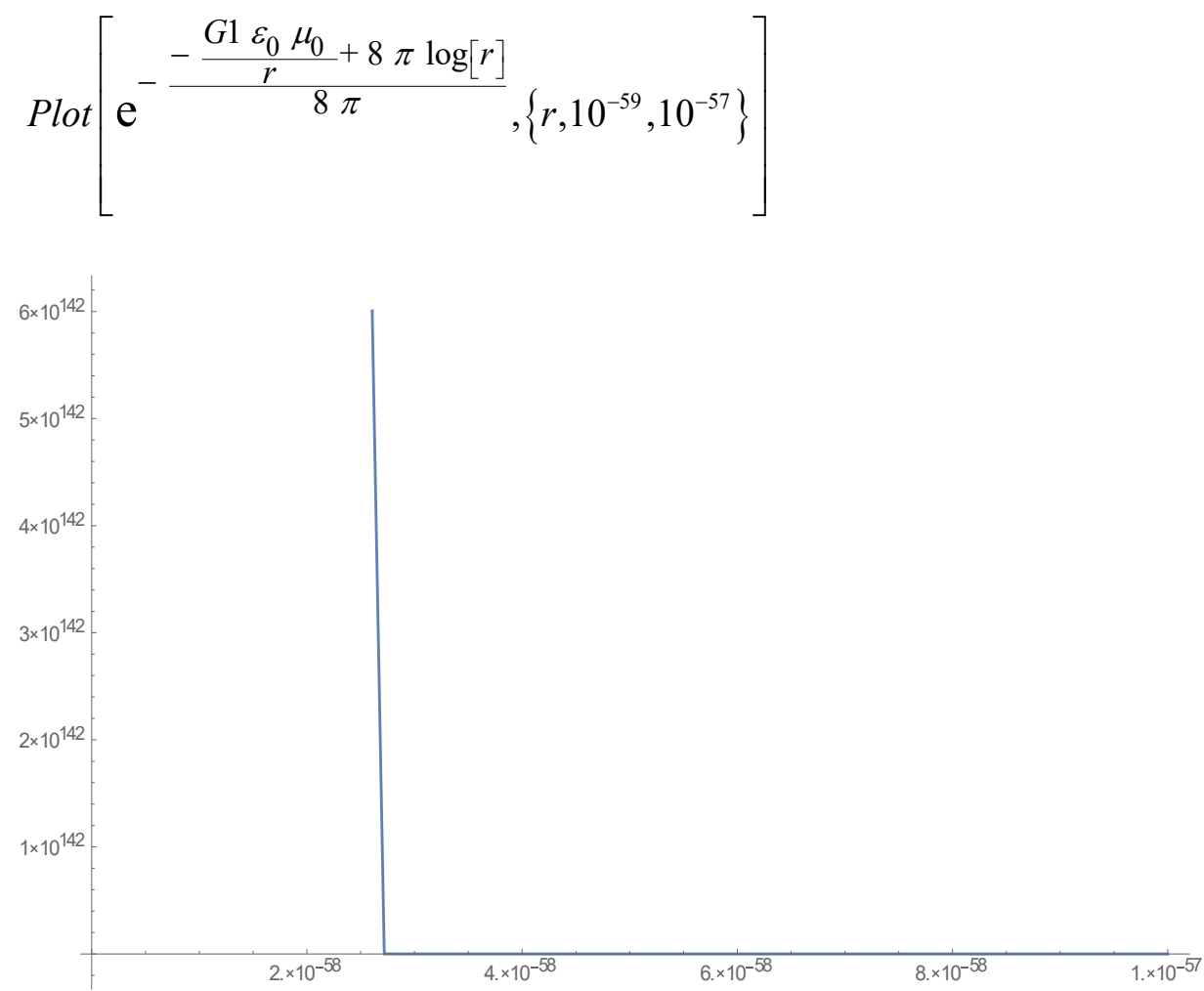

Fig. 13. PlotGraph of the Electric Field Intensity $f(r)$ for the region $10^{-59}<\mathbf{r}<10^{-57}$ in which the gravitational field acceleration has been chosen accordingly an electromagnetic mass of $1.6726 \times 10^{-27}$ [kg] located at the center of the confinement, according Newton's Shell Theorem.

The fundamental question is: How it is possible to create confinements from "visible light" (with a wave length between $3.9 \times 10^{-7}[\mathrm{~m}]$ until $7 \mathrm{x}$ $\left.10^{-7}[\mathrm{~m}]\right)$ within dimensions smaller than Planck's Length?

This is only possible when the wave-length of the confined radiation is smaller than de dimensions of the confinement. This requires extreme high frequencies. The transformation in frequency from visible light into the extreme high frequency of the confinement is possible because of the combined Lorentz transformation/ Doppler Effect during the collapse (contraction) of the radiation when the confinement has been formed (Implosion of Visible Light). 


\subsection{The Origin of Electric Charge and Magnetic Spin in Discrete Values}

The Gravitational-Electromagnetic Confinement for the elementary structure of the "superstring" is presented in equation (5-a).

$$
\begin{aligned}
& -\frac{1}{c^{2}} \frac{\partial(\bar{E} \times \bar{H})}{\partial t}+\varepsilon_{0} \bar{E}(\nabla \cdot \bar{E})-\varepsilon_{0} \bar{E}_{\times(\nabla \times \bar{E})+\mu_{0}} \bar{H}(\nabla \cdot \bar{H})- \\
& -\mu_{0} \bar{H} \times(\nabla \times \bar{H})-\frac{1}{2} \varepsilon_{0}^{2} \mu_{0}(\bar{E} \cdot \bar{E}) \overline{\mathrm{g}}-\frac{1}{2} \varepsilon_{0} \mu_{0}^{2}(\bar{H} \cdot \bar{H}) \overline{\mathrm{g}}=\overline{0}
\end{aligned}
$$

In which $\bar{g}$ represents the (radial oriented) gravitational $\bar{g}(\theta, r, \varphi)$ acceleration caused by the electromagnetic mass density $\bar{\rho}(\theta, r, \varphi)$ of the confined electromagnetic radiation. One of the many possible solutions for Gravitational Electromagnetic Equation (52) equals:

To find the origin of Electric Charge and Magnetic Spin we choose as an example the solution for equation (52) which equals:

$$
\begin{aligned}
& \left(\begin{array}{l}
e_{r} \\
e_{\theta} \\
e_{\varphi}
\end{array}\right)=\left(\begin{array}{c}
0 \\
\mathrm{f} 1(\mathrm{r}, \theta, \varphi, \mathrm{t}) \operatorname{Sin}(\omega \mathrm{t}) \\
-\mathrm{f} 2(\mathrm{r}, \theta, \varphi, \mathrm{t}) \operatorname{Cos}(\omega \mathrm{t})
\end{array}\right),\left(\begin{array}{l}
m_{r} \\
m_{\theta} \\
m_{\varphi}
\end{array}\right)=\left(\begin{array}{c}
0 \\
\mathrm{f} 2(\mathrm{r}, \theta, \varphi, \mathrm{t}) \operatorname{Cos}(\omega \mathrm{t}) \\
\mathrm{f} 1(\mathrm{r}, \theta, \varphi, \mathrm{t}) \operatorname{Sin}(\omega \mathrm{t})
\end{array}\right) \\
& \mathrm{w}_{\mathrm{em}}=\left(\frac{\mu_{0}}{2}(\overline{\mathrm{m}} \cdot \overline{\mathrm{m}})+\frac{\varepsilon_{0}}{2}(\overline{\mathrm{e}} \cdot \overline{\mathrm{e}})\right)=\varepsilon_{0} \mathrm{f}(r)^{2}
\end{aligned}
$$

In which $\mathrm{f}[\mathrm{r}], f 1[r, \theta, \varphi, t], f 2[r, \theta, \varphi, t]$ equals:

$$
\begin{aligned}
& f[r]=K \mathrm{e}^{-\frac{-\frac{G 1 \varepsilon_{0} \mu_{0}}{r}+8 \pi \log [r]}{8 \pi}} \\
& f 1[r, \theta, \varphi, t]=K \mathrm{e}^{-\frac{-\frac{G 1 \varepsilon_{0} \mu_{0}}{r}+8 \pi \log [r]}{8 \pi}} g 1[\theta, \varphi, t] \\
& f 2[r, \theta, \varphi, t]=\frac{K \mathrm{e}^{-\frac{-\frac{G 1 \varepsilon_{0} \mu_{0}}{r}+8 \pi \log [r]}{8 \pi}} \sqrt{-g 1[\theta, \varphi, t]^{2}+\cos [2 t \omega] g 1[\theta, \varphi, t]^{2}+2 h[\theta, \varphi]}}{\sqrt{2}}
\end{aligned}
$$


In which $g 1[\theta, \varphi, t]$ and $\mathrm{h}[\theta, \varphi]$ are arbitrary function.

The "sub Max Planck's length" confinement has been described for the electric field intensity:

$$
\left(\begin{array}{c}
e_{r} \\
e_{\theta} \\
e_{\varphi}
\end{array}\right)=\left(\begin{array}{c}
0 \\
K \mathrm{e}^{-\frac{-\frac{G 1 \varepsilon_{0} \mu_{0}}{r}+8 \pi \log [r]}{8 \pi}} g 1[\theta, \varphi, t] \sin [t \omega] \\
-\frac{K \mathrm{e}^{-\frac{-\frac{G 1 \varepsilon_{0} \mu_{0}}{r}+8 \pi \log [r]}{8 \pi}} \sqrt{-g 1[\theta, \varphi, t]^{2}+\cos [2 t \omega] g 1[\theta, \varphi, t]^{2}+2 h[\theta, \varphi]}}{\sqrt{2}}
\end{array}\right)
$$

The "sub Max Planck's length" confinement has been described for the magnetic field intensity:

$$
\left(\begin{array}{l}
m_{r} \\
m_{\theta} \\
m_{\varphi}
\end{array}\right)=\sqrt{\frac{\varepsilon_{0}}{\mu_{0}}}\left(\begin{array}{c}
\frac{K \mathrm{e}^{-\frac{-\frac{G 1 \varepsilon_{0} \mu_{0}}{r}+8 \pi \log [r]}{8 \pi}} \sqrt{-g 1[\theta, \varphi, t]^{2}+\cos [2 t \omega] g 1[\theta, \varphi, t]^{2}+2 h[\theta, \varphi]}}{\sqrt{2}} \\
K \mathrm{e}^{-\frac{-\frac{G 1 \varepsilon_{0} \mu_{0}}{r}+8 \pi \log [r]}{8 \pi}} g 1[\theta, \varphi, t] \sin [t \omega]
\end{array}\right)
$$




\subsection{Types of "Sub Max Planck Length Gravitational-} Electromagnetic Confinements" resulting in Electric Charge and Magnetic Spin

The following functions with the quantum variables $\{\mathrm{m} 1, \mathrm{n} 1, \mathrm{p} 1, \mathrm{q} 1\}$ have been chosen:

$$
\begin{aligned}
& f[r]=K \mathrm{e}^{-\frac{-\frac{G 1 \varepsilon_{0} \mu_{0}}{r}+8 \pi \log [r]}{8 \pi}} \\
& \mathrm{g} 1(\theta, \varphi, t)=\sin (t \omega)(\sin (\pi \theta \mathrm{m} 1) \sin (\mathrm{n} 12 \pi \varphi)+1) \\
& h(\theta, \varphi)=\sin (\pi \theta \mathrm{p} 1) \sin (\mathrm{q} 12 \pi \varphi)+1 \\
& \mathrm{~g} 2(\theta, \varphi, t)=\frac{\sec (t \omega) \sqrt{\cos (2 t \omega) \mathrm{g} 1(\theta, \varphi, t)^{2}-\mathrm{g} 1(\theta, \varphi, t)^{2}+2 h(\theta, \varphi)}}{\sqrt{2}} \\
& f 1[r, \theta, \varphi, t]=\mathrm{e}^{-\frac{-\frac{G 1 \varepsilon_{0} \mu_{0}}{r}+8 \pi \log [r]}{8 \pi}} K \operatorname{gl}[\theta, \varphi, t] \\
& f 2[r, \theta, \varphi, t]=\frac{\mathrm{e}^{-\frac{-\frac{G 1 \varepsilon_{0} \mu_{0}}{r}+8 \pi \log [r]}{8 \pi}} K \sqrt{-g 1[\theta, \varphi, t]^{2}+\cos [2 t \omega] g 1[\theta, \varphi, t]^{2}+2 h[\theta, \varphi]}}{\sqrt{2}}
\end{aligned}
$$




\subsubsection{Type 1 of "Sub Max Planck Length Gravitational-}

Electromagnetic Confinement" (Electric- and Magnetic Dipoles, Electric- and Magnetic Spin) $\{\mathrm{m1}=0, \mathrm{n} 1=0, \mathrm{p} 1=0$, $\mathbf{q} \mathbf{1}=\mathbf{0}\}$

The divergence (Electric Charge Density) of the electric field intensity (electric charge density) equals:

$\nabla .\left(\begin{array}{l}e_{r} \\ e_{\theta} \\ e_{\varphi}\end{array}\right)=\frac{\sqrt{2} \mathrm{~K} 1 \cot (\theta) \sin ^{2}(t \omega) \sqrt{1-\sin ^{4}(t \omega)} \mathrm{e}^{\frac{G_{1} \varepsilon_{0} \mu_{0}}{8 \pi \mathrm{r}}}}{r^{2} \sqrt{2-2 \sin ^{4}(t \omega)}}$

$\nabla .\left(\begin{array}{l}e_{r} \\ e_{\theta} \\ e_{\varphi}\end{array}\right)=\frac{\frac{1}{2} \mathrm{~K} 1 \cot (\theta) \mathrm{e}^{\frac{G_{1} \varepsilon_{0} \mu_{0}}{8 \pi \mathrm{r}}}}{r^{2}}$ (averaged over 1 period of time)

The divergence (Magnetic Charge Density) of the magnetic field intensity (magnetic monopole) equals:

$\nabla .\left(\begin{array}{l}m_{r} \\ m_{\theta} \\ m_{\varphi}\end{array}\right)=\frac{\mathrm{K} 1 \sqrt{\varepsilon_{0}} \cot (\theta) \sqrt{2-2 \sin ^{4}(t \omega)} \mathrm{e}^{\frac{G_{1} \varepsilon_{0} \mu_{0}}{8 \pi \mathrm{r}}}}{\sqrt{2} \sqrt{\mu 0} r^{2}}$

$\nabla .\left(\begin{array}{l}m_{r} \\ m_{\theta} \\ m_{\varphi}\end{array}\right)=\frac{\mathrm{K} 1 \sqrt{\varepsilon_{0}} \cot (\theta) \sqrt{\frac{3}{4}} \mathrm{e}^{\frac{G_{1} \varepsilon_{0} \mu_{0}}{8 \pi \mathrm{r}}}}{\sqrt{\mu 0} r^{2}}$ (averaged over 1 period of time)

In which $\mathrm{K} 1$ is an arbitrary variable. Because of the $\operatorname{Cot}(\theta)$ function, the electric divergence as well as the magnetic divergence changes from sign when the angle $\theta$ varies between $0^{\circ}$ until $360^{\circ}$ forming electric dipoles (+ versus -) and magnetic dipoles ( $\mathrm{N}$ versus $\mathrm{S}$ ). 


\subsubsection{Type 2 of "Sub Max Planck Length Gravitational-}

Electromagnetic Confinements" (Electric- and Magnetic Dipoles, Electric- and Magnetic Spin) $\{\mathrm{m1} 1=1, \mathrm{n} 1=0, \mathrm{p} 1=0$, $\mathbf{q} \mathbf{1}=\mathbf{0}\}$

The divergence of the electric field intensity (electric charge density) equals:

$\nabla \cdot\left(\begin{array}{l}e_{r} \\ e_{\theta} \\ e_{\varphi}\end{array}\right)=\frac{\sqrt{2} \mathrm{~K} 1 \cot (\theta) \sin ^{2}(t \omega) \sqrt{1-\sin ^{4}(t \omega)} \mathrm{e}^{\frac{G_{1} \varepsilon_{0} \mu_{0}}{8 \pi \mathrm{r}}}}{r^{2} \sqrt{2-2 \sin ^{4}(t \omega)}}$

$\nabla .\left(\begin{array}{l}e_{r} \\ e_{\theta} \\ e_{\varphi}\end{array}\right)=\frac{\frac{1}{2} \mathrm{~K} 1 \cot (\theta) \mathrm{e}^{\frac{G_{1} \varepsilon_{0} \mu_{0}}{8 \pi \mathrm{r}}}}{r^{2}}$ (averaged over 1 period of time)

The divergence of the magnetic field intensity (magnetic monopole) equals:

$\nabla .\left(\begin{array}{l}m_{r} \\ m_{\theta} \\ m_{\varphi}\end{array}\right)=\frac{\mathrm{K} 1 \sqrt{\varepsilon_{0}} \cot (\theta) \sqrt{2-2 \sin ^{4}(t \omega)} \mathrm{e}^{\frac{G_{1} \varepsilon_{0} \mu_{0}}{8 \pi \mathrm{r}}}}{\sqrt{2} \sqrt{\mu_{0}} r^{2}}$

$\nabla .\left(\begin{array}{l}m_{r} \\ m_{\theta} \\ m_{\varphi}\end{array}\right)=\frac{\mathrm{K} 1 \sqrt{\frac{3}{4} \sqrt{\varepsilon_{0}}} \cot (\theta) \mathrm{e}^{\frac{G_{1} \varepsilon_{0} \mu_{0}}{8 \pi \mathrm{r}}}}{\sqrt{\mu_{0}} r^{2}}$

(averaged over 1 period of time)

In which $\mathrm{K} 1$ is an arbitrary variable. Because of the $\operatorname{Cot}(\theta)$ function, the electric divergence as well as the magnetic divergence changes from sign when the angle $\theta$ varies between $0^{\circ}$ until $360^{\circ}$ forming electric dipoles (+ versus -) and magnetic dipoles ( $\mathrm{N}$ versus $\mathrm{S}$ ). 


\subsubsection{Type 3 of "Sub Max Planck Length Gravitational-} Electromagnetic Confinements" $\{\mathrm{m} 1=1, \mathrm{n} 1=1, \mathrm{p} 1=0, \mathrm{q} 1=0\}$

The divergence of the electric field intensity (electric charge density) equals:

$\nabla .\left(\begin{array}{l}e_{r} \\ e_{\theta} \\ e_{\varphi}\end{array}\right)=\frac{\mathrm{K} 1 \sin ^{2}(t \omega) \mathrm{e}^{\frac{G_{1} \varepsilon_{0} \mu_{0}}{8 \pi \mathrm{r}}\left(\cos (\varphi)(\sin (\theta) \sin (\varphi)+1) \sin ^{2}(t \omega)\right)}}{r^{2} \sqrt{1-(\sin (\theta) \sin (\varphi)+1)^{2} \sin ^{4}(t \omega)}}+$

$\frac{\mathrm{K} 1 \sin ^{2}(t \omega) \mathrm{e}^{\frac{G_{1} \varepsilon_{0} \mu_{0}}{8 \pi \mathrm{r}}}\left((2 \cos (\theta) \sin (\varphi)+\cot (\theta)) \sqrt{1-(\sin (\theta) \sin (\varphi)+1)^{2} \sin ^{4}(t \omega)}\right)}{r^{2} \sqrt{1-(\sin (\theta) \sin (\varphi)+1)^{2} \sin ^{4}(t \omega)}}$

$\nabla .\left(\begin{array}{l}e_{r} \\ e_{\theta} \\ e_{\varphi}\end{array}\right)=\frac{\left.\mathrm{K} 1 \mathrm{e}^{\frac{G_{1} \varepsilon_{0} \mu_{0}}{8 \pi \mathrm{r}}\left(\frac{1}{2} \cos (\varphi)(\sin (\theta) \sin (\varphi)+1)\right.}\right)}{2 r^{2} \sqrt{1-\frac{1}{4}(\sin (\theta) \sin (\varphi)+1)^{2}}}+$

$\mathrm{K} 1 \mathrm{e}^{\frac{G_{1} \varepsilon_{0} \mu_{0}}{8 \pi \mathrm{r}}\left((2 \cos (\theta) \sin (\varphi)+\cot (\theta)) \sqrt{1-\frac{1}{4}(\sin (\theta) \sin (\varphi)+1)^{2}}\right)}$

$$
2 r^{2} \sqrt{1-\frac{1}{4}(\sin (\theta) \sin (\varphi)+1)^{2}}
$$

(averaged over 1 period of time) 
The divergence of the magnetic field intensity (magnetic monopole) equals:

$\nabla .\left(\begin{array}{l}m_{r} \\ m_{\theta} \\ m_{\varphi}\end{array}\right)=\frac{-\mathrm{K} 1 \sqrt{\varepsilon_{0}} \mathrm{e}^{\frac{G_{1} \varepsilon_{0} \mu_{0}}{8 \pi \mathrm{r}}\left(\sin (\varphi) \sin ^{4}(t \omega)(\sin (2 \theta) \sin (\varphi))\right.}}{\sqrt{\mu_{0}} r^{2} \sqrt{1-(\sin (\theta) \sin (\varphi)+1)^{2} \sin ^{4}(t \omega)}}+$

$\frac{\left.\mathrm{K} 1 \sqrt{\varepsilon_{0}} \mathrm{e}^{\frac{G_{1} \varepsilon_{0} \mu_{0}}{8 \pi \mathrm{r}}}(3 \cos (\theta))+\cos (\varphi) \sin ^{2}(t \omega) \sqrt{1-(\sin (\theta) \sin (\varphi)+1)^{2} \sin ^{4}(t \omega)}-\cot (\theta)\left(\sin ^{4}(t \omega)-1\right)\right)}{\sqrt{\mu_{0}} r^{2} \sqrt{1-(\sin (\theta) \sin (\varphi)+1)^{2} \sin ^{4}(t \omega)}}$

$\nabla .\left(\begin{array}{l}m_{r} \\ m_{\theta} \\ m_{\varphi}\end{array}\right)=\frac{-\mathrm{K} 1 \sqrt{\varepsilon_{0}} \mathrm{e}^{\frac{G_{1} \varepsilon_{0} \mu_{0}}{8 \pi \mathrm{r}}}\left(\sin (\varphi) \frac{1}{4}(\sin (2 \theta) \sin (\varphi)+3 \cos (\theta))\right)}{\sqrt{\mu_{0}} r^{2} \sqrt{1-\frac{1}{4}(\sin (\theta) \sin (\varphi)+1)^{2}}}+$

$$
\frac{\mathrm{K} 1 \sqrt{\varepsilon_{0}} \mathrm{e}^{\frac{G_{1} \varepsilon_{0} \mu_{0}}{8 \pi \mathrm{r}}}\left(\frac{1}{2} \cos (\varphi) \sqrt{1-\frac{1}{4}(\sin (\theta) \sin (\varphi)+1)^{2}}+\frac{3}{4} \cot (\theta)\right)}{\sqrt{\mu_{0}} r^{2} \sqrt{1-\frac{1}{4}(\sin (\theta) \sin (\varphi)+1)^{2}}}
$$

(averaged over 1 period of time)

In which $\mathrm{K} 1$ is an arbitrary variable with a Positive (positive charge) or a Negative (negative charge) value. 
2.21.4 Type 4 of "Sub Max Planck Length GravitationalElectromagnetic Confinements" (Electric- and Magnetic Dipoles, Electric- and Magnetic Spin) $\{\mathrm{m1} 1=0, \mathrm{n} 1=0, \mathrm{p} 1=1$, q1 $=0$ \}

The divergence of the electric field intensity (electric charge density) equals:

$\nabla .\left(\begin{array}{l}e_{r} \\ e_{\theta} \\ e_{\varphi}\end{array}\right)=\frac{\mathrm{e}^{\frac{G_{1} \varepsilon_{0} \mu_{0}}{8 \pi \mathrm{r}}} K 1 \cot [\theta] \sin [t \omega]^{2}}{r^{2}}$

$\nabla .\left(\begin{array}{l}e_{r} \\ e_{\theta} \\ e_{\varphi}\end{array}\right)=\frac{\mathrm{e}^{\frac{G_{1} \varepsilon_{0} \mu_{0}}{8 \pi \mathrm{r}}} K 1 \cot [\theta]}{2 r^{2}}$

(averaged over 1 period of time)

In which $\mathrm{K} 1$ is an arbitrary variable with a Positive (positive charge) or a Negative (negative charge) value. 


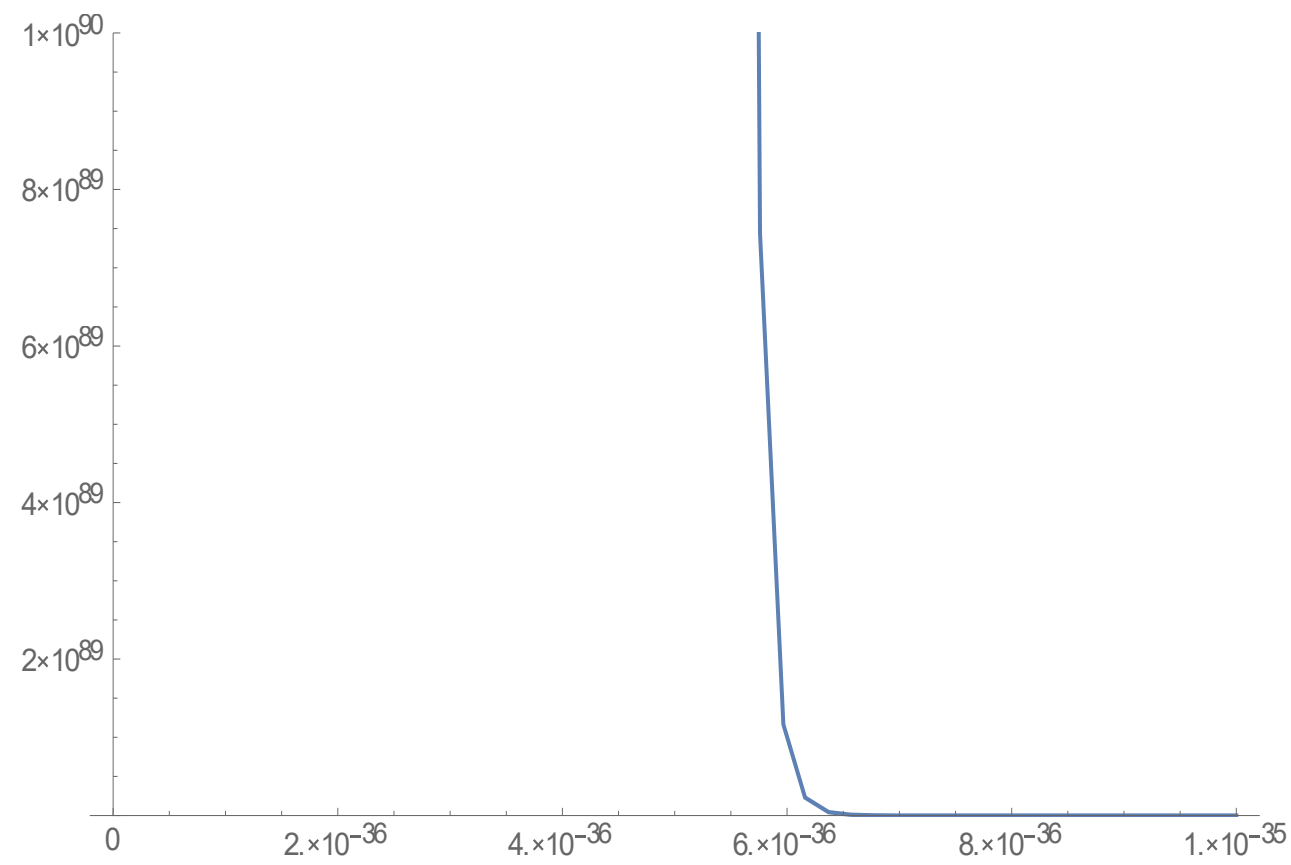

Fig. 12. Equation (66). Averaged over 1 period of time. Electric Charge Density Plot in a range from $10^{-37}[\mathrm{~m}]$ until $10^{-35}[\mathrm{~m}]$. 
The divergence of the magnetic field intensity (magnetic monopole) equals:

$\nabla .\left(\begin{array}{l}m_{r} \\ m_{\theta} \\ m_{\varphi}\end{array}\right)=\frac{\mathrm{e}^{\frac{G_{1} \varepsilon_{0} \mu_{0}}{8 \pi \mathrm{r}}} K 1 \sqrt{\varepsilon_{0}} \cot [\theta] \sqrt{1-\sin [t \omega]^{4}}}{r^{2} \sqrt{\mu_{0}}}$

$\nabla .\left(\begin{array}{l}m_{r} \\ m_{\theta} \\ m_{\varphi}\end{array}\right)=\frac{\mathrm{e}^{\frac{G_{1} \varepsilon_{0} \mu_{0}}{8 \pi \mathrm{r}}} K 1 \sqrt{\varepsilon_{0}} \cot [\theta]}{2 r^{2} \sqrt{\mu_{0}}}$

(averaged over 1 period of time)

In which $\mathrm{K} 1$ is an arbitrary variable. Because of the $\operatorname{Cot}(\theta)$ function, the electric divergence as well as the magnetic divergence changes from sign when the angle $\theta$ varies between $0^{\circ}$ until $360^{\circ}$ forming electric dipoles (+ versus -) and magnetic dipoles ( $\mathrm{N}$ versus $\mathrm{S})$. 


\subsubsection{Type 5 of "Sub Max Planck Length Gravitational- Electromagnetic Confinements" $\{\mathrm{m} 1=0, \mathrm{n} 1=0, \mathrm{p} 1=1, \mathrm{q} 1=1\}$}

The divergence of the electric field intensity (electric charge density) equals:

$\nabla .\left(\begin{array}{l}e_{r} \\ e_{\theta} \\ e_{\varphi}\end{array}\right)=\frac{\mathrm{e}^{\frac{G_{1} \varepsilon_{0} \mu_{0}}{8 \pi \mathrm{r}}} K 1\left(2 \cot [\theta] \sin [t \omega]^{2}-\frac{\cos [\varphi]}{\sqrt{1+\sin [\theta] \sin [\varphi]-\sin [t \omega]^{4}}}\right)}{2 r^{2}}$

$\nabla .\left(\begin{array}{c}e_{r} \\ e_{\theta} \\ e_{\varphi}\end{array}\right)=\frac{\mathrm{e}^{\frac{G_{1} \varepsilon_{0} \mu_{0}}{8 \pi \mathrm{r}}} K 1\left(\cot [\theta]-\frac{\cos [\varphi]}{\sqrt{\frac{3}{4}+\sin [\theta] \sin [\varphi]}}\right)}{2 r^{2}}$

(averaged over 1 period of time)

The divergence of the magnetic field intensity (magnetic monopole) equals:

$\nabla .\left(\begin{array}{l}m_{r} \\ m_{\theta} \\ m_{\varphi}\end{array}\right)=\frac{\mathrm{e}^{\frac{G_{1} \varepsilon_{0} \mu_{0}}{8 \pi \mathrm{r}}} K 1 \sqrt{\varepsilon_{0}}\left(3 \cos [\theta] \sin [\varphi]-2 \cot [\theta]\left(-1+\sin [t \omega]^{4}\right)\right)}{2 r^{2} \sqrt{\mu_{0}} \sqrt{1+\sin [\theta] \sin [\varphi]-\sin [t \omega]^{4}}}$

$\nabla .\left(\begin{array}{l}m_{r} \\ m_{\theta} \\ m_{\varphi}\end{array}\right)=\frac{\mathrm{e}^{\frac{G_{1} \varepsilon_{0} \mu_{0}}{8 \pi \mathrm{r}}} K 1 \sqrt{\varepsilon_{0}}\left(3 \cos [\theta] \sin [\varphi]+\frac{3}{2} \cot [\theta]\right)}{2 r^{2} \sqrt{\mu_{0}} \sqrt{\frac{3}{4}+\sin [\theta] \sin [\varphi]}}$

(averaged over 1 period of time)

In which $\mathrm{K} 1$ is an arbitrary variable with a Positive (positive charge) or a Negative (negative charge) value. 


\subsection{Type 2 of "Sub Max Planck Length Gravitational- Electromagnetic Confinements"}

The "sub Max Planck's length" Type II confinement has been described for the electric field intensity:

$$
\left(\begin{array}{l}
e_{r} \\
e_{\theta} \\
e_{\varphi}
\end{array}\right)=\left(\begin{array}{c}
0 \\
\frac{\mathrm{e}^{\frac{G 1 \varepsilon_{0} \mu_{0}}{8 \pi \mathrm{r}}} h[\theta, \varphi] \sin [\omega t]^{2} \sin \left[r \sqrt{\varepsilon_{0} \mu_{0}} \omega\right]^{2}}{r} \\
-\frac{\mathrm{e}^{\frac{G 1 \varepsilon_{0} \mu_{0}}{8 \pi \mathrm{r}}} h[\theta, \varphi] \sqrt{K 1-\sin [\omega t]^{4} \sin \left[r \sqrt{\varepsilon_{0} \mu_{0}} \omega\right]^{4}}}{r}
\end{array}\right)
$$

The "sub Max Planck's length" confinement has been described for the magnetic field intensity:

$$
\left(\begin{array}{l}
m_{r} \\
m_{\theta} \\
m_{\varphi}
\end{array}\right)=\sqrt{\frac{\varepsilon_{0}}{\mu_{0}}}\left(\begin{array}{c}
0 \\
\frac{\mathrm{e}^{\frac{G 1 \varepsilon_{0} \mu_{0}}{8 \pi \mathrm{r}}} h[\theta, \varphi] \sqrt{K 1-\sin [\omega t]^{4} \sin \left[r \sqrt{\varepsilon_{0} \mu_{0}} \omega\right]^{4}}}{r} \\
\frac{\mathrm{e}^{\frac{G 1 \varepsilon_{0} \mu_{0}}{8 \pi \mathrm{r}}} h[\theta, \varphi] \sin [\omega t]^{2} \sin \left[r \sqrt{\varepsilon_{0} \mu_{0}} \omega\right]^{2}}{r}
\end{array}\right)
$$


The divergence of the electric field intensity (electric charge density) equals:

$$
\begin{aligned}
\nabla .\left(\begin{array}{l}
e_{r} \\
e_{\theta} \\
e_{\varphi}
\end{array}\right)= & \frac{\mathrm{e}^{\frac{G 1 \epsilon 0 \mu 0}{8 \pi r}} \sqrt{K 1-\sin [t \omega]^{4} \sin \left[r \sqrt{\varepsilon_{0} \mu_{0}} \omega\right]^{4}} h^{(0,1)}[\theta, \varphi]}{r} \\
& +\frac{\mathrm{e}^{\frac{G 1 \epsilon 0 \mu 0}{8 \pi r}} \sin [t \omega]^{2} \sin \left[r \sqrt{\varepsilon_{0} \mu_{0}} \omega\right]^{2} h^{(1,0)}[\theta, \varphi]}{r}
\end{aligned}
$$

In which $\mathrm{K} 1$ is a positive constant equal or larger than 1.

The divergence of the magnetic field intensity (magnetic monopole) equals:

$$
\begin{aligned}
\nabla \cdot\left(\begin{array}{l}
m_{r} \\
m_{\theta} \\
m_{\varphi}
\end{array}\right)= & \frac{\mathrm{e}^{\frac{G_{1} \varepsilon_{0} \mu_{0}}{8 \pi \mathrm{r}}} \sqrt{\varepsilon_{0}} \sin [t \omega]^{2} \sin \left[r \sqrt{\varepsilon_{0} \mu_{0}} \omega\right]^{2} h^{(0,1)}[\theta, \varphi]}{r \sqrt{\mu_{0}}} \\
& +\frac{\mathrm{e}^{\frac{G_{1} \varepsilon_{0} \mu_{0}}{8 \pi \mathrm{r}}} \sqrt{\varepsilon_{0}} \sqrt{K 1-\sin [t \omega]^{4} \sin \left[r \sqrt{\varepsilon_{0} \mu_{0}} \omega\right]^{4}} h^{(1,0)}[\theta, \varphi]}{r \sqrt{\mu_{0}}}
\end{aligned}
$$

The function has been chosen:

$$
h[\theta, \varphi]=\sin [n \theta] \cos [m \varphi]
$$

In which the integers $\mathrm{n}=0,1 / 2,1 \frac{1}{2}, 2,2^{1 / 2}, 3,3 \frac{1}{2}, \ldots$ and $\mathrm{m}=0,1 / 2,1 \frac{1}{2}$, $2,2 \frac{1}{2}, 3,3 \frac{1}{2}, \ldots$ :

$$
\rho=\varepsilon_{0} \nabla \cdot\left(\begin{array}{l}
e_{r} \\
e_{\theta} \\
e_{\varphi}
\end{array}\right)=\frac{n \varepsilon_{0} \mathrm{e}^{\frac{G l \epsilon 0 \mu 0}{8 \pi r}} \cos (n \theta) \cos (m \varphi) \sin (t \omega)^{2} \sin \left(r \sqrt{\varepsilon_{0} \mu_{0}} \omega\right)^{2}}{r}
$$

According to Gauss's law the electric charge density $\rho$ equals for $\mathrm{m}=$ 0 and $\mathrm{n}=1$ for an Electric Monopole. Equation (75) represents a di-pole function for the electric charge density. 
For the corresponding magnetic di-pole flux density $\phi$ (spin) equals for $\mathrm{n}=0$ and $\mathrm{m}=+1 / 2$ (spin up) and $\mathrm{m}=-1 / 2(\operatorname{spin}$ down$)$ :

$$
\phi=\mu_{0} \nabla .\left(\begin{array}{l}
m_{r} \\
m_{\theta} \\
m_{\varphi}
\end{array}\right)=\frac{m \sqrt{\varepsilon_{0} \mu_{0}} \mathrm{e}^{\frac{G l \epsilon 0 \mu 0}{8 \pi r}} \cos (n \theta) \cos (m \varphi) \sin \left(r \sqrt{\varepsilon_{0} \mu_{0}} \omega\right)^{2}}{r}
$$

Equation (76) represents a di-pole function for the magnetic flux density (spin).

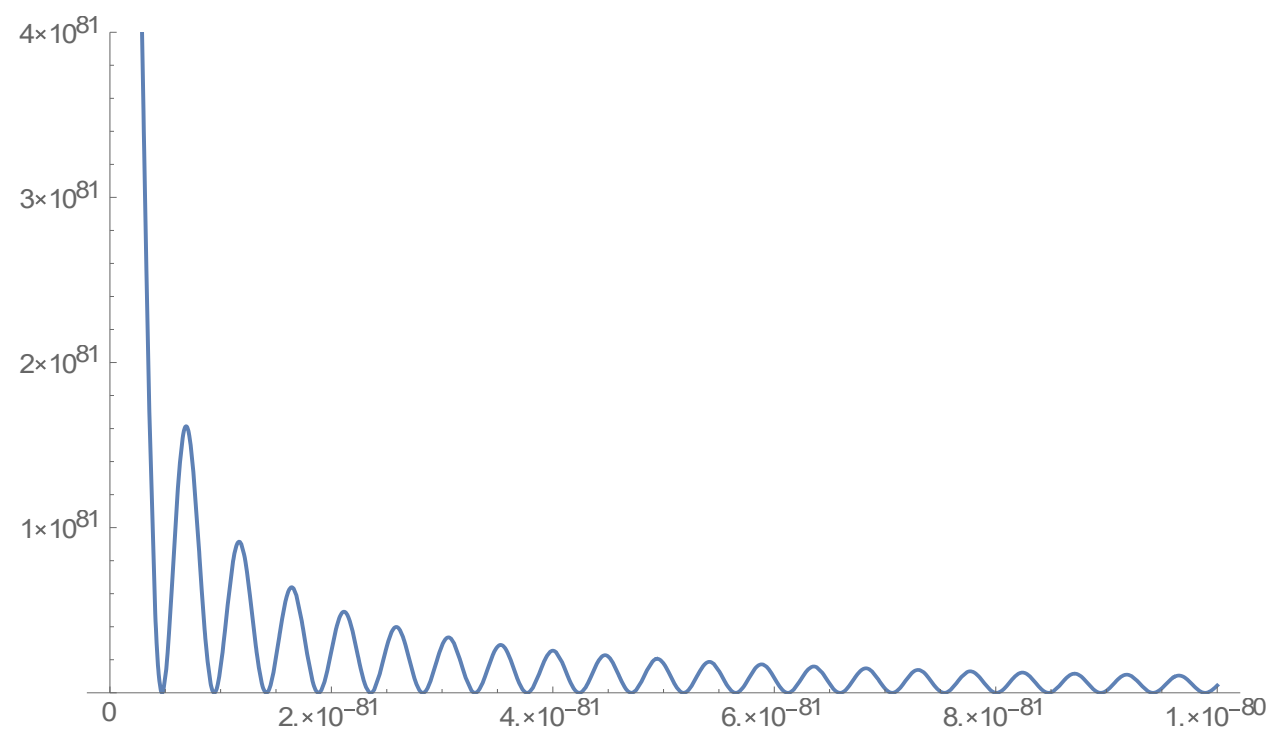

Fig. 13. Equation (56): PlotGraph of the Electric Field Intensity $f(r)$ for the region $10^{-85}<r<10^{-80}$ with a frequency of $\omega=10^{90}\left[\mathrm{~s}^{-1}\right]$ in which the gravitational field acceleration has been chosen accordingly an electromagnetic mass of $1.6726 \times 10^{-27}[\mathrm{~kg}]$ located at the center of the confinement, according Newton's Shell Theorem. 


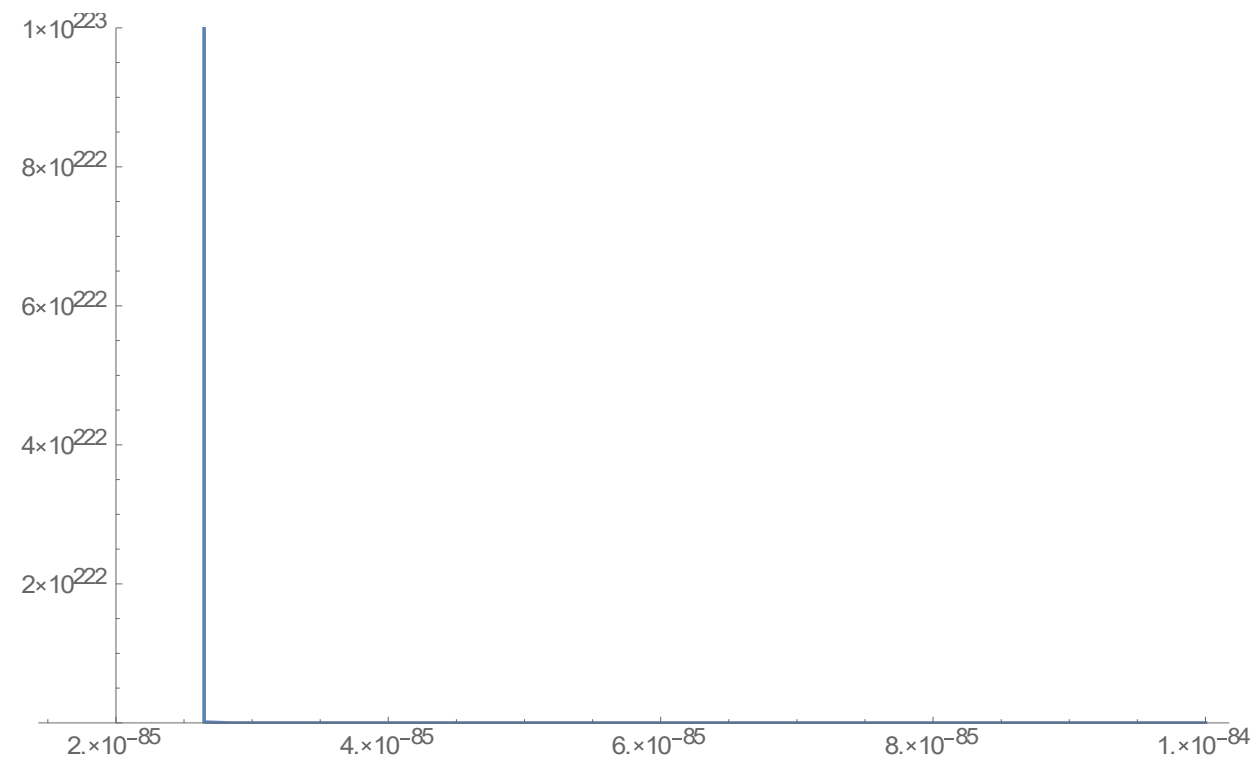

Fig.14. Equation (56): PlotGraph of the Electric Field Intensity $f(r)$ for the region $10^{-85}<r<10^{-84}$ with a frequency of $\omega=10^{90}$ in which the gravitational field acceleration has been chosen accordingly an electromagnetic mass of $1.6726 \times 10^{-27}[\mathrm{~kg}]$ located at the center of the confinement, according Newton's Shell Theorem. 


\subsection{Single Harmonic Black Holes (SHBH)}

Calculations in Mathematica 11.0 nb beyond the concept of the "Single Harmonic Black Hole" is presented in (77).

$$
\begin{aligned}
& -\frac{1}{c^{2}} \frac{\partial(\bar{E} \times \bar{H})}{\partial t}+\varepsilon_{0} \bar{E}(\nabla . \bar{E})-\varepsilon_{0} \bar{E} \times(\nabla \times \bar{E})+\mu_{0} \bar{H}(\nabla \cdot \bar{H})- \\
& -\mu_{0} \bar{H} \times(\nabla \times \bar{H})-\frac{1}{2} \varepsilon_{0}^{2} \mu_{0}(\bar{E} . \bar{E}) \bar{g}-\frac{1}{2} \varepsilon_{0} \mu_{0}^{2}(\bar{H} \cdot \bar{H}) \overline{\mathrm{g}}=\overline{0}
\end{aligned}
$$

In which $\bar{g}$ represents the (radial directed) gravitational $\bar{g}(\theta, r, \varphi)$ acceleration caused by the electromagnetic mass density $\bar{\rho}(\theta, r, \varphi)$ of the confined electromagnetic radiation. One of the possible solutions for the Single Harmonic Black Hole equals:

$$
\begin{aligned}
& \left(\begin{array}{l}
e_{r} \\
e_{\theta} \\
e_{\varphi}
\end{array}\right)=\left(\begin{array}{c}
0 \\
\mathrm{f}(\mathrm{r}) \operatorname{Sin}\left(\frac{2 \pi \mathrm{nc}}{R} \mathrm{t}\right) \\
-\mathrm{f}(\mathrm{r}) \operatorname{Cos}\left(\frac{2 \pi \mathrm{nc}}{R} \mathrm{t}\right)
\end{array}\right),\left(\begin{array}{l}
m_{r} \\
m_{\theta} \\
m_{\varphi}
\end{array}\right)=\left(\begin{array}{c}
0 \\
\mathrm{f}(\mathrm{r}) \operatorname{Cos}\left(\frac{2 \pi \mathrm{nc}}{R} \mathrm{t}\right) \\
\mathrm{f}(\mathrm{r}) \operatorname{Sin}\left(\frac{2 \pi \mathrm{nc}}{R} \mathrm{t}\right)
\end{array}\right) \\
& \mathrm{w}_{\mathrm{em}}=\left(\frac{\mu_{0}}{2}(\overline{\mathrm{m}} \cdot \overline{\mathrm{m}})+\frac{\varepsilon_{0}}{2}(\overline{\mathrm{e}} \cdot \overline{\mathrm{e}})\right)=\varepsilon_{0} \mathrm{f}(r)^{2}
\end{aligned}
$$

In which the term $\frac{2 \pi \mathrm{nc}}{R}$ represents the frequency $\omega$ of the $\mathrm{SHBH}, \mathrm{n}$ equals an integer and $\mathrm{c}$ equals the universal $\mathrm{c}=$ constant, the speed of light. Because the speed of light does not change within a longitudinal gravitational field (Equation 34), the frequency equals the speed of light divided by the radius of the Black Hole times the integer $\mathrm{n}$. The radial function $\mathrm{f}(\mathrm{r})$ equals:

$$
f[r]=K \mathrm{e}^{-\frac{G 1 \varepsilon_{0} \mu_{0}}{r}+8 \pi \log [r]}
$$


2.24 Single Harmonic Black Hole Type $1(\mathrm{emm}=1040[\mathrm{~kg}], \mathrm{r}=1.5$ $109[\mathrm{~m}], \mathbf{f}=0.2[\mathrm{~Hz}])$

To realize a Gravitational-Electromagnetic confinement of an Electro Magnetic Mass: emm $=10^{40}[\mathrm{~kg}]$, the solution for the Gravitational Electromagnetic Equilibrium Equation (79) results in a Gravitational Electromagnetic Confinement radius $\mathrm{r}=1.510^{9}[\mathrm{~m}]$ (Figure 16).

$$
\begin{aligned}
& f[r]=K \mathrm{e}^{-\frac{-\frac{G 1 \mathrm{emm} \varepsilon_{0} \mu_{0}}{r}+8 \pi \log [r]}{8 \pi}} \\
& G 1=6.6740810^{-11} \\
& \mathrm{emm}=10^{40} \\
& \varepsilon_{0}=8.8510^{-12} \\
& \mu_{0}=1.256637061435917210^{-6}
\end{aligned}
$$

In which "emm" equals the electromagnetic mass of the Single Harmonic Black Hole located at the center according Newton's Shell Theorem. For an electromagnetic mass of the SHBH $(1):$ emm $=10^{40}$ $[\mathrm{kg}]$, the radius of the confinement equals approximately $1.5 \times 10^{9}[\mathrm{~m}]$ and the first harmonic frequency equals $0.2[\mathrm{~Hz}]$. 
The Plot graph of the Electric Field Intensity $\mathrm{f}(\mathrm{r})$ of the SHBH has been presented as a function of the radius in figure (15) and figure (16):

$$
\operatorname{Plot}\left[\mathrm{e}^{-\frac{-\frac{G 1 \varepsilon_{0} \mu_{0}}{r}+8 \pi \log [r]}{8 \pi}},\left\{r, 10^{5}, 10^{12}\right\}\right]
$$

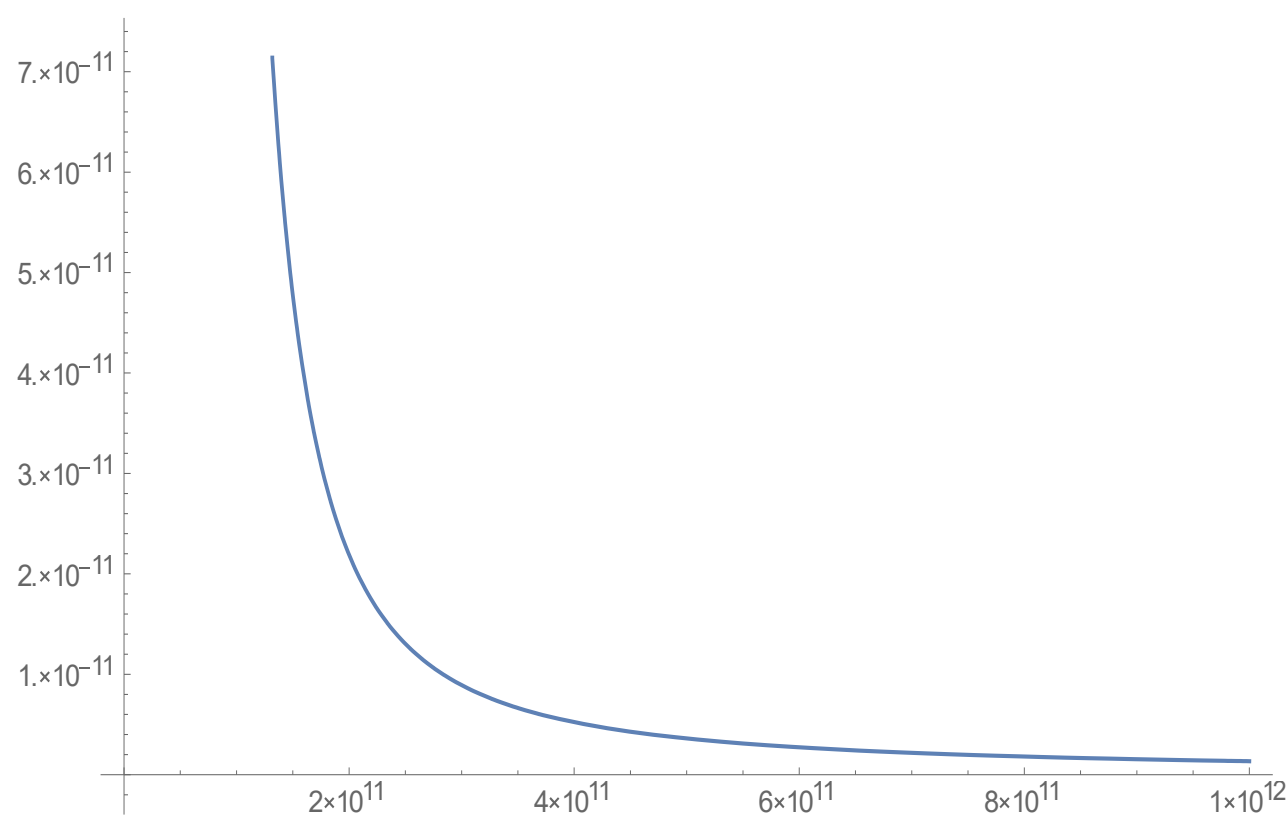

Fig. 15. PlotGraph of the Electric Field Intensity $f(r)$ (vertical axis) for the region $10^{5}<\mathrm{r}<10^{12}$ (horizontal axis) in which the gravitational field acceleration has been chosen accordingly an electromagnetic mass of $10^{40}[\mathrm{~kg}]$ located at the center of the confinement, according Newton's Shell Theorem. 
Plot $\left[\mathrm{e}^{-\frac{-\frac{G 1 \varepsilon_{0} \mu_{0}}{r}+8 \pi \log [r]}{8 \pi}},\left\{r, 10^{9}, 4 \times 10^{9}\right\}\right]$

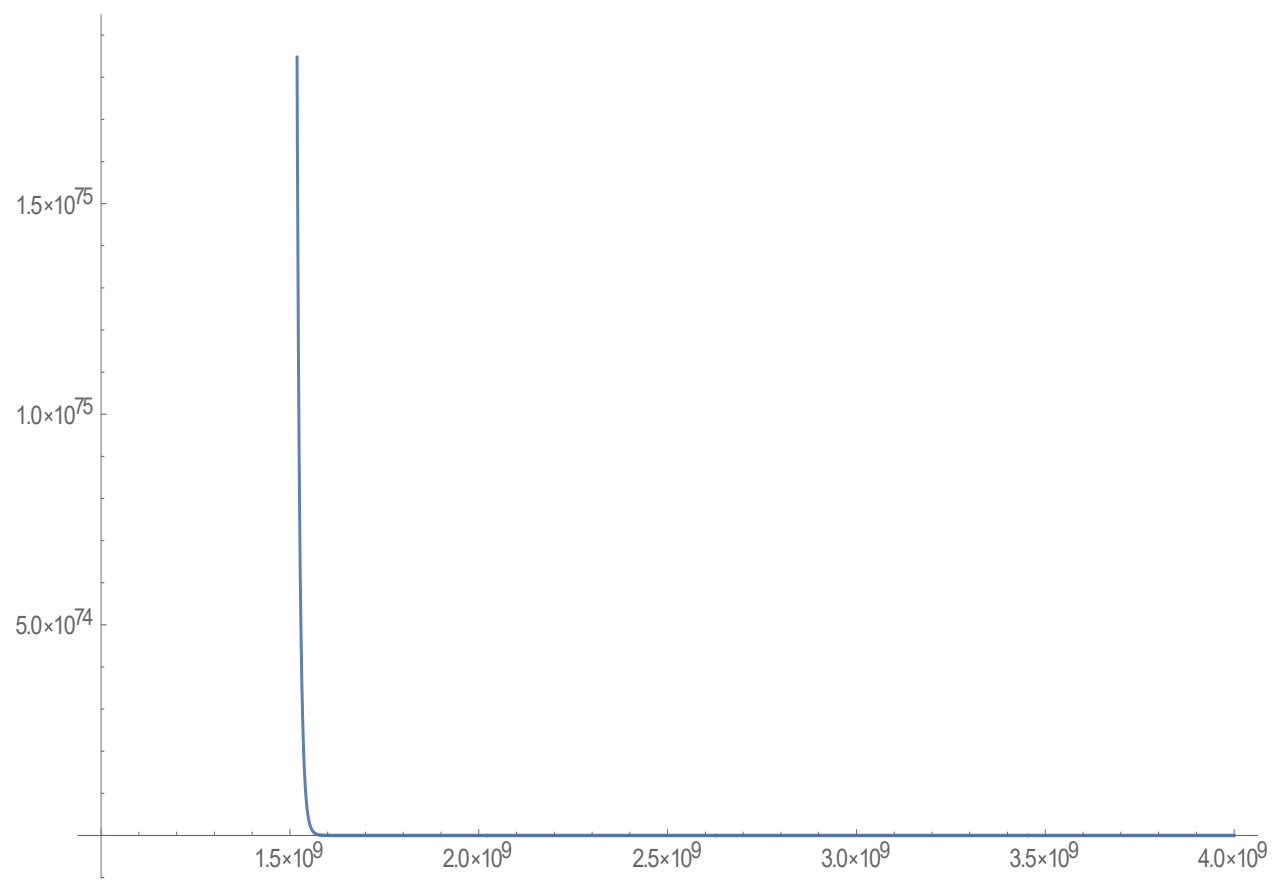

Fig. 16. PlotGraph of the Electric Field Intensity $f(r)$ (vertical axis) for the region $10^{9}<r<41^{9}$ (horizontal axis) in which the gravitational field acceleration has been chosen accordingly an electromagnetic mass of $10^{40}[\mathrm{~kg}]$ located at the center of the confinement, according Newton's Shell Theorem. And a corresponding Single Harmonic frequency of $0.2[\mathrm{~Hz}]$.

It follows from Figure 16 that the radius of the stable gravitational electromagnetic confinement of the Single Harmonic Black Hole SHBH equals approximately $1.510^{9}$ [m]. 


\subsection{Conclusions}

The inertia of Light is not comparable with the inertia of the mechanical mass of objects like the mechanical mass we measure in mechanical experiments. The mechanical mass (inertia) of objects is isotropic and the mechanical mass of a beam of light is an anisotropic property. The mechanical mass (inertia) of objects can be determined by Newton's second law of motion. It is impossible to accelerate or to decelerate a beam of light because the speed of light is a universal constant and for that reason it is impossible to define the mass of a beam of light in the direction of propagation with Newton's second law of motion.

However, the 2-Dimensional property of inertia related to the 2Dimensional confinement of a beam of light perpendicular to the direction of propagation can be accelerated or decelerated in the plane perpendicular to the directions of propagation like a mechanical object and fully obeys Newton's second law of motion in these directions. In a comparable way like a satellite propagates in a circular orbit around the earth, a beam of light propagates in a circular orbit around a black hole.

In the direction of propagation of a beam of light, the beam of light cannot be accelerated or decelerated because the speed of light is a universal constant. However, like a ball losing its kinetic energy (which will be transformed into potential energy) when a ball has been thrown up from the earth (in the direction of the gravitational field), a beam of light will lose its electromagnetic energy when it moves in the direction of a gravitational field away from a black hole. The opposite effect occurs when a beam of light moves towards a black hole, its electromagnetic energy will increase like the kinetic energy of a ball increases during its falls backwards to the earth.

In both examples the beam of light, inertia (mass) becomes an anisotropic property and behaves differently in the directions perpendicular to the direction of propagation compared to the direction of propagation.

Newton's law has become 4-Dimensional (equation (5)) and does not only represent classical mechanics but also represents classical electrodynamics, general relativity and quantum physics. Gravitational Electromagnetic Interaction results in the phenomenon of Clack Holes with specific mass, frequency, spin and electric charge. 


\subsection{Data Availability}

All the Data and all the Calculations to provide evidence to this 'New Theory about Light' have been published in the 'Open Source Framework(OSF)': https://osf.io/gbn4p/

DOI: 10.31219/osf.io/gbn4p ( https://doi.org/10.31219/osf.io/gbn4p)

(Calculations in Mathematica 11.0)', Page 1-33). 


\subsection{References}

[5] Li-Xin Li; A New Unified Theory of Electromagnetic and Gravitational Interactions, Frontiers of Physics, Volume 11, Issue 6, article id. 110402 (2016); arxiv.org/abs/1511.01260

[6] Richard Easther, Brian R Greene, Mark G Jackson and Daniel Kabat; String windings in the early universe, Journal of Cosmology and Astroparticle Physics, Volume 2005, February 2005

[7] J. Wheeler, Phys. Rev. 97, 511 (1955).

[8] Dirk Englund, Arka Majumdar, Michal Bajcsy, Andrei Faraon, Pierre Petroff, and Jelena Vučković; Ultrafast Photon-Photon Interaction in a Strongly Coupled Quantum Dot-Cavity System, Phys. Rev Lett. 108, 093604, March 2012, DOI : 10.1103/PhysRevLett.108.093604

[9] L. Filipe O. Costa, Georgios Lukes-Gerakopoulos, and Oldřich Semerák; Spinning particles in general relativity: Momentumvelocity relation for the Mathisson-Pirani spin condition; Phys. Rev. D 97, 084023 - Published 16 April 2018

[10] Ryotaro Kase, Masato Minamitsuji, and Shinji Tsujikawa; Relativistic stars in vector-tensor theories; Phys. Rev. D 97, 084009 - Published 9 April 2018

[11] Hector O. Silva, Jeremy Sakstein, Leonardo Gualtieri, Thomas P. Sotiriou, and Emanuele Berti; Spontaneous Scalarization of Black Holes and Compact Stars from a Gauss-Bonnet Coupling;Phys. Rev. Lett. 120, 131104 (2018) - Published 30 March 2018

[12] Jahed Abedi, Hannah Dykaar, and Niayesh Afshordi; Echoes from the abyss: Tentative evidence for Planck-scale structure at black hole horizons ;Phys. Rev. D 96, 082004 (2017) - Published 26 October 2017

[13] A. Hees, T. Do, A. M. Ghez, G. D. Martinez, S. Naoz, E. E. Becklin, A. Boehle, S. Chappell, D. Chu, A. Dehghanfar, K. Kosmo, J. R. Lu, K. Matthews, M. R. Morris, S. Sakai, R. Schödel, and G. Witzel; Testing General Relativity with Stellar Orbits around the Supermassive Black Hole in Our Galactic Center; Phys. Rev. Lett. 118, 211101 (2017) - Published 25 May 2017 
[14] J. W. Vegt, A Continuous Model of Matter based on AEONs, Physics Essays ,1995, Volume 8, Number 2, 201-224 A Continuous model of Matter ( https://doi.org/10.31219/osf.io/ra7ng )

[15] J. W. Vegt, Unified 4-Dimensional Hyperspace Equilibrium beyond Einstein 4-Dimensional, Kaluza-Klein 5-Dimensional and Superstring 10- and 11 Dimensional Curved Hyperspaces ( https://doi.org/10.31219/osf.io/vq2a4 )

[16] J. M. Maldacena, Black Holes in String Theory, Princeton University, arxiv.org/abs/hep-th/960723533.

[17] V. C. de Andrade and J. G. Pereira, Gravitational Lorentz force and the description of the gravitational interaction, Phys. Rev. D 56, 468

[18] Volodymyr Krasnoholovets, Motion of a Relativistic Particle and the Vacuum, Physics Essays, vol 10, no 3, 1997, 407-416, arXiv:quant-ph/9903077

[19] Donald H Kobe ; Quantum power in de Broglie-Bohm theory ; Journal of Physics A: Mathematical and Theoretical, Volume 40 Number 19 , Published 24 April 2007

[20] J. W. Vegt. Origin of "De Broglie Waves" (Calculations in Mathematica 11.0 in PDF format) Publisher Wolfram. https://doi.org/10.31219/osf.io/gbn4p

[21] J.W. Vegt. Calculations in Mathematica. Solutions for an electromagnetic field under the influence of a longitudinal gravitational field. 


\section{THE WORLD BEYOND BLACK HOLES LEVEL 3}

Light contains the key to open the doors to Heaven. Unfortunately, the same key fits on the doors to Hell

$\begin{array}{ll}\text { Author: } & \text { Wim Vegt } \\ \text { Country: } & \text { The Netherlands } \\ \text { Website: } & \text { https://wimvegt.topworld.center } \\ \text { Email: } & \text { j.w.vegt@topacademy.center }\end{array}$




\section{Classical Theory Newton}

NEWTON in 3 dimensions

$$
\begin{array}{r}
\overline{\mathrm{F}}=\mathrm{m} \overline{\mathrm{a}} \\
\left(\begin{array}{l}
\mathrm{x}_{3} \\
\mathrm{x}_{2} \\
\mathrm{x}_{1}
\end{array}\right) \rightarrow\left(\begin{array}{l}
\mathrm{F}_{\mathrm{z}} \\
\mathrm{F}_{\mathrm{y}} \\
\mathrm{F}_{\mathrm{x}}
\end{array}\right)=\mathrm{m}\left(\begin{array}{l}
\mathrm{a}_{\mathrm{z}} \\
\mathrm{a}_{\mathrm{y}} \\
\mathrm{a}_{\mathrm{x}}
\end{array}\right)
\end{array}
$$

New Theory

Newton

NEWTON in 4 dimensions

$$
\begin{aligned}
\overline{\mathrm{F}}^{4} & =\mathrm{m} \frac{-4}{\mathrm{a}^{-4}} \\
\left(\begin{array}{l}
\mathrm{x}_{4} \\
\mathrm{x}_{3} \\
\mathrm{x}_{2} \\
\mathrm{x}_{1}
\end{array}\right) \rightarrow\left(\begin{array}{l}
\mathrm{F}_{\mathrm{t}} \\
\mathrm{F}_{\mathrm{z}} \\
\mathrm{F}_{\mathrm{y}} \\
\mathrm{F}_{\mathrm{x}}
\end{array}\right) & =\mathrm{m}\left(\begin{array}{l}
\mathrm{a}_{\mathrm{t}} \\
\mathrm{a}_{\mathrm{z}} \\
\mathrm{a}_{\mathrm{y}} \\
\mathrm{a}_{\mathrm{x}}
\end{array}\right)
\end{aligned}
$$

Maxwell (Newton in 3 Dimensions)

$$
\begin{aligned}
& \nabla \cdot \overline{\mathrm{E}}=\frac{\rho}{\varepsilon} \\
& \nabla \times \overline{\mathrm{E}}=-\mu \frac{\partial \overline{\mathrm{H}}}{\partial \mathrm{t}} \\
& \nabla \cdot \overline{\mathrm{H}}=0 \\
& \nabla \times \overline{\mathrm{H}}=\varepsilon \frac{\partial \overline{\mathrm{E}}}{\partial \mathrm{t}}
\end{aligned}
$$

Dirac/ Schrôdinger (Newton $4^{\text {th }}$ Dimension)

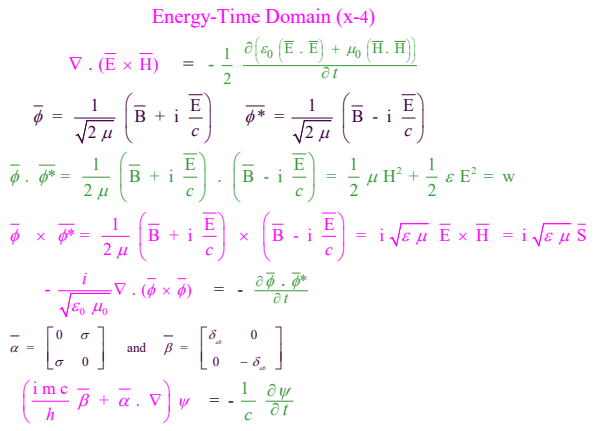

$$
\begin{aligned}
& \text { NEWTON: } \rightarrow \overline{\mathrm{F}}_{\text {TOTAL }}=\mathrm{m} \overline{\mathrm{a}}[\mathrm{N}] \rightarrow \overline{\mathrm{f}}_{\text {TOTAL }}=\rho \overline{\mathrm{a}}\left[\mathrm{N} / \mathrm{m}^{3}\right]
\end{aligned}
$$

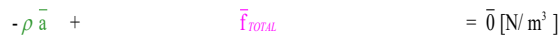

$$
\begin{aligned}
& -\rho \overline{\mathrm{a}}+\overline{\mathrm{f}}_{\text {HeCtric }}+\overline{\mathrm{f}}_{\text {MManetic }}=\overline{0}\left[\mathrm{~N} / \mathrm{m}^{3}\right]
\end{aligned}
$$

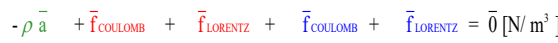

$$
\begin{aligned}
& -\frac{1}{c^{2}} \frac{\partial(\mathrm{E} \times \mathrm{H})}{\partial t}+\varepsilon_{0} \overline{\mathrm{E}}(\nabla, \overline{\mathrm{E}})-\varepsilon_{0} \overline{\mathrm{E}}_{\times}\left(\nabla \times \overline{\mathrm{E}}_{)}+\mu_{0} \overline{\mathrm{H}}\left(\nabla \cdot \overline{\mathrm{H}}_{)}-\mu_{0} \overline{\mathrm{H}}_{\times}(\nabla \times \overline{\mathrm{H}})=0\left[\mathrm{~N} / \mathrm{m}^{3}\right]\right.\right.
\end{aligned}
$$

Dirac/ Schrôdinger (Newton $4^{\text {th }}$ Dimension)

$$
\begin{aligned}
& \text { Energy-Time Domain (x-4) } \\
& \text { B-6 B-7 } \\
& \nabla \cdot(\overline{\mathrm{E}} \times \overline{\mathrm{H}})=-\frac{1}{2} \frac{\partial\left(\varepsilon_{0}(\overline{\mathrm{E}} \cdot \overline{\mathrm{E}})+\mu_{0}(\overline{\mathrm{H}} \cdot \overline{\mathrm{H}})\right)}{\partial t} \\
& \text { 3-Dimensional Space Domain } \\
& \text { B-1 B-2 B-3 } \\
& \left(\begin{array}{l}
x_{3} \\
x_{2} \\
x_{1}
\end{array}\right) \\
& \begin{array}{c}
-\frac{1}{c^{2}} \frac{\partial(\overline{\mathrm{E}} \times \overline{\mathrm{H}})}{\partial t}+\varepsilon_{0} \overline{\mathrm{E}}(\nabla \cdot \overline{\mathrm{E}})-\varepsilon_{0} \overline{\mathrm{E}} \times(\nabla \times \overline{\mathrm{E}})+ \\
+\mu_{0} \overline{\mathrm{H}}(\nabla \cdot \overline{\mathrm{H}})-\mu_{0} \overline{\mathrm{H}} \times(\nabla \times \overline{\mathrm{H}})=\overline{0}
\end{array}
\end{aligned}
$$

Quantum Gravity (Newton $4^{\text {th }}$ Dimension)

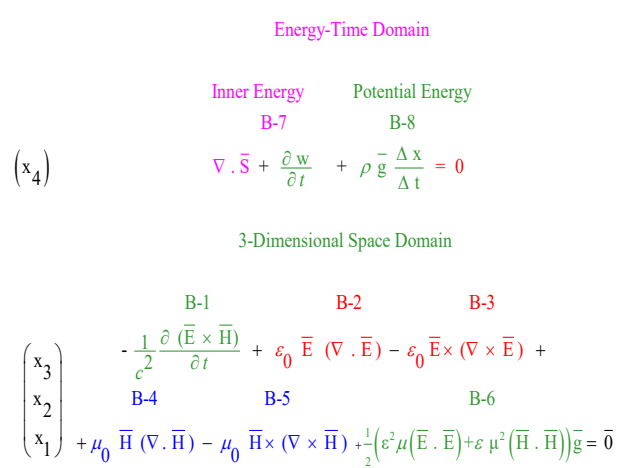




\section{Index}

3.1 Introduction $\underline{142}$

3.2 The 4th term in the Unified 4-Dimensional $\underline{150}$ Hyperspace Equilibrium Equation

3.3 The Impact of Gravity on Light 153

3.4 The Electromagnetic Perfect Equilibrium $\underline{160}$

3.5 EM Radiation within a Cartesian Coordinate $\underline{167}$ System in the absence of gravity

3.6 Laser Beam with a Gaussian division in the x-y $\underline{169}$ plane within a Cartesian Coordinate System in the absence of Gravity

3.7 EM Radiation within a Cartesian Coordinate System under the influence of a Longitudinal Gravitational Field g

3.8 The Real Light Intensity of the Sun, measured in $\quad \underline{175}$ our Solar System, including Electromagnetic Gravitational Conversion (EMGC)

3.9 The Boundaries of our Universe

3.10 The Origin of Dark Matter

3.11 Electromagnetic Radiation within a Spherical $\underline{184}$ Coordinate System

3.12 Confined Electromagnetic Radiation within a $\underline{186}$ Spherical Coordinate System through Electromagnetic-Gravitational Interaction

3.13 The fundamental conflict between Causality and 192 Probability

3.14 Confined Electromagnetic Radiation within a 195 Toroidal Coordinate System

3.15 Confined Electromagnetic Radiation within a 198 Toroidal Coordinate System through Electromagnetic-Gravitational Interaction in a non-linear Space-Time Continuum

3.16 The Origin of Electromagnetic Mass (Inertia) 
3.17 The radiation pressure on Mirror A, when Mirror 204 A moves with a velocity $\mathrm{v}$ in the direction of the positive z-axis

3.18 The radiation pressure on Mirror B when Mirror

$\underline{205}$

$B$ moves with a velocity $\mathrm{v}$ in the direction of the positive z-axis

3.19 Newton's second Law of Motion (Inertia) for

$\underline{207}$ Confined Electromagnetic Radiation

3.20 Quantum Mechanical Entanglement

3.21 A Gravitational-Electromagnetic Model beyond the Superstring

3.22 A Gravitational-Electromagnetic Confinement Type 1

$$
\left(\mathrm{emm}=10^{-4}[\mathrm{~kg}] \text {; radius }=2 \times 10^{-35}[\mathrm{~m}]\right) \text { : }
$$

3.23 A Gravitational-Electromagnetic Confinement Type 2

$$
\left(\mathrm{emm}=10^{-12}[\mathrm{~kg}] \text {; radius }=2.5 \times 10^{-43}[\mathrm{~m}]\right) \text { : }
$$

3.24 A Gravitational-Electromagnetic Confinement

Type $3\left(\mathrm{emm}=1.6726 \times 10^{-27}[\mathrm{~kg}]\right.$; radius $=3 \mathrm{x}$ $\left.10^{-58}[\mathrm{~m}]\right)$ :

3.25 The Origin of Electric Charge and Magnetic Spin in discrete values

3.26 The 5 Types of "Sub Max Planck Length Gravitational-Electromagnetic Confinements" resulting in Electric Charge and Magnetic Spin

3.26.1 Type 1 of "Sub Max Planck Length

Gravitational-Electromagnetic Confinements" (Electric- and Magnetic Dipoles, Electric- and Magnetic Spin) $\{\mathrm{m} 1=0, \mathrm{n} 1=0, \mathrm{p} 1=0, \mathrm{q} 1=0\}$

3.26.2 Type 2 of "Sub Max Planck Length Gravitational-Electromagnetic Confinements" (Electric- and Magnetic Dipoles, Electric- and Magnetic Spin) $\{\mathrm{m} 1=1, \mathrm{n} 1=0, \mathrm{p} 1=0, \mathrm{q} 1=0\}$ 
3.26.3 Type 3 of "Sub Max Planck Length

Gravitational-Electromagnetic Confinements" $\{\mathrm{m} 1=1, \mathrm{n} 1=1, \mathrm{p} 1=0, \mathrm{q} 1=0\}$

3.26.4 Type 4 of "Sub Max Planck Length

Gravitational-Electromagnetic Confinements" (Electric- and Magnetic Dipoles, Electric- and Magnetic Spin) $\{\mathrm{m} 1=0, \mathrm{n} 1=0, \mathrm{p} 1=1, \mathrm{q} 1=0\}$

3.26.5 Type 5 of "Sub Max Planck Length

Gravitational-Electromagnetic Confinements" $\{\mathrm{m} 1=0, \mathrm{n} 1=0, \mathrm{p} 1=1, \mathrm{q} 1=1\}$

3.27 Type II of "Sub Max Planck Length

Gravitational-Electromagnetic Confinements"

3.28 Concluding Remarks

3.29 Three fundamental Equations in Quantum Physics

3.30 Acknowledgement

3.31 Data Availability

3.32 References 


\section{$3 \quad$ Black Holes (level 3)}

\subsection{Introduction}

Albert Einstein, Lorentz and Minkowski published in 1905 the Theory of Special Relativity and Einstein published in 1915 his field theory of general relativity based on a curved 4-dimensional space-time continuum to integrate the gravitational field and the electromagnetic field in one unified field. Since then the method of Einstein's unifying field theory has been developed by many others in more than 4 dimensions resulting finally in the well-known 10dimensional and 11-dimensional "string theory".

String theory is an outgrowth of S-matrix theory, a research program begun by Werner Heisenberg in 1943 (following John Archibald Wheeler' ${ }^{(3)} 1937$ introduction of the S-matrix), picked up and advocated by many prominent theorists starting in the late 1950's.

Theodor Franz Eduard Kaluza (1885-1954), was a German mathematician and physicist well-known for the Kaluza-Klein theory involving field equations in curved five-dimensional space. His idea that fundamental forces can be unified by introducing additional dimensions re-emerged much later in the "String Theory".

The original Kaluza-Klein theory was one of the first attempts to create an unified field theory i.e. the theory, which would unify all the forces under one fundamental law. It was published in 1921 by Theodor Kaluza and extended in 1926 
by Oskar Klein. The basic idea of this theory was to postulate one extra compactified space dimension and introduce nothing but pure gravity in a new $(1+4)$-dimensional spacetime. Klein suggested that the fifth dimension would be rolled up into a tiny, compact loop on the order of $10^{-35}[\mathrm{~m}]$

To use simple notifications, the Einstein convention will be used. In the Einstein Convention the index always changes from 1 till 4 . Others prefer the changing of the index from 0 till 3 but is has the same meaning.

This means the term: " $\mathrm{x}_{\mathrm{a}} \mathrm{y}_{\mathrm{a}}$ " has to be interpreted in the basic equation (B-1) as:

$$
\mathrm{x}_{\mathrm{a}} \mathrm{y}_{\mathrm{a}}=\mathrm{x}_{1} \mathrm{y}_{1}+\mathrm{x}_{2} \mathrm{y}_{2}+\mathrm{x}_{3} \mathrm{y}_{3}+\mathrm{x}_{4} \mathrm{y}_{4}
$$

In Cartesian coordinates this means:

$$
(1,2,3,4) \rightarrow(\mathrm{x}, \mathrm{y}, \mathrm{z}, \mathrm{t}) \quad \text { or } \quad(0,1,2,3) \rightarrow(\mathrm{t}, \mathrm{x}, \mathrm{y}, \mathrm{z})
$$

In Classical Electrodynamics, the Electromagnetic Field has been derived from the 4-dimensional Potential 4-Vector. The 4-dimensional Electromagnetic "Potential 4-vector", oriented in the classical complex 4-dimensional "Minkowski Space" will be defined by $\varphi_{a}$ ("a" varying from 1 until 4) in which:

$$
\varphi_{a}=\left(\begin{array}{c}
\mathrm{i} \mathrm{V} / \mathrm{c} \\
\mathrm{A}_{3} \\
\mathrm{~A}_{2} \\
\mathrm{~A}_{1}
\end{array}\right)
$$


In which $\mathrm{V}$ equals the scalar electric potential and $\overline{\mathrm{A}}$ the 3dimensional magnetic vector potential.

In which $\mathrm{V}$ equals the scalar electric potential and $\overline{\mathrm{A}}$ the 3dimensional magnetic vector potential. The Electric Field Intensity $\overline{\mathrm{E}}$ has been represented in the basic equation (B-4):

$$
\overline{\mathrm{E}}=-\nabla \mathrm{V}-\frac{\partial \overline{\mathrm{A}}}{\partial t}
$$

The Magnetic Field Intensity $\overline{\mathrm{H}}$ has been represented in the basic equation (B-5):

$$
\overline{\mathrm{H}}=\frac{1}{\mu_{0}} \mathrm{~B}=\frac{1}{\mu_{0}}(\nabla \times \overline{\mathrm{A}})
$$

The 4-dimensional Electromagnetic "Maxwell Tensor" has been defined by:

$$
\mathrm{F}_{a b}=\partial_{b} \varphi_{a}-\partial_{a} \varphi_{b}
$$

The 4-dimensional Electromagnetic "Energy Momentum Tensor" has been defined by:

$$
T^{a b}=\frac{1}{\mu_{0}}\left[F_{a c} F^{c b}+\frac{1}{4} \delta_{a b} F_{c d} F^{c d}\right]
$$


The 4-dimensional divergence of the Energy Momentum Tensor equals the 4-dimensional Force Density 4-vector:

$$
f^{a}=\partial_{b} \mathrm{~T}^{a b}
$$

The new theory has been based on the fundamental concept of Harmony in which all force densities in the Universe have been counter balanced by equal and opposite directed force densities resulting in a net force density equals zero and a final set of 4 Electromagnetic Equations.

The meet the Universal 4-dimensional equilibrium requirements in space and in time, equation (3) transfers into the Universal Fundamental Equilibrium Equation (4) in which the divergence of the Energy Momentum Tensor equals zero. (the 4-dimensional Force Density 4-vector equals the 4 -vector zero):

$$
f^{a}=\partial_{b} \mathrm{~T}^{a b}=0
$$

In the absence of any Gravity, the force density $f^{a}$ in the 3 directions of the 3 coordinates of the chosen 3-coordinate system follows from the (4-dimensional) Divergence of the (4-dimensional) Stress Energy Tensor ${ }^{(8,9,38)}$ (3).

The Divergence of a Vector equals a Scalar. The Divergence of a Tensor equals a Vector. The 4-dimensional Divergence of the 4-dimensional Stress Energy Tensor (4) equals the 4dimensional Force-density Vector $\mathrm{f}^{\mathrm{a}}$.

Equation (4) represents the foundation for 5 famous equations. The 3 -dimensional representation of (4) 
represents Newton's well-known law of motion $(\mathrm{F}=\mathrm{m} \mathrm{a})$. The 3 spatial dimensions of the 4-dimensional presentation of (4) represents an improved version of the Maxwell equations. The presentation of (4) in the $4^{\text {th }}$ dimension (time) represents the quantum mechanical Schrödinger Wave Equation and the relativistic Dirac Equation.

Introducing the gravitational field in (4) represents the foundation in Einstein's Theory of General Relativity.

$$
\begin{aligned}
& \text { (4.1) Newton ( F = m a) } \\
& \text { (4.2) Maxwell's } 4 \text { equations } \\
& f^{a}=\partial_{b} \mathrm{~T}^{a b}=0 \quad \text { (4.3) Schrödinger Wave Equation } \\
& \text { (4.4) Relativistic Dirac Equation } \\
& \text { (4.5) Einstein's Field Equations } \\
& \text { (General Relativity) }
\end{aligned}
$$

The first 3 terms of the 4-dimensional Force-density vector equal the force densities in the corresponding 3 dimensions of the chosen Coordinate System. The $4^{\text {th }}$ component equals the Electromagnetic Poynting's Theorhem (5.1) (Continuity Equation).

To calculate the equilibrium conditions to present the force densities in the Electromagnetic Field Configuration, the first 3 terms of the 4-dimensional Force-density vector are being used. 
By re-arranging the first 3 terms of the (4-dimensional Divergence) of the (4-dimensional) Stress Energy Tensor (4)

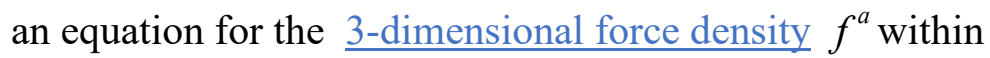
the Electromagnetic Field Configuration has been created. This Equation (5.2) represents the 3-dimensional force density $f^{a}$ in a coordinate-free vector equation in the absence of any Gravity:

$$
\begin{gathered}
\left(\mathrm{x}_{4}\right) \quad \nabla \cdot(\overline{\mathrm{E}} \times \overline{\mathrm{H}})+\frac{1}{2} \frac{\partial\left(\varepsilon_{0}(\overline{\mathrm{E}} \cdot \overline{\mathrm{E}})+\mu_{0}(\overline{\mathrm{H}} \cdot \overline{\mathrm{H}})\right)}{\partial t}=0 \\
\left(\begin{array}{l}
\mathrm{x}_{3} \\
\mathrm{x}_{2} \\
\mathrm{x}_{1}
\end{array}\right) \quad-\frac{1}{c^{2}} \frac{\partial(\overline{\mathrm{E}} \times \overline{\mathrm{H}})}{\partial t}+\varepsilon_{0} \overline{\mathrm{E}}(\nabla \cdot \overline{\mathrm{E}})-\varepsilon_{0} \overline{\mathrm{E}} \times(\nabla \times \overline{\mathrm{E}})+ \\
+\mu_{0} \overline{\mathrm{H}}(\nabla \cdot \overline{\mathrm{H}})-\mu_{0} \overline{\mathrm{H}} \times(\nabla \times \overline{\mathrm{H}})=\overline{0}
\end{gathered}
$$

According the fundamental requirement of a perfect equilibrium at any place at any time in any direction, the algebraic sum of all the different force densities $\bar{f}$ have to be equal zero for any physical possible electromagnetic field configuration (particles and fields).

It is fundamental to realize that 3 different kind of separate interactions of the force densities in (5.2) are being involved within this resulting equilibrium. Magnetic-Magnetic interaction ( $4^{\text {th }}$ and $5^{\text {th }}$ term in 5.2), Electric-Electric interaction $\left(2^{\text {nd }}\right.$ and $3^{\text {rd }}$ term in 5.2), Electric-Magnetic 147 
interaction and reverse ( $1 \mathrm{st}$ term in 5.2) which is timedependent according the theory of special relativity.

An electromagnetic field which is in a perfect equilibrium with itself and its surrounding at any space and time in any direction, fulfills the necessary requirements for the physical possibility of the existence of this field. Under that condition Equation (4) transforms into the "Unified 4-Dimensional Hyperspace Equilibrium Equation” (5)

$$
\begin{aligned}
& -\frac{1}{c^{2}} \frac{\partial(\overline{\mathrm{E}} \times \overline{\mathrm{H}})}{\partial t}+\varepsilon_{0} \overline{\mathrm{E}}(\nabla . \overline{\mathrm{E}})-\varepsilon_{0} \overline{\mathrm{E}} \times(\nabla \times \overline{\mathrm{E}})+ \\
& +\mu_{0} \overline{\mathrm{H}}(\nabla . \overline{\mathrm{H}})-\mu_{0} \overline{\mathrm{H}} \times(\nabla \times \overline{\mathrm{H}})=\overline{0}
\end{aligned}
$$

To extend Field Equation (5) into an equilibrium within a multi-dimensional curved Space-Time continuum unifying different fields like gravity and electromagnetism, the transformation has been realized by the transformation of the resulting force-densities within the 4-Dimensional SpaceTime continuum. The Unification of the Electromagnetic Fields with the Gravitational fields results in the Relativistic Gravitational Electro Magnetic Equilibrium (RGEE) equation within a gravitational field $\bar{g}$ in the 3 -dimensional (spatial) representation:

$$
\begin{gathered}
-\frac{1}{c^{2}} \frac{\partial(\bar{E} \times \bar{H})}{\partial t}+\varepsilon_{0} \bar{E}(\nabla \cdot \bar{E})-\varepsilon_{0} \bar{E} \times(\nabla \times \bar{E})+\mu_{0} \bar{H}(\nabla \cdot \bar{H})- \\
-\mu_{0} \bar{H} \times(\nabla \times \bar{H})-\frac{1}{2} \varepsilon_{0}^{2} \mu_{0}(\bar{E} \cdot \bar{E}) \overline{\mathrm{g}}-\frac{1}{2} \varepsilon_{0} \mu_{0}^{2}(\bar{H} \cdot \bar{H}) \overline{\mathrm{g}}=\overline{0}
\end{gathered}
$$

It is fundamental to realize that Equation (5) is only a part of the 4-Dimensional Time-Space Continuum Equation. 
The Divergence of the 4-Dimensional Stress-Energy Tensor (3) results in the 4-Dimensional Vector Equation. The first 3 terms of the vector Equation have been presented in (5). The $4^{\text {th }}$ term presents the Continuum Equation. By introducing the complex field notations for the electric field and the magnetic field in Equation (39) the $4^{\text {th }}$ term transforms into the wellknown relativistic quantum mechanical Dirac Equation and at low velocities into the quantum mechanical Schrödinger Wave Equation.

The 4 Equations together (3 Equations for the separate space coordinates) and the Dirac/Schrödinger Equation describe the Unification in a perfect Equilibrium of the different Fields. 


\subsection{The $4^{\text {th }}$ term in the Unified 4-Dimensional Hyperspace Equilibrium Equation}

The 4-Dimensional Hyperspace Equilibrium Dirac Equation (5.1) in the absence of gravity equals:

$$
\begin{gathered}
\left(\mathrm{x}_{4}\right) \quad \nabla \cdot(\overline{\mathrm{E}} \times \overline{\mathrm{H}})=-\frac{1}{2} \frac{\partial\left(\varepsilon_{0}(\overline{\mathrm{E}} \cdot \overline{\mathrm{E}})+\mu_{0}(\overline{\mathrm{H}} \cdot \overline{\mathrm{H}})\right)}{\partial t} \\
\left(\begin{array}{c}
\mathrm{x}_{3} \\
\mathrm{x}_{2} \\
\mathrm{x}_{1}
\end{array}\right) \begin{array}{c}
-\frac{1}{c^{2}} \frac{\partial(\overline{\mathrm{E}} \times \overline{\mathrm{H}})}{\partial t}+\varepsilon_{0} \overline{\mathrm{E}}(\nabla \cdot \overline{\mathrm{E}})-\varepsilon_{0} \overline{\mathrm{E}} \times(\nabla \times \overline{\mathrm{E}})+ \\
+\mu_{0} \overline{\mathrm{H}}(\nabla \cdot \overline{\mathrm{H}})-\mu_{0} \overline{\mathrm{H}} \times(\nabla \times \overline{\mathrm{H}})=\overline{0}
\end{array}
\end{gathered}
$$

The Poynting Theorem (5.1) can be rewritten by introducing the vector functions $\bar{\phi}$ and the complex conjugated vector function $\bar{\phi}^{*}$ in which:

$$
\bar{\phi}=\frac{1}{\sqrt{2 \mu}}\left(\overline{\mathrm{B}}+\mathrm{i} \frac{\overline{\mathrm{E}}}{\mathrm{c}}\right)
$$

$\overline{\mathrm{B}}$ equals the magnetic induction, $\overline{\mathrm{E}}$ the electric field intensity and $\mathrm{c}$ the speed of light. The complex conjugated vector function equals:

$$
\overline{\phi^{*}}=\frac{1}{\sqrt{2 \mu}}\left(\overline{\mathrm{B}}-\mathrm{i} \frac{\overline{\mathrm{E}}}{\mathrm{c}}\right)
$$


The dot product equals the electromagnetic energy density w:

$\bar{\phi} \cdot \bar{\phi}^{*}=\frac{1}{2 \mu}\left(\overline{\mathrm{B}}+\mathrm{i} \frac{\overline{\mathrm{E}}}{\mathrm{c}}\right) \cdot\left(\overline{\mathrm{B}}-\mathrm{i} \frac{\overline{\mathrm{E}}}{\mathrm{c}}\right)=\frac{1}{2} \mu \mathrm{H}^{2}+\frac{1}{2} \varepsilon \mathrm{E}^{2}=\mathrm{w}$

The cross product is proportional to the Poynting vector (ref. 29, page 202, equation 15).

$\bar{\phi} \times \overline{\phi^{*}}=\frac{1}{2 \mu}\left(\overline{\mathrm{B}}+\mathrm{i} \frac{\overline{\mathrm{E}}}{\mathrm{c}}\right) \times\left(\overline{\mathrm{B}}-\mathrm{i} \frac{\overline{\mathrm{E}}}{\mathrm{c}}\right)=\mathrm{i} \sqrt{\varepsilon \mu} \overline{\mathrm{E}} \times \overline{\mathrm{H}}=\mathrm{i} \sqrt{\varepsilon \mu} \overline{\mathrm{S}}$

Substituting (5.1.3) and (5.1.4) in Equation (5.1) results in The 4-Dimensional Hyperspace Equilibrium Equation (5.1.5):

$$
\begin{gathered}
\left(\mathrm{x}_{4}\right)-\frac{i}{\sqrt{\varepsilon_{0} \mu_{0}}} \nabla \cdot(\bar{\phi} \times \bar{\phi})=-\frac{\partial \bar{\phi} \cdot \bar{\phi}^{*}}{\partial t} \\
\left(\begin{array}{c}
\mathrm{x}_{3} \\
\mathrm{x}_{2} \\
\mathrm{x}_{1}
\end{array}\right) \begin{array}{c}
-\frac{1}{c^{2}} \frac{\partial(\overline{\mathrm{E}} \times \overline{\mathrm{H}})}{\partial t}+\varepsilon_{0} \overline{\mathrm{E}}(\nabla \cdot \overline{\mathrm{E}})-\varepsilon_{0} \overline{\mathrm{E}} \times(\nabla \times \overline{\mathrm{E}})+ \\
+\mu_{0} \overline{\mathrm{H}}(\nabla \cdot \overline{\mathrm{H}})-\mu_{0} \overline{\mathrm{H}} \times(\nabla \times \overline{\mathrm{H}})=\overline{0}
\end{array}
\end{gathered}
$$

To transform the electromagnetic vector wave function $\bar{\phi}$ into a scalar (spinor or one-dimensional matrix representation), the Pauli spin matrices $\sigma$ and the following matrices (Ref. 29 page 213, equation 99 ) are introduced:

$$
\bar{\alpha}=\left[\begin{array}{ll}
0 & \sigma \\
\sigma & 0
\end{array}\right] \quad \text { and } \quad \bar{\beta}=\left[\begin{array}{cc}
\delta_{a b} & 0 \\
0 & -\delta_{a b}
\end{array}\right]
$$


Then equation (5) can be written as the 4-Dimensional Hyperspace Equilibrium Dirac Equation:

$$
\begin{gathered}
\left(\mathrm{x}_{4}\right) \quad\left(\frac{\mathrm{imc} \bar{\beta}+\bar{\alpha} \cdot \nabla) \psi=-\frac{1}{c} \frac{\partial \psi}{\partial t}}{h}\right. \\
\left(\begin{array}{l}
\mathrm{x}_{3} \\
\mathrm{x}_{2} \\
\mathrm{x}_{1}
\end{array}\right) \quad \begin{array}{c}
-\frac{1}{c^{2}} \frac{\partial(\overline{\mathrm{E}} \times \overline{\mathrm{H}})}{\partial t}+\varepsilon_{0} \overline{\mathrm{E}}(\nabla \cdot \overline{\mathrm{E}})-\varepsilon_{0} \overline{\mathrm{E}} \times(\nabla \times \overline{\mathrm{E}})+ \\
+\mu_{0} \overline{\mathrm{H}}(\nabla \cdot \overline{\mathrm{H}})-\mu_{0} \overline{\mathrm{H}} \times(\nabla \times \overline{\mathrm{H}})=\overline{0}
\end{array}
\end{gathered}
$$

The fourth term $\left(\mathrm{x}_{4}\right)$ equals the relativistic Dirac equation (5.1.7) which equals equation (102) page 213 in Ref.29. 


\subsection{The Impact of Gravity on Light}

We consider a beam of light approaching a strong gravitational field. (E.g. a Black Hole). According the first term in equation (5.2) the beam of light will follow a circular orbit around the Black Hole. The required Equilibrium will exist at the radius where the centrifugal electromagnetic inertia forces will be equal and opposite directed to the centripetal oriented gravitational forces on the electromagnetic mass.

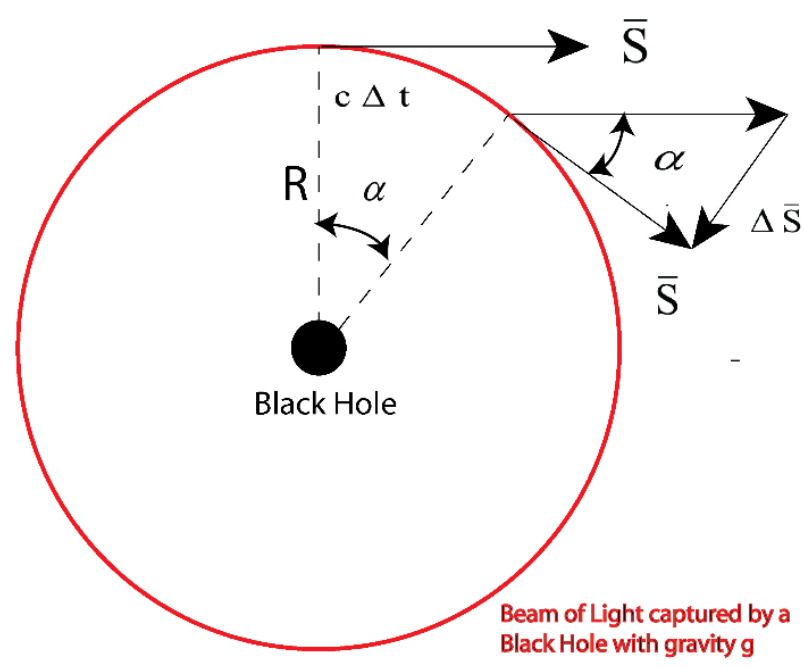

Fig. 1 A beam of light following a circular orbit around a Black Hole

The whole Universe is in a perfect Equilibrium. This fundamental property of Equilibrium has been extended to a 4-dimensional Hyperspace Continuum in which a perfect equilibrium persists in any of the 4 coordinate directions.

The requirement of a 4-dimensional Equilibrium results in the outcome that the Dirac Equation is only one equation in a set of 4 equations. And that the Dirac Equation originates 153 
from an electromagnetic equation in the time-energy domain. This new 4-Dimensional Hyperspace Equilibrium Theory opens a new door to an unexplored field of mathematical and physical challenges. This theory is a new approach in physics based on a 4-Dimensional Hyperspace Equilibrium resulting in the 4-dimensional Dirac Equation which represents the "Unification of (quantum mechanical) De Broglie Waves and Electromagnetic Waves". Solving these 4 simultaneous equations offers the possibility to find answers to the fundamental questions in physics within a quantum mechanical 4-Dimensional Framework. Every Physical Possible Electro-Magnetic Field Configuration of Confinement has to be a solution of the fundamental 4Dimensional Relativistic Dirac Equation (5).

$$
\begin{gathered}
\text { Energy- Time Domain } \\
\left(\mathrm{x}_{4}\right) \quad\left(\frac{\mathrm{i} \mathrm{m} \mathrm{c}}{h} \bar{\beta}+\bar{\alpha} \cdot \nabla\right) \psi=-\frac{1}{c} \frac{\partial \psi}{\partial t} \\
\text { 3-Dimensional Space Domain } \\
\mathrm{B}-1 \quad \text { B-2 } \\
\left(\begin{array}{c}
\mathrm{x}_{3} \\
\mathrm{x}_{2} \\
\mathrm{x}_{1}
\end{array}\right) \quad-\frac{1}{c^{2}} \quad \frac{\partial(\overline{\mathrm{E}} \times \overline{\mathrm{H}})}{\partial t}+\varepsilon_{0} \overline{\mathrm{E}}(\nabla \cdot \overline{\mathrm{E}})-\varepsilon_{0} \overline{\mathrm{E}} \times(\nabla \times \overline{\mathrm{E}})+ \\
+\mu_{0} \overline{\mathrm{H}}(\nabla \cdot \overline{\mathrm{H}})-\mu_{0} \overline{\mathrm{H}} \times(\nabla \times \overline{\mathrm{H}})=\overline{0}
\end{gathered}
$$

The Equation (5.1.7) in the Time-Energy Domain (x4) equals the well-known Quantum Mechanical Dirac Equation. The 3 Equations in the 3 spatial directions (coordinates $\mathrm{x} 1, \mathrm{x} 2, \mathrm{x} 3$ ) describe the Electromagnetic Field. The term B-1 controls the speed of light and represents the inertia (momentum) of a beam of light. The terms B-2 and B-3 control the confinement of Light for the Electric Radiation Pressure and the terms B-4 and B-5 control the confinement of Light for the Magnetic Radiation Pressure. 
As a demonstration of the power of the 4-dimensional Relativistic Dirac Equation, we observe a very basic and simple polarized laser beam with a Gaussian Intensity Division. The laser beam propagates towards the z-direction. The Electric Field "E" is oriented along the $\mathrm{x}$-axis and the Magnetic Field " $\mathrm{H}$ " is oriented along the y-axis.

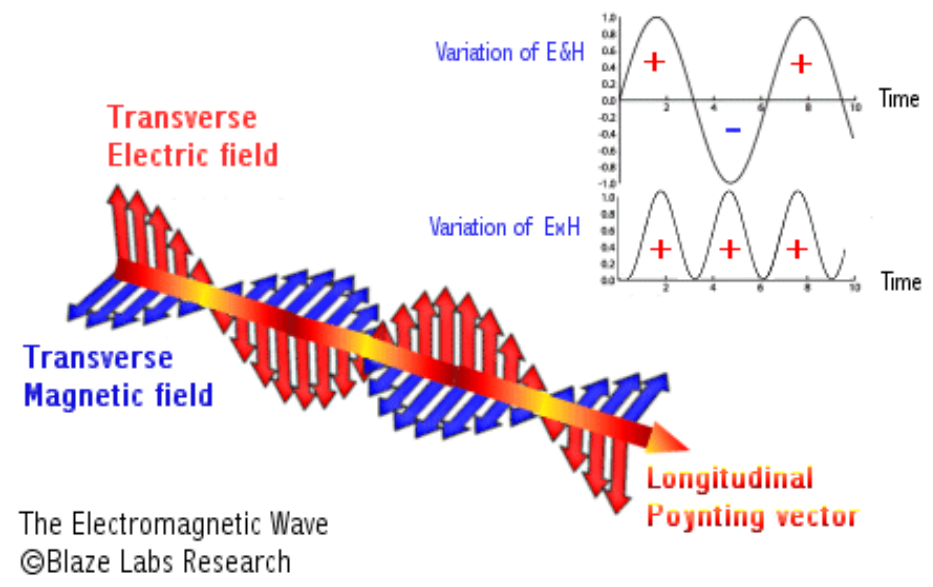

Fig. 2 Transversal Electromagnetic Wave

The laser beam propagates towards the positive z-direction:

Fig. 3 The Front Surface of a Laser Beam in which a perfect equilibrium does exist between the forward directed Radiation Pressure and the backwards directed Electromagnetic Inertia Forces 
The laser beam propagates towards the positive $\mathrm{z}$-direction with the speed of light. That is only possible because exactly at the speed of light there will exist a "Perfect Equilibrium" between the Electromagnetic Radiation Pressure towards the positive z-direction and the inertia term of the Electromagnetic Radiation Energy (electromagnetic mass) which has been represented by the time derivative of the "Poynting Vector" in term B-1 and is oriented along the negative $\mathrm{z}$-direction.

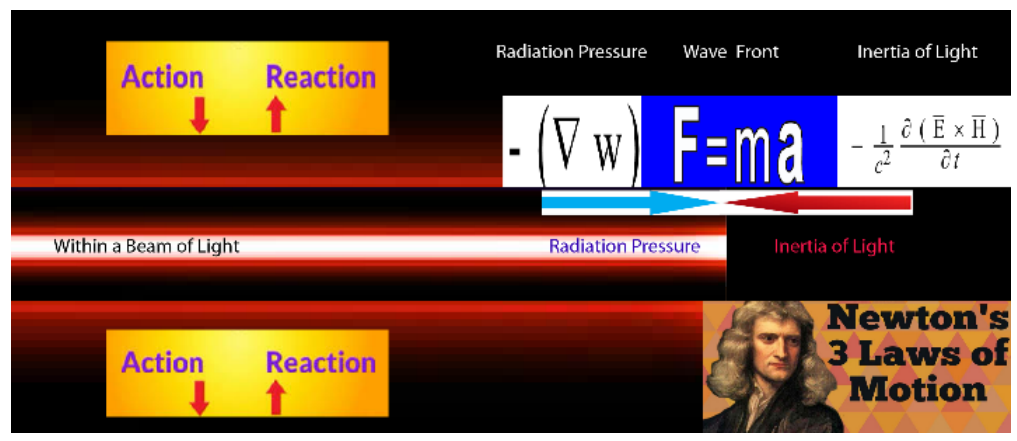

Fig. 4 Newton's second Law of Motion applied at the Front Surface of a Laser Beam. Exactly at the speed of light, a perfect equilibrium does exist between the forward directed Radiation Pressure $-\nabla$ w and the backwards directed Electromagnetic Inertia Force $-\frac{1}{c^{2}} \frac{\partial(\overline{\mathrm{E}} \times \overline{\mathrm{H}})}{\partial \mathrm{t}}$.

In the Unified 4-dimensional Equilibrium always a "Perfect Equilibrium" does exist between the Electromagnetic Radiation Pressure towards the positive z-direction and the inertia term of the Electromagnetic Radiation Energy (electromagnetic mass) which has been represented by the time derivative of the "Poynting Vector" in term B-1 and is oriented along the negative z-direction. 
The inward oriented Electromagnetic Force Density in the z-direction for every arbitrary function $\mathrm{f}(\mathrm{x}, \mathrm{y}, \mathrm{z})$ propagating with the speed of light $c=1 / \sqrt{\varepsilon_{0} \mu_{0}}$ :

$$
\mathrm{f}_{z}=-\varepsilon_{0} \mu_{0} \frac{\partial \overline{\mathrm{E}} \times \overline{\mathrm{H}}}{\partial \mathrm{t}}=-2 \varepsilon_{0}^{3 / 2} \sqrt{\mu_{0}} \mathrm{f}(\mathrm{x}, \mathrm{y})^{2} \mathrm{~g}\left(\mathrm{t}-\mathrm{z} \sqrt{\varepsilon_{0} \mu_{0}}\right) \mathrm{g}^{\prime}\left(\mathrm{t}-\mathrm{z} \sqrt{\varepsilon_{0} \mu_{0}}\right)
$$

Compensates the outward oriented Electromagnetic Radiation Pressure in the zdirection for every arbitrary function $\mathrm{f}(\mathrm{x}, \mathrm{y}, \mathrm{z})$ propagating with the speed of light $c=1 / \sqrt{\varepsilon_{0} \mu_{0}}$ :

$$
\mathrm{f}_{z}=-(\nabla \mathrm{w})_{z}=2 \varepsilon_{0}^{3 / 2} \sqrt{\mu_{0}} \mathrm{f}(\mathrm{x}, \mathrm{y})^{2} \mathrm{~g}\left(\mathrm{t}-\mathrm{z} \sqrt{\varepsilon_{0} \mu_{0}}\right) \mathrm{g}^{\prime}\left(\mathrm{t}-\mathrm{z} \sqrt{\varepsilon_{0} \mu_{0}}\right)
$$

The electromagnetic Inertia Force $"-\frac{1}{\mathrm{c}^{2}} \frac{\partial(\overline{\mathrm{E}} \times \overline{\mathrm{H}})}{\partial \mathrm{t}} "$

has been determined by the electromagnetic "Mass (Energy Density)" and the "Propagation Speed".

The electromagnetic "Radiation Pressure" in the positive zdirection (direction of propagation) has been determined by the electromagnetic Energy Density " w ".

There is only "One Exact Single Velocity" at which "Velocity" the electromagnetic "Radiation Pressure" in the in the positive z-direction perfectly counterbalances the opposite directed (negative z-direction) "Force of Inertia". That single speed has been called the "Velocity of Perfect Equilibrium" and has been called in general the "Speed of Light" and is independent of the frequency and the intensity of the electromagnetic beam of light.

$$
\text { Velocity of Perfect Equilibrium }=\mathrm{c}=\frac{1}{\sqrt{\varepsilon_{0} \mu_{0}}}=299,792[\mathrm{~km} / \mathrm{s}]
$$

When a laser beam (beam of light) passes a gravitational field with acceleration "g" in the $(\mathrm{x}, \mathrm{y})$ direction, the radiation 157 
pressures within de the beam of light in the $(\mathrm{x}, \mathrm{y})$ plane will shift due to gravitational forces.

Because according Einstein's $\mathrm{E}=\mathrm{m} \mathrm{c}^{2}$, the electromagnetic energy of the beam of light has electromagnetic mass, which will be attracted by the gravitational field. The shift in the radiation pressures in the $\mathrm{x}-\mathrm{y}$ plane due to gravitationalelectromagnetic interaction can only be compensated by the inertia forces within the $(\mathrm{x}, \mathrm{y})$ plane due to a circular orbit of the beam of light with the origin of the gravitational field as the center.

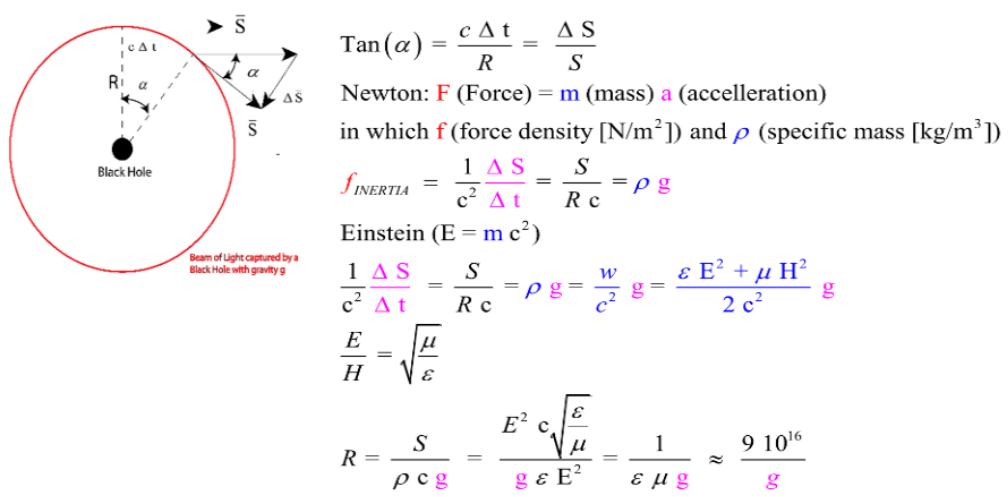

Fig. 5 Newton's second Law of Motion applied at the "Side Surface" of a Laser Beam. Exactly at the Radius " $R$ " of a circular orbit of a beam of light around a Black Hole, a perfect equilibrium does exist between the Radial outward directed Electromagnetic Inertia Force $-\frac{1}{c^{2}} \frac{\partial(\overline{\mathrm{E}} \times \overline{\mathrm{H}})}{\partial \mathrm{t}}$ and the Radial inward directed gravitational force density $\rho \overline{\mathrm{g}}$.

The perfect equilibrium, where the inertia forces due to the circular orbit of the beam of light are in a perfect balance with the attractive gravitational forces, exists at one defined radius " $R$ " of the beam of light (laser beam), independent of 
the intensity of the beam of light and independent of the frequency of the beam of light. Only the acceleration " $g$ " of the gravitational field determines the radius of equilibrium "R"

$$
R \approx \frac{910^{16}}{g}
$$

In which $\mathrm{R}$ the radius of the beam of light, and $\mathrm{g}$ the accelleration of the gravitational field of the Black Hole 


\subsection{The Electromagnetic Perfect Equilibrium}

A beam of light, the projection of a slide or a Laser Beam and in particular a single photon is an example of the "Perfect Physical Equilibrium" in which the outward oriented radiation pressure has been compensated completely by the oppositely oriented electromagnetic interaction and/or inertia forces.

The possibility of the existence of a beam of light, a Laser Beam, the projection of a slide on a screen or a single photon has never been understood with the theory of James Clerk Maxwell's Classical Electrodynamics.

According Classical Electrodynamics the speed of light always equals the physical constant "c" in any direction. Like a spherical electromagnetic wave. But for a Laser Beam, the speed of Light equals "c" in the direction of propagation but equals "zero" in the directions perpendicular to the direction of propagation. This unique phenomenon can only be explained by Newton's second law of motion applied to a Laser Beam. 


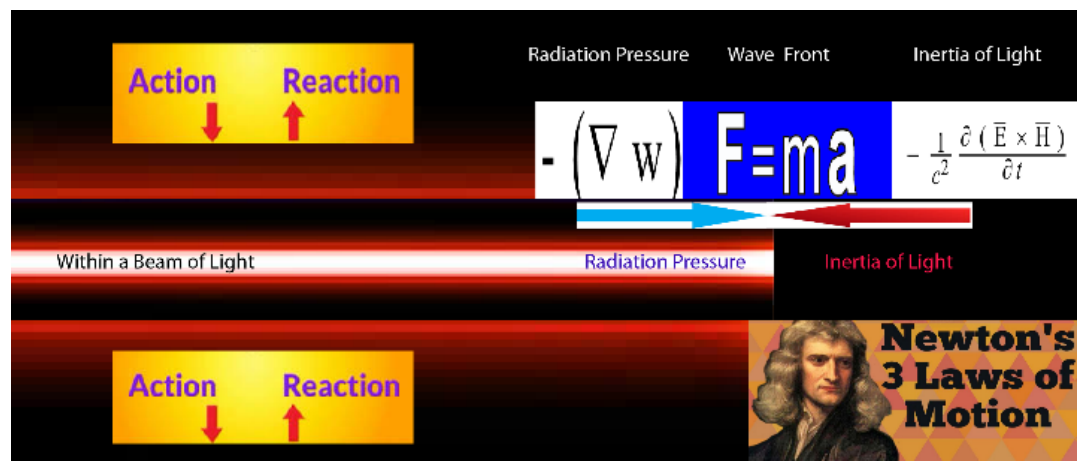

Fig. 6 Newton's second Law of Motion applied at the Front Surface of a Laser Beam propagating towards the z-direction.

As an example within a Cartesian Coordinate System (x,y,z), we choose for a Laser Beam propagating in the $\mathrm{z}$-direction while the speed of light in the $(x, y)$ direction equals zero.

The $x-y$ plane is oriented perpendicular on the z-direction. The speed of light towards the positive z-direction equals the speed of light (the constant "c = 299,792,458 m / s").

But the speed of light in the x-y plane has to be exactly zero. Else the diameter of the laser beam would become larger and larger during the propagation along the positive $\mathrm{z}$-direction.

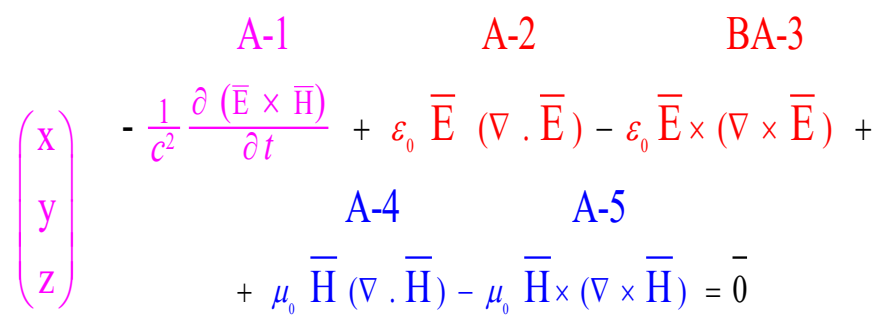


The only possible configuration for any kind of electromagnetic phenomenon (confinement of electromagnetic radiation) does exist in a configuration in which there is a perfect equilibrium between all the electromagnetic, gravitational and inertia forces.

For the surfaces perpendicular to the surface in the z-direction of propagation of a Laser Beam counts that the Electromagnetic confining forces A-2, A-3, A-4 and A-5 compensate exactly the outward oriented radiation pressure towards the $\mathrm{x}$-direction and the y-direction.

The Electric Radiation Pressure has been compensated by the Coulomb Force Densities within the Laser Beam

Coulomb's Law (B-2):

$$
\begin{array}{ll}
\overline{\mathrm{F}}=\overline{\mathrm{E}} \mathrm{Q} & (\mathrm{F} \triangleq \text { Force and } \mathrm{Q} \triangleq \text { Electric Charge }) \\
\overline{\mathrm{f}}=\overline{\mathrm{E}} \rho & (\mathrm{f} \triangleq \text { Force density and } \rho \hat{=} \text { Charge Density }) \\
\overline{\mathrm{f}}=\overline{\mathrm{E}} \nabla \cdot \overline{\mathrm{D}} & (\mathrm{D} \triangleq \text { Dielectric Displacement }) \\
\overline{\mathrm{f}}=\varepsilon_{0} \overline{\mathrm{E}} \nabla \cdot \overline{\mathrm{E}} & \left(\varepsilon_{0} \triangleq \text { Permittivity }\right)
\end{array}
$$

$$
\text { A-1 A-2 A-3 }
$$

$$
\left(\begin{array}{c}
\mathrm{x} \\
\mathrm{y} \\
\mathrm{z}
\end{array}\right) \quad \begin{gathered}
-\frac{1}{c^{2}} \frac{\partial(\overline{\mathrm{E}} \times \overline{\mathrm{H}})}{\partial t}+\varepsilon_{0} \overline{\mathrm{E}}(\nabla \cdot \overline{\mathrm{E}})-\varepsilon_{0} \overline{\mathrm{E}} \times(\nabla \times \overline{\mathrm{E}})+ \\
\mathrm{A}-4 \\
+\mu_{0} \overline{\mathrm{H}}(\nabla \cdot \overline{\mathrm{H}})-\mu_{0} \overline{\mathrm{H}} \times(\nabla \times \overline{\mathrm{H}})=\overline{0}
\end{gathered}
$$


The Electric Radiation Pressure along the $\mathrm{x}$-axis has been compensated by the term Coulomb Interaction Term A-2 in equation (B-9):

The inward oriented Electric Force Density $f_{x i}$ (term A-2 in equation 9$)$ in the $x$ - direction for any arbitrary function $f(x, y, z)$ :

$$
\mathrm{f}_{\mathrm{xi}}=\varepsilon_{0} \mathrm{E}_{x} \frac{\partial \mathrm{E}_{x}}{\partial \mathrm{x}}=\varepsilon_{0} \mathrm{f}(\mathrm{x}, \mathrm{y}) \mathrm{g}\left(\mathrm{t}-\mathrm{z} \sqrt{\varepsilon_{0} \mu_{0}}\right)^{2} \mathrm{f}^{(1,0)}(\mathrm{x}, \mathrm{y})
$$

Compensates the outward oriented Electric Radiation Pressure $f_{x o}$ in the $x$-direction for every arbitrary function $f(x, y, z)$ :

$$
\mathrm{f}_{\mathrm{xo}}=-(\nabla \mathrm{w})_{x}=-\varepsilon_{0} \mathrm{f}(\mathrm{x}, \mathrm{y}) \mathrm{g}\left(\mathrm{t}-\mathrm{z} \sqrt{\varepsilon_{0} \mu_{0}}\right)^{2} \mathrm{f}^{(1,0)}(\mathrm{x}, \mathrm{y})
$$

The Electric Radiation Pressure along the y-axis has been compensated by the Lorentz Interaction Term A-3 in equation (B-9):

The inward oriented Electric Force Density $f_{y i}$ (term A-3 in equation B-9) in the $y$ - direction for every arbitrary function $\mathrm{f}(\mathrm{x}, \mathrm{y}, \mathrm{z})$ :

$$
\mathrm{f}_{\mathrm{yi}}=-\varepsilon_{0}(\overline{\mathrm{E}} \times(\nabla \times \overline{\mathrm{E}}))_{y}=-\varepsilon_{0} \mathrm{f}(\mathrm{x}, \mathrm{y}) \mathrm{g}\left(\mathrm{t}-\mathrm{z} \sqrt{\varepsilon_{0} \mu_{0}}\right)^{2} \mathrm{f}^{(0,1)}(\mathrm{x}, \mathrm{y})
$$

Compensates the outward oriented Electric Radiation Pressure $f_{y o}$ in the y-direction for every arbitrary function $f(x, y, z)$ :

$$
\mathrm{f}_{y o}=-(\nabla \mathrm{w})_{y}=\varepsilon_{0} \mathrm{f}(\mathrm{x}, \mathrm{y}) \mathrm{g}\left(\mathrm{t}-\mathrm{z} \sqrt{\varepsilon_{0} \mu_{0}}\right)^{2} \mathrm{f}^{(0,1)}(\mathrm{x}, \mathrm{y})
$$

This also is valid for a single photon. In the directions perpendicular to the direction of propagation, the electric radiation pressures (B-12) and (B-14) have been compensated completely by the electric interaction forces (B-11) and (B-13). 
The Magnetic Radiation Pressure along the y-axis has been compensated by the term A-4 in equation B-9):

The inward oriented Magnetic Force Density $f_{y i}$ (term A-4 in equation (9)) in the y-direction for every arbitrary function $f(x, y, z)$ : $\mathrm{f}_{\mathrm{yi}}=\mu_{0} \mathrm{H}_{y} \frac{\partial \mathrm{H}_{y}}{\partial \mathrm{y}}=\mu_{0} \mathrm{f}(\mathrm{x}, \mathrm{y}) \mathrm{g}\left(\mathrm{t}-\mathrm{z} \sqrt{\varepsilon_{0} \mu_{0}}\right)^{2} \mathrm{f}^{(0,1)}(\mathrm{x}, \mathrm{y})$

Compensates the outward oriented Magnetic Radiation Pressure $f_{y o}$ in the $\mathrm{y}$-direction for every arbitrary function $\mathrm{f}(\mathrm{x}, \mathrm{y}, \mathrm{z})$ :

$$
\mathrm{f}_{\mathrm{yo}}=-(\nabla \mathrm{w})_{y}=-\mu_{0} \mathrm{f}(\mathrm{x}, \mathrm{y}) \mathrm{g}\left(\mathrm{t}-\mathrm{z} \sqrt{\varepsilon_{0} \mu_{0}}\right)^{2} \mathrm{f}^{(0,1)}(\mathrm{x}, \mathrm{y})
$$

The Magnetic Radiation Pressure along the $\mathrm{x}$-axis has been compensated by the term A-5 in equation (B-9):

The inward oriented Magnetic Force Density $f_{x i}$ (term A-5 in equation 9)) in the $\mathrm{x}$ - direction for every arbitrary function $\mathrm{f}(\mathrm{x}, \mathrm{y}, \mathrm{z})$ : $\mathrm{f}_{\mathrm{xi}}=-\mu_{0}(\overline{\mathrm{H}} \times(\nabla \times \overline{\mathrm{H}}))_{x}=-\mu_{0} \mathrm{f}(\mathrm{x}, \mathrm{y}) \mathrm{g}\left(\mathrm{t}-\mathrm{z} \sqrt{\varepsilon_{0} \mu_{0}}\right)^{2} \mathrm{f}^{(1,0)}(\mathrm{x}, \mathrm{y})$

Compensates the outward oriented Magnetic Radiation Pressure $f_{x o}$ in the $\mathrm{x}$-direction for every arbitrary function $\mathrm{f}(\mathrm{x}, \mathrm{y}, \mathrm{z})$ :

$$
\mathrm{f}_{x o}=-(\nabla \mathrm{w})_{x}=\mu_{0} \mathrm{f}(\mathrm{x}, \mathrm{y}) \mathrm{g}\left(\mathrm{t}-\mathrm{z} \sqrt{\varepsilon_{0} \mu_{0}}\right)^{2} \mathrm{f}^{(1,0)}(\mathrm{x}, \mathrm{y})
$$

This also is valid for a single photon. In the directions perpendicular to the direction of propagation, the magnetic radiation pressures (B-16) and (B-18) have been compensated completely by the magnetic interaction forces (B-15) and (B-17).

For the surface perpendicular to the z-direction of the propagation of a Laser Beam counts that the Electromagnetic Inertia Force compensates exactly the forwardly oriented radiation pressure towards the z-direction.

Exactly at the speed of light, the forwardly oriented Electromagnetic Radiation Pressure along the z-axis has been 
compensated completely by the term backwards oriented Electromagnetic Inertia Term (A-1) in equation (B-9):

The backwards oriented Electromagnetic Inertia Force Density $f_{z b}$ in the z-direction for every arbitrary function $\mathrm{f}(\mathrm{x}, \mathrm{y}, \mathrm{z})$ :

$$
\mathrm{f}_{\mathrm{zb}}=-\frac{1}{c^{2}}\left(\frac{\partial(\overline{\mathrm{E}} \times \overline{\mathrm{H}})}{\partial t}\right)_{z}=-2 \varepsilon_{0} \mathrm{f}(\mathrm{x}, \mathrm{y})^{2} \mathrm{~g}\left(\mathrm{t}-\mathrm{z} \sqrt{\varepsilon_{0} \mu_{0}}\right) \mathrm{g}^{\prime}\left(\mathrm{t}-\mathrm{z} \sqrt{\varepsilon_{0} \mu_{0}}\right)
$$

Compensates exactly at the speed of light:

$$
\mathrm{c}=\frac{1}{\sqrt{\varepsilon_{0} \mu_{0}}}
$$

the forwardly oriented Electric Radiation Pressure $f_{z f}$ in the $y$-direction for every arbitrary function $f(x, y, z)$ :

$$
\mathrm{f}_{\mathrm{zf}}=-(\nabla \mathrm{w})_{\mathrm{z}}=2 \varepsilon_{0} \mathrm{f}(\mathrm{x}, \mathrm{y})^{2} \mathrm{~g}\left(\mathrm{t}-\mathrm{z} \sqrt{\varepsilon_{0} \mu_{0}}\right) \mathrm{g}^{\prime}\left(\mathrm{t}-\mathrm{z} \sqrt{\varepsilon_{0} \mu_{0}}\right)
$$

This also is valid for a single photon. At exactly the "Speed of Light", in the direction of propagation, the electric radiation pressure $(\mathrm{B}-21)$ has been compensated completely by the electromagnetic inertia force density (B-19).

When the beam of light (photon) would propagate in the zdirection slower than the speed of light $\mathrm{c}=1 / \sqrt{\varepsilon_{0} \mu_{0}}$, the radiation pressure would become larger than the inertia force density and the beam of light (photon) would accelerate.

When the beam of light (photon) would propagate in the zdirection faster than the speed of light $\mathrm{c}=1 / \sqrt{\varepsilon_{0} \mu_{0}}$, the radiation pressure would become smaller than the inertia 
force density and the beam of light (photon) would decelerate.

Only at value, when the speed of of light equals exactly $\mathrm{c}=1 / \sqrt{\varepsilon_{0} \mu_{0}}$, then the radiation pressure in the $\mathrm{z}$-direction counterbalances completely the oppositely directed inertia force density of the beam of light (photon).

This is the physical reason why a beam of light propagates exactly with the speed of light. And this is the physical reason why any arbitrary photon always propagates with the universal value for the speed of light in any arbitrary direction. 


\subsection{EM Radiation within a Cartesian Coordinate System in the absence of gravity}

The required Electromagnetic Field Configuration for a perfect Equilibrium in Space and Time follows from the dynamic equilibrium equation (5) and equals in Cartesian Coordinates $\{x, y, z, t\}$ for the Electric Field Components $\mathrm{e}$ $(x, y, z, t)$ :

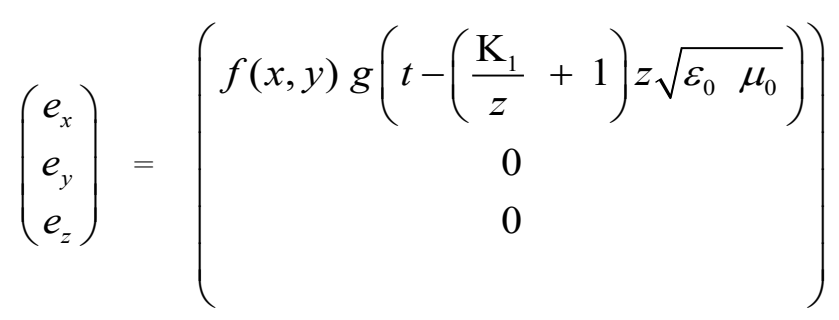

The required Electromagnetic Field Configuration for a perfect Equilibrium in Space and Time follows from the dynamic equilibrium equation (5) and equals in Cartesian Coordinates $\{x, y, z, t\}$ for the Magnetic Field Components $\mathrm{m}$ $(x, y, z, t)$ : 


$$
\left(\begin{array}{l}
m_{x} \\
m_{y} \\
m_{z}
\end{array}\right)=\sqrt{\frac{\varepsilon_{0}}{\mu_{0}}}\left(f(x, y) g\left(t-\left(\frac{\mathrm{K}_{1}}{z}+1\right) z \sqrt{\varepsilon_{0} \mu_{0}}\right)\right)
$$

In which $K_{1}$ is an arbitrary constant. For the divergence-free function $f(x, y)=1$, the solutions (6) and (7) are also the solutions for the known Maxwell Equations. For the nondivergence-free functions $f(x, y)$, the solutions (6) and (7) are not solutions for the Maxwell Equations, which requires divergence-free electromagnetic waves, propagating with the speed of light $c=1 / \sqrt{\varepsilon_{0} \mu_{0}}$, in the absence of any matter.

But they are solutions of the Dynamic Equilibrium Equation (5) and clearly do exist in physics. Comparable with the projection of a slide with a beamer on a flat screen in the $\mathrm{z}$ direction. In which the slide has an arbitrary intensitydivision $f(x, y)$. The information $f(x, y)$ on the slide propagates with the speed of light $c=1 / \sqrt{\varepsilon_{0} \mu_{0}}$ towards the screen in the z-direction in this example. 
3.6 Laser Beam with a Gaussian division in the $x-y$ plane within a Cartesian Coordinate System in the absence of gravity

The required Electromagnetic Field Configuration for a perfect Equilibrium in Space and Time follows from the dynamic equilibrium equation (5).

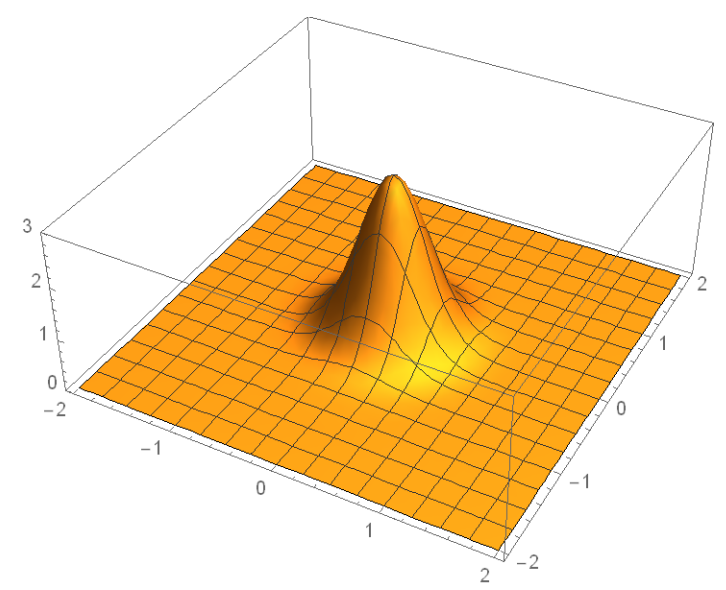

Figure 7 Electromagnetic Field Intensity with a Gaussian division

The required Electromagnetic Field Configuration equals in Cartesian Coordinates $\{x, y, z, t\}$ for the Electric Field Components e $(x, y, z, t)$ : 


$$
\left(\begin{array}{l}
e_{x} \\
e_{y} \\
e_{z}
\end{array}\right)=\left(\begin{array}{cc}
K_{1} \mathrm{e}^{-K_{2}\left(x^{2}+y^{2}\right)} & \operatorname{Sin}\left(\omega\left(t-z \sqrt{\varepsilon_{0}} \mu_{0}\right)\right.
\end{array}\right)
$$

The required Electromagnetic Field Configuration for a perfect Equilibrium in Space and Time follows from the dynamic equilibrium equation (5) and equals in Cartesian Coordinates $\{x, y, z, t\}$ for the Magnetic Field Components $\mathrm{m}$ $(x, y, z, t):$
$\left(\begin{array}{l}m_{x} \\ m_{y} \\ m_{z}\end{array}\right)=\sqrt{\frac{\varepsilon_{0}}{\mu_{0}}}\left(\begin{array}{c}0 \\ K_{1} \mathrm{e}^{-K_{2}\left(x^{2}+y^{2}\right)} \operatorname{Sin}\left(\omega\left(t-z \sqrt{\varepsilon_{0} \mu_{0}}\right)\right) \\ 0\end{array}\right)$

In which $K_{1}$ is an arbitrary constant. The Gaussian division is not a solution of the Maxwell Equations, because the divergence of the Electric field intensity does not equal zero

$$
\nabla \cdot \overline{\mathrm{E}}=-2 \mathrm{e}^{-\mathrm{K}_{2}\left(\mathrm{x}^{2}+\mathrm{y}^{2}\right)} \times \mathrm{K}_{1} \mathrm{~K}_{2} \operatorname{Sin}\left(\left(t-z \sqrt{\varepsilon_{0} \mu_{0}}\right) \omega\right)
$$

And the divergence of the Magnetic field intensity does not equal zero. 


$$
\nabla \cdot \overline{\mathrm{H}}=-\sqrt{\frac{\varepsilon_{0}}{\mu_{0}}}\left(2 \mathrm{e}^{-\mathrm{K}_{2}\left(\mathrm{x}^{2}+\mathrm{y}^{2}\right)} \mathrm{y} \mathrm{K}_{1} \mathrm{~K}_{2} \operatorname{Sin}\left(\left(t-z \sqrt{\varepsilon_{0} \mu_{0}}\right) \omega\right)\right)
$$

For the non-divergence-free functions

$$
f(x, y)=K_{1} \mathrm{e}^{-K_{2}\left(x^{2}+y^{2}\right)} \operatorname{Sin}\left(\omega\left(t-z \sqrt{\varepsilon_{0} \mu_{0}}\right)\right) \text {, the }
$$

solutions (6) and (7) are not solutions for the Maxwell Equations, which requires divergence-free electromagnetic waves, propagating with the speed of light $c=1 / \sqrt{\varepsilon_{0} \mu_{0}}$, in the absence of any matter. But they are solutions of the Dynamic Equilibrium Equation (5) and clearly do exist in physics. Comparable with the projection of a slide with a beamer on a flat screen in the z-direction. In which the slide has an arbitrary intensity-division $f(x, y)$ and the intensity is not Divergence Free. The information $f(x, y)$ on the slide propagates with the speed of light $c=1 / \sqrt{\varepsilon_{0} \mu_{0}}$ towards the screen in the z-direction in this example and is a real physical possibility and is not a solution of the Maxwell equations but is a fundamental solution of the Dynamic Equilibrium Equation (5) and clearly does exist in physics. 


\subsection{EM Radiation within a Cartesian Coordinate System under the influence of a Longitudinal Gravitational}

Field g

The required Electromagnetic Field Configuration for a perfect Equilibrium in Space and Time for a Longitudinal Gravitational Field (The Light propagates in the same zdirection as the z-direction of the Gravitational Field ) follows from the Dynamic Equilibrium Equation (5) and equals in Cartesian Coordinates $\{x, y, z, t\}$ for a gravitational field "g" for the Electric Field Components e $(x, y, z, t)$ :

$\left(\begin{array}{l}e_{x} \\ e_{y} \\ e_{z}\end{array}\right)=\left(\begin{array}{cc}\mathrm{e}^{-\frac{1}{2} \mathrm{~g} \varepsilon_{0} \mu_{0} \mathrm{z}} & f(x, y) g\left(t-z \sqrt{\varepsilon_{0} \mu_{0}}\right) \\ 0 \\ 0\end{array}\right)$

The required Electromagnetic Field Configuration for a perfect Equilibrium in Space and Time for a Longitudinal Gravitational Field (The Light propagates in the same zdirection as the z-direction of the Gravitational Field ) follows from the Dynamic Equilibrium Equation (5-a) and equals in Cartesian Coordinates $\{x, y, z, t\}$ for a gravitational field " $\mathrm{g}$ " for the Magnetic Field Components $\mathrm{m}(x, y, z, t)$ : 


$$
\left(\begin{array}{l}
m_{x} \\
m_{y} \\
m_{z}
\end{array}\right)=\sqrt{\frac{\varepsilon_{0}}{\mu_{0}}}\left(\begin{array}{c}
0 \\
\mathrm{e}^{-\frac{1}{2} \mathrm{~g} \varepsilon_{0} \mu_{0} \mathrm{z}}
\end{array}\right.
$$

Equation (6-a) and (7-a) are solutions of (5-a) under the influence of a Longitudinal Gravitational field with field intensity "g" that acts along the z-direction while the electromagnetic wave is also propagating in the $\mathrm{z}$-direction.

The electromagnetic wave is propagating with the unaltered speed of light $c=1 / \sqrt{\varepsilon_{0} \mu_{0}}$, independently of the strength $g$ of the gravitational field in the z-direction. However, the amplitude of the electromagnetic wave becomes dependently of the gravitational intensity " $\mathrm{g}$ " and the distance " $\mathrm{z}$ " and changes along the $\mathrm{z}$-axis with the electromagneticgravitational interaction term $\mathrm{e}^{-\frac{1}{2} \mathrm{~g} \varepsilon_{0} \mu_{0} \mathrm{z}}$.

In this example is chosen for e.g. a laser beam positioned vertically on the ground on earth, shining vertically against the gravitational field " $\mathrm{g}$ " of the earth. Because the laser beam presents electromagnetic energy, the beam has electromagnetic mass. The potential energy of the electromagnetic mass is increasing while the laser light is propagating upwards, against the direction of the gravitational field. Because of the law of conservation of Energy, the electromagnetic energy is decreasing over a distance " $\mathrm{z}$ " proportional with the same amount $\mathrm{e}^{-\mathrm{g} \varepsilon_{0} \mu_{0} \mathrm{z}}$ 
as the potential energy of the electromagnetic mass is increasing. 


\subsection{The Real Light Intensity of the Sun, measured in our Solar System, including Electromagnetic Gravitational Conversion (EMGC)}

When a beam of light leaves the surface of the sun, the light will travel in the radial direction of the radial gravitational field caused by the sun. The required Electromagnetic Field Configuration for a perfect Equilibrium in Space and Time for a Radial Gravitational Field (The Light propagates in the same radial-direction as the radial-direction of the Gravitational Field ) follows from Dynamic Equilibrium Equation (5) and equals in Spherical Coordinates $\{r, \theta, \varphi, t\}$ for a gravitational field " $\mathrm{g}(\mathrm{r})$ " for the Electric Field Components $\mathrm{e}(r, \theta, \varphi, t)$ :

$$
\left(\begin{array}{l}
e_{r} \\
e_{\theta} \\
e_{\varphi}
\end{array}\right)=\left(\begin{array}{ccc}
\frac{1}{r} & \mathrm{e}^{\frac{\mathrm{Gm}_{1} \varepsilon_{0} \mu_{0}}{2 \mathrm{r}}} & \mathrm{K} 1 \mathrm{~g}\left(\mathrm{t}-\mathrm{r} \sqrt{\varepsilon_{0} \mu_{0}}\right) \\
& & 0 \\
& & 0
\end{array}\right)
$$

The required Electromagnetic Field Configuration for a perfect Equilibrium in Space and Time for a Radial Gravitational Field (The Light propagates in the same radialdirection as the radial-direction of the Gravitational Field ) follows from the Dynamic Equilibrium Equation (5) and equals in Spherical Coordinates $\{r, \theta, \varphi, t\}$ for a gravitational field " $\mathrm{g}(\mathrm{r})$ " for the Magnetic Field Components $\mathrm{m}(r, \theta, \varphi, t)$ : 


$$
\left(\begin{array}{l}
m_{x} \\
m_{y} \\
m_{z}
\end{array}\right)=\sqrt{\frac{\varepsilon_{0}}{\mu_{0}}}\left(\begin{array}{cc}
\frac{1}{r} & \mathrm{e}^{\frac{\mathrm{G} \mathrm{m}_{1} \varepsilon_{0} \mu_{0}}{2 \mathrm{r}}} \\
\mathrm{K} 1 \mathrm{~g}\left(\mathrm{t}-\mathrm{r} \sqrt{\varepsilon_{0} \mu_{0}}\right) \\
0
\end{array}\right)(7-\mathrm{b})
$$

Equation (6-b) and (7-b) are solutions of (5-a) under the influence of a Radial Gravitational field with field a gravitational field intensity " $\mathrm{g}(\mathrm{r})$ " that acts along the radialdirection while the electromagnetic wave is also propagating in the radial-direction.

When a light beam leaves the surface of the sun, the intensity will decrease according (6-b). At earth, the measured intensity will be according (5-a) and (6-b):

$$
I=\frac{I_{0} e^{\frac{\mathrm{G} \mathrm{m}_{1} \varepsilon_{0} \mu_{0}}{\mathrm{r}}}}{4 \pi \mathrm{r}^{2}}
$$

A beam of light represents an amount of electromagnetic energy. Which equals an amount of electromagnetic mass. This amount of electromagnetic mass is moving with the speed of light in the opposite direction of a (radial) gravitational field and gains potential energy. Because the law of conservation of energy, a part of the electromagnetic energy of the light beam has to be converted into potential energy according equation (6-c). 


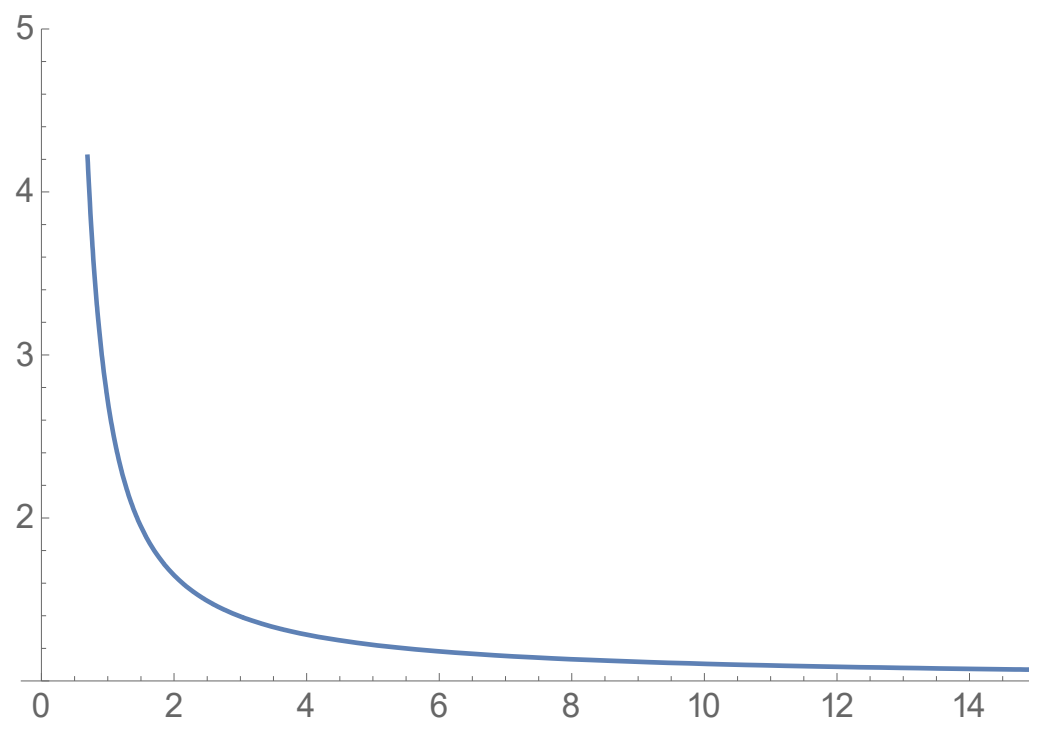

Figure 8 Electromagnetic Gravitational Conversion

Term $e^{\frac{G m_{1} \varepsilon_{0} \mu_{0}}{r}}$

For a radius of the sun equals $695,508[\mathrm{~km}]$ and a distance from the sun to the earth of $149,600,000$ [km], the Electromagnetic Gravitational Conversion (EMGC) term equals:

$$
C_{E M G C}=\frac{\mathrm{e}^{\frac{\mathrm{Gm}_{1} \varepsilon_{0} \mu_{0}}{\mathrm{r}_{1}}}}{\mathrm{e}^{\frac{\mathrm{Gm} \varepsilon_{1} \varepsilon_{0}}{\mathrm{r} 2}}}=\frac{\mathrm{e}^{\frac{1}{\mathrm{r}_{1}}}}{\mathrm{e}^{\frac{1}{\mathrm{r} 2}}}=4.1877534
$$

This means that the real intensity of the light at the surface of the sun is about 4 times higher than the intensity which would have been calculated in a classical way from the 
sunlight intensity measured on earth, due to Electromagnetic Gravitational Conversion.

Equations (6-b) and (7-b) are solutions of (5-a) under the influence of a Radial Gravitational field with field intensity " $g(r)$ " that acts along the radial-direction while the electromagnetic wave is also propagating in the same radialdirection. The electromagnetic wave is propagating with the unaltered speed of light $c=1 / \sqrt{\varepsilon_{0} \mu_{0}}$, independently of the strength $g(r)$ of the gravitational field in the radial-direction. However, the amplitude of the electromagnetic wave becomes dependently of the gravitational intensity " $\mathrm{g}(\mathrm{r})$ " and the distance " $r$ " and changes along the radial direction due to the Electromagnetic-Gravitational Conversion term $C_{E M G C}=\mathrm{e}^{\frac{\mathrm{Gm}_{1} \varepsilon_{0} \mu_{0}}{\mathrm{r}}}$.

Because of the law of conservation of Energy, the electromagnetic energy of the light emitted by the sun is decreasing over a distance " $r$ " proportional with the same amount $E m G C=e^{\frac{\mathrm{Gm}_{1} \varepsilon_{0} \mu_{0}}{\mathrm{r}}}$ as the potential energy of the electromagnetic mass of the light emitted by the sun is increasing. 


\subsection{The Boundaries of our Universe}

When a mass, for example a ball, has been thrown into the air, the mass moves against the direction of the gravitational field of the earth and kinetic energy has been converted into potential energy. When the ball has reached the maximum height, the ball falls back towards the earth and the potential energy has been converted again into kinetic energy. The total sum of kinetic energy and potential energy remains constant.

The Cosmic Microwave Background Radiation (CMBR ) is light (electromagnetic radiation) and represents an amount of electromagnetic energy. Which equals an amount of electromagnetic mass. This amount of electromagnetic mass is moving with the speed of light in the opposite direction of a (radial) gravitational field and gains potential energy. Because the law of conservation of energy, a part of the electromagnetic energy of the light beam has to be converted into potential energy according equation (6-c).

In a comparable way the Cosmic Microwave Background Radiation (CMBR ) (light, electromagnetic) radiation moves away from the center of our Universe, gaining Potential Energy by moving against the (Radial) Gravitational field of our Universe. Until the Intensity of the light vanishes into zero at the boundaries of our Universe and has the potency to fall back towards its origin gaining an non-imaginable amount of light intensity by the conversion of potential energy into electromagnetic energy. 
In this model of the Universe, the electromagnetic radiation within the universe, is locked up in its own gravitational field. And the whole Universe can be considered to be a gigantic Black Hole with a diameter over 5 billion lightyears.

Depending on the the electromagnetic stability at the boundaries of our Universe a periodic model of our Universe is possible like the ball being thrown into the air and falling back towards the location where it came from, or the universe finally disappearing into the infinite space.

These possibilities depend on the radial dependent electromagnetic intensity function of the Cosmic Microwave Background Radiation (CMBR) Table 1. 


\subsection{The Origin of Dark Matter}

The Cosmic Microwave Background Radiation (CMBR) is the dominant radiation field in the Universe, and one of the most powerful cosmological tools that has yet been found, 25 years after its discovery by Penzias \& Wilson (1965) .

Within a few years of the discovery of the CMBR, it was established the radiation field is close to isotropic, with a spectrum characterized by a single temperature, $T_{\text {rad }} \approx 2.7 \mathrm{~K}$. The specific intensity of the radiation is therefore close to:

$$
I_{f}=\frac{2 \mathrm{~h} \mathrm{f}^{3}}{c^{2}}\left(\mathrm{e}^{h f / k_{B} T_{\text {rad }}}-1\right)^{-1}
$$

which corresponds to a peak brightness $I_{\max } \sim 3.7 \times 10^{-18} \mathrm{~W}$ $\mathrm{m}^{-2} \mathrm{~Hz}^{-1} \mathrm{sr}^{-1}$ at $\mathrm{f}_{\max } \sim 160 \mathrm{GHz}$ and an energy density $i_{f} \sim 4 \mathrm{x}$ $10^{-14} \mathrm{~J} \mathrm{~m}^{-3}$, which can also be expressed as a mass density Qem $\sim 5 \times 10^{-31} \mathrm{~kg} \mathrm{~m}^{-3}$.

When from the earth an Electromagnetic Mass Density has been measured which equals $\rho_{\mathrm{em}}=5 \times 10^{-36} \mathrm{~kg} / \mathrm{m}^{3}$, the Total Mass of the Universe can be roughly calculated without and with regarding the effect of Electromagnetic Gravitational Conversion.

At the Origin of the Universe, at the start of the Big Bang, a large amount of electromagnetic radiation has been blown into the universe, which has been measured on earth as the well-known CMBR. The radiation, traveling for billions of 181 
years against the gravitational field of the origin of the universe and has gained during that time an enormous amount of potential energy. Because of the conversation of energy and the transfer of electromagnetic energy into potential energy, the intensity of the CMBR, measured on earth, has been much lower than the real intensity has been at the origin.

As an example a visible universe like a sphere has been chosen with a radius of $4.410^{26}[\mathrm{~m}]$ and the earth located in the middle between the origin of the Big Bang and the outer boundaries of the visible Universe. As an example, two basic calculations have been done. The first one, calculating the total electromagnetic mass in the universe without taking into account the Electromagnetic-Gravitational Interaction.

$$
\begin{aligned}
& M_{\text {UNIVERSE }}=\int_{R_{1}}^{R_{2}} \rho_{E M} 4 \pi \mathrm{r}^{2} \mathrm{dr}=\int_{0.0113}^{4.4 \times 10^{26}} \frac{5 \times 10^{-36}}{\left(r / 2.2 \times 10^{26}\right)^{2}} 4 \pi \mathrm{r}^{2} \mathrm{dr}= \\
& =1.33810^{45}[\mathrm{~kg}]
\end{aligned}
$$

The second calculation has been done by taking into account the Electromagnetic-Gravitational Interaction, including the Electromagnetic-Gravitational Conversion term

$$
\begin{aligned}
& C_{E M G C}=\mathrm{e}^{\frac{\mathrm{G} \mathrm{m}_{1} \varepsilon_{0} \mu_{0}}{\mathrm{r}}} \\
& M_{\text {UNVERSE }}=\int_{R_{1}}^{R_{2}} \rho_{E M} \mathrm{e}^{\frac{1}{r}} 4 \pi \mathrm{r}^{2} \mathrm{dr}=\int_{0.0113}^{4.4 \times 10^{26}} \frac{5 \times 10^{-36}}{\left(r / 2.2 \times 10^{26}\right)^{2}} \frac{e^{\frac{1}{r}}}{e^{\frac{1}{2.2 \times 10^{26}}}} 4 \pi \mathrm{r}^{2} \mathrm{dr}= \\
& =1.077 \times 10^{53}[\mathrm{~kg}]
\end{aligned}
$$


Including the effect of Electromagnetic-Gravitational Conversion changes an almost negligible effect of the CMBR on the total mass of the Universe into an effect that can easily explain the total mass of the Universe while neglecting the influences of the mass of the galaxies. And makes the Theory of Dark Matter avoidable. 


\subsection{Electromagnetic Radiation within a Spherical} Coordinate System

The Spherical Coordinate System $\{r, \theta, \varphi, t\}$ is parameterized by the radius $r$ of the Sphere, the polar angle $\theta$ and the azimuthal angle $\varphi$ and the time t.

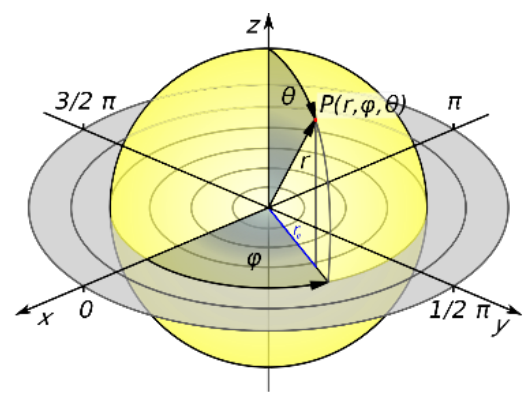

Figure 9 Spherical Coordinate System

The required Electromagnetic Field Configuration for a perfect Equilibrium in Space and Time follows from equation (5) and equals in Spherical Coordinates $\{r, \theta, \varphi, t\}$ for the Electric Field Components e $(\theta, r, \varphi, t)$ :

$$
\left(\begin{array}{l}
e_{r} \\
e_{\theta} \\
e_{\varphi}
\end{array}\right)=\left(\begin{array}{c}
0 \\
\frac{1}{r} f(\theta, \varphi) g\left(t-\left(\frac{\mathrm{K}_{1}}{r}+1\right) r \sqrt{\varepsilon_{0} \mu_{0}}\right) \\
0
\end{array}\right)
$$

The required Electromagnetic Field Configuration for a perfect Equilibrium in Space and Time follows from equation 
(4) and equals in Spherical Coordinates $\{r, \theta, \varphi, t\}$ for the Magnetic Field Components $\mathrm{m}(\theta, r, \varphi, t)$ :

$$
\left(\begin{array}{l}
m_{r} \\
m_{\theta} \\
m_{\varphi}
\end{array}\right)=\sqrt{\frac{\varepsilon_{0}}{\mu_{o}}}\left(\begin{array}{c}
0 \\
0 \\
\frac{1}{r} f(\theta, \varphi) g\left(t-\left(\frac{\mathrm{K}_{1}}{r}+1\right) r \sqrt{\varepsilon_{0} \mu_{0}}\right)
\end{array}\right)
$$

For the divergence-free function $f(\theta, \varphi)=1$, the solutions (8) and (9) are also the solutions for the known Maxwell Equations. For the non-divergence-free functions $f(\theta, \varphi)$, the solutions (8) and (9) are no solutions for the Maxwell Equations, which require divergence-free electromagnetic waves in the absence of any matter. ${ }^{(29,35,36,37,38)}$. They are however solutions of the DEE (5) and clearly they do exist in physics. Like the radiation of an inhomogeneous point light source like a LED. 


\subsection{Confined Electromagnetic Radiation within a Spherical Coordinate System through Electromagnetic- Gravitational Interaction}

In physics it has been in generally assumed that the speed of light $c=1 / \sqrt{\varepsilon_{0} \mu_{0}}$ is a physical constant. In this paragraph the possibilities will be discussed of a variable speed of light $(10,11,12)$. that can vary from zero until values higher than c. The only requirement for the existence of an Electromagnetic Field Configuration will be the requirement of a perfect equilibrium in space-time for the chosen electromagnetic field configuration $^{(13,14,15)}$. This single unique requirement will always be a solution of the DEE (5).

The required Electromagnetic Field Configuration for a perfect Equilibrium in Space and Time ${ }^{(16,17,18,30,31,39}$ in respectively the : $\theta$-direction $\left(f_{\theta}=0\right)$ and the $\varphi$-direction : $(f \varphi=0)$ follows from equation (5).

In Spherical Coordinates $\{r, \theta, \varphi, t\}$ the solution for the DEE (5) for the Electric Field Components e $(\theta, r, \varphi, t)$ equals:

$$
\left(\begin{array}{l}
e_{r} \\
e_{\theta} \\
e_{\varphi}
\end{array}\right)=\left(\begin{array}{c}
0 \\
f(r) g(\theta) h(\varphi) \operatorname{Sin}(\omega t) \\
-f(r) g(\theta) h(\varphi) \operatorname{Cos}(\omega t)
\end{array}\right)
$$

In Spherical Coordinates $\{r, \theta, \varphi, t\}$ the solution for the DEE

(5) for the Magnetic Field Components $m(\theta, r, \varphi, t)$ in 
respectively the : $\theta$-direction $\left(f_{\theta}=0\right)$ and the $\varphi$-direction: $(f \varphi=0)$ for the magnetic field components follows from equation $(5)^{(29,30,31)}$. and equals:

$$
\left(\begin{array}{l}
m_{r} \\
m_{\theta} \\
m_{\varphi}
\end{array}\right)=\left(\begin{array}{c}
0 \\
f(r) g(\theta) h(\varphi) \operatorname{Cos}(\omega t) \\
f(r) g(\theta) h(\varphi) \operatorname{Sin}(\omega t)
\end{array}\right)
$$

Equation (4) gives the 3-dimensional force density $f^{a}$ of an Electro-Magnetic Field Configuration ${ }^{(19,20,21)}$. in a coordinate free vector equation.

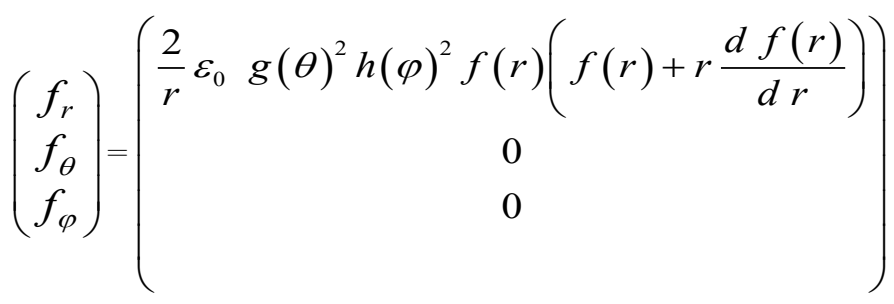

It follows from equation (4) that the radiation pressure in radial direction does not counterbalance and does not equal zero.

The energy-density $\mathrm{w}_{\mathrm{m}}$ of the Electromagnetic Configuration is essential for the calculation of the inward bounded gravitational pressure. The electromagnetic field configuration (10) and (11) for the functions 
$\mathrm{g}(\theta)=1$ and $\mathrm{h}(\varphi)=1$, results into the electromagnetic energy-density $\mathrm{w}_{\mathrm{em}}$ :

$$
\begin{aligned}
& \left(\begin{array}{l}
e_{r} \\
e_{\theta} \\
e_{\varphi}
\end{array}\right)=\left(\begin{array}{c}
0 \\
\mathrm{f}(\mathrm{r}) \operatorname{Sin}(\omega \mathrm{t}) \\
-\mathrm{f}(\mathrm{r}) \operatorname{Cos}(\omega \mathrm{t})
\end{array}\right) \quad\left(\begin{array}{l}
m_{r} \\
m_{\theta} \\
m_{\varphi}
\end{array}\right)=\left(\begin{array}{c}
0 \\
\mathrm{f}(\mathrm{r}) \operatorname{Cos}(\omega \mathrm{t}) \\
\mathrm{f}(\mathrm{r}) \operatorname{Sin}(\omega \mathrm{t})
\end{array}\right) \\
& \mathrm{w}_{\mathrm{em}}=\left(\frac{\mu_{0}}{2}(\overline{\mathrm{m}} \cdot \overline{\mathrm{m}})+\frac{\varepsilon_{0}}{2}(\overline{\mathrm{e}} \cdot \overline{\mathrm{e}})\right)=\varepsilon_{0} \mathrm{f}(r)^{2}
\end{aligned}
$$

According to Einstein's mass-energy equivalent $\mathrm{W}=\mathrm{m} \mathrm{c}^{2}$ ,the specific electromagnetic mass ${ }^{(22,23,24,29)}$. density $\rho_{\mathrm{em}}$ equals:

$$
\rho_{\mathrm{em}}=\frac{1}{\mathrm{c}^{2}}\left(\frac{\mu_{0}}{2}(\overline{\mathrm{m}} \cdot \overline{\mathrm{m}})+\frac{\varepsilon_{0}}{2}(\overline{\mathrm{e}} \cdot \overline{\mathrm{e}})\right)=\varepsilon_{0}^{2} \mu_{0} \mathrm{f}(\mathrm{r})^{2}
$$

The total electromagnetic mass ${ }^{(25,6,27,29,38)} . \mathrm{M}_{\mathrm{em}}$ within a sphere with radius $\mathrm{R}$ equals:

$$
\mathrm{M}_{e m}=4 \pi \varepsilon_{0}^{2} \mu_{0} \int_{0}^{\mathrm{R}} \mathrm{r}^{2} \mathrm{f}(\mathrm{r})^{2} \mathrm{dr}
$$

At a distance $r$ from the center of the sphere, the total electromagnetic mass $\mathrm{M}_{\mathrm{em}}$ within the sphere ${ }^{(25,26,27,30)}$. causes, according Newton's Shell Theorem ${ }^{(28,29,30)}$, a gravitational field strength $g_{e m}$ : 


$$
\mathrm{g}_{\mathrm{em}}=\frac{4}{\mathrm{r}^{2}} \pi \varepsilon_{0}^{2} \mu_{o} \mathrm{G}_{1} \int_{0}^{\mathrm{R}} \mathrm{r}^{2} \mathrm{f}(\mathrm{r})^{2} \mathrm{dr}
$$

In which $\mathrm{G}_{1}$ is the gravitational constant and equals $\mathrm{G}_{1}=6.6740810^{-11}\left[\mathrm{~m}^{3} \mathrm{~kg}^{-1} \mathrm{~s}^{-2}\right]$. The gravitational inwards bounded radiation pressure ${ }^{(29,31,32,33)}$. follows from (16):

$$
\mathrm{f}_{G R A V}=\rho_{\mathrm{em}} \mathrm{g}_{\mathrm{em}}=\frac{4}{\mathrm{r}^{2}} \pi \varepsilon_{0}^{4} \mu_{0}^{2} \mathrm{G}_{1} \mathrm{f}(r)^{2} \int_{0}^{\mathrm{R}} \mathrm{r}^{2} \mathrm{f}(\mathrm{r})^{2} \mathrm{dr}
$$

When there is a perfect equilibrium between the outwards bounded electromagnetic radiation pressure $f_{R A D}$ and the inward bounded gravitational pressure ${ }^{(29,34,35,38,39)}$. $f_{\text {GRAV we }}$ find from (12) and (17) the radius of the boundary sphere of the enclosed radiation $R_{B O U N D A R Y}$. for the functions

$$
\begin{aligned}
& \mathrm{g}(\theta)=1 \text { and } \mathrm{h}(\varphi)=1 \\
& \mathrm{f}_{\mathrm{RAD}}=\frac{2}{\mathrm{r}} \varepsilon_{0} \mathrm{f}(\mathrm{r})\left(\mathrm{f}(\mathrm{r})+\mathrm{r} \frac{\mathrm{d} \mathrm{f}(\mathrm{r})}{\mathrm{dr}}\right)= \\
& =\frac{4}{\mathrm{r}^{2}} \pi \varepsilon_{0}^{4} \mu_{0}^{2} \mathrm{G}_{1} \mathrm{f}(\mathrm{r})^{2} \int^{R-B O U M N D A R Y} \mathrm{r}^{2} \mathrm{f}(\mathrm{r})^{2} \mathrm{dr}=\mathrm{f}_{\text {GRAV }}
\end{aligned}
$$

In the following example we choose for the function $\mathrm{f}(\mathrm{r})$

$$
\mathbf{f}(\mathbf{r})=\mathrm{K}_{1} \mathbf{r}^{\mathrm{n}}
$$


Substituting (19) in (18) results in the equation for $R_{\text {BONNDARY }}$ :

$$
\frac{8.019056 \times 10^{-94} \mathrm{~K}^{6} \mathrm{R}_{\text {BOUNDARY }}^{1+6 n}}{3+2 \mathrm{n}}=1.7708376 \times 10^{-11} \mathrm{~K}^{2}(1+\mathrm{n}) \mathrm{R}_{\text {BOUNDARY }}^{-1+2 n}
$$


Table 1:

Values Values for R $\mathrm{R}_{\text {BOUNDARY }}$ Frequenc Area for $n$ $\mathrm{K}_{1}$ [m] y $\omega_{0}$

$-10$ $0.000724222 .6 \times$ $7 \quad 10^{12}$

$-4 \quad 1$

$5.59402393 .37 \times$ $\times 10^{-10} \quad 10^{18}$

$-2 \quad 1$

$6.7798267 \quad 2.78 \times$ $\times 10^{-28} \quad 10^{36}$ Electromag $1.35596531 .39 \times$ netic

$-2 \quad 2$

$\times 10^{-27}$ $10^{36}$ Particle

$-2 \quad 10^{18}$

$6.7798267 \quad 2.78 \times$ $\times 10^{-10}$ $10^{18}$ $3.06229216 .15 \times$

$-1.6 \quad 1$

$\times 10^{-45}$ $10^{53}$

$4.7888625 \quad 3.94 \times$

$-1.51 \quad 1$

$\times 10^{-52}$ $10^{60}$

$9.4264902 \quad 2.0 \times$

$-1.51 \quad 10^{18}$

$\times 10^{-17}$ $10^{25}$

Infinite

Solution of

$-1 \quad 1$

$1.2126075 \quad 1.55 \times$

$\times 10^{267} \quad 10^{-258}$

$-0.5 \quad 1$

$2.1755186 \quad 8.66 \times$

$\times 10^{54}$ $10^{-46}$

$-0.5 \quad 10^{18}$

$-0.5 \quad 10^{36}$ (Electro$\underline{\text { magneticall }}$ y

$\underline{\text { Controlled) }}$

$-0.9 \quad 1$$$
10
$$$$
\times 10^{54}
$$

Electromag netic Black Hole

(Gravitation ally

Controlled) 


\subsection{The fundamental conflict between Causality and Probability}

The beginning of the conflict between Causality and Probability in Physics started at the historic invitation-only "Conseil Solvay" in 1911. Since that conference Albert Einstein has always defended the fundamental concept of Causality and the logical grounds for Causality and Effect while Niels Bohr has always defended the fundamental concept of Probability in which there is no relationship between Causality and Effect. The most fundamental and famous conference was the October 1927 "Fifth Solvay International Conference on Electrons and Photons" where the newly formulated Quantum Theory, based on Probability, had been accepted. Since then Quantum Physics has grown in power and has always been grounded on the material waves, for the first time mathematically described by Erwin Schrödinger and designated to be probability waves.

In Ref. (29) page 206 the "law of continuity" for electromagnetic radiation has been presented in equation (42), By presenting the electromagnetic field in a complex configuration in equation (48) on page 207, the electromagnetic continuity equation (42) has been presented as the quantum mechanical Schrödinger wave equation (53) on page 207. However the function $\psi$ in (53) does not represent the quantum mechanical probability function but represents the confined electromagnetic field with harmonic frequency $\omega_{0}$ in which the magnetic part has been presented as the real function $\bar{B} / \mu$ and the electric part has been presented as the imaginary function $i \overline{\mathrm{E}} / \mathrm{c}$. With "i" the 192 
imaginary number $\sqrt{-1}$. However as well as the electric part as the magnetic part of the confined electromagnetic field are both real.

The quantum mechanical wave function $\psi$ is in real a vector function $\bar{\phi}$ which equals:

$$
\bar{\phi}=\frac{1}{\sqrt{2 \mu}}\left(\overline{\mathrm{B}}+\mathrm{i} \frac{\overline{\mathrm{E}}}{\mathrm{c}}\right)
$$

and the complex conjugated vector function equals:

$$
\overline{\phi^{*}}=\frac{1}{\sqrt{2 \mu}}\left(\overline{\mathrm{B}}-\mathrm{i} \frac{\overline{\mathrm{E}}}{\mathrm{c}}\right)
$$

And the dot product equals the electromagnetic energy density w:

$\bar{\phi} \cdot \overline{\phi^{*}}=\frac{1}{2 \mu}\left(\overline{\mathrm{B}}+\mathrm{i} \frac{\overline{\mathrm{E}}}{c}\right) \cdot\left(\overline{\mathrm{B}}-\mathrm{i} \frac{\overline{\mathrm{E}}}{c}\right)=\frac{1}{2} \mu \mathrm{H}^{2}+\frac{1}{2} \varepsilon \mathrm{E}^{2}=\mathrm{w}$

The cross product is proportional to the Poynting vector (ref. 29, page 202, equation 15 ).

$\bar{\phi} \times \overline{\phi^{*}}=\frac{1}{2 \mu}\left(\overline{\mathrm{B}}+\mathrm{i} \frac{\overline{\mathrm{E}}}{c}\right) \times\left(\overline{\mathrm{B}}-\mathrm{i} \frac{\overline{\mathrm{E}}}{c}\right)=\mathrm{i} \sqrt{\varepsilon \mu} \overline{\mathrm{E}} \times \overline{\mathrm{H}}=\mathrm{i} \sqrt{\varepsilon \mu} \overline{\mathrm{S}}$

In Ref. (29) on page 208 the "law of continuity" for electromagnetic radiation has been presented in equation (57). The vector function $\bar{\phi}$ represents the confined electromagnetic field with harmonic frequency $\omega_{0}$ in which the magnetic part has been presented as the real vector function $\bar{B}$ and the electric part has been presented as the 193 
imaginary vector function $i \overline{\mathrm{E}} / \mathrm{c}$. By substituting the complex vector function $\bar{\phi}$ in (57) and using the relativistic Lorentz transformations, the quantum mechanical relativistic Dirac equation has been derived in equation (102) on page 213. This has been published in 1995 in Physics Essays in $\underline{\mathrm{A}}$ Continuous model of Matter (DOI: 10.13140/RG.2.2.25149.77281). (ref. 29).

This fundamental conflict is still going on and in this article mathematical results are presented in Table 1 in which the famous De Broglie waves, the material waves, designated as probability waves are electromagnetic waves with one harmonic frequency $\omega$, confined by electromagneticgravitational interaction. Electromagnetic waves fully grounded on Causality and Effect.

Table 1 presents several values for the calculated Equilibrium Radius $R_{B O U N D A R Y}(20)$ at different values $\mathrm{K}_{1}$ and $\mathrm{n}$ in Equation (18), (19) and (20) for the harmonic Electromagnetic-Gravitationally confined Electromagnetic waves with frequency $\omega_{0}$ : 


\subsection{Confined Electromagnetic Radiation within a Toroidal Coordinate System}

The Toroidal Coordinate System $\{\theta, r, \varphi, R, t\}$ is parameterized by the large radius $\mathrm{R}$ of the Torus. The Toroidal Coordinate System is obtained by rotating bipolar coordinates $\{r, \varphi\}$ around an axis perpendicular to the axis connecting the two foci. The coordinate $\{\theta\}$ specifies the angle of rotation.

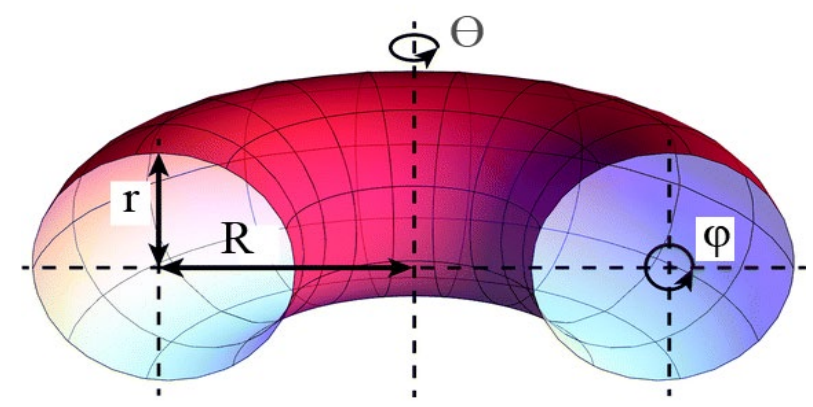

Figure 10 Toroidal Coordinate System 
The required Electromagnetic Field Configuration for a perfect Equilibrium in Space and Time equals in Toroidal Coordinates $\{\theta, r, \varphi, t\}$ for the Electric Field Components $\mathrm{e}$ $(\theta, r, \varphi, t)$ :

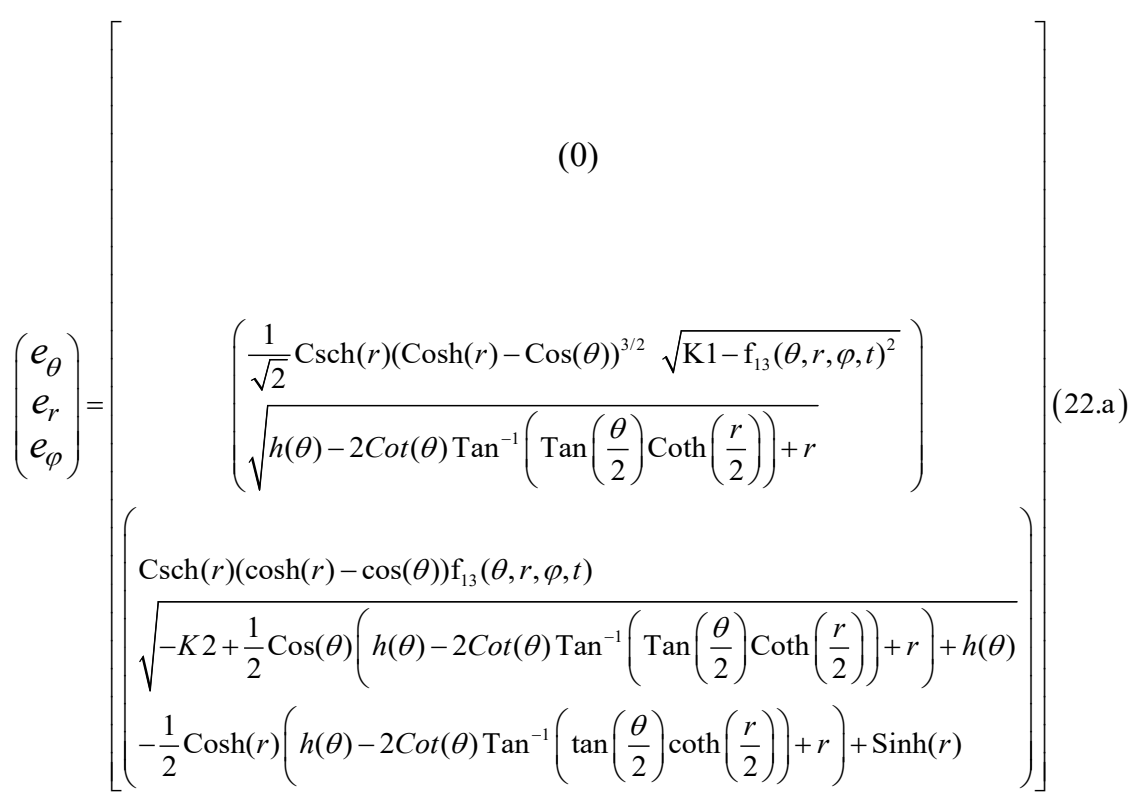


The required Electromagnetic Field Configuration for a perfect Equilibrium in Space and Time equals in Toroidal Coordinates $\{\theta, r, \varphi, t\}$ for the Magnetic Field Components $\mathrm{m}$ $(\theta, r, \varphi, t)$ :

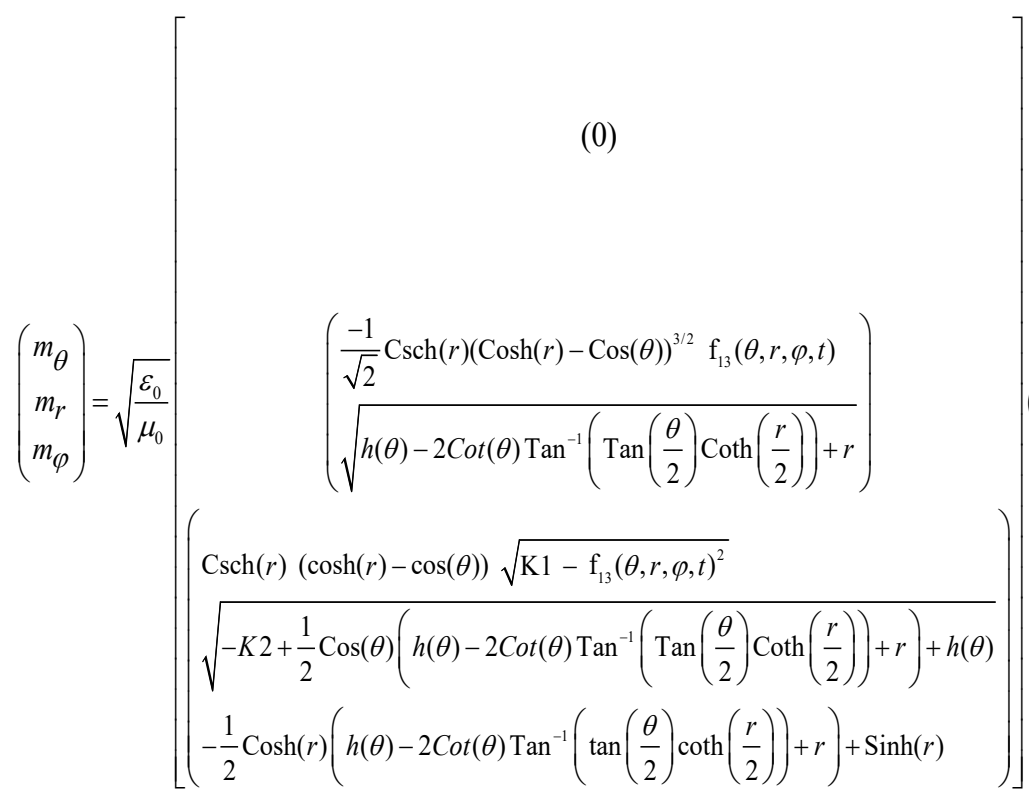




\subsection{Confined Electromagnetic Radiation within a Toroidal Coordinate System through Electromagnetic- Gravitational Interaction in a non-linear Space-Time Continuum}

The required Electromagnetic Field Configuration for a Gravitational-Electromagnetic Equilibrium in Space and Time equals in Toroidal Coordinates $\{\theta, r, \varphi, t\}$ for the Electric Field Components e $(\theta, r, \varphi, t)$ :

$$
\left.\left(\begin{array}{l}
e_{\theta} \\
e_{r} \\
e_{\varphi}
\end{array}\right)=\left[\begin{array}{c}
(0) \\
\left(\operatorname{Csch}(\mathrm{r}) \mathrm{f} 1(\theta, \mathrm{r})(\operatorname{Cosh}(\mathrm{r})-\operatorname{Cos}(\theta))^{3 / 2} \sqrt{\mathrm{K} 1-\mathrm{f} 13(\theta, \mathrm{r}, \varphi, \mathrm{t})^{2}}\right.
\end{array}\right)\right]
$$

The required Electromagnetic Field Configuration for a Gravitational-Electromagnetic Equilibrium in Space and Time equals in Toroidal Coordinates $\{\theta, r, \varphi, t\}$ for the Magnetic Field Components $\mathrm{m}(\theta, r, \varphi, t)$ :

$$
\left(\begin{array}{c}
m_{\theta} \\
m_{r} \\
m_{\varphi}
\end{array}\right)=\sqrt{\frac{\varepsilon_{0}}{\mu_{0}}}\left[\begin{array}{c}
(0) \\
\left(\operatorname{Csch}(\mathrm{r}) \mathrm{fl}(\theta, \mathrm{r})\left(-(\operatorname{Cosh}(\mathrm{r})-\operatorname{Cos}(\theta))^{3 / 2}\right) \mathrm{f} 13(\theta, \mathrm{r}, \varphi, \mathrm{t})\right) \\
\left(\begin{array}{l}
\operatorname{Csch}(\mathrm{r})(\operatorname{Cosh}(\mathrm{r})-\operatorname{Cos}(\theta)) \\
\sqrt{\operatorname{Cos}(\theta) \mathrm{fl}(\theta, \mathrm{r})^{2}-\operatorname{Cosh}(\mathrm{r}) \mathrm{fl}(\theta, \mathrm{r})^{2}-\mathrm{g}(\mathrm{r})} \sqrt{\mathrm{K} 1-\mathrm{f} 13(\theta, \mathrm{r}, \varphi, \mathrm{t})^{2}}
\end{array}\right)
\end{array}\right]
$$

The toroidal electromagnetic field configuration is in perfect equilibrium with itself and its surrounding in respectively the 198 
$\theta$ - and the $\varphi$ - direction. There is a resulting electromagnetic outward bounding force density in the r-direction, $\vec{f}(\theta, r, \varphi, t)$ indicated as the outward bounding radiation pressure of the toroidal electromagnetic confinement.

$$
\vec{f}(\theta, r, \varphi, t)=\left[\begin{array}{c}
0 \\
-\frac{\varepsilon_{0} \mathrm{~K}_{1} \operatorname{Csch}^{2}(\mathrm{r})(\operatorname{Cos}(\theta)-\operatorname{Cosh}(\mathrm{r}))^{3}\left(4 \mathrm{fl}(\theta, \mathrm{r}) \mathrm{fl} l^{(0.1)}(\theta, \mathrm{r})(\operatorname{Cos}(\theta)-\operatorname{Cosh}(\mathrm{r}))-\mathrm{g}^{\prime}(\mathrm{r})\right)}{2 \mathrm{R}} \\
0
\end{array}\right]
$$

This resulting outward bounding radiation pressure has to be compensated by the inward bounding gravitational force density, to create the required equilibrium by electromagnetic-gravitational interaction.

In a comparable way as in the example presented in spherical coordinates in (13) and (14), the electromagnetic massdensity from the energy density in the torus can be calculated. With these values the inward bounded gravitational radiation pressure can be derived. From the requirement that both force densities have to compensate each other, the Boundary Radius $R_{B O U N D A R Y}$ for the toroidal confinement can be calculated. 


\subsection{The Origin of Electromagnetic Mass (Inertia)}

When Erwin Schrödinger published in 1926 the well-known Schrödinger wave equation with the characteristic spherical and elliptical wave solutions, he found a mathematical presentation for "De Broglie-" or the Material Waves. When Bohr assigned these "De Broglie" waves as "Probability Waves", he excluded "De Broglie-Waves" from any mass. The mass was for $100 \%$ in the elementary particle itself and "De Broglie Waves" without any mass described the probability of the position of this elementary particle. A complete non logical approach.

A fundamental logical approach is to assign the mass to "De Broglie Waves". And that is only possible when we "De Broglie Waves" are just "Confined Single Harmonic Electromagnetic Waves" (confined light). For this reason the mass of confined electromagnetic radiation has to be calculated. It will be clear that it is impossible to assign mass to free electromagnetic radiation. Because it is impossible to accelerate free electromagnetic radiation and the concept of mass if based on the inertia of mass by Newton's second law. For this reason the inertia of "Confined Single Harmonic Electromagnetic Waves" (confined light) has to be calculated.

We measure the mass in $[\mathrm{kg}]$ by acceleration of the object according Newton's second law of motion. In the theory of special relativity, the speed of light is a fundamental constant. But the intensity of the light will not be constant. Speed is relative. When we emit by a laser a beam of light, the 
intensity of the light will not change with the distance (without any divergence of the beam).

However there is a difference. When the speed of the observer has the same speed as the speed of the light source, then the observer and the light source are relative at rest. And the same light intensity will be measured at the location of the emitter and at the location of the observer.

When the observer moves towards the emitter, the intensity of the light at the location of the observer will increase with $\gamma(1+\mathrm{v} / \mathrm{c})$ according the Lorentz transformation in which " $\mathrm{v}$ " is the relative velocity between emitter and observer. At low velocities the term $\gamma$ will equal 1 .

When the observer moves away from the emitter, the intensity of the light at the location of the observer will decrease with $\gamma(1-\mathrm{v} / \mathrm{c})$ according the Lorentz transformation. At low velocities the term $\gamma$ will equal 1 .

When light is confined between two $100 \%$ reflecting mirrors, then we can conclude that the speed of both mirrors will always be equal, relative to each other. And at uniform speed, the radiation pressures on both mirrors will be equal and both opposite directed radiation pressures will neutralize.

During acceleration, it will take time for the light to travel with the speed of light between both mirrors. When we consider one mirror as the emitter and the opposite mirror as the observer, we can conclude that the speed of the emitter will be different (when the beam of light leaves the emitter) than the speed of the observer (when the beam of light reaches the observer) because of the time interval, needed for 201 
the beam of light to propagate from emitter to observer during the acceleration.

During the acceleration, both opposite oriented radiation pressures on both mirrors will not be equal anymore and they will not neutralize each other anymore. During acceleration, there will be a resulting force according Newton's second law of motion caused by both different radiation pressures.

For the first step in this calculation an imaginary experiment has been used. Two $100 \%$ reflecting mirrors B and A (both in the $\mathrm{x}-\mathrm{y}$ plane and without any mass) are placed opposite each other at a distance $\Delta x$ ( ref 29, page 7, figure 1$)$. A single harmonic electromagnetic wave has been confined between both mirrors. Between both mirrors a "Standing Electromagnetic Wave" appears which has been formed by two waves travelling in opposite directions along the z-axis.

The Poynting vector corresponding with the electromagnetic wave propagating along the $\mathrm{z}$-axis in the + direction (positive direction of the z-axis) has been indicated as $\overline{\mathrm{S}}^{+}=\overline{\mathrm{E}}^{+} \times \overline{\mathrm{H}}^{+}$ and the Poynting vector corresponding with the electromagnetic wave propagating along the z-axis in the direction (opposite direction) has been indicated as $\bar{S}^{-}=\overline{\mathrm{E}}^{-} \times \overline{\mathrm{H}}^{-}$.

The system is at rest. The radiation pressures, caused by the confined electromagnetic radiation, on both mirrors A and $\mathrm{B}$ are opposite and equal in magnitude: 


$$
\mathrm{P}_{\mathrm{A}}=\frac{2 \mathrm{~S}_{\mathrm{A}}}{\mathrm{c}}=\frac{2 \mathrm{~S}_{\mathrm{B}}}{\mathrm{c}}=\mathrm{P}_{\mathrm{B}}
$$

Einstein has formulated this very well. "Velocities are always relative" . To calculate the radiation pressure on Mirror A, the velocities, only relative to Mirror A for the waves with the respective Poynting vectors $\bar{S}^{+}=\overline{\mathrm{E}}^{+} \times \overline{\mathrm{H}}^{+}$and $\bar{S}^{-}=\overline{\mathrm{E}}^{-} \times \overline{\mathrm{H}}^{-}$, have to be calculated. 


\section{3,17 The radiation pressure on Mirror A, when Mirror A moves with a velocity $v$ in the direction of the positive $z$ - axis}

When the system of "Two Mirrors B - A" moves in the direction of the positive z-axis, Mirror A moves in the direction of the positive $\mathrm{z}$-axis and the Poynting vector $\bar{S}^{+}=\overline{\mathrm{E}}^{+} \times \overline{\mathrm{H}}^{+}$will decrease according the Lorentz transformation ( ref. 29, page 23, A-57 ).

$$
\bar{S}_{\mathrm{v}}^{+}=\overline{\mathrm{E}}_{\mathrm{v}}^{+} \times \overline{\mathrm{H}}_{\mathrm{v}}^{+}=\gamma^{2}\left(1-\frac{\mathrm{v}}{\mathrm{c}}\right)^{2}\left(\overline{\mathrm{E}}^{+} \times \overline{\mathrm{H}}^{+}\right)
$$

When the system of "Two Mirrors B - A" moves in the direction of the positive z-axis, Mirror A moves in the direction of the positive $\mathrm{Z}$-axis the Poynting vector $\bar{S}^{-}=\overline{\mathrm{E}}^{-} \times \overline{\mathrm{H}}^{-}$will increase according the Lorentz transformation (ref. 29, page 222, A-57).

$$
\bar{S}_{\mathrm{v}}^{-}=\overline{\mathrm{E}}_{\mathrm{v}}^{-} \times \overline{\mathrm{H}}_{\mathrm{v}}^{-}=\gamma^{2}\left(1+\frac{\mathrm{v}}{\mathrm{c}}\right)^{2}\left(\overline{\mathrm{E}}^{+} \times \overline{\mathrm{H}}^{+}\right)
$$

The total radiation pressure, caused by the confined electromagnetic radiation, on mirror A equals:

$$
\mathrm{P}_{\mathrm{A}}=\frac{\mathrm{S}_{A}^{+}+\mathrm{S}_{A}^{-}}{\mathrm{c}}=\frac{\gamma^{2}\left(\left(1-\frac{\mathrm{v}}{\mathrm{c}}\right)^{2}+\left(1+\frac{\mathrm{v}}{\mathrm{c}}\right)^{2}\right)\left(\overline{\mathrm{E}}^{+} \times \overline{\mathrm{H}}^{+}\right)}{\mathrm{c}}
$$




\subsection{The radiation pressure on Mirror B when Mirror B moves with a velocity $v$ in the direction of the positive $z$ - axis}

When the system of "Two Mirrors B - A" moves in the direction of the positive z-axis, Mirror B moves in the direction of the positive $\mathrm{z}$-axis and the Poynting vector $\bar{S}^{-}=\overline{\mathrm{E}}^{-} \times \overline{\mathrm{H}}^{-}$will increase according the Lorentz transformation ( ref. 29, page 23, A-57 ).

$$
\bar{S}_{\mathrm{v}}^{-}=\overline{\mathrm{E}}_{\mathrm{v}}^{-} \times \overline{\mathrm{H}}_{\mathrm{v}}^{-}=\gamma^{2}\left(1+\frac{\mathrm{v}}{\mathrm{c}}\right)^{2}\left(\overline{\mathrm{E}}^{+} \times \overline{\mathrm{H}}^{+}\right)
$$

When the system of "Two Mirrors B - A" moves in the direction of the positive z-axis, Mirror A moves in the direction of the positive $\mathrm{z}$-axis the Poynting vector $\overline{\mathrm{S}}^{+}=\overline{\mathrm{E}}^{+} \times \overline{\mathrm{H}}^{+}$will increase according the Lorentz

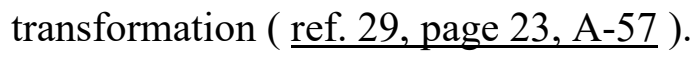

$$
\bar{S}_{\mathrm{v}}^{+}=\overline{\mathrm{E}}_{\mathrm{v}}^{+} \times \overline{\mathrm{H}}_{\mathrm{v}}^{+}=\gamma^{2}\left(1-\frac{\mathrm{v}}{\mathrm{c}}\right)^{2}\left(\overline{\mathrm{E}}^{+} \times \overline{\mathrm{H}}^{+}\right)
$$

The total radiation pressure, caused by the confined electromagnetic radiation, on mirror $B$ equals: 


$$
\mathrm{P}_{\mathrm{A}}=\frac{\mathrm{S}_{A}^{+}+\mathrm{S}_{A}^{-}}{\mathrm{c}}=\frac{\gamma^{2}\left(\left(1+\frac{\mathrm{v}}{\mathrm{c}}\right)^{2}+\left(1-\frac{\mathrm{v}}{\mathrm{c}}\right)^{2}\right)\left(\overline{\mathrm{E}}^{+} \times \overline{\mathrm{H}}^{+}\right)}{\mathrm{c}}
$$

$\mathrm{P}_{\mathrm{A}}$ and $\mathrm{P}_{B}$ are still equal in magnitude and both in opposite direction and still cancel each other. The system fulfils Newton's first law of motion. 


\subsection{Newton's second Law of Motion (Inertia) for Confined Electromagnetic Radiation}

When the system of "Two Mirrors B - A" accelerates, the velocity increases with $\Delta \mathrm{v}$ in a time interval $\Delta \mathrm{t}$. At time $\mathrm{t}$ the radiation pressures on mirror $\mathrm{A}$ and mirror $\mathrm{B}$ are presented in (29) and (32). At time $t+\Delta t$ the radiation pressures on Mirror A and Mirror B will different:

The radiation pressure at time $\mathrm{t}+\Delta \mathrm{t}$ caused by the confined electromagnetic radiation, on mirror A equals:

$$
\mathrm{P}_{\mathrm{A}}=\frac{\mathrm{S}_{A}^{+}+\mathrm{S}_{A}^{-}}{\mathrm{c}}=\frac{\gamma^{2}\left(\left(1+\frac{(\mathrm{v})}{\mathrm{c}}\right)^{2}+\left(1-\frac{(\mathrm{v}+\Delta \mathrm{v})}{\mathrm{c}}\right)^{2}\right)\left(\overline{\mathrm{E}}^{+} \times \overline{\mathrm{H}}^{+}\right)}{\mathrm{c}}
$$

Because the wave with Poynting vector $\overline{\mathrm{S}}^{+}=\overline{\mathrm{E}}^{+} \times \overline{\mathrm{H}}^{+}$has left Mirror B at " $\mathrm{t}$ " and during the time interval $\Delta \mathrm{t}$ the magnitude of $\overline{\mathrm{E}}_{\mathrm{t}}^{+}=\left(1+\frac{\mathrm{v}}{\mathrm{c}}\right) \overline{\mathrm{E}}^{+}$and $\overline{\mathrm{H}}_{\mathrm{t}}^{+}=\left(1+\frac{\mathrm{v}}{\mathrm{c}}\right) \overline{\mathrm{H}}^{+}$has not changed.

The radiation pressure at time $\mathrm{t}+\Delta \mathrm{t}$ caused by the confined electromagnetic radiation, on mirror $B$ equals:

$$
\mathrm{P}_{B}=\frac{\mathrm{S}_{B}^{+}+\mathrm{S}_{B}^{-}}{\mathrm{c}}=\frac{\gamma^{2}\left(\left(1+\frac{(\mathrm{v}+\Delta \mathrm{v})}{\mathrm{c}}\right)^{2}+\left(1-\frac{(\mathrm{v})}{\mathrm{c}}\right)^{2}\right)\left(\overline{\mathrm{E}}^{+} \times \overline{\mathrm{H}}^{+}\right)}{\mathrm{c}}
$$


Because the wave with Poynting vector $\bar{S}^{-}=\overline{\mathrm{E}}^{-} \times \overline{\mathrm{H}}^{-}$has left Mirror A at " $\mathrm{t}$ " and during the time interval $\Delta \mathrm{t}$ the magnitude of ${\overline{\mathrm{E}_{\mathrm{t}}^{-}}}^{-}=\left(1+\frac{\mathrm{v}}{\mathrm{c}}\right) \overline{\mathrm{E}}^{-}$and $\overline{\mathrm{H}}_{\mathrm{t}}^{-}=\left(1+\frac{\mathrm{v}}{\mathrm{c}}\right) \overline{\mathrm{H}}^{-}$has not changed.

The radiation pressures on Mirror A and Mirror B do not counterbalance each other anymore and the resulting radiation pressure equals:

$$
\mathrm{P}_{\mathrm{B}}-\mathrm{P}_{\mathrm{A}}=\frac{\gamma^{2}(4 \Delta \mathrm{v}) \mathrm{S}}{\mathrm{c}^{2}}
$$

Equation (35) can be written as:

$$
\mathrm{P}_{\mathrm{B}}-\mathrm{P}_{\mathrm{A}}=\frac{\gamma^{2}(4 \Delta \mathrm{v}) \mathrm{S}}{\mathrm{c}^{2}}=\frac{\gamma^{2}\left(4 \frac{\Delta \mathrm{v}}{\Delta \mathrm{t}}\right) \mathrm{S} \Delta \mathrm{t}}{\mathrm{c}^{2}}=\gamma^{2} \frac{\mathrm{W}}{\mathrm{c}^{2}} \mathrm{a}=\gamma^{2} \mathrm{ma}
$$

In which the acceleration $\mathrm{a}=\frac{\Delta \mathrm{v}}{\Delta \mathrm{t}}$ and the inertia $\mathrm{m}=\frac{\mathrm{W}}{\mathrm{c}^{2}}$. At non relativistic velocities $\gamma=1$ and (36) turns into the Newton's second law of motion. From (36) also Einstein's famous equation $\mathrm{W}=\mathrm{m} \mathrm{c}^{2}$ follows. In (36) $\mathrm{W}$ is the total confined electromagnetic mass. 
Now we can consider electromagnetic confinements without mirrors but electromagnetic confinements through electromagnetic gravitational interaction. By superposition and integration over arbitrary surfaces it is possible to prove that all confined electromagnetic radiation equals (36) and has electromagnetic mass and follow Newton's second law of motion. 


\subsection{Quantum Mechanical Entanglement}

One of the first answers every Quantum Physicist will give you when you doubt the Holy Grail of the "Particle-Wave Duality" in Quantum Physics is Bell's Theorem. But the "Particle-Wave Duality" can never explain the laws in Quantum Mechanics. In special the "Particle-Wave Duality" cannot explain "Entanglement".

In the new model of the "Particle-Wave-Mass" Tri-Unity , the concept of a particle on itself has completely disappeared. A particle itself does not exist anymore. The "Particle-WaveMass" is a wave of confined light (Electromagnetic Radiation) with a "particle aspect" and a "mass aspect". And sometimes we observe only one of the three aspects.

To demonstrate this, a simple model of a string in a music instrument like a piano, will be used. As soon the string has been excited, we will hear a tune (frequency spectrum). Along the string a pattern of "standing (stationary) transversal waves" occurs. When we observe one single harmonic frequency we observe that along the string a pattern occurs of nodes and anti-nodes occurs. The nodes as well as the anti-nodes are connected together in an "Entanglement" And because the information travels along the string simultaneously in both directions, it gives the impression that the information travels with an infinite speed. Because the sound wave in the string has been confined between the two ends of the string, Entanglement occurs.

Entanglement is only possible within the confinement of wave patterns. Because only then the wave pattern can mathematically be considered as the superposition of waves propagating in opposite directions and forming "standing (stationary) waves" It does not matter if the "standing 
(stationary) waves" occur because of the confinement by the ends of the string or a confinement of an electromagnetic field due to "Electromagnetic-Gravitational Interaction".

Now we consider the creation of an "electron-anti electron" pair. Both have been created at the same location. That is the exact location where the string has been excited in the example. When the electron-anti electron pair moves away from each other, it is still in the essence "one confined wave pattern". And only through Gravitational-Electromagnetic Interaction this wave pattern has been confined and the area of confinement becomes only larger when both particles move apart. The electron/anti-electron pair is still one stationary confined harmonic electromagnetic wave pattern, which is the superposition of a complex nodes-anti nodes pattern. Confined by its own "Electromagnetic-Gravitational Field "Entanglement" will occur in a comparable way how it occurs in the string of a music instrument. Confinement is the necessary requirement for the existence of "Entanglement".

Entanglement is the fundamental evidence that the "ParticleWave Duality" in Classical Quantum Mechanics is not able to explain "Entanglement". Particles can not be at the same place simultaneously. The weakness in Classical Quantum Mechanics is the "particle". Not the "wave". Modern experiments crumble down the "Particle-Wave-Duality" model of Niels Bohr more and more. How is it possible to make a photo of a complex probability wave? How is "Entanglement" possible? How can a photon create an electron/anti-electron pair?

Classical Quantum Physics needs a make-over. A new concept that does not crumble down further by every new experiment. And the particle concept is the weakness in Classical Quantum Mechanics. We come closer and closer to the final conclusion that particles do not exist. "Elementary 211 
particles" is a concept that worked very well for the last 2000 years. But it does no work anymore. We have to give up a way of thinking in the way we are already thinking for more than 2000 years since Plato introduced the concept of "Elementary particle".

Times are changing and it is time for us to leave the ancient save roads of the Greek Philosophers and give up the safety of our illusions. It is time to move on and let the "particle" go.

Let us build a new model. A new model without particles. A model free from the ancient Greek Philosophers. A model just grounded only on the wave.

And that is what I am offering. A new model without particles. In which the property of matter will be carried only by the wave. And the wave is already given to us since the creation of our Universe. The Light Wave. The Light Wave which carries the ability to confine itself and manifest itself like a particle. The Light wave which carries the ability to confine itself and manifest itself in the property of inertia and carries mass. The confined light wave that is in fundamental essence just only a wave but carries the 3-unity in itself of particle -wave - mass. The Particle-Wave-Mass 3-Unity. 


\subsection{A Gravitational-Electromagnetic model beyond the Superstring}

Calculations in Mathematica 11.0 .nb file and in PDF file.

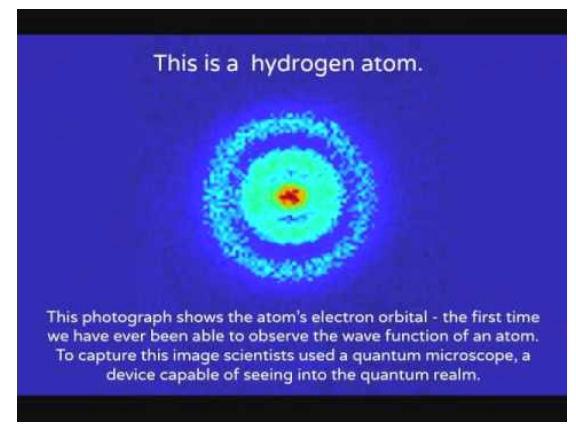

Figure 11 First Image of the Hydrogen Atom's Orbital structure

"De Broglie Waves" are real and do exist. Schrödinger" and as well Dirac both have written the simple well-known (electromagnetic) Continuity Equation (ref. 29, page 208, equation 57). in a complex form. What they have really found are electromagnetic waves. Single harmonic and confined. The Schrödinger Solution of a Spherical Probability wave around the nucleus has been interpreted wrong. It is not a complex probability wave. It is a real electromagnetic wave in which the real part is the solution for the electric part and the imaginary part is the magnetic part, just written with an "i" index before the term. The quantum mechanical wave function $\psi$ is in real a vector function $\bar{\phi}$ which equals:

$\bar{\phi}=\frac{1}{\sqrt{2 \mu}}\left(\overline{\mathrm{B}}+\mathrm{i} \frac{\overline{\mathrm{E}}}{c}\right)$ 
and the complex conjugated vector function equals:

$$
\overline{\phi^{*}}=\frac{1}{\sqrt{2 \mu}}\left(\overline{\mathrm{B}}-\mathrm{i} \frac{\overline{\mathrm{E}}}{c}\right)
$$

And the dot product equals the electromagnetic energy density w:

$$
\bar{\phi} \cdot \overline{\phi^{*}}=\frac{1}{2 \mu}\left(\overline{\mathrm{B}}+\mathrm{i} \frac{\overline{\mathrm{E}}}{c}\right) \cdot\left(\overline{\mathrm{B}}-\mathrm{i} \frac{\overline{\mathrm{E}}}{c}\right)=\frac{1}{2} \mu \mathrm{H}^{2}+\frac{1}{2} \varepsilon \mathrm{E}^{2}=\mathrm{w}
$$

The cross product is proportional to the Poynting vector (ref. 29, page 202, equation 15 ).

$\bar{\phi} \times \overline{\phi^{*}}=\frac{1}{2 \mu}\left(\overline{\mathrm{B}}+\mathrm{i} \frac{\overline{\mathrm{E}}}{c}\right) \times\left(\overline{\mathrm{B}}-\mathrm{i} \frac{\overline{\mathrm{E}}}{c}\right)=\mathrm{i} \sqrt{\varepsilon \mu} \overline{\mathrm{E}} \times \overline{\mathrm{H}}=\mathrm{i} \sqrt{\varepsilon \mu} \overline{\mathrm{S}}$

The Gravitational-Electromagnetic Confinement for the elementary structure beyond the "superstring" is presented in equation (5-a).

$$
\begin{gathered}
-\frac{1}{c^{2}} \frac{\partial(\bar{E} \times \bar{H})}{\partial t}+\varepsilon_{0} \bar{E}(\nabla \cdot \bar{E})-\varepsilon_{0} \bar{E} \times(\nabla \times \bar{E})+\mu_{0} \bar{H}(\nabla \cdot \bar{H})- \\
-\mu_{0} \bar{H} \times(\nabla \times \bar{H})-\frac{1}{2} \varepsilon_{0}^{2} \mu_{0}(\bar{E} \cdot \bar{E}) \overline{\mathrm{g}}-\frac{1}{2} \varepsilon_{0} \mu_{0}{ }^{2}(\bar{H} \cdot \bar{H}) \overline{\mathrm{g}}=\overline{0}
\end{gathered}
$$

In which $\bar{g}$ represents the (radial oriënted) gravitational acceleration caused by the electromagnetic mass density of the confined electromagnetic radiation. 
The solution for equation (5-a) equals:

$$
\begin{aligned}
& \left(\begin{array}{l}
e_{r} \\
e_{\theta} \\
e_{\varphi}
\end{array}\right)=\left(\begin{array}{c}
0 \\
\mathrm{f}(\mathrm{r}) \operatorname{Sin}(\omega \mathrm{t}) \\
-\mathrm{f}(\mathrm{r}) \operatorname{Cos}(\omega \mathrm{t})
\end{array}\right) \quad\left(\begin{array}{l}
m_{r} \\
m_{\theta} \\
m_{\varphi}
\end{array}\right)=\left(\begin{array}{c}
0 \\
\mathrm{f}(\mathrm{r}) \operatorname{Cos}(\omega \mathrm{t}) \\
\mathrm{f}(\mathrm{r}) \operatorname{Sin}(\omega \mathrm{t})
\end{array}\right) \quad \bar{g}=\left(\begin{array}{c}
\frac{G_{1}}{4 \pi \mathrm{r}^{2}} \\
0 \\
0
\end{array}\right) \\
& \mathrm{w}_{\mathrm{em}}=\left(\frac{\mu_{0}}{2}(\overline{\mathrm{m}} \cdot \overline{\mathrm{m}})+\frac{\varepsilon_{0}}{2}(\overline{\mathrm{e}} \cdot \overline{\mathrm{e}})\right)=\varepsilon_{0} \mathrm{f}(r)^{2}
\end{aligned}
$$

In which $\mathrm{f}(\mathrm{r})$ equals:

$$
f[r]=K \mathrm{e}^{-\frac{G 1 \varepsilon_{0} \mu_{0}}{r}+8 \pi \log [r]}
$$




\subsection{A Gravitational-Electromagnetic Confinement} Type 1

$\left(\mathrm{emm}=10^{-4}[\mathrm{~kg}] ;\right.$ radius $\left.=2 \times 10^{-35}[\mathrm{~m}]\right):$

The chosen values equal:

$$
\begin{aligned}
& f[r]=K \mathrm{e}^{-\frac{-\frac{G \mathrm{emm} \varepsilon_{0} \mu_{0}}{r}+8 \pi \log [r]}{8 \pi}} \\
& G 1=6.6740810^{-11} \\
& \mathrm{emm}=10^{-4} \\
& \varepsilon_{0}=8.8510^{-12} \\
& \mu_{0}=1.256637061435917210^{-6}
\end{aligned}
$$

In which "emm" equals the electromagnetic mass of the confinement located at the center according Newton's Shell Theorem.

For an electromagnetic mass of the confinement type (1): $\mathrm{emm}=10^{-4}[\mathrm{~kg}]$, the radius of the confinement equals approximately $2 \times 10^{-35}[\mathrm{~m}]$. This is in the order of Planck's Length,

The Plot graph of the Electric Field Intensity $\mathrm{f}(\mathrm{r})$ of the confinement has been presented as a function of the radius in figure (7) and figure (8): 


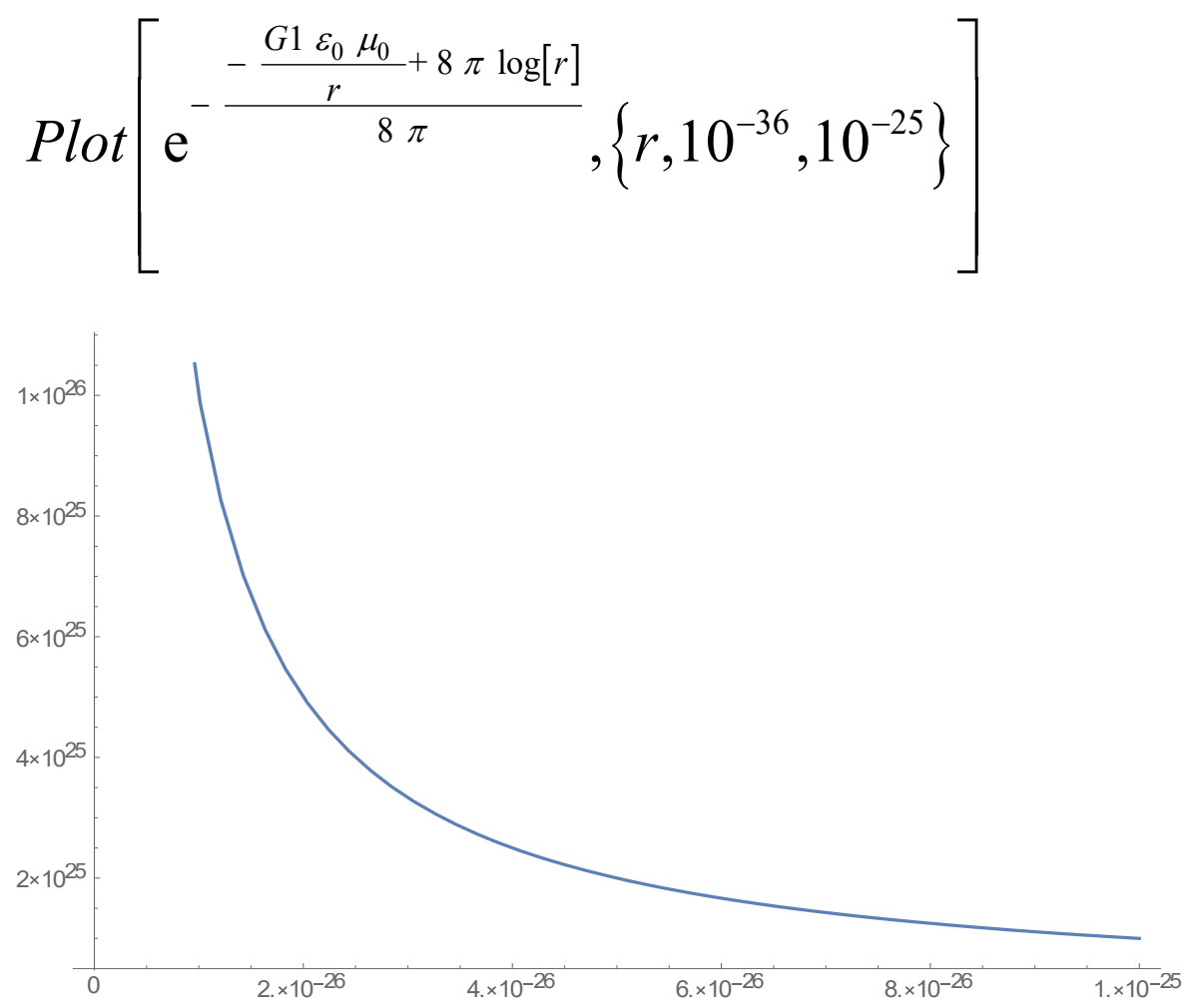

Figure 12 PlotGraph of the Electric Field Intensity $\mathrm{f}(\mathrm{r})$ for the region $10^{-36}<\mathbf{r}<10^{-25}$ in which the gravitational field acceleration has been chosen accordingly an electromagnetic mass of $10^{-4}[\mathrm{~kg}]$ located at the center of the confinement, according Newton's Shell Theorem. 


$$
\operatorname{Plot}\left[\mathrm{e}^{-\frac{-\frac{G 1 \varepsilon_{0} \mu_{0}}{r}+8 \pi \log [r]}{8 \pi}},\left\{r, 10^{-36}, 10^{-35}\right\}\right]
$$

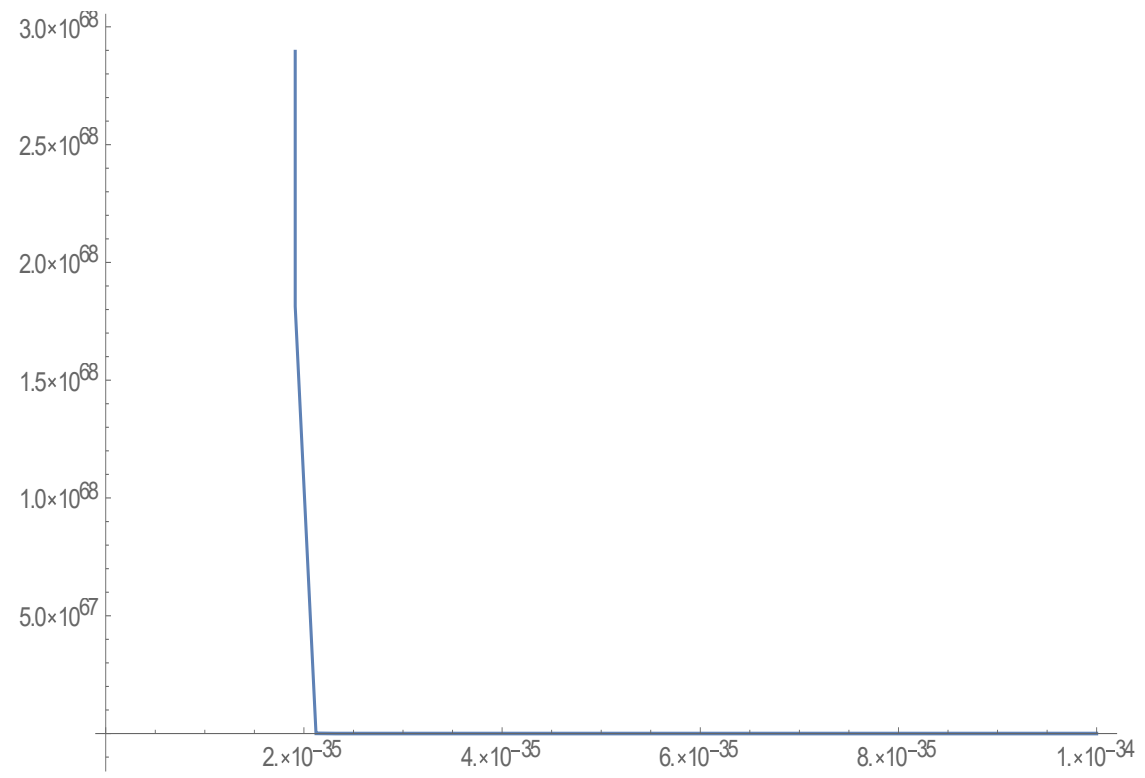

Figure 13 PlotGraph of the Electric Field Intensity $\mathrm{f}(\mathrm{r})$ for the region $10^{-36}<\mathbf{r}<\mathbf{1 0}^{-35}$ in which the gravitational field acceleration has been chosen accordingly an electromagnetic mass of $10^{-4}[\mathrm{~kg}]$ located at the center of the confinement, according Newton's Shell Theorem. 
It follows from Figure 8 that the radius of the stable gravitational electromagnetic confinement equals approximately $2 \times 10^{-35}[\mathrm{~m}]$, which is the size of the Planck length. According the theory of superstrings, the fundamental constituents of reality are strings of the Planck length (about $\left.1.6210^{-35}[\mathrm{~m}]\right)$ that vibrate at resonant frequencies. 


\subsection{A Gravitational-Electromagnetic Confinement Type $2\left(\mathrm{emm}=10^{-12}[\mathrm{~kg}]\right.$; radius $\left.=2.5 \times 10^{-43}[\mathrm{~m}]\right)$ :}

For an electromagnetic mass of the confinement type (2): $\mathrm{emm}=10^{-12}[\mathrm{~kg}]$, the radius of the confinement equals approximately $2.5 \times 10^{-43}[\mathrm{~m}]$. This is much smaller than Planck's Length and has been indicated as "sub Planck Length". 
The Plot graph of the Electric Field Intensity $f(r)$ of the confinement has been presented as a function of the radius in figure (9)_ and figure (10):

$$
\operatorname{Plot}\left[\mathrm{e}^{-\frac{-\frac{G 1 \varepsilon_{0} \mu_{0}}{r}+8 \pi \log [r]}{8 \pi}},\left\{r, 10^{-43}, 10^{-40}\right\}\right]
$$

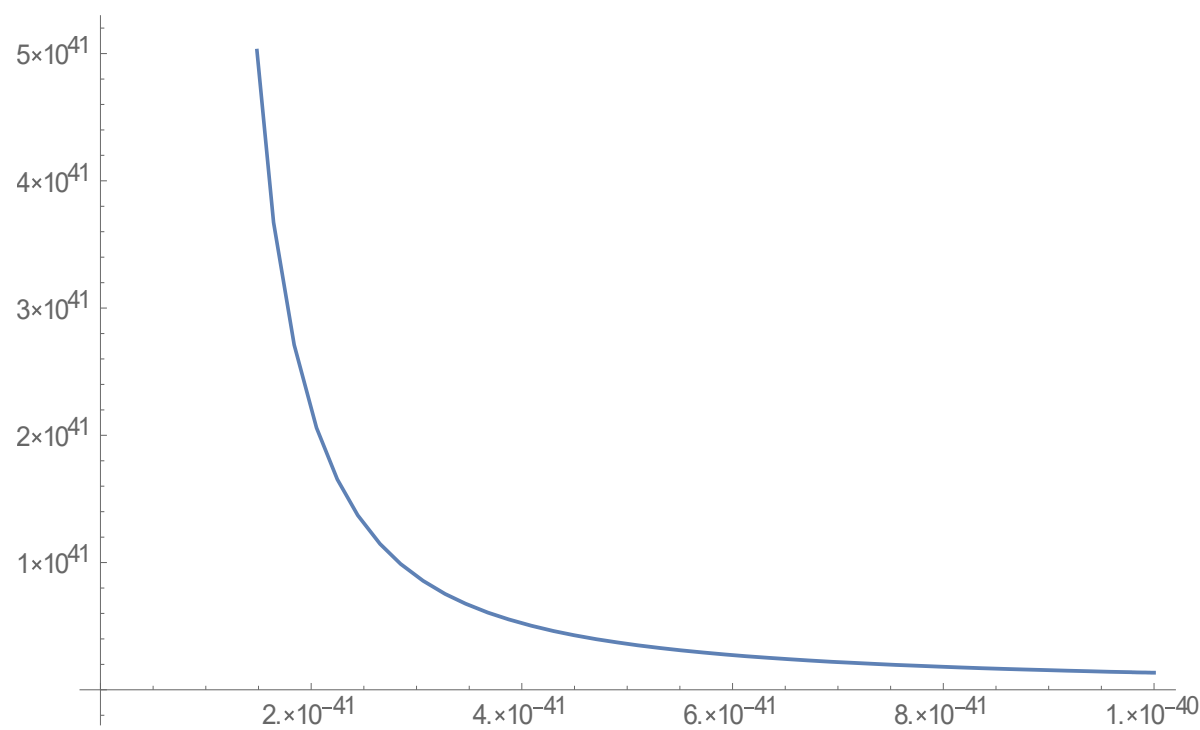

Figure 14 PlotGraph of the Electric Field Intensity $\mathrm{f}(\mathrm{r})$ for the region $10^{-36}<\mathrm{r}<\mathbf{1 0}^{-25}$ in which the gravitational field acceleration has been chosen accordingly an electromagnetic mass of $10^{-4}[\mathrm{~kg}]$ located at the center of the confinement, according Newton's Shell Theorem. 


$$
\operatorname{Plot}\left[\mathrm{e}^{-\frac{-\frac{G 1 \varepsilon_{0} \mu_{0}}{r}+8 \pi \log [r]}{8 \pi}},\left\{r, 10^{-43}, 10^{-42}\right\}\right]
$$

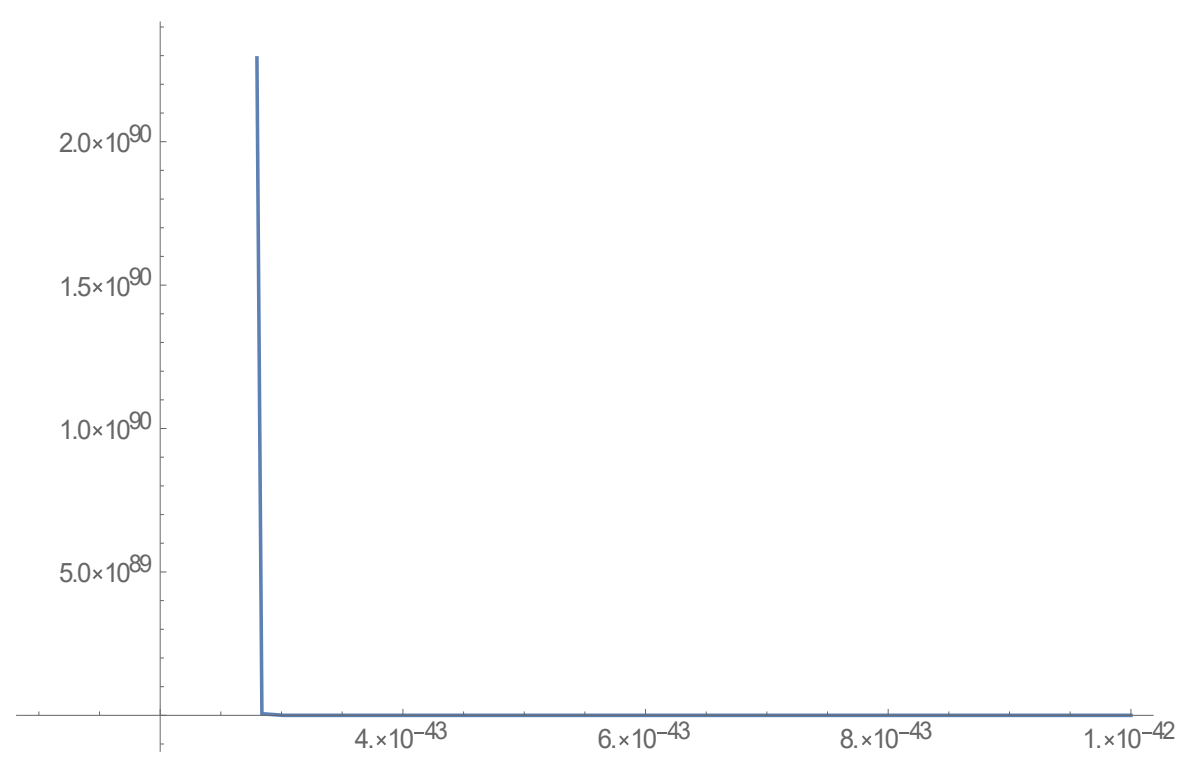

Figure 15 PlotGraph of the Electric Field Intensity $\mathrm{f}(\mathrm{r})$ for the region $10^{-43}<r<10^{-42}$ in which the gravitational field acceleration has been chosen accordingly an electromagnetic mass of $10^{-12}[\mathrm{~kg}]$ located at the center of the confinement, according Newton's Shell Theorem. 


\subsection{A Gravitational-Electromagnetic Confinement Type $3\left(\mathrm{emm}=1.6726 \times 10^{-27}[\mathrm{~kg}]\right.$; radius $\left.=3 \times 10^{-58}[\mathrm{~m}]\right)$ :}

For an electromagnetic mass of the confinement type (3): $\mathrm{emm}=1.6726 \times 10^{-27}[\mathrm{~kg}]$ (mass of proton), the radius of the confinement equals approximately $3 \times 10^{-58}[\mathrm{~m}]$. This is far beyond the order of Planck's Length,

The Plot graph of the Electric Field Intensity $\mathrm{f}(\mathrm{r})$ of the confinement has been presented as a function of the radius in figure (11) and figure (12): 


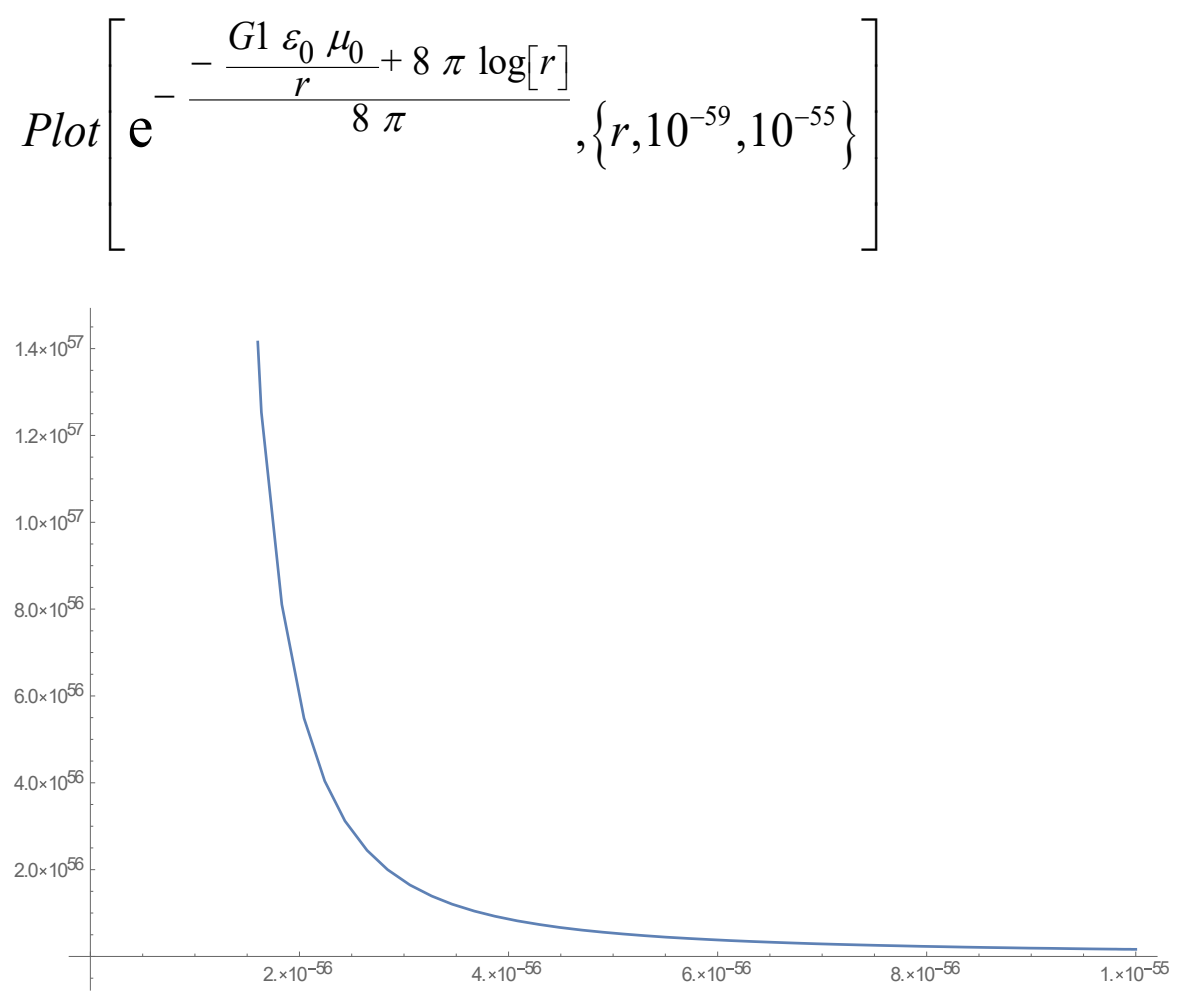

Figure 16 PlotGraph of the Electric Field Intensity $\mathrm{f}(\mathrm{r})$ for the region $10^{-59}<\mathrm{r}<10^{-55}$ in which the gravitational field acceleration has been chosen accordingly an electromagnetic mass of $1.6726 \times 10^{-27}$ [kg] located at the center of the confinement, according Newton's Shell Theorem. 


$$
\text { Plot }\left[\mathrm{e}^{-\frac{-\frac{G 1 \varepsilon_{0} \mu_{0}}{r}+8 \pi \log [r]}{8 \pi}},\left\{r, 10^{-59}, 10^{-57}\right\}\right]
$$

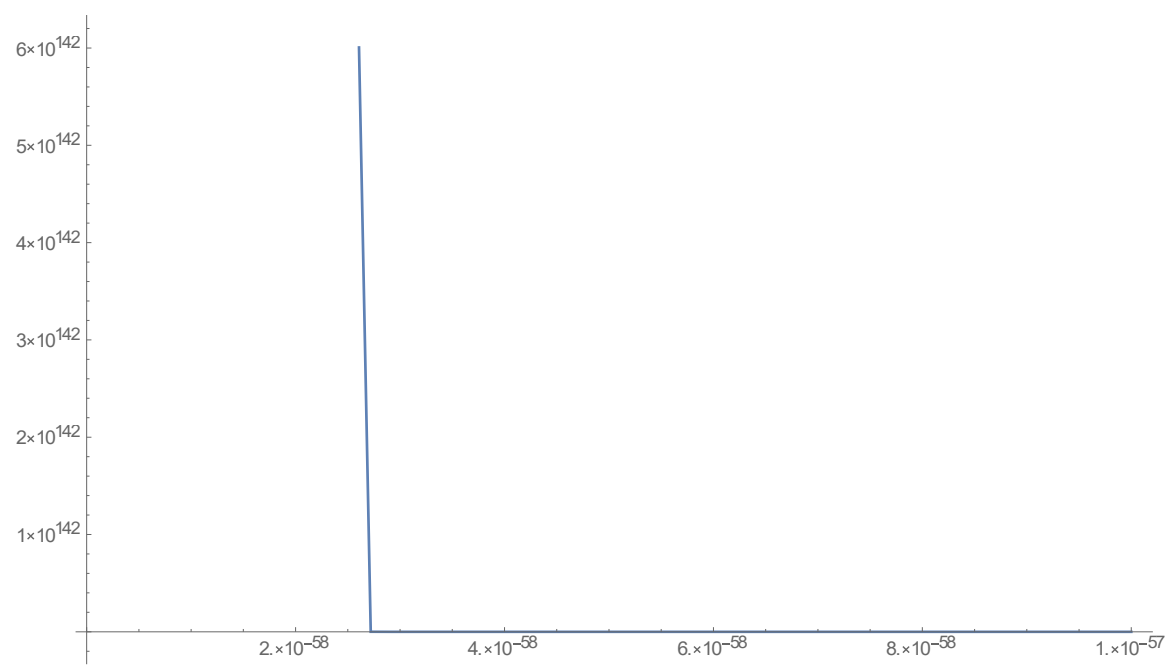

Figure 17 PlotGraph of the Electric Field Intensity $\mathbf{f}(\mathbf{r})$ for the region $10^{-59}<\mathbf{r}<10^{-57}$ in which the gravitational field acceleration has been chosen accordingly an electromagnetic mass of $1.6726 \times 10^{-27}[\mathrm{~kg}]$ located at the center of the confinement, according Newton's Shell Theorem.

The fundamental question is: How it is possible to create confinements from "visible light" (with a wave length between $3.9 \times 10^{-7}[\mathrm{~m}]$ until $7 \times 10^{-7}[\mathrm{~m}]$ ) within dimensions smaller than Planck's Length?

This is only possible when the wave length of the confined radiation is smaller than de dimensions of the confinement. 
This requires extreme high frequencies. The transformation in frequency from visible light into the extreme high frequency of the confinement is possible because of the Lorentz transformation during the collapse of the radiation when the confinement has been formed (implosion of visible light).

\subsection{The Origin of Electric Charge and Magnetic Spin in discrete values}

The Gravitational-Electromagnetic Confinement for the elementary structure of the "superstring" is presented in equation (5-a).

$$
\begin{gathered}
-\frac{1}{c^{2}} \frac{\partial(\bar{E} \times \bar{H})}{\partial t}+\varepsilon_{0} \bar{E}(\nabla \cdot \bar{E})-\varepsilon_{0} \bar{E} \times(\nabla \times \bar{E})+\mu_{0} \bar{H}(\nabla \cdot \bar{H})- \\
-\mu_{0} \bar{H} \times(\nabla \times \bar{H})-\frac{1}{2} \varepsilon_{0}^{2} \mu_{0}(\bar{E} \cdot \bar{E}) \overline{\mathrm{g}}-\frac{1}{2} \varepsilon_{0} \mu_{0}^{2}(\bar{H} \cdot \bar{H}) \overline{\mathrm{g}}=\overline{0}
\end{gathered}
$$

In which $\bar{g}$ represents the (radial oriented) gravitational acceleration caused by the electromagnetic mass density of the confined electromagnetic radiation.

To find the origin of Electric Charge and Magnetic Spin we choose as an example the solution for equation (5-a) which equals:

$$
\begin{aligned}
& \left(\begin{array}{l}
e_{r} \\
e_{\theta} \\
e_{\varphi}
\end{array}\right)=\left(\begin{array}{c}
0 \\
\mathrm{f} 1(\mathrm{r}, \theta, \varphi, \mathrm{t}) \operatorname{Sin}(\omega \mathrm{t}) \\
-\mathrm{f} 2(\mathrm{r}, \theta, \varphi, \mathrm{t}) \operatorname{Cos}(\omega \mathrm{t})
\end{array}\right) \quad\left(\begin{array}{l}
m_{r} \\
m_{\theta} \\
m_{\varphi}
\end{array}\right)=\left(\begin{array}{c}
0 \\
\mathrm{f} 2(\mathrm{r}, \theta, \varphi, \mathrm{t}) \operatorname{Cos}(\omega \mathrm{t}) \\
\mathrm{f} 1(\mathrm{r}, \theta, \varphi, \mathrm{t}) \operatorname{Sin}(\omega \mathrm{t})
\end{array}\right) \\
& \mathrm{w}_{\mathrm{em}}=\left(\frac{\mu_{0}}{2}(\overline{\mathrm{m}} \cdot \overline{\mathrm{m}})+\frac{\varepsilon_{0}}{2}(\overline{\mathrm{e}} \cdot \overline{\mathrm{e}})\right)=\varepsilon_{0} \mathrm{f}(r)^{2}
\end{aligned}
$$


In which $\mathrm{f}[\mathrm{r}], f 1[r, \theta, \varphi, t], f 2[r, \theta, \varphi, t]$ equals:

$$
\begin{aligned}
& f[r]=K \mathrm{e}^{-\frac{-\frac{G 1 \varepsilon_{0} \mu_{0}}{r}+8 \pi \log [r]}{8 \pi}} \\
& f 1[r, \theta, \varphi, t]=K \mathrm{e}^{-\frac{-\frac{G 1 \varepsilon_{0} \mu_{0}}{r}+8 \pi \log [r]}{8 \pi}} g 1[\theta, \varphi, t] \\
& f 2[r, \theta, \varphi, t]=\frac{K \mathrm{e}^{-\frac{-\frac{G 1 \varepsilon_{0} \mu_{0}}{r}+8 \pi \log [r]}{8 \pi}} \sqrt{-g 1[\theta, \varphi, t]^{2}+\cos [2 t \omega] g 1[\theta, \varphi, t]^{2}+2 h[\theta, \varphi]}}{\sqrt{2}}
\end{aligned}
$$

In which $g 1[\theta, \varphi, t]$ and $\mathrm{h}[\theta, \varphi]$ are arbitrary function.

The "sub Max Planck's length" confinement has been described for the electric field intensity:

$$
\left(\begin{array}{c}
e_{r} \\
e_{\theta} \\
e_{\varphi}
\end{array}\right)=\left(\begin{array}{c}
0 \\
K \mathrm{e}^{-\frac{-\frac{G 1 \varepsilon_{0} \mu_{0}}{r}+8 \pi \log [r]}{8 \pi}} \\
g 1[\theta, \varphi, t] \sin [t \omega] \\
-\frac{K \mathrm{e}^{-\frac{-\frac{G 1 \varepsilon_{0} \mu_{0}}{r}+8 \pi \log [r]}{8 \pi}} \sqrt{-g 1[\theta, \varphi, t]^{2}+\cos [2 t \omega] g 1[\theta, \varphi, t]^{2}+2 h[\theta, \varphi]}}{\sqrt{2}}
\end{array}\right)
$$

The "sub Max Planck's length" confinement has been described for the magnetic field intensity:

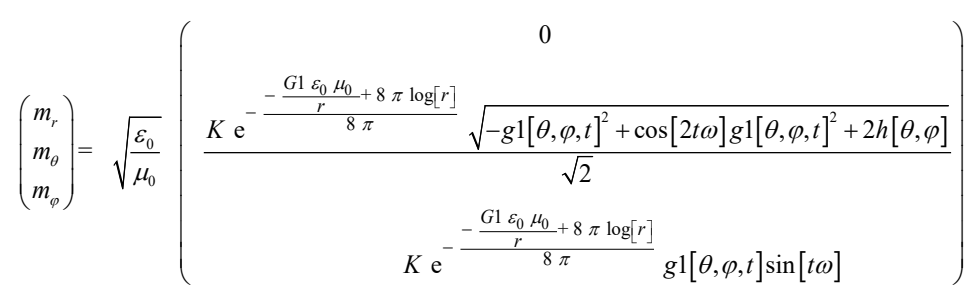




\subsection{The 5 Types of "Sub Max Planck Length \\ Gravitational-Electromagnetic Confinements" resulting in Electric Charge and Magnetic Spin}

The following functions with the quantum variables $\{\mathrm{m} 1, \mathrm{n} 1$, p1, q1\} have been chosen:

$$
\begin{aligned}
& f[r]=K \mathrm{e}^{-\frac{-\frac{G 1 \varepsilon_{0} \mu_{0}}{r}+8 \pi \log [r]}{8 \pi}} \\
& \mathrm{g} 1(\theta, \varphi, t)=\sin (t \omega)(\sin (\pi \theta \mathrm{m} 1) \sin (\mathrm{n} 12 \pi \varphi)+1) \\
& h(\theta, \varphi)=\sin (\pi \theta \mathrm{p} 1) \sin (\mathrm{q} 12 \pi \varphi)+1 \\
& \mathrm{~g} 2(\theta, \varphi, t)=\frac{\sec (t \omega) \sqrt{\cos (2 t \omega) \mathrm{g} 1(\theta, \varphi, t)^{2}-\mathrm{g} 1(\theta, \varphi, t)^{2}+2 h(\theta, \varphi)}}{\sqrt{2}} \\
& f 1[r, \theta, \varphi, t]=\mathrm{e}^{-\frac{-\frac{G 1 \varepsilon_{0} \mu_{0}}{r}+8 \pi \log [r]}{8 \pi}} K \operatorname{gl}[\theta, \varphi, t] \\
& f 2[r, \theta, \varphi, t]=\frac{\mathrm{e}^{-\frac{-\frac{G 1 \varepsilon_{0} \mu_{0}}{r}+8 \pi \log [r]}{8 \pi}} K \sqrt{-g 1[\theta, \varphi, t]^{2}+\cos [2 t \omega] g 1[\theta, \varphi, t]^{2}+2 h[\theta, \varphi]}}{\sqrt{2}}
\end{aligned}
$$




\subsubsection{Type 1 of "Sub Max Planck Length Gravitational- Electromagnetic Confinements" (Electric- and Magnetic Dipoles, Electric- and Magnetic Spin) $\{\mathrm{m} 1=0, \mathrm{n} 1=0, \mathbf{p 1}=\mathbf{0}$, $\mathbf{q} \mathbf{1}=\mathbf{0}\}$}

The divergence of the electric field intensity (electric charge density) equals:

$\nabla \cdot\left(\begin{array}{l}e_{r} \\ e_{\theta} \\ e_{\varphi}\end{array}\right)=\frac{\sqrt{2} \mathrm{~K} 1 \cot (\theta) \sin ^{2}(t \omega) \sqrt{1-\sin ^{4}(t \omega)} \mathrm{e}^{\frac{G_{1} \varepsilon_{0} \mu_{0}}{8 \pi \mathrm{r}}}}{r^{2} \sqrt{2-2 \sin ^{4}(t \omega)}}$

$\nabla .\left(\begin{array}{l}e_{r} \\ e_{\theta} \\ e_{\varphi}\end{array}\right)=\frac{\frac{1}{2} \mathrm{~K} 1 \cot (\theta) \mathrm{e}^{\frac{G_{1} \varepsilon_{0} \mu_{0}}{8 \pi \mathrm{r}}}}{r^{2}}$ (averaged over 1 period of time)

$\nabla .\left(\begin{array}{l}m_{r} \\ m_{\theta} \\ m_{\varphi}\end{array}\right)=\frac{\mathrm{K} 1 \sqrt{\varepsilon_{0}} \cot (\theta) \sqrt{\frac{3}{4}} \mathrm{e}^{\frac{G_{1} \varepsilon_{0} \mu_{0}}{8 \pi \mathrm{r}}}}{\sqrt{\mu 0} r^{2}}$ (averaged over 1 period of time)

In which $\mathrm{K} 1$ is an arbitrary variable. Because of the $\operatorname{Cot}(\theta)$ function, the electric divergence as well as the magnetic 
divergence changes from sign when the angle $\theta$ varies between $0^{\circ}$ until $360^{\circ}$ forming electric dipoles (+ versus -) and magnetic dipoles ( $\mathrm{N}$ versus $\mathrm{S}$ ). 


\subsubsection{Type 2 of "Sub Max Planck Length Gravitational- Electromagnetic Confinements" (Electric- and Magnetic Dipoles, Electric- and Magnetic Spin) $\{\mathrm{m} 1=1, \mathrm{n} 1=0, \mathrm{p} 1=0$, $\mathbf{q} \mathbf{1}=\mathbf{0}\}$}

The divergence of the electric field intensity (electric charge density) equals:

$\nabla \cdot\left(\begin{array}{l}e_{r} \\ e_{\theta} \\ e_{\varphi}\end{array}\right)=\frac{\sqrt{2} \mathrm{~K} 1 \cot (\theta) \sin ^{2}(t \omega) \sqrt{1-\sin ^{4}(t \omega)} \mathrm{e}^{\frac{G_{1} \varepsilon_{0} \mu_{0}}{8 \pi \mathrm{r}}}}{r^{2} \sqrt{2-2 \sin ^{4}(t \omega)}}$

$\nabla .\left(\begin{array}{l}e_{r} \\ e_{\theta} \\ e_{\varphi}\end{array}\right)=\frac{\frac{1}{2} \mathrm{~K} 1 \cot (\theta) \mathrm{e}^{\frac{G_{1} \varepsilon_{0} \mu_{0}}{8 \pi \mathrm{r}}}}{r^{2}}$ (averaged over 1 period of time)

The divergence of the magnetic field intensity (magnetic monopole) equals:

$\nabla .\left(\begin{array}{l}m_{r} \\ m_{\theta} \\ m_{\varphi}\end{array}\right)=\frac{\mathrm{K} 1 \sqrt{\varepsilon_{0}} \cot (\theta) \sqrt{2-2 \sin ^{4}(t \omega)} \mathrm{e}^{\frac{G_{1} \varepsilon_{0} \mu_{0}}{8 \pi \mathrm{r}}}}{\sqrt{2} \sqrt{\mu_{0}} r^{2}}$

$\nabla .\left(\begin{array}{l}m_{r} \\ m_{\theta} \\ m_{\varphi}\end{array}\right)=\frac{\mathrm{K} 1 \sqrt{\frac{3}{4}} \sqrt{\varepsilon_{0}} \cot (\theta) \mathrm{e}^{\frac{G_{1} \varepsilon_{0} \mu_{0}}{8 \pi \mathrm{r}}}}{\sqrt{\mu_{0}} r^{2}}$ (averaged over 1 period of time)

In which $\mathrm{K} 1$ is an arbitrary variable. Because of the $\operatorname{Cot}(\theta)$ function, the electric divergence as well as the magnetic divergence changes from sign when the angle $\theta$ varies between $0^{0}$ until $360^{\circ}$ forming electric dipoles ( + versus -) and magnetic dipoles ( $\mathrm{N}$ versus $\mathrm{S}$ ). 


\subsubsection{Type 3 of "Sub Max Planck Length Gravitational- Electromagnetic Confinements" $\{\mathrm{m} 1=1, \mathrm{n1}=1, \mathrm{p} 1=0$, $\mathbf{q} 1=0\}$}

The divergence of the electric field intensity (electric charge density) equals:

$\nabla .\left(\begin{array}{l}e_{r} \\ e_{\theta} \\ e_{\varphi}\end{array}\right)=\frac{\mathrm{K} 1 \sin ^{2}(t \omega) \mathrm{e}^{\frac{G_{1} \varepsilon_{0} \mu_{0}}{8 \pi \mathrm{r}}}\left(\cos (\varphi)(\sin (\theta) \sin (\varphi)+1) \sin ^{2}(t \omega)\right)}{r^{2} \sqrt{1-(\sin (\theta) \sin (\varphi)+1)^{2} \sin ^{4}(t \omega)}}+$ $\frac{\mathrm{K} 1 \sin ^{2}(t \omega) \mathrm{e}^{\frac{G_{1} \varepsilon_{0} \mu_{0}}{8 \pi \mathrm{r}}}\left((2 \cos (\theta) \sin (\varphi)+\cot (\theta)) \sqrt{1-(\sin (\theta) \sin (\varphi)+1)^{2} \sin ^{4}(t \omega)}\right)}{r^{2} \sqrt{1-(\sin (\theta) \sin (\varphi)+1)^{2} \sin ^{4}(t \omega)}}$

$\nabla .\left(\begin{array}{l}e_{r} \\ e_{\theta} \\ e_{\varphi}\end{array}\right)=\frac{\left.\mathrm{K} 1 \mathrm{e}^{\frac{G_{1} \varepsilon_{0} \mu_{0}}{8 \pi \mathrm{r}}\left(\frac{1}{2} \cos (\varphi)(\sin (\theta) \sin (\varphi)+1)\right.}\right)}{2 r^{2} \sqrt{1-\frac{1}{4}(\sin (\theta) \sin (\varphi)+1)^{2}}}+$

$\frac{\mathrm{K} 1 \mathrm{e}^{\frac{G_{1} \varepsilon_{0} \mu_{0}}{8 \pi \mathrm{r}}}\left((2 \cos (\theta) \sin (\varphi)+\cot (\theta)) \sqrt{1-\frac{1}{4}(\sin (\theta) \sin (\varphi)+1)^{2}}\right)}{2 r^{2} \sqrt{1-\frac{1}{4}(\sin (\theta) \sin (\varphi)+1)^{2}}}$ (averaged over 1 period of time) 
The divergence of the magnetic field intensity (magnetic monopole) equals:

$\nabla .\left(\begin{array}{l}m_{r} \\ m_{\theta} \\ m_{\varphi}\end{array}\right)=\frac{-\mathrm{K} 1 \sqrt{\varepsilon_{0}} \mathrm{e}^{\frac{G_{1} \varepsilon_{0} \mu_{0}}{8 \pi \mathrm{r}}}\left(\sin (\varphi) \sin ^{4}(t \omega)(\sin (2 \theta) \sin (\varphi))\right.}{\sqrt{\mu_{0}} r^{2} \sqrt{1-(\sin (\theta) \sin (\varphi)+1)^{2} \sin ^{4}(t \omega)}}+$
$\frac{\left.\mathrm{K} 1 \sqrt{\varepsilon_{0}} \mathrm{e}^{\frac{G_{1} \varepsilon_{0} \mu_{0}}{8 \pi \mathrm{r}}}(3 \cos (\theta))+\cos (\varphi) \sin ^{2}(t \omega) \sqrt{1-(\sin (\theta) \sin (\varphi)+1)^{2} \sin ^{4}(t \omega)}-\cot (\theta)\left(\sin ^{4}(t \omega)-1\right)\right)}{\sqrt{\mu_{0}} r^{2} \sqrt{1-(\sin (\theta) \sin (\varphi)+1)^{2} \sin ^{4}(t \omega)}}$

$\nabla .\left(\begin{array}{l}m_{r} \\ m_{\theta} \\ m_{\varphi}\end{array}\right)=\frac{-\mathrm{K} 1 \sqrt{\varepsilon_{0}} \mathrm{e}^{\frac{G_{1} \varepsilon_{0} \mu_{0}}{8 \pi \mathrm{r}}}\left(\sin (\varphi) \frac{1}{4}(\sin (2 \theta) \sin (\varphi)+3 \cos (\theta))\right)}{\sqrt{\mu_{0}} r^{2} \sqrt{1-\frac{1}{4}(\sin (\theta) \sin (\varphi)+1)^{2}}}+$

$\frac{\mathrm{K} 1 \sqrt{\varepsilon_{0}} \mathrm{e}^{\frac{G_{1} \varepsilon_{0} \mu_{0}}{8 \pi \mathrm{r}}}\left(\frac{1}{2} \cos (\varphi) \sqrt{1-\frac{1}{4}(\sin (\theta) \sin (\varphi)+1)^{2}}+\frac{3}{4} \cot (\theta)\right)}{\sqrt{\mu_{0}} r^{2} \sqrt{1-\frac{1}{4}(\sin (\theta) \sin (\varphi)+1)^{2}}}$ (averaged over 1 period of time)

In which $\mathrm{K} 1$ is an arbitrary variable with a Positive (positive charge) or a Negative (negative charge) value. 
3.26.4 Type 4 of "Sub Max Planck Length GravitationalElectromagnetic Confinements" (Electric- and Magnetic Dipoles, Electric- and Magnetic Spin) $\{\mathrm{m} 1=0, \mathrm{n} 1=0, \mathrm{p} 1=1$, $\mathbf{q} \mathbf{1}=\mathbf{0}\}$

The divergence of the electric field intensity (electric charge density) equals:

$\nabla \cdot\left(\begin{array}{l}e_{r} \\ e_{\theta} \\ e_{\varphi}\end{array}\right)=\frac{\mathrm{e}^{\frac{G_{1} \varepsilon_{0} \mu_{0}}{8 \pi \mathrm{r}}} K 1 \cot [\theta] \sin [t \omega]^{2}}{r^{2}}$

$\nabla .\left(\begin{array}{l}e_{r} \\ e_{\theta} \\ e_{\varphi}\end{array}\right)=\frac{\mathrm{e}^{\frac{G_{1} \varepsilon_{0} \mu_{0}}{8 \pi \mathrm{r}}} K 1 \cot [\theta]}{2 r^{2}}$ (averaged over 1 period of time) 
In which $\mathrm{K} 1$ is an arbitrary variable with a Positive (positive charge) or a Negative (negative charge) value.

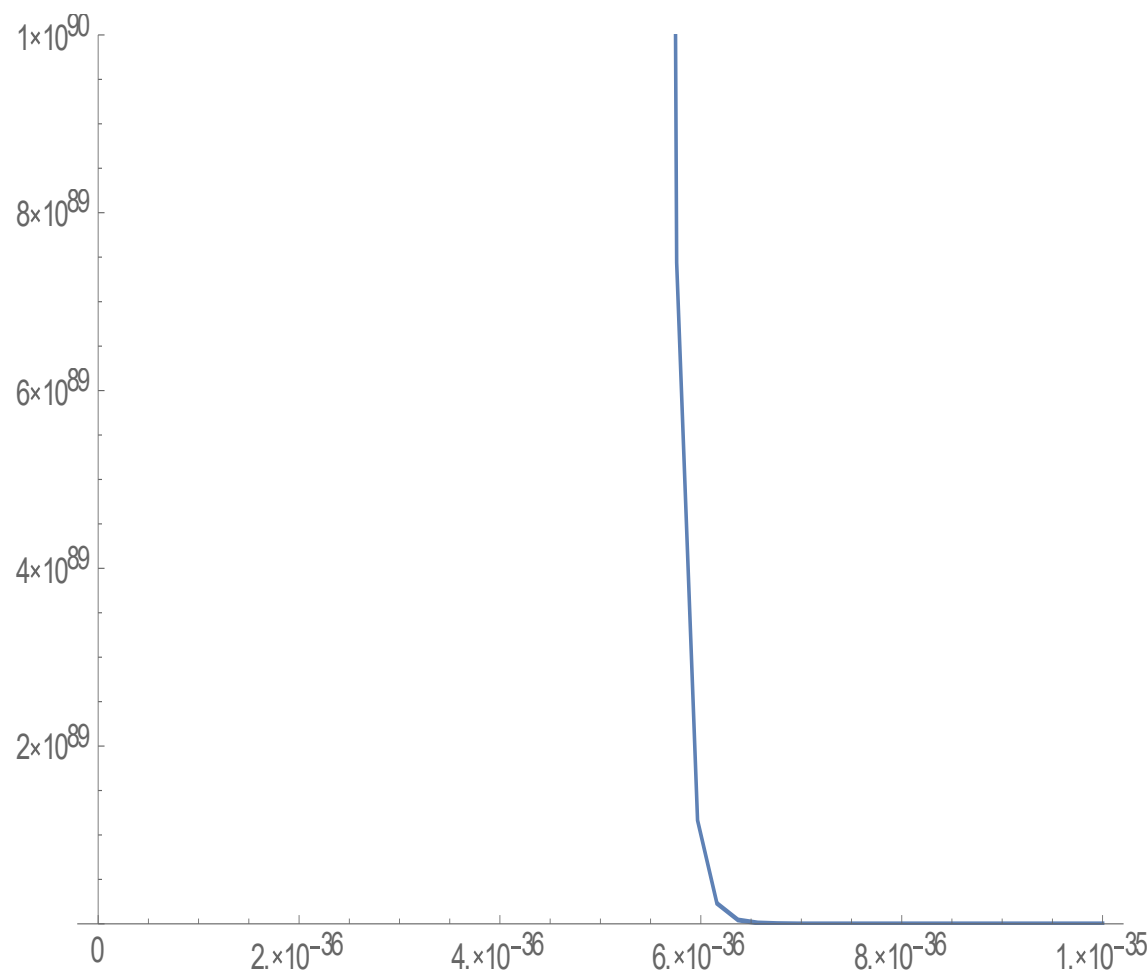

Figure 18 Equation (54). Averaged over 1 period of time. Electric Charge Density Plot in a range from $10^{-37}[\mathrm{~m}]$ until $10^{-35}[\mathrm{~m}]$. 
The divergence of the magnetic field intensity (magnetic monopole) equals:

$\nabla .\left(\begin{array}{l}m_{r} \\ m_{\theta} \\ m_{\varphi}\end{array}\right)=\frac{\mathrm{e}^{\frac{G_{1} \varepsilon_{0} \mu_{0}}{8 \pi \mathrm{r}}} K 1 \sqrt{\varepsilon_{0}} \cot [\theta] \sqrt{1-\sin [t \omega]^{4}}}{r^{2} \sqrt{\mu_{0}}}$

$\nabla .\left(\begin{array}{l}m_{r} \\ m_{\theta} \\ m_{\varphi}\end{array}\right)=\frac{\mathrm{e}^{\frac{G_{1} \varepsilon_{0} \mu_{0}}{8 \pi \mathrm{r}}} K 1 \sqrt{\varepsilon_{0}} \cot [\theta]}{2 r^{2} \sqrt{\mu_{0}}}$ (averaged over 1 period of time)

In which $\mathrm{K} 1$ is an arbitrary variable. Because of the $\operatorname{Cot}(\theta)$ function, the electric divergence as well as the magnetic divergence changes from sign when the angle $\theta$ varies between $0^{\circ}$ until $360^{\circ}$ forming electric dipoles (+ versus -) and magnetic dipoles ( $\mathrm{N}$ versus $\mathrm{S}$ ). 


\subsubsection{Type 5 of "Sub Max Planck Length Gravitational- Electromagnetic Confinements" $\{\mathrm{m} 1=0, \mathrm{n1}=\mathbf{0}, \mathbf{p 1}=1$, $\mathrm{q} 1=\mathbf{1}\}$}

The divergence of the electric field intensity (electric charge density) equals:

$\nabla .\left(\begin{array}{l}e_{r} \\ e_{\theta} \\ e_{\varphi}\end{array}\right)=\frac{\mathrm{e}^{\frac{G_{1} \varepsilon_{0} \mu_{0}}{8 \pi \mathrm{r}}} K 1\left(2 \cot [\theta] \sin [t \omega]^{2}-\frac{\cos [\varphi]}{\sqrt{1+\sin [\theta] \sin [\varphi]-\sin [t \omega]^{4}}}\right)}{2 r^{2}}$

$\nabla .\left(\begin{array}{l}e_{r} \\ e_{\theta} \\ e_{\varphi}\end{array}\right)=\frac{\mathrm{e}^{\frac{G_{1} \varepsilon_{0} \mu_{0}}{8 \pi \mathrm{r}}} K 1\left(\cot [\theta]-\frac{\cos [\varphi]}{\sqrt{\frac{3}{4}+\sin [\theta] \sin [\varphi]}}\right)}{2 r^{2}} \quad$ (averaged over 1 period of time)

The divergence of the magnetic field intensity (magnetic monopole) equals:

$\nabla .\left(\begin{array}{l}m_{r} \\ m_{\theta} \\ m_{\varphi}\end{array}\right)=\frac{\mathrm{e}^{\frac{G_{1} \varepsilon_{0} \mu_{0}}{8 \pi \mathrm{r}}} K 1 \sqrt{\varepsilon_{0}}\left(3 \cos [\theta] \sin [\varphi]-2 \cot [\theta]\left(-1+\sin [t \omega]^{4}\right)\right)}{2 r^{2} \sqrt{\mu_{0}} \sqrt{1+\sin [\theta] \sin [\varphi]-\sin [t \omega]^{4}}}$

$\nabla .\left(\begin{array}{l}m_{r} \\ m_{\theta} \\ m_{\varphi}\end{array}\right)=\frac{\mathrm{e}^{\frac{G_{1} \varepsilon_{0} \mu_{0}}{8 \pi \mathrm{r}}} K 1 \sqrt{\varepsilon_{0}}\left(3 \cos [\theta] \sin [\varphi]+\frac{3}{2} \cot [\theta]\right)}{2 r^{2} \sqrt{\mu_{0}} \sqrt{\frac{3}{4}+\sin [\theta] \sin [\varphi]}}$ (averaged over 1 period of time)

In which $\mathrm{K} 1$ is an arbitrary variable with a Positive (positive charge) or a Negative (negative charge) value. 


\subsection{Type II of "Sub Max Planck Length Gravitational- Electromagnetic Confinements"}

The "sub Max Planck's length" Type II confinement has been described for the electric field intensity:

$$
\left(\begin{array}{l}
e_{r} \\
e_{\theta} \\
e_{\varphi}
\end{array}\right)=\left(\begin{array}{c}
0 \\
\frac{\mathrm{e}^{\frac{G 1 \varepsilon_{0} \mu_{0}}{8 \pi \mathrm{r}}} h[\theta, \varphi] \sin [\omega t]^{2} \sin \left[r \sqrt{\varepsilon_{0} \mu_{0}} \omega\right]^{2}}{r} \\
-\frac{\mathrm{e}^{\frac{G 1 \varepsilon_{0} \mu_{0}}{8 \pi \mathrm{r}}} h[\theta, \varphi] \sqrt{K 1-\sin [\omega t]^{4} \sin \left[r \sqrt{\varepsilon_{0} \mu_{0}} \omega\right]^{4}}}{r}
\end{array}\right)
$$

The "sub Max Planck's length" confinement has been described for the magnetic field intensity:

$$
\left(\begin{array}{l}
m_{r} \\
m_{\theta} \\
m_{\varphi}
\end{array}\right)=\sqrt{\frac{\varepsilon_{0}}{\mu_{0}}}\left(\begin{array}{c}
0 \\
\frac{\mathrm{e}^{\frac{G 1 \varepsilon_{0} \mu_{0}}{8 \pi \mathrm{r}}} h[\theta, \varphi] \sqrt{K 1-\sin [\omega t]^{4} \sin \left[r \sqrt{\varepsilon_{0} \mu_{0}} \omega\right]^{4}}}{r} \\
\frac{\mathrm{e}^{\frac{G 1 \varepsilon_{0} \mu_{0}}{8 \pi \mathrm{r}}} h[\theta, \varphi] \sin [\omega t]^{2} \sin \left[r \sqrt{\varepsilon_{0} \mu_{0}} \omega\right]^{2}}{r}
\end{array}\right)
$$


The divergence of the electric field intensity (electric charge density) equals:

$\begin{aligned} \nabla .\left(\begin{array}{l}e_{r} \\ e_{\theta} \\ e_{\varphi}\end{array}\right)= & \frac{\mathrm{e}^{\frac{G 1 \epsilon 0 \mu 0}{8 \pi r}} \sqrt{K 1-\sin [t \omega]^{4} \sin \left[r \sqrt{\varepsilon_{0} \mu_{0}} \omega\right]^{4}} h^{(0,1)}[\theta, \varphi]}{r} \\ & +\frac{\mathrm{e}^{\frac{G 1 \epsilon 0 \mu 0}{8 \pi r}} \sin [t \omega]^{2} \sin \left[r \sqrt{\varepsilon_{0} \mu_{0}} \omega\right]^{2} h^{(1,0)}[\theta, \varphi]}{r}\end{aligned}$

In which $\mathrm{K} 1$ is a positive constant equal or larger than 1.

The divergence of the magnetic field intensity (magnetic monopole) equals:

$\begin{aligned} \nabla .\left(\begin{array}{c}m_{r} \\ m_{\theta} \\ m_{\varphi}\end{array}\right)= & \frac{\mathrm{e}^{\frac{G_{1} \varepsilon_{0} \mu_{0}}{8 \pi \mathrm{r}}} \sqrt{\varepsilon_{0}} \sin [t \omega]^{2} \sin \left[r \sqrt{\varepsilon_{0} \mu_{0}} \omega\right]^{2} h^{(0,1)}[\theta, \varphi]}{r \sqrt{\mu_{0}}} \\ & +\frac{\mathrm{e}^{\frac{G_{1} \varepsilon_{0} \mu_{0}}{8 \pi \mathrm{r}}} \sqrt{\varepsilon_{0}} \sqrt{K 1-\sin [t \omega]^{4} \sin \left[r \sqrt{\varepsilon_{0} \mu_{0}} \omega\right]^{4}} h^{(1,0)}[\theta, \varphi]}{r \sqrt{\mu_{0}}}\end{aligned}$

The function has been chosen:

$h[\theta, \varphi]=\sin [n \theta] \cos [m \varphi]$

In which the integers $\mathrm{n}=0,1 / 2,1 \frac{1}{2}, 2,2 \frac{1}{2}, 3,3 \frac{1}{2}, \ldots$. And $\mathrm{m}$ $=0,1 \frac{1}{2}, 1 \frac{1}{2}, 2,2 \frac{1}{2}, 3,31 \frac{1}{2}, \ldots$.

$\rho=\varepsilon_{0} \nabla \cdot\left(\begin{array}{l}e_{r} \\ e_{\theta} \\ e_{\varphi}\end{array}\right)=\frac{n \varepsilon_{0} \mathrm{e}^{\frac{G l \epsilon \mu \mu 0}{8 \pi r}} \cos (n \theta) \cos (m \varphi) \sin (t \omega)^{2} \sin \left(r \sqrt{\varepsilon_{0} \mu_{0}} \omega\right)^{2}}{r}$

According to Gauss's law the electric charge density $\rho$ equals for $\mathrm{m}=0$ and $\mathrm{n}=1$ for an electric monopole. 239 
Equation (63) represents a di-pole function for the electric charge density.

For the corresponding magnetic di-pole flux density $\phi$ (spin) equals for $n=0$ and $m=+1 / 2($ spin up) and $m=-1 / 2($ spin down):

$\phi=\mu_{0} \nabla .\left(\begin{array}{c}m_{r} \\ m_{\theta} \\ m_{\varphi}\end{array}\right)=\frac{m \sqrt{\varepsilon_{0} \mu_{0}} \mathrm{e}^{\frac{G l<\theta_{0} 0}{8 \pi r}} \cos (n \theta) \cos (m \varphi) \sin \left(r \sqrt{\varepsilon_{0} \mu_{0}} \omega\right)^{2}}{r}$

Equation (64) represents a di-pole function for the magnetic flux density (spin). 


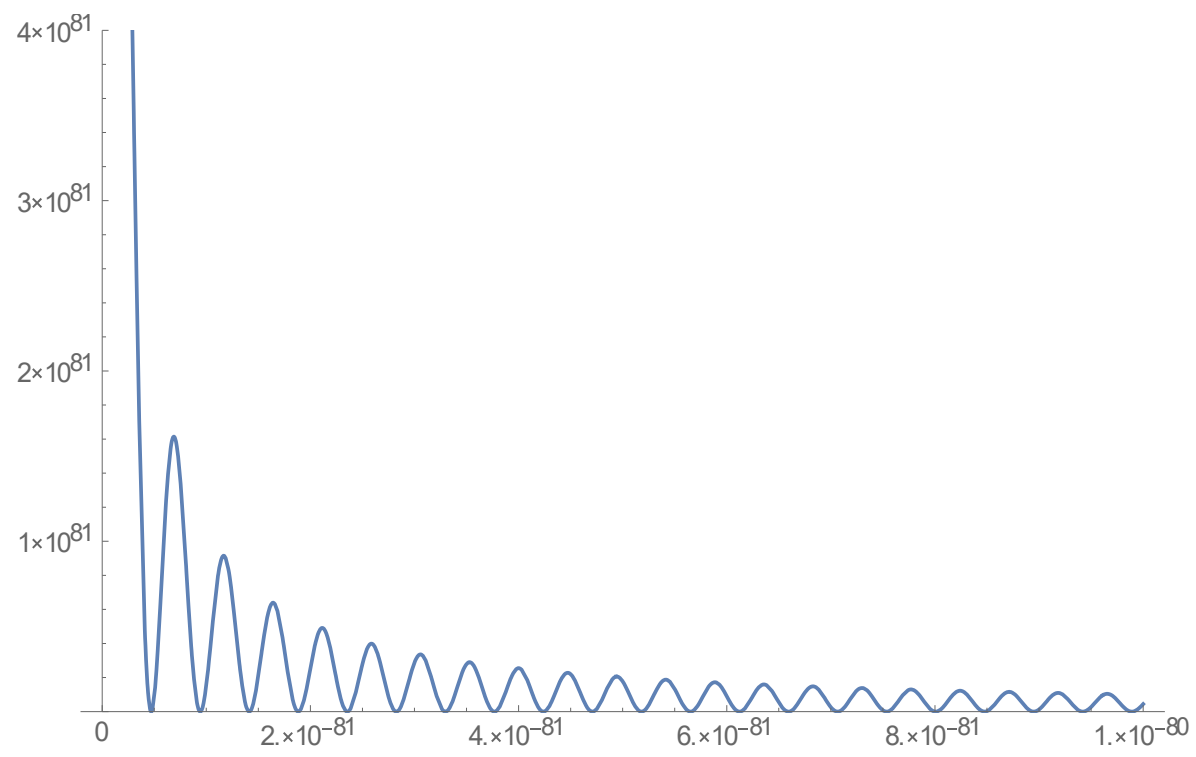

Figure 19 Equation (56): PlotGraph of the Electric Field Intensity $\mathrm{f}(\mathrm{r})$ for the region $10^{-85}<\mathrm{r}<\mathbf{1 0}^{-80}$ with a frequency of $\omega=10^{90}\left[\mathrm{~s}^{-1}\right]$ in which the gravitational field acceleration has been chosen accordingly an electromagnetic mass of $1.6726 \times 10^{-27}[\mathrm{~kg}]$ located at the center of the confinement, according Newton's Shell Theorem. 


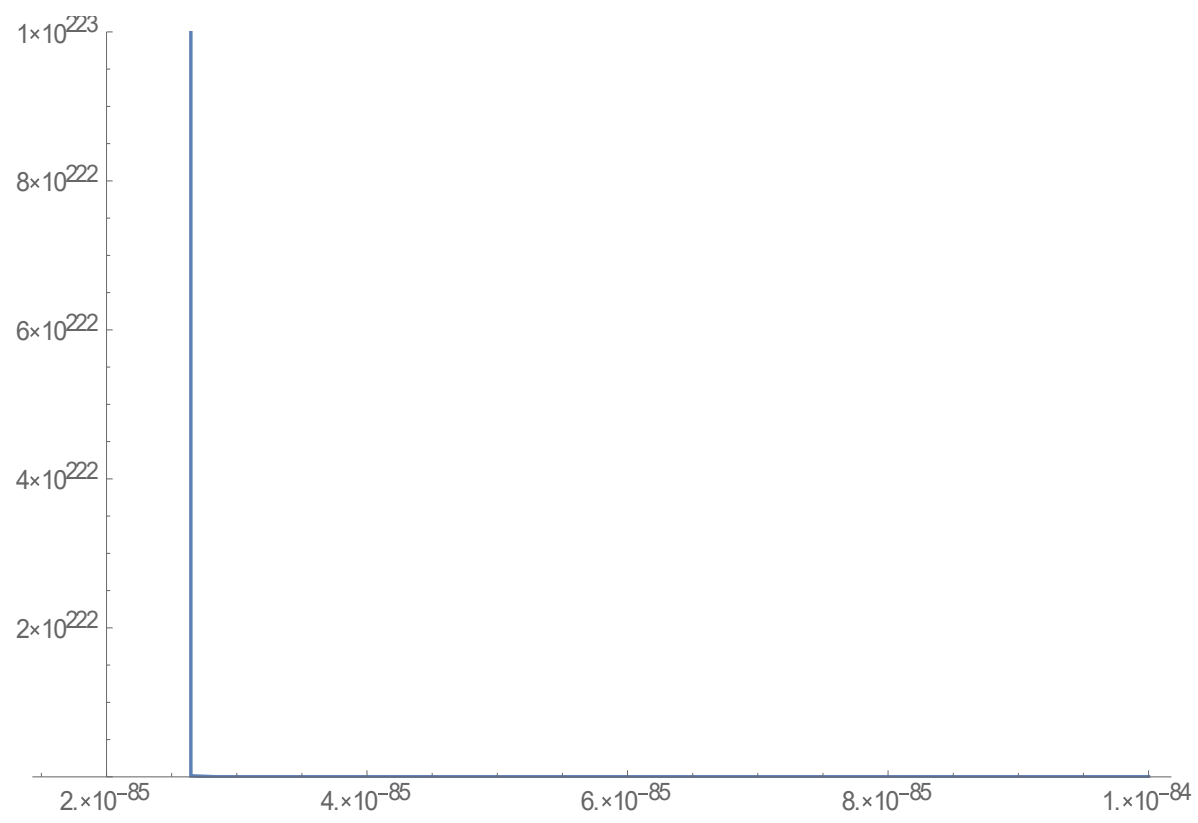

Figure 20 Equation (56): PlotGraph of the Electric Field Intensity $\mathrm{f}(\mathrm{r})$ for the region $10^{-85}<\mathrm{r}<\mathbf{1 0}^{-84}$ with a frequency of $\omega=10^{90}$ in which the gravitational field acceleration has been chosen accordingly an electromagnetic mass of $1.6726 \times 10^{-27}[\mathrm{~kg}]$ located at the center of the confinement, according Newton's Shell Theorem. 


\subsection{Concluding Remarks}

The example of Gravitational-Electromagnetic Interaction, presented in table 1 shows two types of confinement.

1. For values $0<\mathrm{n}<-1$, the GravitationalElectromagnetic Confinement will be Gravitationally controlled (Table 1). This means that for values for $r>R_{\text {BOUNDARY }}$ the inward bounded Gravitational for will be larger than the outward bounded Electromagnetic Radiation pressure. Electromagnetic Radiation will be attracted by Gravity towards the confinement at the surface $R_{\text {BOUNDARY }}$. Because for values $r<R_{\text {BOUNDARY }}$ the outward bounded radiation pressure is higher than the inward bounded gravitational pressure, all the radiation will be forced to be confined at equilibrium just at the surface of the spherical sphere with radius $\mathrm{R}_{\text {BOUNDARY }}$. The confinement can be considered as an Electromagnetic Black Hole.

2. For values $-1<\mathrm{n}<-\infty$, the GravitationalElectromagnetic Confinement will be Electromagnetically controlled (Table 1). This means that for values for $r>R_{\text {BOUNDARY }}$ the inward bounded Gravitational for will be smaller than the outward bounded Electromagnetic Radiation pressure. Electromagnetic Radiation will be scattered by the Radiation Pressure away from the confinement at the surface $R_{\text {BOUNDARY }}$. Because for values $r<R_{\text {BOUNDARY }}$ the outward bounded radiation pressure is smaller than 
the inward bounded gravitational pressure, all the radiation will be confined within the sphere with radius $\mathrm{R}_{\text {BOUNDARY }}$. The confinement can be considered as an Electromagnetic Particle.

3. For values $n=-1$, the inward bounded Gravitational pressure equals the outward bounded Electromagnetic Radiation pressure at any distance $\mathrm{r}$. The calculated value for $\mathrm{R}_{\text {BOUNDARY }}$ becomes $\mathrm{R}_{\text {BOUNDARY }} \rightarrow \infty$. Because of the extremely high-energy densities within electromagnetic-gravitational confinements and the extremely small dimensions, the radiation pressure at small densities will be extremely high. For this reason, single harmonic (monochromatic) electromagnetic-gravitational confinements will behave like nondeformable particles in experiments.

Table 1 demonstrates that the mass density division within the Electromagnetic Gravitational Confinement determines a wide range of diameters of the Confinements varying from $10^{-52}[\mathrm{~m}]$ (which is $10^{-42}$ times smaller than the diameter of the atom) until $10^{54}[\mathrm{~m}]$ (which is $10^{27}$ times larger than the diameter of the observable universe).

The exact solution for a Gravitational Electromagnetic Confinement results in diameters much smaller than Planck's Length (1.616229 $\left.10^{-35}[\mathrm{~m}]\right)$.

The fundamental question is: How it is possible to create confinements from "visible light" (with a wave length between $3.9 \times 10^{-7}[\mathrm{~m}]$ until $\left.7 \times 10^{-7}[\mathrm{~m}]\right)$ within dimensions smaller than Planck's Length? 
This is only possible when the wave length of the confined radiation is smaller than de dimensions of the confinement. This requires extreme high frequencies. The transformation in frequency from visible light into the extreme high frequency of the confinement is possible because of the Lorentz transformation during the collapse of the radiation when the confinement has been formed (implosion of visible light). 


\subsection{Three fundamental Equations in Qantum Physics}

Taking the 4-dimensional Divergence of the 4-dimensional Stress Energy Tensor ${ }^{(1,16,29)}$ (Equation 3 ) results in a 4dimensional 4-vector with 4 components, which can be presented as 3 fundamentally different Electromagnetic/ Quantum Mechanical equations.

1) The $4^{\text {th }}$ component of this 4 -vector equals Poynting's Theorem. Evidence has been presented that the Schrödinger Wave Equation is a complex notation for Poynting's Theorem (equation 42, Ref. 29) rewritten in a complex way (equation 55, Ref.29)

2) The $4^{\text {th }}$ component of this 4 -vector equals Poynting's Theorem. Evidence has been presented that the relativistic Dirac Equation is a complex notation for Poynting's Theorem (equation 57, Ref. 29) rewritten in a complex way (equation 102, Ref.29)

3) The first 3 components of this 4-vector result in the 3-Dimensional Vector Equations (5) and (5-a) describing the "Unified 4-Dimensional Hyperspace Equilibrium" (beyond Einstein 4-Dimensional, Kaluza-Klein 5-Dimensional and Superstring 10- and 11 Dimensional Curved Hyperspaces). The fourth component of this 4-vector results in the relativistic Dirac Equation (5.P). 


\subsection{Acknowledgment}

I specially would like to thank Professor Dr. F.W. Sluijter (1936-2014) TU/e, The Netherlands. He has shown a way in physics that has been forgotten by so many. A humble man man with a great wisdom. He has always been one of the happy few of the superior level of scientists like Albert Einstein who think completely independently. Albert Einstein, whose ideas have been the foundation of this work. A man of wisdom, independently thinking and a great insight in Physics. He had the courage and vision to create a new unknown path in physics to find beyond our understanding that what unifies the Universe.

I am indebted to all who have contributed to the modern science we have achieved after many centuries of sacrifice by so many unknown scientists. And specially I am indebted to my family, who always have always supported me on a long and winding road in life and in physics.

\subsection{Data Availability}

All the Data and all the Calculations to provide evidence to this 'New Theory about Light' have been published in the 'Open Source Framework(OSF)': https://osf.io/gbn4p/

DOI: 10.31219/osf.io/gbn4p ( https://doi.org/10.31219/osf.io/gbn4p)

(Calculations in Mathematica 11.0)', Page 1-33). 


\section{References}

1. O. Gunawan, Y. Virgus, and K. Fai Tai, A parallel dipole line system, Appl. Phys. Lett. 106, 062407 (2015).

2. O. Gunawan and Y. Virgus, The one-dimensional camelback potential in the parallel dipole line trap: Stability conditions and finite size effect, J. Appl. Phys. 121, 133902 (2017).

3. O. Gunawan and Q. Cao, "Magnetic trap for cylindrical diamagnetic materials," U.S. patent 8,895,355 (2014); 9,093,377 (2015); 9,236,293 (2016); 9,263,669 (2016); 9,424,971 (2016).

4. Dirk Englund, Arka Majumdar, Michal Bajcsy, Andrei Faraon, Pierre Petroff, and Jelena Vučković; Ultrafast Photon-Photon Interaction in a Strongly Coupled Quantum Dot-Cavity System, Phys. Rev Lett. 108, 093604, March 2012, DOI : 10.1103/PhysRevLett.108.093604

5. Z. K. Minev, S. O. Mundhada, S. Shankar, P. Reinhold, R. Gutiérrez-Jáuregui, R.J. Schoelkopf, N. Mirrahimi, H.J. Carmichael and M. H. Devoret; To catch and reverse a quantum jump mid-flight; Nature; 03 June 2019

6. Ryotaro Kase, Masato Minamitsuji, and Shinji Tsujikawa; Relativistic stars in vector-tensor theories ; Phys. Rev. D 97, 084009 - Published 9 April 2018

7. Hector O. Silva, Jeremy Sakstein, Leonardo Gualtieri, Thomas P. Sotiriou, and Emanuele Berti;

Spontaneous Scalarization of Black Holes and Compact Stars from a Gauss-Bonnet Coupling;Phys. Rev. Lett. 120, 131104 (2018) - Published 30 March 2018 
8. Jahed Abedi, Hannah Dykaar, and Niayesh Afshordi; Echoes from the abyss: Tentative evidence for Planckscale structure at black hole horizons ;Phys. Rev. D 96, 082004 (2017) - Published 26 October 2017

9. A. Hees, T. Do, A. M. Ghez, G. D. Martinez, S. Naoz, E. E. Becklin, A. Boehle, S. Chappell, D. Chu, A. Dehghanfar, K. Kosmo, J. R. Lu, K. Matthews, M. R. Morris, S. Sakai, R. Schödel, and G. Witzel; Testing General Relativity with Stellar Orbits around the Supermassive Black Hole in Our Galactic Center; Phys. Rev. Lett. 118, 211101 (2017) - Published 25 May 2017

10. Petr Hořava; Spectral Dimension of the Universe in Quantum Gravity at a Lifshitz Point; Phys. Rev. Lett. 102, 161301 (2009); Published April 20, 2009

11. Kenji Hayashi and Takeshi Shirafuji ; Addendum to "New general relativity" ; Phys. Rev. D 24, 3312 Published 15 December 1981

12 Talmadge M. Davis and John R. Ray ; Ghost neutrinos in general relativity; Phys. Rev. D 9, 331 (1974) - Published 15 January 1974

13 Patrick G. Whitman and Richard C. Burch; Charged spheres in general relativity; Phys. Rev. D 24, 2049 Published 15 October 1981; Erratum Phys. Rev. D 25, 1744 (1982)

14 Joseph Jacobson, Gunnar Björk, Isaac Chuang, and Yoshihisa Yamamoto ; Photonic de Broglie Waves ; Phys. Rev. Lett. 74, 4835 (1995) - Published 12 June 1995

15 Bogeun Gwak and Bum-Hoon Lee ; Instability of rotating anti-de Sitter black holes; Phys. Rev. D 91, 064020 - Published 9 March 2015

16 Raphael Bousso and Stephen Hawking ; Erratum: Lorentzian condition in quantum gravity [Phys. Rev. D 59, 103501 (1999)] ; Phys. Rev. D 60, 109903 (1999) - Published 8 October 1999 
17 A. Steane, P. Szriftgiser, P. Desbiolles, and J. Dalibard; Phase Modulation of Atomic de Broglie Waves ; Phys. Rev. Lett. 74, 4972 - Published 19 June 1995

18 S. B. Cahn, A. Kumarakrishnan, U. Shim, T. Sleator, P. R. Berman, and B. Dubetsky; Time-Domain de Broglie Wave Interferometry ; Phys. Rev. Lett. 79, 784 - Published 4 August 1997

19 J. L. Synge; Primitive Quantization in the Relativistic Two-Body Problem ; Phys. Rev. 89, 467 - Published 15 January 1953

20 H. Jehle, Flux Quantization and particle Physics, Phys. Rev. D6 (1972) 441 - 457

21 Osung Kwon, Young-Sik Ra, and Yoon-Ho Kim ; Observing photonic de Broglie waves without the maximally-path-entangled $|\mathrm{N}, 0\rangle+|0, \mathrm{~N}\rangle$ state ; Phys. Rev. A 81, 063801 (2010) - Published 1 June 2010

22 V. Krachmalnicoff, J.-C. Jaskula, M. Bonneau, V. Leung, G. B. Partridge, D. Boiron, C. I. Westbrook, P. Deuar, P. Ziń, M. Trippenbach, and K. V. Kheruntsyan ; Spontaneous Four-Wave Mixing of de Broglie Waves: Beyond Optics ; Phys. Rev. Lett. 104, 150402 (2010) - Published 15 April 2010

23 Andrey Turlapov, Alexei Tonyushkin, and Tycho Sleator ; Talbot-Lau effect for atomic de Broglie waves manipulated with light; Phys. Rev. A 71, 043612 (2005) - Published 25 April 2005

24 Jakob Petersen, Eli Pollak, and Salvador Miret-Artes ; Alberto Nicolis and Riccardo Penco ; Mutual interactions of phonons, rotons, and gravity ; Phys. Rev. B 97, 134516 (2018) - Published 18 April 2018

25 Quantum threshold reflection is not a consequence of a region of the long-range attractive potential with 
rapidly varying de Broglie wavelength; Phys. Rev. A 97, 042102 (2018) - Published 3 April 2018

26 Jing, H., Jiang, Y. \& Deng, Y.; Quantum superchemistry of de Broglie waves: New wonderland at ultracold temperature ; Front. Phys. China (2011) 6: 15. https://doi.org/10.1007/s11467010-0155-y

27 Donald H Kobe ; Quantum power in de BroglieBohm theory ; Journal of Physics A: Mathematical and Theoretical, Volume 40 - Number 19 , Published 24 April 2007

28 Rodewald, J., Haslinger, P., Dörre, N. et al.; New avenues for matter-wave-enhanced spectroscopy ; Appl. Phys. B (2017) 123: 3.

https://doi.org/10.1007/s00340-016-6573-y

29. J. W. Vegt, A Continuous Model of Matter based on AEONs, Physics Essays ,1995, Volume 8, Number 2, 201-224 A Continuous model of Matter (DOI: 10.13140/RG.2.2.25149.77281).

30. J. W. Vegt, Annales Fondation Louis de Broglie, The Maxwell-Schrödinger-Dirac Correspondence in Auto Confined Electromagnetic Fields, Annales Fondation Louis de Broglie, 2002, Volume 27, Number 1,

31. J. W. Vegt, A particle Free Model of Matter based on Electromagnetic Self-Cofinement, Annales Fondation Louis de Broglie, 1996, January.

32. J. M. Maldacena, Black Holes in String Theory, Princeton University, arxiv.org/abs/hepth/960723533.

33. V. C. de Andrade and J. G. Pereira, Gravitational Lorentz force and the description of the gravitational interaction, Phys. Rev. D 56, 468

34. Mohr, P.J.; Taylor, B.N.; Newell, D.B. (2006). "CODATA recommended values of the fundamental physical constants". Reviews of Modern Physics. 80(2): 633-730. arXiv:0801.0028, 
Bibcode:2008RvMP...80..633M. doi:10.1103/RevMo dPhys.80.633.

35. J. M. Maldacena, Black Holes in String Theory, Princeton University, arxiv.org/abs/hepth/960723533.

36. V. C. de Andrade and J. G. Pereira, Gravitational Lorentz force and the description of the gravitational interaction, Phys. Rev. D 56, 468

37. Mohr, P.J.; Taylor, B.N.; Newell, D.B. (2006). "CODATA recommended values of the fundamental physical constants". Reviews of Modern Physics. 80(2): 633-730. arXiv:0801.0028, Bibcode:2008RvMP...80..633M. doi:10.1103/RevMo dPhys.80.633.

38. William A. Hiscock, Phys. Rev. D 31, 3288 Published 15 June 1985

39. T. Degrand, L. Jaffe, K Johnson, J. Kiskis, Masses and other parameters of the light Hadrons, Physical Review D: Particles and Fields, 12(7), October 1975, 2060-2076

40 Volodymyr Krasnoholovets, Motion of a Relativistic Particle and the Vacuum, Physics Essays, vol 10, no 3, 1997, 407-416, arXiv:quant-ph/9903077

41 H. Jehle, Flux Quantization and fractional charges of quarks, Phys. Rev. D11(1975) 2147

42 W.G.V. Rosser, Classical Electromagnetism via Relativity (Butterworths, London, 1968), p. 134.

43. Brando Bellazzini, Francesco Riva, Javi Serra, and Francesco Sgarlata; Beyond Positivity Bounds and the Fate of Massive Gravity ; Phys. Rev. Lett. 120, 161101 - Published 17 April 2018

44. Bao-Fei Li, Parampreet Singh, and Anzhong Wang; Towards cosmological dynamics from loop quantum gravity ;Phys. Rev. D 97, 084029 - Published 17 April 2018 
45. Li-Xin Li; A New Unified Theory of Electromagnetic and Gravitational Interactions, Frontiers of Physics, Volume 11, Issue 6, article id. 110402 (2016); arxiv.org/abs/1511.01260

46. Richard Easther, Brian R Greene, Mark G Jackson and Daniel Kabat; String windings in the early universe, Journal of Cosmology and Astroparticle Physics, Volume 2005, February 2005

47. J. Wheeler, Phys. Rev. 97, 511 (1955).

48. L. Filipe O. Costa, Georgios Lukes-Gerakopoulos, and Oldřich Semerák; Spinning particles in general relativity: Momentum-velocity relation for the Mathisson-Pirani spin condition; Phys. Rev. D 97, 084023 - Published 16 April 2018 